University of Louisville

ThinkIR: The University of Louisville's Institutional Repository

Electronic Theses and Dissertations

$12-2014$

\title{
Study of novel nanoparticle transport and drug release for cancer treatment.
}

Christopher Gene England 1988-

University of Louisville

Follow this and additional works at: https://ir.library.louisville.edu/etd

Part of the Pharmacy and Pharmaceutical Sciences Commons

\section{Recommended Citation}

England, Christopher Gene 1988-, "Study of novel nanoparticle transport and drug release for cancer treatment." (2014). Electronic Theses and Dissertations. Paper 1712.

https://doi.org/10.18297/etd/1712

This Doctoral Dissertation is brought to you for free and open access by ThinkIR: The University of Louisville's Institutional Repository. It has been accepted for inclusion in Electronic Theses and Dissertations by an authorized administrator of ThinkIR: The University of Louisville's Institutional Repository. This title appears here courtesy of the author, who has retained all other copyrights. For more information, please contact thinkir@louisville.edu. 
STUDY OF NOVEL NANOPARTICLE TRANSPORT AND DRUG RELEASE FOR CANCER TREATMENT

\title{
By
}

Christopher England

B.S.; Bellarmine University, 2011

M.S.; University of Louisville, 2013

\author{
A Dissertation \\ Submitted to the Faculty of the \\ School of Medicine of the University of Louisville \\ In Partial Fulfillment of the Requirements \\ For the Degree of \\ Doctor of Philosophy \\ Department of Pharmacology and Toxicology \\ University of Louisville \\ Louisville, Kentucky
}

December 2014 

STUDY OF NOVEL NANOPARTICLE TRANSPORT AND DRUG RELEASE FOR CANCER TREATMENT

\section{By}

Christopher England

B.S; Bellarmine University, 2011

M.S., University of Louisville, 2013

A Dissertation Approved on

November 13, 2014

By the following Dissertation Committee:

Hermann Frieboes (Chair)

Brian Ceresa

Jill Steinbach

Martin O'Toole

Albert Cunningham 


\section{ACKNOWLEDGEMENTS}

I would like to thank my advisor, Dr. Hermann Frieboes, for his guidance throughout my graduate studies. I would also like to thank the other committee members for their valuable assistance, including Dr. Brian Ceresa, Dr. Martin O’Toole, Dr. Jill Steinbach, and Dr. Albert Cunningham. Additionally, my gratitude is extended to all collaborators at the University of Louisville, without whom the work for this thesis could not have been accomplished. 


\title{
ABSTRACT \\ STUDY OF NOVEL NANOPARTICLE TRANSPORT AND DRUG RELEASE FOR CANCER TREATMENT
}

\author{
Christopher G. England
}

November 13, 2014

Nano-scale particles sized $10-400 \mathrm{~nm}$ administered systemically preferentially extravasate from tumor vasculature due to the enhanced permeability and retention effect. Therapeutic success remains elusive, however, because of inhomogeneous particle distribution within tumor tissue. Insufficient tumor vascularization limits particle transport and also results in avascular hypoxic regions with non-proliferating cells, which can regenerate tissue after nanoparticle-delivered cytotoxicity or thermal ablation. In this study, gold nanoparticles were functionalized with phosphatidylcholine (two-layer) or phosphatidylcholine and HDL (three-layer) in the formation of "layered" nanoparticles. The diffusivity of both two- and three layered colloidal gold nanoparticles and silica gold nanoshells were examined in 3D cell cultures. Both two- and three layered nanoparticles showed enhanced diffusivity in comparison to previously developed PEGylated nanoparticles. As the two layer nanoparticles displayed enhanced diffusivity in comparison to three layer nanoparticles, the two layered nanoparticles were further examined in vivo using mice implanted with orthotopic pancreatic adenocarcinomas. The two layer colloidal gold nanoparticles showed enhanced diffusivity in comparison to silica gold nanoshells in vivo, suggesting that smaller nanoparticles were able to localize and diffuse from vasculature better than larger nanoparticles. Overall accumulation of solid gold nanoparticle accumulated in the tumor and filtering organs (liver and spleen) was $2 \mathrm{X}$ higher than silica gold nanoshells. Thus, two layer colloidal gold nanoparticles were loaded with cisplatin or paclitaxel to determine optimal drug release kinetics. Drug release from paclitaxel-loaded nanoparticles displayed a slower release while cisplatin-loaded nanoparticles experienced an initial burst of drug release followed by a slower release of remaining drug. Lastly, drug-loaded colloidal gold nanoparticles were tested in 3D cell cultures to determine their cytotoxicity. Both two and three layer nanoparticles loaded with cisplatin or 
paclitaxel showed similar efficacy to drug alone, suggesting their viable use in vivo for cancer treatment. This study has demonstrated the potential use of layered nanoparticles for increasing the delivery of chemotherapeutics deeper into tumor tissue. 
TABLE OF CONTENTS

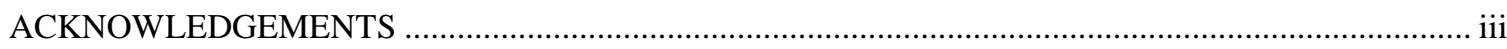

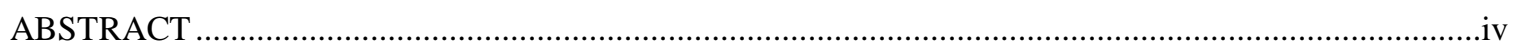

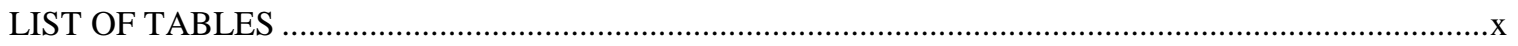

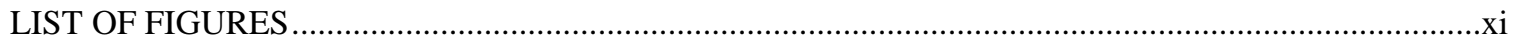

INTRODUCTION TO NANOTHERAPEUTICS IN CANCER TREATMENT ……..................................

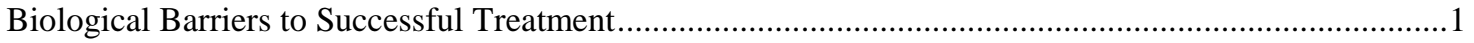

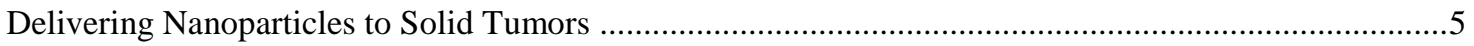

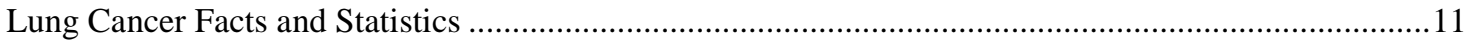

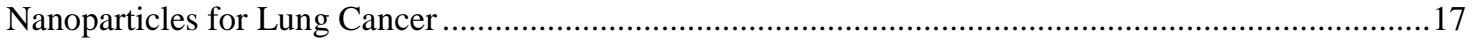

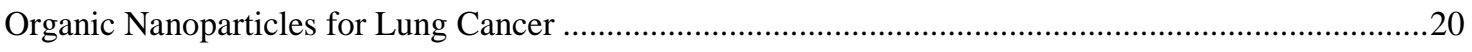

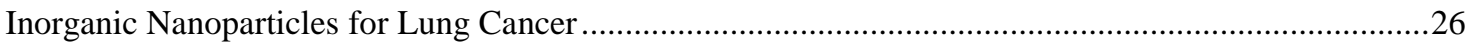

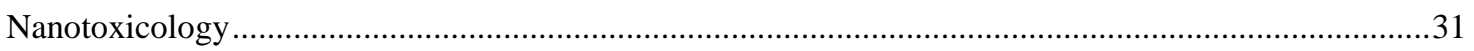

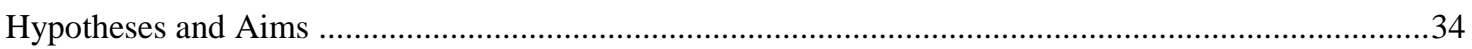

ENHANCED PENETRATION INTO 3D CELL CULTURES USING TWO- AND THREE LAYERED

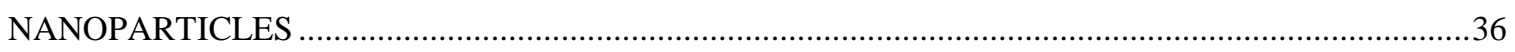

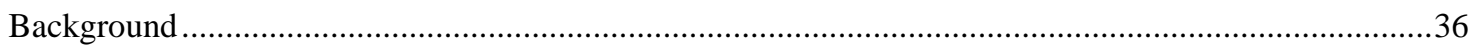

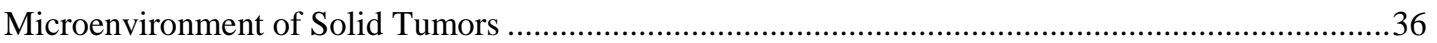

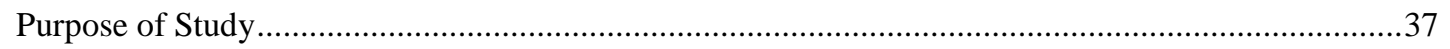

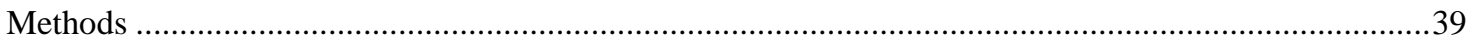

Synthesis of Citrate Gold Nanoparticles.............................................................................................

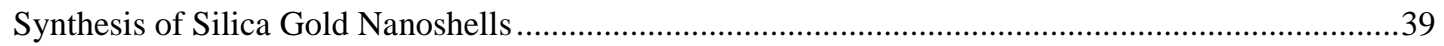

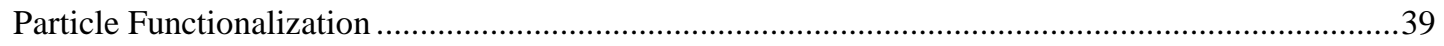

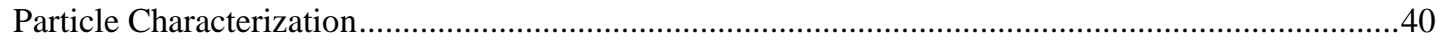

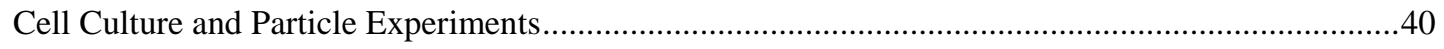

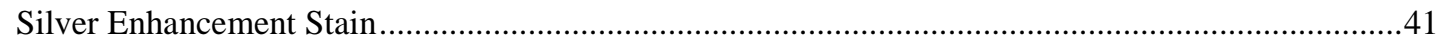

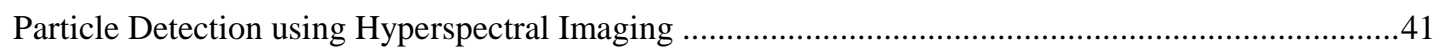

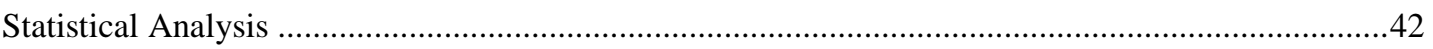


Results

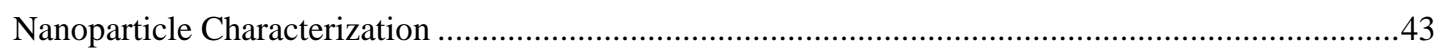

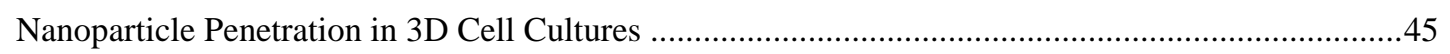

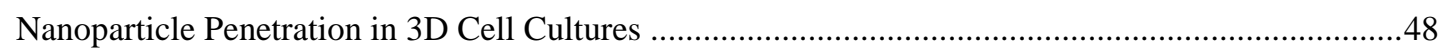

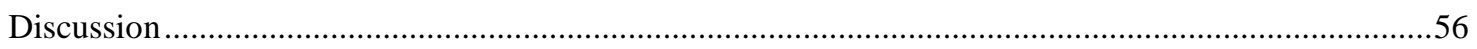

DETECTION OF PHOSPHATIDYLCHOLINE-COATED NANOPARTICLES IN ORTHOTOPIC

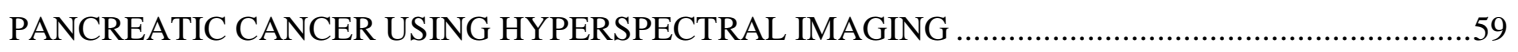

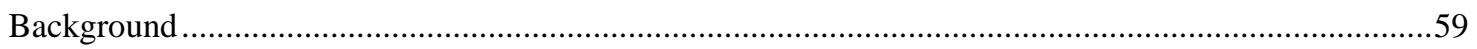

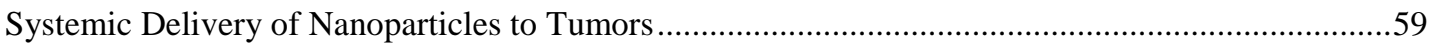

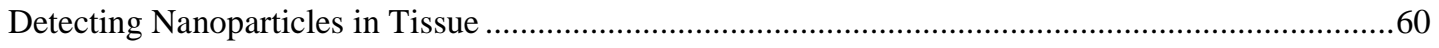

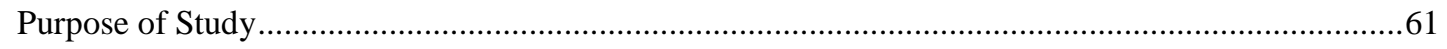

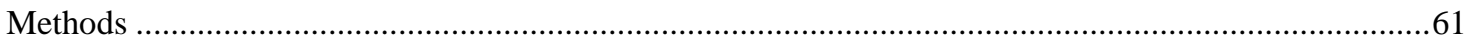

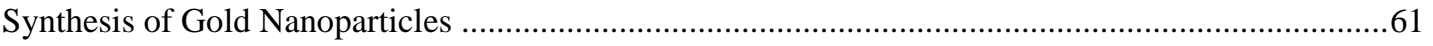

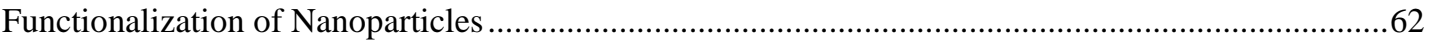

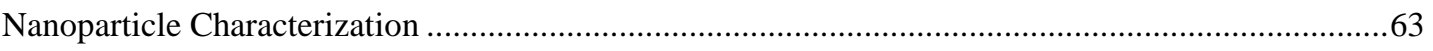

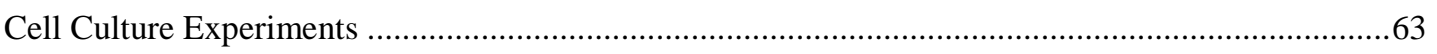

Human Pancreatic Cancer Orthotopic Xenograft Mouse Model ......................................................63

Mouse Euthanasia, Organ Resection, and Histological Processing ......................................................64

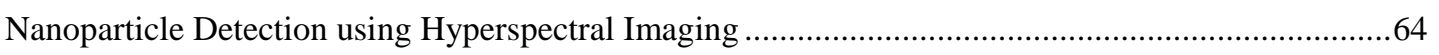

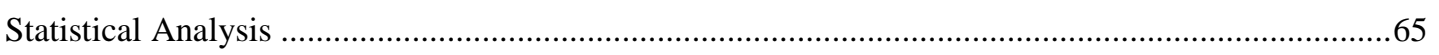

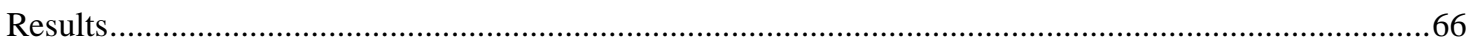

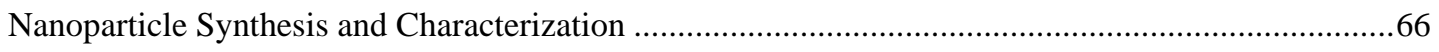

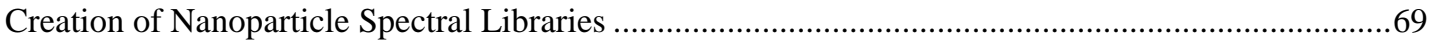

Evaluation of Nanoparticle Uptake and Distribution.....................................................................

Nanoparticle Accumulation in Liver, Spleen, and Pancreatic Tumor ................................................72

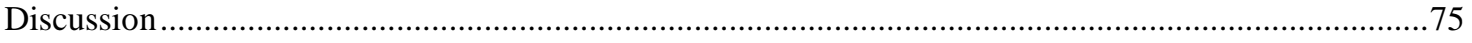

RELEASE KINETICS OF CISPLATIN AND PACLITAXEL FROM TWO AND THREE LAYER

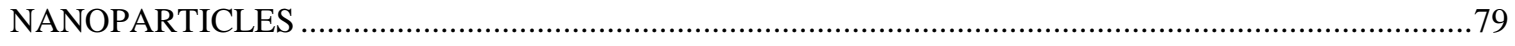

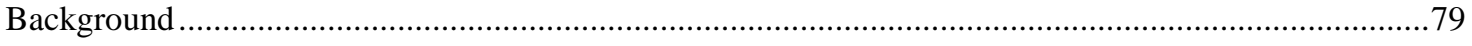

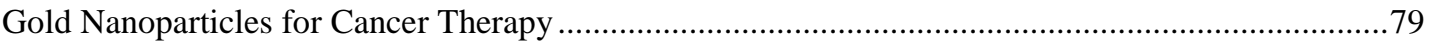

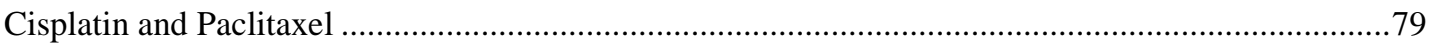

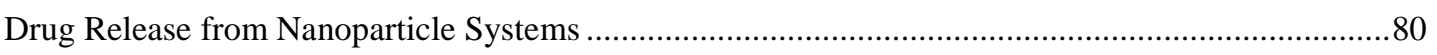

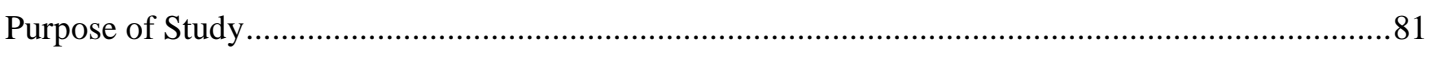

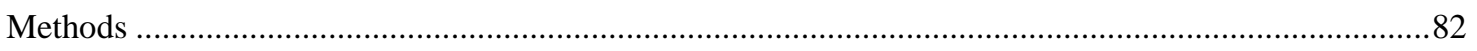

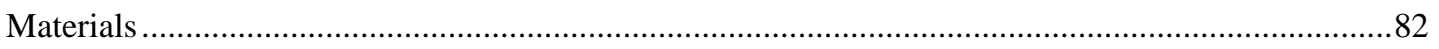

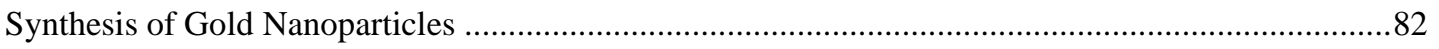




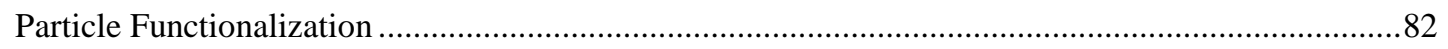

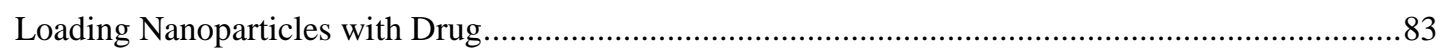

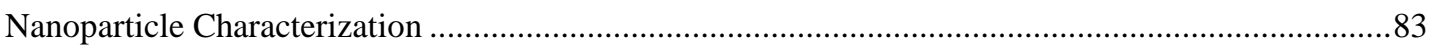

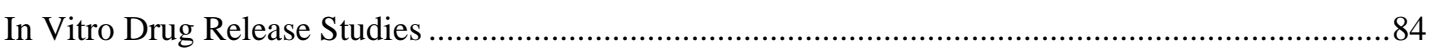

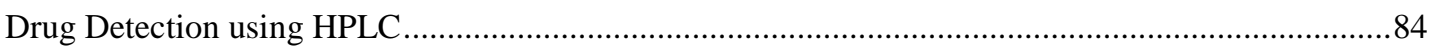

Determination of Drug Encapsulation Efficiency and Drug Loading Efficiency ................................. 85

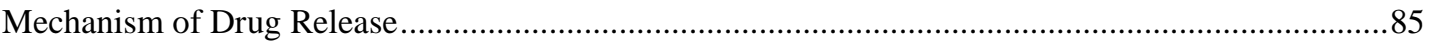

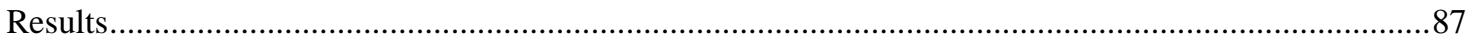

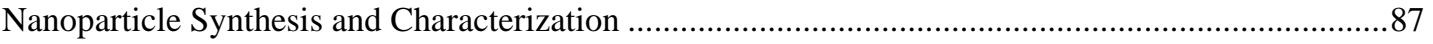

Drug Release from Two and Three Layered Gold Nanoparticles..................................................... 92

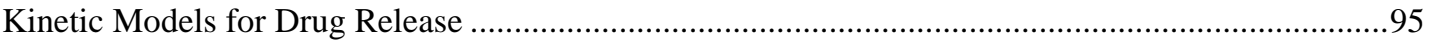

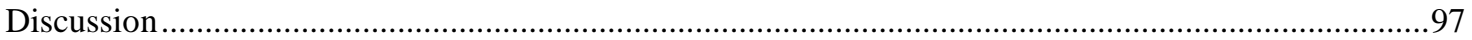

CYTOTOXICITY OF 2- AND 3- LAYER GOLD NANOPARTICLES IN 3D MODELS OF LUNG

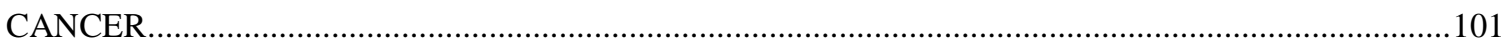

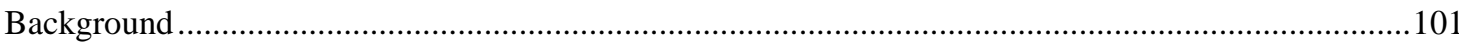

Chemotherapeutic Delivery to Solid Tumors …………..................................................................101

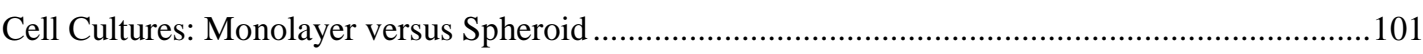

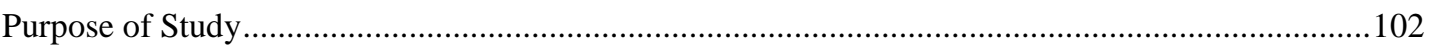

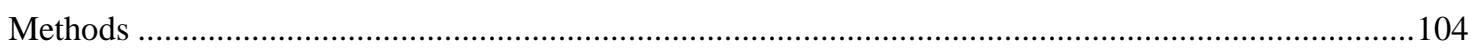

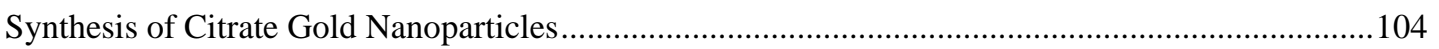

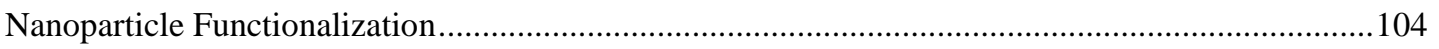

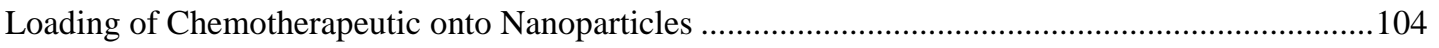

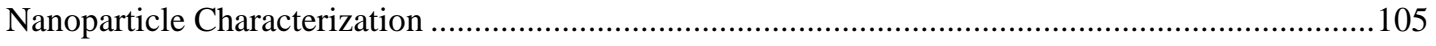

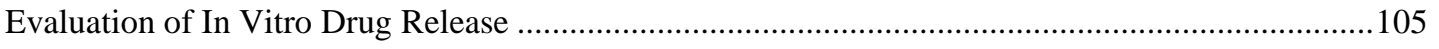

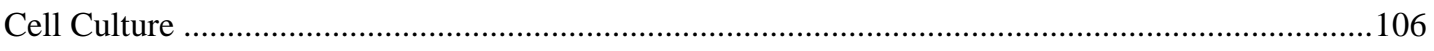

Evaluation of Free Drug Cytotoxicity in 2D Cell Cultures ...............................................................106

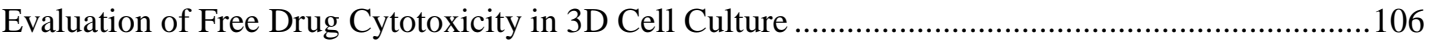

Evaluation of Drug Loaded Nanoparticles in 3D Cell Culture …....................................................... 107

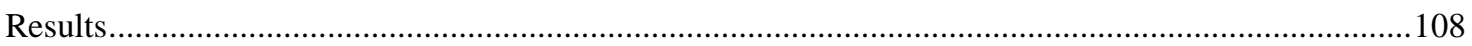

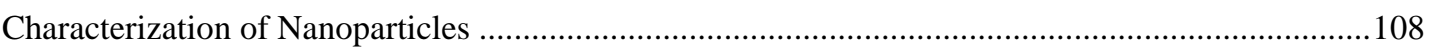

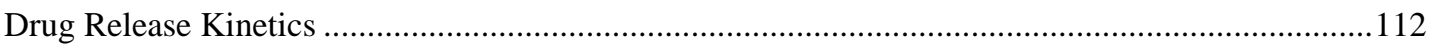

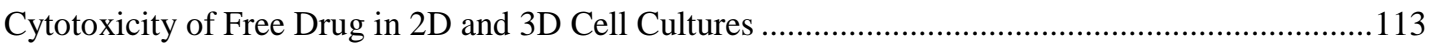

Cytotoxicity of Drug-Loaded Nanoparticles in 3D Cell Cultures ....................................................115

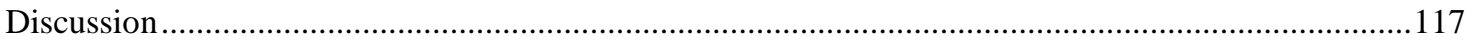

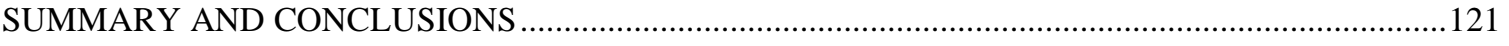

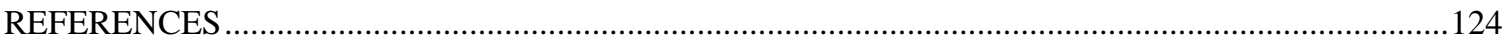




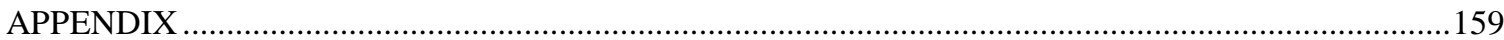

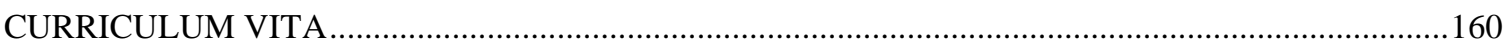




\section{LIST OF TABLES}

TABLE

PAGE

1. Determination of Nanoparticle Size and Wavelength.............................................. 45

2. Characterization Results of Two and Three Layer Gold Nanoparticles...............................91

3. Drug Incorporation Efficiency of Two and Three Layer Gold Nanoparticles...........................95

4. Rate Constants Obtained from Modeling Drug Release from Gold Nanoparticles.....................96

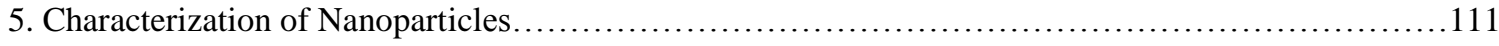

6. Cumulative Release of Drug at Various Time Intervals with Associated Loading Efficiency.............113 


\section{LIST OF FIGURES}

FIGURE

PAGE

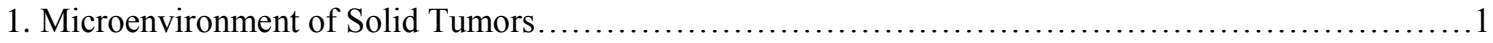

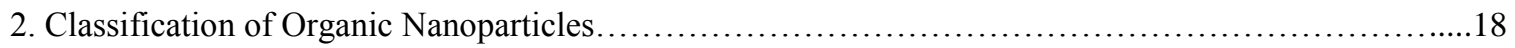

3. Classification of Inorganic Nanoparticles........................................................ 19

4. Illustration of Functionalized Gold Nanoshells.......................................................... 43

5. Characterization of Gold Nanoparticles by UV-Visible Spectrometry and Zeta Potential Analysis.......44

6. Determination of Surface Functionalizing using FTIR ......................................... 46

7. Scanning Electron Images of Functionalized Gold Nanoparticles.................................47

8. Nanoparticle Detection in 3D Cell Cultures through Silver Staining ..............................49

9. Accumulation of Gold Nanoparticles in 3D Cell Cultures by Silver Enhancement......................50

10. Detection of Nanoparticles in 3D Cell Cultures using Dark Field Microscopy......................52

11. Hyperspectral Imaging of Nanoparticles using CytoViva Imaging System........................52

12. Nanoparticle Distribution in 3D Cell Cultures Measured by Dark Field Microscopy....................54

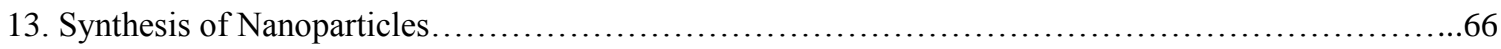

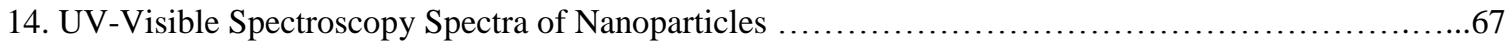

15. Hydrodynamic Radius of Nanoparticles Measured using DLS.....................................68

16. Surface Charge of Nanoparticles Measured using the Zetasizer......................................68

17. Hyperspectral Imaging of PC-coated Gold Nanoparticles........................................69

18. Nanoparticle Penetration in Liver, Spleen, and Pancreatic Tumor..................................71

19. Gold Nanoparticle Concentrations within Liver, Spleen, and Pancreatic Tumor.........................73

20. Nanoparticle Illustration of Synthesis and Functionalization...................................... 87

21. UV-Spectroscopy and Zeta Potential Analysis of Gold Nanoparticles.............................89

22. Hydrodynamic Size of Gold Nanoparticles in Solution........................................... 90

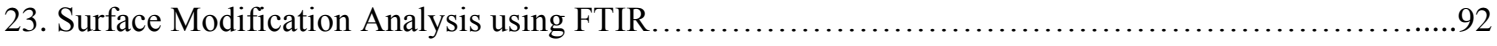


24. Drug Release from Functionalized Two and Three Layer Gold Nanoparticles......................93

25. First 24 Hours of Drug Release from Gold Nanoparticles.........................................94

26. Paclitaxel Release from Three Layer Gold Nanoparticles Fitted to Kinetic Models.................. 96

27. Synthesis of two- and three layered gold nanoparticles...........................................

28. Gold Nanoparticles Characterized using UV-Vis Spectroscopy...............................110

29. Scanning Electron Microscopy to Determine Size and Shape of Nanoparticles....................110

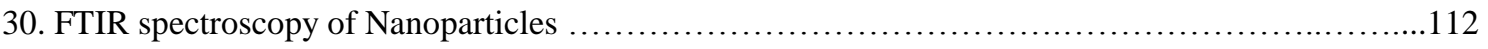

31. Cytotoxicity of Free Cisplatin and Paclitaxel in Three NSCLC Cell Lines..........................114

32. Cytotoxicity of Drug-Loaded Nanoparticles in Comparison to Free Drug in 3D Cell Cultures........116 


\section{CHAPTER I}

\section{INTRODUCTION TO NANOTHERAPEUTICS IN CANCER TREATMENT}

\section{Biological Barriers to Successful Treatment}

Several biological differences between the microenvironment found within solid tumors and that of normal tissue effectively limit the efficacy of chemotherapeutic drugs as well as nanotherapeutics. Noncancerous tissue contains organized vasculature providing oxygen, nutrients, and drug delivered systemically to all of the surrounding cells. Cancerous tissue presents multiple barriers to the successful delivery of oxygen, nutrients and therapeutics. These barriers are "hallmarks" of the microenvironment of solid tumors and include: (1) irregular and disorganized vasculature, (2) high interstitial fluid pressure (IFP), (3) low oxygen tension and hypoxia, (4) low extracellular pH, (5) and a dense extracellular matrix (ECM) (Figure 1) [1, 2].

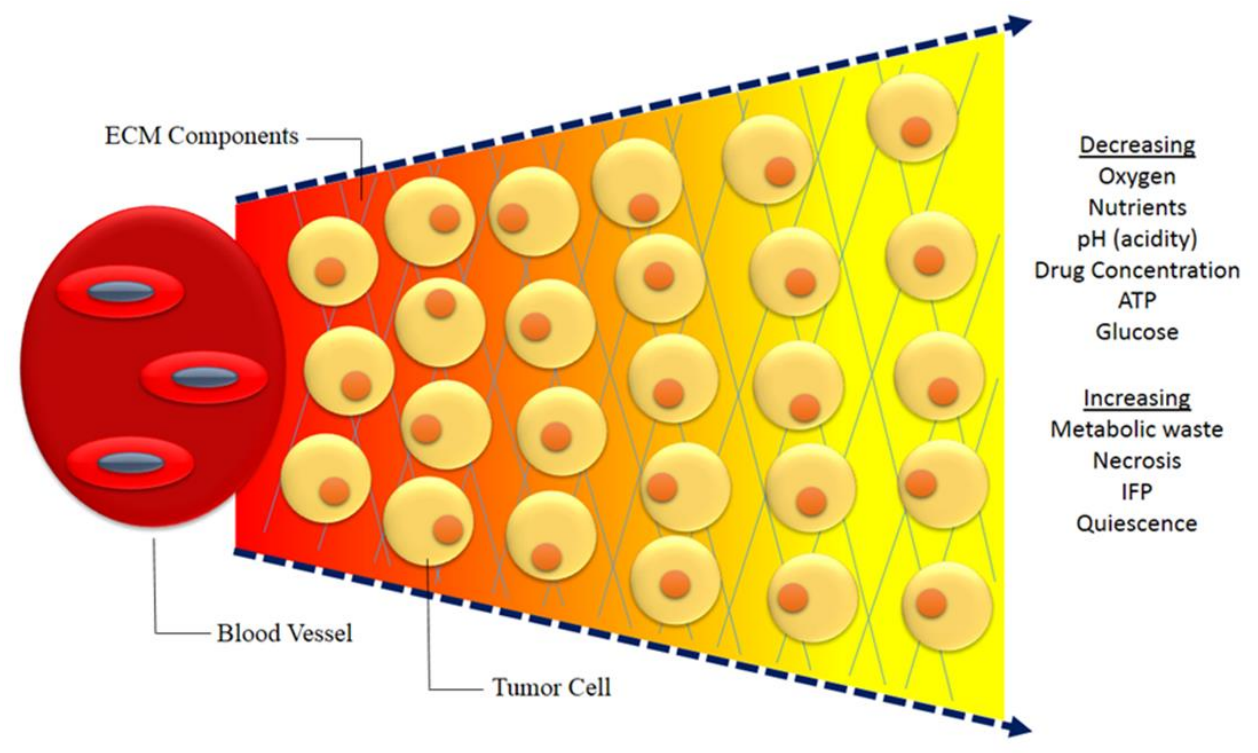


Figure 1. Microenvironment of Solid Tumors. Solid tumors elicit several characteristics different from healthy tissue primarily attributed to irregular vasculature. Cells distant from surrounding blood vessels will experience hypoxia as oxygen diffusion is limited, thus certain regions of tissue will have inadequate access to oxygen and other nutrients (e.g. glucose). Those cells distant from vasculature will also have impaired means of eliminating metabolic waste, resulting in an increased extracellular acidity. The cells distant from vasculature can become quiescent or necrotic due to the lack of nutrients. Also, an increase in IFP results from the irregular vasculature along with impaired lymphatic function. This increase can further decrease the diffusion of small molecules, effectively limiting the access of drugs and nanoparticles to the tumor.

The irregular and leaky vasculature in solid tumors arises from un-balanced regulation of proangiogenic and anti-angiogenic factors [3]. As a result, portions of tissue will become hyper-vascularized while other portions will become hypo-vascularized. Another leading contributor to the disorganized tumor vasculature are the disproportional mitotic rates between endothelial and tumor cells, as the latter proliferate much faster than endothelial cells making up the vasculature, thus leading to sporadic blood vessel formation [4]. As a consequence of irregular vasculature, malignant cells will become distant from surrounding blood vessels, possibly beyond the diffusion limit of oxygen $(100 \mu \mathrm{m})$. This results in the development of oxygen and nutrient gradients in which cells closer to vessels will receive most of the nutrients and proliferate, while cells distal to the vasculature will receive insufficient oxygen and nutrients, thus promoting cellular quiescence and even necrosis [5].

It has been well documented that solid tumors commonly possess elevated interstitial fluid pressure (IFP) in comparison to non-cancerous tissue, where the IFP is regulated to optimize fluid flow and nutrient transport from blood vessels to surrounding cells [2]. Whereas in normal tissue the IFP is slightly negative (-1 to $-3 \mathrm{~mm} \mathrm{Hg}$ ), in solid tumors it can range as high as 10 to $30 \mathrm{~mm} \mathrm{Hg}$ [6]. For example, the IFP of implanted A549 orthotopic solid tumors in mice were monitored in correlation with tumor size. As the tumor volume increased to $2000 \mathrm{~mm}^{3}$, IFP also increased from $2 \mathrm{~mm} \mathrm{Hg}$ to $4 \mathrm{~mm} \mathrm{Hg}$, with other cell lines exhibiting higher increases [7]. There are three factors that influence IFP in solid tumors, including: decreased function of blood vessels and lymphatics residing within the tumor microenvironment, osmotic forces that draw solute into the tissue, and contraction of the tumor stroma [1]. As the factors are 
synergistic, they can promote high solid tumor IFPs s [1]. Numerous studies have analyzed how increased IFP affects the therapeutic efficacy of drugs, with most studies showing a positive correlation between increased IFP and poor prognosis [8].

As the cells further away from vasculature become quiescent, a gradient of decreasing cell proliferation with increased distance from blood vessels will develop [9]. Quiescent cells have shown increased incidence of resistance to both chemotherapeutics and radiotherapy [10,11]. Reasons include:

(A) hypoxic cells are usually quiescent not allowing for cycle-dependent chemotherapeutics to be effective, (B) chemotherapeutics may be inactive in acidic or hypoxic microenvironments, and (C) cells may receive sub-optimal drug concentrations simply attributed to the distance from the nearest blood supply [4].

The acidic microenvironment of solid tumors can hinder the delivery of chemotherapeutics in multiple ways [12]. The low $\mathrm{pH}$ found in solid tumors is attributed to the build-up of metabolites such as lactic acid and carbonic acid in the extracellular space. As healthy non-cancerous cells excrete metabolites from the cellular cytoplasm to the interstitial space, surrounding vasculature and lymphatic ducts will eliminate the metabolites from the tissue. In cancerous tissue, insufficient vasculature and lymphatic processes hinder metabolite elimination, leading to their accumulation and resulting in acidic microenvironments. In accordance with the theory of ion trapping, drugs must be in an uncharged state in order to cross cellular membranes. Drugs such as doxorubicin are weak bases, which will become ionized in acidic environments, thus being unable to cross the cell membrane. As the amount of drug entering the cell decreases, the potential therapeutic action will also diminish [13]. Thus, the acidity of the microenvironment can significantly impact therapeutic outcomes.

Solid tumors can possess significantly more ECM proteins in comparison to non-cancerous tissue, resulting in decreased diffusivity of various nutrients and drugs. The ECM is the tissue outside of the cells that provides structural and biochemical support for the cellular structure. It is responsible for cell adhesion, cell-to-cell communication, and structural integrity with various proteins (e.g. proteoglycans, collagen, hyaluronic acid) and can have a large impact on drug delivery as the composition and quantity of ECM can drastically hinder the movement of both medium and large molecular weight proteins [4] due to size and charge constraints. For example, collagen possesses a slightly positive charge which may attract anionic 
nanoparticles or drugs, thus decreasing their delivery to malignant tumor cells residing further away from vasculature [14].

Besides limitations exhibited by the solid tumor, certain chemotherapeutic drugs have poor pharmacokinetic properties that can also limit their effectiveness. For example, certain hydrophobic drugs (e.g. paclitaxel) are difficult to use clinically due to deficient water solubility and high plasma protein binding [15]. Many compounds have been eliminated as potential drug candidates due to their unsuitable physiochemical properties. To overcome these issues, nanoparticles have been utilized as drug carrier molecules. Nanoparticles are candidates for the delivery of various compounds including chemotherapeutics, miRNAs, and imaging agents. For example, paclitaxel is currently available as a nanobased therapeutic albumin-bound drug (Abraxane) for the treatment of lung cancer [16]. 


\section{Delivering Nanoparticles to Solid Tumors}

Interest arose in the use of nanoparticles as potential drug carriers because they could be combined with previously developed biologically active compounds to improve drug delivery to tumors at increased concentrations in comparison to free drug. Properties of nanoparticles that make them candidates for therapeutic applications, include: (1) high tunability allowing for modification of properties such as shape, size, and surface charge, (2) ability to specifically target cancer cells, (3) ability to sporadically accumulate in tumor tissue via passive targeting, (4) ability to overcome physiochemical limitations of the drug itself, (5) controllable drug release profiles which can be modified for various cancers or treatment regimens, (6) ability to act as theranostic agents or the simultaneous delivery of diagnostic molecules and therapeutic agents for the concurrent imaging and treatment of disease, (7) ability to carry multiple drugs, and (8) ability to bypass intrinsic cellular resistance mechanisms for entry into cells, such as P-glycoprotein. Each of these properties will be discussed in more detail below.

(1) Nanoparticles are highly tunable, which makes them optimal for appropriate applications in medicine. Properties including conformational shape, maximum absorbance, surface charge, and hydrodynamic size can be chemically modified to enhance their efficacy. Conformational shape can have a crucial impact on cell uptake in vivo, as spherical nanoparticles have been shown to accumulate in tumor tissue better than rod-shaped nanoparticles. In 2013, Kolhar et al. showed that rod-shaped nanoparticles exhibited higher specific accumulation in lung endothelium in comparison to spherical-shaped particles [17]. The maximum absorbance is another modifiable property of nanoparticles, which can be chemically altered for purposes such as photoacoustic imaging or photothermal therapy. Investigators were able to monitor gold nanoparticles with an absorption peak around $780 \mathrm{~nm}$ as they circulated throughout systemic circulation using photoacoustic imaging [18]. Ren et al. was able to synthesize paclitaxel-loaded gold nanorods with a maximum absorbance near $780 \mathrm{~nm}$ and showed the feasibility of using these nanoparticles for both photothermal and chemotherapy efficacy in treating lung cancer [19].

Surface charge also has a large impact on the travel of nanoparticles within circulation and their diffusion through tissue. Cationic nanoparticles accumulate in tumor vasculature due to electrostatic interactions, but the cationic charge must be minimal for nanoparticles to extravasate from blood vessels $[20,21]$. It has been shown that neutral charged nanoparticles diffuse rapidly within tumor tissue in 
comparison to charged nanoparticles which may react with extracellular proteins such as hyaluronan or collagen $[14,22]$. The effect of nanoparticle size on tissue distribution has been extensively studied. Smaller nanoparticles (10-100 nm) exhibit enhanced diffusivity in tumor tissue as compared to larger nanoparticles, while larger nanoparticles $(100-400 \mathrm{~nm})$ are significantly sequestered due to the EPR effect. For example, liposomes ranging in size from 100-200 nm displayed enhanced tumor accumulation in vivo, as compared to smaller liposomes $(<100 \mathrm{~nm})$ and larger nanoparticles $(>200 \mathrm{~nm})$ [23]. Further studies have analyzed the diffusion of even smaller nanoparticles in tumor tissue, such as Huang et al. who examined the relationship of ultra-small gold nanoparticles and tumor accumulation using nanoparticles ranging in size from 2-15 nm. It was shown that 2 and $6 \mathrm{~nm}$ nanoparticles accumulate in tumor tissue after intravenous injection in higher concentrations than slightly larger nanoparticles $(15 \mathrm{~nm})$. They further analyzed the localization of these nanoparticles within individual cancer cells and the microenvironment of the tumor, illustrating that both 2 and $6 \mathrm{~nm}$ particles were distributed throughout the cytoplasm and nucleus of cancer cells, while larger nanoparticles were only found within the cytoplasm where they formed aggregates [24].

(2) The process of functionalizing nanoparticles refers to the chemical addition of various surface modifications, such as proteins, peptides, antibodies, or other compounds that can be used to enhance the delivery of nanoparticles to the tissue of interest. By specifically targeting cancer cells, systemic off target toxicity that is commonly associated with current chemotherapeutics can be minimized. This process has been termed "active targeting." Previous studies have targeted nanoparticles to cell surface receptors commonly up-regulated in cancer cells, such as EGFR [25-28]. Kao et al. recently demonstrated that gold nanoparticles targeted to EGFR in A549 cells exhibited higher radioactivity retention in the tumor after intravenous injection, as compared to non-targeted nanoparticles [25]. Additional groups such as Peng et al. developed EGFR-targeted heparin nanoparticles loaded with cisplatin, which were shown to significantly increase the intracellular concentration of drug in H292 NSCLC cells as compared to cisplatin alone. These nanoparticles showed enhanced pharmacokinetics and bio-distribution through systemic circulation improving antitumor activity without weight loss or associated nephrotoxicity [28]. As nanoparticles possess high surface area to volume ratio due to their small size and shape, studies have shown the feasibility of attaching multiple targeting ligands onto a single nanoparticle, thus increasing the odds of cellular interactions with the targeted tissue. For example, Rangger et al. developed liposomes targeted 
with two peptides: an arginine-glycine-aspartic acid peptide and a neuropeptide. While the additional surface modification was predicted to increase tumor accumulation of the liposomes, they found no additional tumor uptake with the dual-targeted liposomes compared to single targeted liposomes [29]. This study demonstrated an important concept; while adding multiple targeting ligands seems very useful, the activity of the targeting ligands may not provide a synergistic effect on targeting capabilities and tumor accumulation.

(3) Irregular vasculature in tumor tissue hinders the delivery of nutrients to some of the cells residing in the tumor. The same holds true for freely circulating drugs, in which cells distant from vasculature will receive minimal amounts of chemotherapeutics leading to suboptimal drug concentrations. Nanoparticle accumulation in tumor tissue is only a small fraction of the loading dose. Previous studies have shown that most injected nanoparticles will accumulate in the liver (40-50\%), spleen (24-40\%), lungs, kidneys, and heart. Only 1-5\% of injected nanoparticles will accumulate in the tumor tissue, which can ultimately lead to therapeutic failure [30]. For this reason, coating nanoparticles with polymers such as poly(ethylene) glycol (PEG) has been used to enhance the bioavailability. It was shown that nanoparticles coated with PEG exhibit an increase circulation time in vivo, over $2 \mathrm{X}$ that of non-coated nanoparticles [31, 32]. Passive targeting of nanoparticles is based upon the observation that nanoparticles will accumulate in tissue surrounding the leaky vasculature as a result of wide fenestrations of the endothelial layer and reduced lymphatic draining $[2,33]$. This phenomenon has been termed the enhanced permeability and retention effect (EPR), which was first described by Hiroshi Maeda in 1985 [34, 35]. Scientists discovered that increasing the circulation time of nanoparticles in vivo led to higher accumulation in tumor tissue, as the EPR effect was time-dependent.

(4) The biological effect of a drug is significantly dependent upon its pharmacokinetic properties. Many chemotherapeutics have substandard pharmacokinetic profiles which limit their travel throughout systemic circulation, due to factors such as rapid metabolism or elimination. Major issues associated with poorly water-soluble compounds include: poor bioavailability, lack of dose-response proportionality, suboptimal dosing, use of harsh excipients, use of basic or acid conditions to enhance solubility, uncontrollable precipitation after dosing, and noncompliance by the patient due to dosing strategies [36]. The limited pharmacokinetic properties can be overcome by using nanovectors as a delivery mechanism. 
As nanoparticles are synthesized with tunable pharmacokinetic properties, drugs will be delivered to the tumor tissue based upon the pharmacokinetic properties of the nanoparticle. For example, paclitaxel is one of the most effective FDA-approved chemotherapeutic drugs due to its anti-cancer activity and relatively low $\mathrm{EC}_{50}$, yet minimal water solubility and harsh solvents can hinder efficient delivery and increase the risk of severe toxicity. The nano-based formulation of albumin-bound paclitaxel has shown enhanced effectiveness, increased survival rates, increased time to disease progress, and decreased systemic toxicity [37].

(5) Once drugs are loaded onto nanoparticles, their release profiles can be modified for the treatment of specific diseases. Drug release from nanoparticles is dependent upon several factors including $\mathrm{pH}$ of the surrounding microenvironment, interactions between the drug and nanoparticle (e.g. covalent linkage or non-covalent linkage), and the physiochemical properties of the nanoparticles. For example, Yin et al. synthesized curcumin-loaded nanoparticles with three different amphilic methoxy PEG (mPEG)polycaprolactone (PCL) block copolymers varying in chain length. They showed that mPEG10k-PCL30k had the highest loading efficiency and most sustained drug release profile of the three polymers. Results from this study illustrated that various alterations in polymer size could have substantial effects on drug release kinetics, so constructing nanoparticles specifically for certain release profiles is feasible and worthwhile [38]. Through surface modifications, rapid and slow drug release patterns can be achieved. The most common method for analyzing drug release kinetics from nanoparticles is by dialysis, along with analysis by UV-spectroscopy or high performance liquid chromatography (HPLC) [39]. Li et al. loaded doxorubicin in anionic methoxy PEG-b-poly(L-glutamic acid) nanoscale complexes that exhibited $\mathrm{pH}$ responsive drug release and significantly prolonged blood circulation time in comparison to drug alone in nude mice with A549 NSCLC xenografts [40]. By prolonging circulation time of the nanoparticle, there were more opportunities for the nanoparticle to localize in the tumor tissue as it continuously re-circulated throughout the body while avoiding the healthy tissue.

(6) While loading drugs onto nanoparticles can provide enhanced therapeutic responses, this concept was extended to loading imaging agents along with drugs. The development of such particles was a direct result of scientists wishing to visualize the effects of anticancer treatments. New imaging methodologies along with novel theranostic nanoparticles made this process possible. For example, 
photoacoustic imaging with multispectral optoacoustic tomography (MSOT) has enabled monitoring the systemic circulation of gold nanoparticles in real-time [41-43]. Magnetic resonance imaging (MRI) has been most extensively utilized with theranostic nanoparticles. Patel et al. recently developed theranostic nanoparticles containing multiple modifications including: PEG-coating to increase systemic circulation, a CREKA peptide for active targeting to lung cancer, and a cytotoxic compound known as c-substituted diindolymethanes (CIM-C-pPhC6H5) for therapeutic activity. These newly developed nanoparticles exhibited a 40-fold increased transport in tumor vasculature compared to control nanoparticles [44].

(7) Due to the high surface area to volume ratio, multiple targeting ligands and therapeutic agents can be loaded onto a single nanoparticle. This is advantageous since chemotherapeutics regimens commonly consists of two or more drugs in combination. Aratula et al. developed mesoporous silica nanoparticles with two anti-cancer drugs (cisplatin and doxorubicin) and two siRNA molecules targeted to MRP1 and BCL2 mRNA to decrease cellular resistance. Using the luteinizing hormone releasing peptide (LHRH) as an active targeting ligand, they were able to enhance tumor accumulation, as the siRNA suppressed cellular resistance to the anticancer drugs, and the anti-cancer agents exhibited enhanced therapeutic effect for the treatment of NSCLC in vivo [45]. Another group, Liu et al., encapsulated both doxorubicin with paclitaxel and doxorubicin with rapamycin into magnetic mesoporous silica nanoparticle to demonstrate the feasibility of loading both hydrophobic and hydrophilic drugs onto the same nanoparticle. The nanoparticles showed high internalization and enhanced efficacy in A549 NSCLC cells [46]. By loading multiple drugs onto single nanoparticles, patient compliance could be improved as the number of necessary drug doses could be decreased.

(8) Drug resistance is a critical hindrance to successful chemotherapeutic treatment. Multidrug resistance (MDR) has been the most extensively studied mechanism in which cancer cells become immune to multiple chemotherapeutics [47]. There are both cellular and physiological factors that can alter drug resistance. Cellular responses involved in drug resistance include altered molecular targets, increased drug metabolism, genetic defects, reduced apoptosis, and up-regulation of efflux pumps [48]. Physiological mechanisms of drug resistance include cell-cell interactions, increased IFP, acidic microenvironment, hypoxic or necrotic regions in tissue, and irregular tumor vasculature [48]. Efflux pumps such as Pglycoprotein are commonly associated with increased drug resistance to multiple classes of 
chemotherapeutics including doxorubicin, vinca alkaloids, and taxols $[49,50]$. Using nanoparticles, some mechanisms of drug resistance can be overcome. For example, Cuvier et al. showed that both sensitive and resistant cells would uptake the same amount of doxorubicin if delivered via nanoparticles [51]. Other groups have looked to target intracellular apoptotic mechanisms, such as Bcl-2, which is a protein that inhibits downstream apoptotic pathways and is up-regulated in most solid tumors. To overcome this drug resistance mechanism, Bcl-2 siRNAs has been encapsulated to decrease drug resistance in vivo [52-54]. For example, Saad et al. developed liposomes containing two different siRNAs, Bcl2-siRNA and MRP1siRNA, and doxorubicin for use in MDR lung cancer cells. Results showed effective co-delivery of doxorubicin and siRNAs with up to $95 \%$ cell-death induction, with high suppression of cellular resistance [55]. 


\section{Lung Cancer Facts and Statistics}

While other cancer cell lines are utilized in this study, lung cancer is the primary focus. Lung cancer remains a lethal ailment in the United States in spite of recent treatment advances. Treatment options are disease-dependent, and can include surgical intervention, radiation therapy, chemotherapeutics, and select targeted therapies. While these modalities have been shown to improve life expectancy and enhance quality of life, late onset of clinical symptoms frequently yields diagnoses resulting in incurable advanced disease. Patients with inoperable disease depend entirely on currently available chemotherapeutics, yet rapid resistance, miniscule therapeutic windows, excessive off-target toxicity, and substandard pharmacokinetic properties make many of these drugs therapeutically suboptimal. Even for patients diagnosed at early stages, undergoing chemotherapy and radiation after surgical removal of the primary tumor commonly causes severe adverse effects related to off-target drug toxicity.

A remarkable shift occurred in the late part of the 20th century as incidences of lung cancer grew exponentially, eventually surpassing the incidence rates of all other forms of cancer. Along with increased prevalence, associated deaths transcend all other cancers, rendering lung cancer as the leading cause of cancer-related death in both men and women in the United States $[16,56]$. While incidence and mortality rates have declined for men since the 1980s, women have only recently begun to see a decline is death rates. In 2013, estimates show that lung cancer will account for approximately $14 \%$ of newly diagnosed cancers (228,190 cases) and nearly $27 \%$ of cancer-related deaths (159,480 deaths) [56]. Compared to other forms of cancer, lung cancer accounts for twice the deaths associated with breast cancer in women, and three times that of prostate cancer in men [57]. Overall, recent statistics show that men have a 1 in 13 chance of developing lung cancer throughout their lifetime, while women have slightly better odds of 1 in 16 [56]; these statistics include both smokers and non-smokers. When considering the population of nonsmokers, male smokers are approximately 23 times more likely and female smokers are 13 times more likely to get lung cancer in their lifetime [56].

Despite major advances in combatting this disease, overall 5-year survival rates for all types of lung cancer remain at a dismal 16\% [56]. While 5-year survival rates for localized disease is nearly $52 \%$, virtually $70 \%$ of patients present with regional or distantly advanced disease, which have 5 -year survival rates of $14 \%$ and $1 \%$, respectively $[16,56]$. For such patients, current treatment options provide a median 
survival of 10-12 months, indicating that early detection is critical for enhancing survival [16]. The majority of cancer cases manifest in individuals $>59$ years of age, as the probability of developing lung cancer increases proportionately with age. For example, the overall probability of developing lung cancer while 40-59 years of age is 1 in $120(0.9 \%), 60-69$ years of age is 1 in $51(2.1 \%)$, and > 70 years of age embodies the highest odds at 1 in $17.5(5.7 \%)$ [58]. As with many other cancers, racial disparity is evinced as African Americans experience higher incidence and mortality rates from lung cancer; however, recent data suggest that this trend will diminish within the next $40-50$ years as smoking cessation offerings have increased in African American communities [59]. These statistics highlight the essential need for novel treatment options to enhance quality of life and increase survival.

Lung cancer is considered the most preventable form of cancer, as positive correlations between cigarette smoking and increased incidence rates have been studied extensively since the 1950s. The first acknowledgement that lung cancer could be associated with cigarette smoking came from Sir Richard Doll and Austin Hill in 1950 [60]. It was not until 1964 that the U.S. Surgeon General declared cigarette smoking as the causative agent for most incidences of lung cancer, while also declaring that increased risk of lung cancer was proportional to quantity and duration of exposure [61]. It is estimated that approximately 80-90\% of lung cancer deaths in the U.S. can be directly associated with tobacco use, while other cases have been attributed to environmental factors (e.g. asbestos) [62,63]. Studies have shown that smoking duration has an essential role in estimating potential risk for developing lung cancer, as the risk diminishes each year the person remains smoke-free. For example, a 65 year old male who quits smoking at 50-54 years old will decrease his relative risk for developing lung cancer by approximately 70\% [64]. Overall, it is estimated that tobacco users who continuously smoke throughout their lifetime have 20-fold greater odds of developing lung cancer compared to individuals who successfully abstain from smoking for at least one year [64].

While exposure to tobacco smoke accounts for a large portion of lung cancer incidences, there are several other risk factors for lung cancer such as passive smoking (second-hand smoke or environmental exposure), asbestos exposure, contact with certain metals (e.g. chromium and arsenic), and radiation [56]. Previous studies showed significant synergistic interactions among risk factors for lung cancer. For example, smokers who are exposed to asbestos or radiation have much higher odds of developing lung 
cancer than smokers not exposed to asbestos or radiation as the two risk factors are synergistic [65]. Current estimates suggest that $\sim 10 \%$ of lung cancer cases can be linked to occupational exposure [66], making it the most common workplace-associated cancer [67]. Other risk factors such as contact with certain metals and radiation contribute a small number of incidences each year $(<1 \%)$ [68]. It is important to remember that only a small portion of lung cancer cases can be associated with individuals not exposed to tobacco or other carcinogens.

There are four major types of lung cancer based upon tissue morphology, including adenocarcinoma, squamous cell carcinoma, large cell carcinoma, and small cell carcinoma. The first three types have similar prognosis and treatment options, thus encompassing the non-small cell lung cancer (NSCLC) category. Small cell carcinoma is the only form of lung cancer categorized as small cell lung cancer (SCLC). NSCLC accounts for approximately $84 \%$ of yearly diagnosed cases, while SCLC accounts for the remainder (15\%) [56]. NSCLC is known to have a much slower growth rate and doubling time (i.e. the time it takes a tumor to double in size) (184 days) as compared to SCLC (86.3 days), [69]. Adenocarcinoma is the most predominant form of lung cancer accounting for nearly $50 \%$ of diagnosed cases, and is known for rapid metastases to other portions of the lung, liver, bone, adrenal glands, kidneys, and CNS [70]. Adenocarcinomas possess vast amounts of heterogeneous tissue [71]. Currently, an increased trend in adenocarcinoma has been seen in women and non-smokers, yet it remains most predominant in males $<50$ years old and women of all ages $[61,72]$.

The second most common form of NSCLC is squamous cell carcinoma, which accounts for $30 \%$ of cases diagnosed cases each year. Squamous cell carcinomas tend to involve the central airways and are known for metastasizing late in the disease process, thus having better prognoses in comparison to adenocarcinomas $[73,74]$. Lastly, nearly $10 \%$ of diagnosed NSCLC cases are classified as large cell carcinoma and are undifferentiated tumors with histological features atypical of other NSCLC types [73]. Small cell carcinomas account for $\sim 15 \%$ of all incidences of lung cancer in the United States. This type is very aggressive, often metastasizing before diagnosis (60-70\% of cases) and leading to scarce treatment options and dismal survival rates [75]. Treatment for patients with limited stage SCLC who undergo radiation and chemotherapy have a $20-25 \%$ cure rate, yet extensive stage SCLC is incurable [76]. The 
overall 5-year survival rate is much lower for SCLC compared to NSCLC, at $6 \%$ and $18 \%$, respectively [56].

Early detection is critical for successful treatment, yet only 15\% of cases are localized at diagnosis with the other $85 \%$ of diagnosed cases being regional or metastatic [77]. Overall, lung cancer is not easy to diagnose early due to the late onset of associated symptoms, along with other factors including concurrent respiratory problems that may mask cancer-related symptoms. The most common symptoms associated with lung cancer include cough, dyspnea, chest pain, sputum production, and hemoptysis [73]. It is not uncommon for physicians to overlook these symptoms in patients with histories of tobacco use, as these symptoms are often associated with on-going chronic pulmonary and cardiovascular disease. Advanced disease can cause other non-pulmonary symptoms such as neurological deficits, liver dysfunction, or bone pain, and these symptoms may be difficult to diagnosis early [73].

Common methods for detecting lung cancer include chest x-rays, computed tomography (CT) scan, and positron emission tomography (PET). Historically, studies have shown that chest radiography and sputum cytology do not reduce lung cancer mortality in comparison to the usual care provided by primary care physicians [78]. While integrated CT-PET has shown promise in comparison to either CT or PET alone in staging NSCLC $[79,80]$, there has been renewed interest in CT screening for at-risk patients with a $20 \%$ survival improvement over chest $\mathrm{x}$-rays [81]. If needed, physicians may request further tests such as magnetic resonance imaging (MRI), bronchoscopy, or needle biopsy [82]. Upon surgical resection, pathological examination of tumor biopsy is performed to confirm diagnosis and determine the stage of the disease.

Staging of NSCLC is performed using the TNM staging classification from the World Health Organization. The classification scheme is based upon the primary tumor size and extent $(\mathrm{T})$, regional lymph node involvement $(\mathrm{N})$, and presence or absence of distant metastases $(\mathrm{M})$ [83, 84]. Like most other cancers, there is a simpler four stage grouping system which states that stage I refers to tumors confined to the lung without lymphatic spread, stage II refers to large tumors with ipsilateral peribronchial or hilar lymph node involvement, stage III refers to the inclusion of other lymph node and regional involvement, and stage IV refers to tumors with distant metastases [85].Staging of SCLC is performed using a two-stage method by the Veterans Administration Lung Cancer Study Group, known simply as limited and extensive. 
In this grouping system, limited stage is defined as being confined to the primary tumor or surrounding lymph nodes, while extensive stage is not.

Currently available treatment options include surgical intervention, radiation therapy, chemotherapy, and select targeted therapies. Surgical resection of the tumor remains the most effective treatment, yet is not feasible in many cases. In these circumstances, chemotherapy, radiotherapy, and targeted therapies have been shown to increase survival rates and improve quality of life. While chemotherapeutics can be life-saving, their toxicity can evoke adverse effects such as arthralgia, pyrexia, rigors, hematuria, convulsions with the platinum-containing chemotherapeutic cisplatin, changes in color vision, and unusual lethargy [86]. While adverse effects caused by these chemotherapeutics can be debilitating, they remain in clinical use due to a lack of better alternatives.

If left untreated, most patients with NSCLC will die within one year of diagnosis. The first line treatment for stage I and II NSCLC is surgery, either lobectomy or pneumonectomy, depending upon stage and lymph node involvement. For those individuals who undergo surgery, $60-70 \%$ of patients with stage 1 and $40-50 \%$ of patients with stage II NSCLC will survive for at least 5 years after surgery with no sign of recurrence [87]. Adjuvant therapy using chemotherapeutics or radiotherapy in early stage resectable NSCLC has been debated, yet several large studies have shown benefits in survival. For example, the International Adjuvant Lung Cancer Trial (IALT) showed a 17\% improvement in disease-free survival with a $4 \%$ increase in overall survival for patients receiving platinum-based adjuvant therapy in comparison to patients receiving no adjuvant therapy [88].

NSCLC patients who present with advanced; non-resectable disease at the time of diagnosis depends on chemotherapy as the first line of treatment. Chemotherapy can normally provide an extra 2-4 months of life expectancy with $10 \%$ of patients surviving 12 months [87, 89]. First line chemotherapy treatment for NSCLC includes a doublet combination of a platinum-containing compound (cisplatin or carboplatin) with an additional non-platinum containing compound, such as gemcitabine, paclitaxel, docetaxel, or vinorelbine [90]. First line treatment is normally administered for 4-6 cycles in patients benefiting from the therapy, after which second line treatment will be administered. Second line chemotherapy for NSCLC includes docetaxel, pemetrexed, and erlotinib, yet there is no consensus regarding the optimal second-line treatment [91]. Agents such as erlotinib and gefitinib depended upon the 
over-expression of epidermal growth factor receptor (EGFR) by lung cancer cells, by acting as tyrosine kinase inhibitors. Both of these drugs have shown antitumor activity for patients with advanced recurrent NSCLC, along with low toxicity and enhanced quality of life $[92,93]$.

SCLC is more responsive to chemotherapy and radiotherapy in comparison to other forms of lung cancer [76]. While early stage NSCLC patients will undergo surgery for tumor resection, SCLC tumors are rarely removed due to aggressive growth rates [94]. Combination therapy using radiotherapy with chemotherapy has increased median survival rates for both limited and extensive SCLC by 14-20 months and 9-11 months, respectively in comparison to chemotherapy alone [94]. Since SCLC tumors are normally very radiosensitive, radiotherapy is used in combination with chemotherapeutics as first line treatment. As with NSCLC, doublet administration of chemotherapeutics is superior to single drug administration. For SCLC, there are fewer chemotherapeutics available; first line agents include cisplatin, carboplatin, etoposide, irinotecan, and topotecan. The most common combinations are cisplatin with etoposide, carboplatin with etoposide, cisplatin with irinotecan, and carboplatin with irinotecan [94]. Second line treatment of SCLC includes the agents ifosfamide, paclitaxel, docetaxel, and gemcitabine, with studies showing that patients who relapse after first line treatment have a mean survival of 4-5 months [94]. 


\section{Nanoparticles for Lung Cancer}

The term "nanomedicine" refers to the use of nanoparticles, typically $10-1000 \mathrm{~nm}$ in size, for the treatment of various diseases including lung cancer. Nanotherapeutics can enhance the delivery of vital chemotherapeutics and other agents to solid tumors by passively and actively targeting tumor tissue compared to freely circulating drug, thus increasing the drug concentration within cancerous tissue. Once in the target vasculature, nanoparticles accumulate in the tumor tissue due to a phenomenon known as EPR effect. In particular, in the treatment of lung cancer, nanoparticle delivery has been extensively studied using two routes of administration, inhalation and intravenous. Inhalation minimizes potential adverse effects as the particles are delivered mainly to the lung tissue.

The field of nanotherapeutics has grown exponentially since the mid 1900's. While much progress has been made, the use of nanoparticles in vivo still invokes unanswered concerns about potential toxicity and therapeutic efficacy. Although nanoparticles may be candidates for improved cancer therapy, more definitive studies regarding effective dose requirements and potential toxicity concerns are required. The numerous types of nanoparticles that have been synthesized can ultimately be divided into two categories, organic and inorganic nanoparticles.

Organic nanoparticles are those containing carbon residues such as carbon nanotubes, quantum dots, dendrimers, liposomes, and polymeric nanoparticles, as shown in Figure 2. Inorganic nanoparticles are particles containing no carbon residues and include magnetic nanoparticles, metallic nanoparticles, nanoshells, and ceramic nanoparticles as seen in Figure 3. Each type of nanoparticle will be discussed in detail illustrating their applications within the past five years in the diagnosis and treatment of lung cancer. 


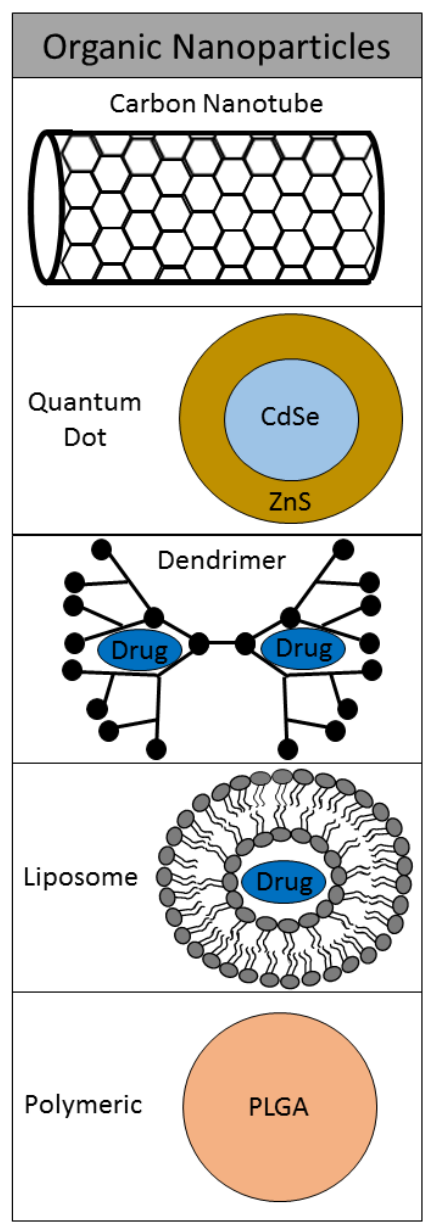

Figure 2. Classification of Organic Nanoparticles. Organic nanoparticles contain carbon residues within their structure and can be divided into five categories: carbon nanotubes, quantum dots, dendrimers, liposomes, and polymeric nanoparticles. Carbon nanotubes contain numerous 6-carbon rings that align next to each other and fold over to make a tube structure, capable of traversing cells without disturbing cell membranes. Quantum dots are small nanoparticles with cadmium cores and metallic shells shown to be effective imaging agents as they are brighter and more stable than most organic dyes. Dendrimers are branched macromolecules that can be precisely controlled during synthesis and capable of carrying large drug loads. Liposomes are closed spherical phospholipid bilayers that are non-toxic and biodegradable in vivo. Polymeric nanoparticles are colloidal particles that are modified with biodegradable polymer matrices to enhance circulation time and overall drug delivery. 


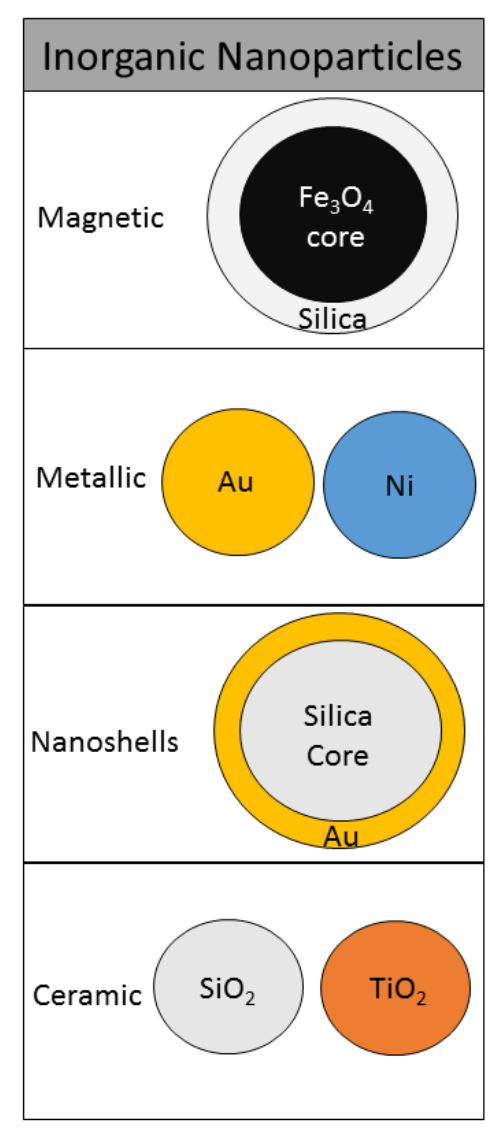

Figure 3. Classification of Inorganic Nanoparticles. Inorganic nanoparticles do not contain any carbon residues within their structure. Inorganic nanoparticles can be divided into four groups including magnetic nanoparticles, metallic nanoparticles, nanoshells, and ceramic nanoparticles. Magnetic nanoparticles have an inner core, most commonly of iron oxide, with an outer of shell of silica or other ceramic molecules. These nanoparticles can potentially be used for magnetic hyperthermia, a method of destroying tumor cells using heat. Metallic nanoparticles are composed of a solid metal such as gold or silver. Gold nanoparticles have been used extensively in research and can be functionalized with various targeting ligands or loaded with drugs for therapeutic action. Nanoshells consist of two layers similar to magnetic nanoparticles. For silica-gold nanoshells, there is outer layer of gold that surrounds a hollow silica sphere. Nanoshells are highly tunable as the thickness of the layer of gold surrounding the silica is modified. Ceramic nanoparticles are made from biocompatible materials such as silica or titania and exhibit enhanced biological stability and water solubility. 


\section{Organic Nanoparticles for Lung Cancer}

Carbon nanotubes (CNTs) have been studied in several biological applications including targeted delivery of vaccines, genes, siRNA's, and chemotherapeutics to tumors. Research has shown that CNTs transverse cell membranes without perturbing them, thus CNTs have the ability to delivery drugs into cells [95-97]. CNTs are produced by rolling up one or multiple layers of graphite sheets, which align as cylindrical tubes having diameters within the nanometer range [98]. They are noted for possessing strong material strength, along with high electrical and thermal conductivity due to their small size and mass [99]. The large surface area to volume ratio that is exposed to the outside environment makes them optimal for biological modification allowing for tumor-specific targeting, drug loading, and fluorescent tracking [100]. While the use of CNTs in lung cancer is feasible, many groups have shown that long-term exposure of CNTs can cause severe toxicity. For example, it was shown that CNTs can induce malignant transformation, tumorigenesis, and mitotic disruption of lung cells [101, 102]. Similarly, a study by Lohcharoenkal et al. showed a positive correlation between CNT exposure and mesothelioma. This group was further able to assess the mechanism of toxicity, which was linked to matrix metalloproteinase-2 (MMP-2), an enzyme involved in the breakdown of ECM [103].

Applications for CNTs in treating lung cancer have been explored in the last five years with positive results. Das et al. modified CNTs with the Technitium-99m radionucletoide, an Alexa-fluor fluorochrome, folic acid as a targeting molecule, and methotrexate as an active anticancer agent. This study showed a 19-fold tumor specific accumulation increase of the multi-functionalized CNTs in comparison to drug alone, proving that multi-functional nanoparticles could display better benefits in comparison to previously designed nanoparticles [104]. To further explore the use of multiple surface modifications, Datir et al. functionalized CNTs with hyaluronic acid and the anticancer agent doxorubicin, along with the same radionucleotide and fluorochrome as Das et al. for use in the A549 cell line. This study showed an increase in efficacy by 3.2-fold in comparison to drug alone with $\mathrm{pH}$-specific doxorubicin release [105]. The potential of CNTs acting as co-therapeutic agents for various chemotherapeutics was also demonstrated by Arya et al. through the utilization of graphene oxide CNTs for potentiating the efficacy of paclitaxel [106]. Minati et al. developed multi-functional carbon nanotube/gold hybrids that encapsulated doxorubicin for the treatment of A549 lung cancer cells. Results from this study showed that nanoparticles exhibited a large 
absorption band in the NIR-region making it an ideal imaging agent, along with enhanced cellular uptake of doxorubicin [107]. Further studies have analyzed the use and possible toxicity associated with carbon nanotubes in lung cancer including [108-114].

Quantum Dots (QD's) have semiconductor properties, also known as quantum-confinement, providing capability to emit fluorescence from the visible to near infrared wavelength [115]. Along with fluorescence properties, their small size makes them viable candidates for diagnostic purposes as they can remain in circulation for extended periods of time. QD's are synthesized from two atoms from the II/VI or III/V group of elements in the periodic table [116-119]. Some of the most widely employed quantum dots include $\mathrm{Cd} / \mathrm{Se}$ consisting of a $\mathrm{Cd} / \mathrm{Se}$ core with a $\mathrm{ZnS}$ shell, proving a wavelength around 470-655nm [120]. QD's can be made biocompatible with increased water solubility, core durability, and suspension characteristics by adding various surface modifications [121]. While QD's have the ability to function as drug carrier molecules, their small size and quantum-confinement properties have mainly promoted their use as imaging agents.

Currently, applications of QD's in photodynamic therapy have shown promise as a novel treatment method, in which light is used to increase the potency and therapeutic efficacy of the particles. For example, it was shown that UV-B irradiation was the most effective method for increasing the therapeutic efficacy of QD's in A549 cell lines by amplifying both apoptotic and necrotic pathways [122]. Another group functionalized QD's with two antibodies for the detection of micro-metastases of lung cancer using patient blood samples, displaying the capability of detecting lung cancer using peripheral blood. The test was shown to be reliable with a positive detection rate of $81 \%$ ( 21 out of 26 lung cancer samples) and no false positives [123]. Furthermore, recent studies have demonstrated that QD's possess enhanced photostability and imaging capabilities as compared to traditional organic dyes. For example, the influence of paclitaxel on endocytotic trafficking in live A549 cells was studied by labeling QD's with epidermal growth factor (EGF). By analyzing the endocytosis and post-endocytotic trafficking of the EGFR, it was shown that cells treated with cisplatin have shortened endosomal trafficking pathways and a $30 \%$ decrease in directed motion [124]. Related studies have been performed on the use of QD's for the diagnosis of lung cancer [125-129]. Overall, QD's have displayed promise as imaging agents for the 
detection of tumor, yet limitations such as toxicity, specificity, and sensitivity must be further evaluated before clinical application.

Dendrimers are highly branched 3D synthetic polymeric nanoparticles that extend from a center core from which multiple generations of monomers attach. Dendrimers can range in size from 2-20 nm, and their synthesis must be carefully controlled to ensure the addition of one monomer layer at a time [130]. Their synthesis can be completed by two methods: (1) either growth from the inner to outer regions, also known as divergent synthesis, (2) or from the outside inward to the core, known as convergent synthesis [130]. Multiple branches (i.e. monomers) can be added to the core and each set of branches is known as a generation. Dendrimers have many properties that make them suitable nanoparticles for biological purposes including their small size that is similar to that of proteins and DNA, numerous active surface groups capable of bio-conjugation, an interior open space that can be used for drug loading, minimal immunogenicity, and the ability to modify their excretion from the body through size and surface charge alterations [131-133].

Movassaghian et al. developed dendrosomes with a non-cationic liposomal shell containing an antisense oligonucleotide that increased the suppression of the target gene, protein kinase C (PKC)-alpha in A549 cells with high encapsulation efficiency and no visible toxicity [134]. Wang et al. further functionalized dendrimers by synthesizing folic acid-modified dendrimer-entrapped gold nanoparticles for use as CT imaging agents in lung cancer. These novel dendrimers showed enhanced biocompatibility with no alterations on cell morphology or viability, demonstrating the potential use of dendrimers as imaging agents with low toxicity [135]. Dendrimers have also been conjugated to various proteins or peptides in hopes to increase the therapeutic efficacy of the protein or peptide. For example, after discovering a novel NSCLC-targeting peptide known as lung cancer targeting peptide (LCTP), Liu et al. conjugated the peptide to acetylated polyamidoamine (PAMAM) dendrimers. It was concluded that although the dendrimerpeptide conjugate was successfully targeted to the NSCLC tumors, more studies were needed for analyzing its efficacy as a drug carrier molecule [136]. The addition of cisplatin onto dendrimers has been studied by many groups, including Malik et al. who conjugated cisplatin through the sodium carboxylate surface of PAMAM dendrimers. The success of this formulation was studied in B16F10 tumor mice, and further 
application of this formulation could be applied for lung cancer [137]. Additional studies using dendrimers for studying lung cancer have been performed by [138-141].

Liposomes consist of closed spherical assemblies of amphiphilic phospholipid bilayers, capable of encapsulating drugs or other molecules within an inner aqueous core. Liposomes are the most extensively studied type of nanoparticle for the treatment of most cancers due to their ease of synthesis, minimal toxicity, and biodegradability. Many groups have successfully targeted liposomes containing two common anticancer agents used for the treatment of lung cancer, cisplatin or paclitaxel, to lung tissue [142-144]. For example, Zhou et al. loaded paclitaxel onto liposomes with a mitochondrial targeting molecule for the treatment of drug-resistant lung cancer. Using the A549/cDDP xenograft tumor model, targeted paclitaxel liposomes experienced significantly enhanced uptake into the mitochondria of the lung cancer cells, resulting in enhanced apoptosis by acting on the mitochondrial signaling pathways [145]. A similar study by Li et al. developed lonidamine liposomes in combination with epirubicin liposomes for the con-current treatment of drug resistant lung cancer. They showed that lonidamine liposomes in combination with an anticancer agent can enhance the response of drug resistant lung cancer through action on the mitochondrial signaling pathway [146]. Clinically, there are stage III trails analyzing the use of liposomal cisplatin in combination with paclitaxel for a first line treatment of NSCLC with reduced systemic toxicity and increased systemic drug circulation time. There were no significant differences between group (A) liposomal cisplatin in combination with paclitaxel and (B) cisplatin in combination with paclitaxel, yet there was significant decreased nephrotoxicity, leucopenia, neuropathy, nausea, vomiting, and fatigue [147]. There are multiple clinical trials using liposomal forms of cisplatin for the treatment of NSCLC, including Lipoplatin and SPI-77 [148-152].

Doxorubicin is another anticancer drug that has been successfully encapsulated using liposomes that have shown greater efficacy in the treatment of lung cancer compared to freely circulating drug [153156]. Liposomal doxorubicin in combination with liposomal tumor necrosis factor-related apoptosisinducing ligand (TRAIL) was shown to have a stronger antitumor effect when used together, as doxorubicin sensitizes cells to TRAIL-induced apoptosis [157]. Saad et al. designed liposomes for the codelivery of doxorubicin and siRNA targeted to suppressors of pump and non-pump cellular resistance 
mechanisms. The study revealed suppression of cellular resistance by the siRNA and increased therapeutic efficacy due to the doxorubicin [55].

Some groups are analyzing new methods for sensitizing lung cancer cells to current chemotherapeutics such as cisplatin. For example, Ou et al. developed a liposomal form of a novel tumor suppressor gene, LKB1, in combination with low-dose cisplatin to decrease the number of lung metastatic tumor nodules in vivo. The use of siRNA as a therapeutic treatment in lung cancer has recently been studied by multiple groups $[55,158]$, including Taetz et al. who created cationic hyaluronic-modified liposomes for the targeted delivery of anti-telomerase siRNA to CD44 receptor-expressing A549 lung cancer cells [159]. There are many additional groups who have used liposomes for both treatment and diagnostic purposes in lung cancer [156, 160-164].

Polymeric Nanoparticles are colloidal particles of biodegradable polymer matrices that can range in size from 10-1000 nm. Current uses of polymeric nanoparticles in medicine include delivery of plasmic DNA, proteins, peptides, water-insoluble drugs, contrasts agents, and other compounds to targeted tissue. The most commonly used polymers used in lung cancer nanotherapeutics include polylactic acid (PLA) [165-167], poly(lactide-co-glycoside) (PLGA) [168-170], poly(ethylene glycol) (PEG) [171-176], poly(ethyleneimine) (PEI) [177-181], chitosan [182-186], and gelatin [187-190]. Nguyen et al. developed conjugates that coupled cell penetrating peptides (CPP) to PEI, through a PEG linker. Overall, the addition of PEG and coupling of CPPs to PEI enhanced the bioactivity of the particles after intra-tracheal delivery [191]. Similarly, Koshkina et al. analyzed the biodistribution of human p53 plasmid with PEI in vivo through inhalation, which showed increased lung accumulation that lasted for at least 24 hours [192]. While PEI functions as a gene-delivery polymer, its toxic nature makes it unsuitable for clinical applications. To overcome toxicity, Hong et al. developed a novel biocompatible polymer, glycerol triacrylate-spermine (GT-SPE) for the delivery of gene therapy in lung cancer. The particles possessed an average size of 121 $\mathrm{nm}$ with a strong anionic charge, as the particles protected the DNA from nucleases and decreased proliferation and angiogenesis markers in a murine model [193].

Polymeric nanoparticles have been utilized for photodynamic therapy, the process in which a photosensitizer produces singlet oxygen and other reaction oxygen species that lead to peroxidation and ultimately to cellular death. Fadel et al. developed biodegradable copolymer PLGA nanoparticles for the 
delivery of zinc (II) phthalocyanine ( $\mathrm{ZnPc}$ ), a photosensitizer for photodynamic therapy. The PLGA nanoparticles encapsulating $\mathrm{ZnPc}$ showed enhanced tumor regression as compared to freely circulating $\mathrm{ZnPc}$, demonstrating that polymers can assist in delivering nanoparticles to regions of interest [194]. Jung et al. prepared polymeric nanoparticles using polylactic acid and an amphilic block copolymer (mPEGPLA) for the delivery of paclitaxel in A549 xenograft mice. The polymeric nanoparticles exhibited enhanced anticancer and radiotherapeutic efficacy in vivo by intravenous injection of the paclitaxel loaded nanoparticles [195]. The feasibility of delivering biodegradable polymeric nanoparticles via inhalation for drug delivery was studied by Beck-Broichsitter et al. using 5-(6)-carboxyfluorescein entrapped in biocompatible, fast degrading, branched polyester nanoparticles. The nanoparticles were delivered to an isolated rabbit lung model, and displayed rapid release kinetics with $90 \%$ of drug being released during the first fifty minutes along with encapsulation efficiencies $\sim 60 \%$. Overall, their approach showed that these new polymeric nanoparticles were excellent candidates for the pulmonary delivery of nanomedicine through inhalation [196]. Recently, Anselmo et al. attached PLGA nanoparticles to red blood cells (RBC) to decrease their uptake in the liver and spleen resulting in increased tumor accumulation. While the nanoparticles would travel throughout circulation, they would eventually mechanically transfer the nanoparticles from the RBC surface to the lung endothelium [197]. 


\section{$\underline{\text { Inorganic Nanoparticles for Lung Cancer }}$}

Magnetic nanoparticles are biocompatible, non-toxic, chemically stable particles that vary in size from 5-200 nm and possess magnetic properties that can be utilized for the enhancement of therapeutic efficacy. The applications of magnetic nanoparticles in cancer therapy have included thermotherapy, targeted delivery of drugs, magnetic cell separation, and imaging agents (e.g. MRI). The most commonly investigated magnetic nanoparticle is comprised of an iron-oxide core, $\mathrm{Fe}_{3} \mathrm{O}_{4}$. While thermotherapy has shown promise in decreasing tumor size, inadequate intra-tumoral accumulation of nanoparticles often results in sub-lethal temperature increase and inadequate therapeutic response. To enhance the delivery of superparamagnetic iron oxide (SPIO) nanoparticles to NSCLC in vivo tumors, Sadhuka et al. actively targeted the nanoparticles to EGFR resulting in superior intra-tumoral particle accumulation and significant inhibition of the lung tumor growth [198]. Concurrent delivery of multiple drugs on a single nanoparticle provides additional benefit in comparison to single drug-loaded nanoparticles and to freely circulating drug, as most cases of lung cancer will be treated with a regimen of two or more drugs simultaneously. Liu et al. synthesized two types of magnetic mesoporous silica nanoparticles co-loaded with either doxorubicinpaclitaxel or doxorubicin-rapamycin. Using A549 cells, the group showed increased efficacy of the dualdrug loaded nanoparticle in comparison to single drug loaded nanoparticles [46]. To further study the applications of magnetic nanoparticles, Guthi et al. developed multi-functional micelles that entrapped SPIO nanoparticles containing doxorubicin for the concurrent imaging and treatment of lung cancer. The SPIO-loaded nanoparticles were targeted using a lung cancer-targeting peptide, which resulted in a 3 -fold increase of SPIO loaded micelles in comparison to control untargeted micelles, thus showing the potential of "smart" targeted, image-guided treatments for lung cancer [199].

The application of magnetic nanoparticles for purposes such as magnetic hyperthermia has been of interest recently due to the non-invasive approach and efficiency of tumor ablation. The process of magnetic hyperthermia occurs when SPIO nanoparticles are exposed to an alternating magnetic field, causing the nanoparticles to release heat within the cell resulting in cell death. For this method to be efficacious in practice, an adequate number of injected nanoparticles must be able to reach the malignant cancerous cell residing within solid tumors. To overcome this problem, Sadhukha et al. synthesized inhalable SPIO nanoparticles targeted to the EGFR, which resulted in an increase of nanoparticle 
accumulation in the tumor adequate for significant inhibition of lung tumor growth after magnetic hyperthermia [198]. Similar studies have utilized magnetic nanoparticles for the treatment and imaging of lung cancer [200-205].

Metallic Nanoparticles are synthesized from various metals including gold [19, 206-210], silver [211-214], copper [215-217], palladium [218], ruthenium [219], and nickel [220-222]. These nanoparticles have been proven to be multi-functional, as they can be utilized in numerous applications including drug delivery and in vivo imaging, such as photoacoustic imaging. On the contrary, poor biocompatibility and uncertain fate in vivo has hindered their use clinically [223]. Wang et al. showed that gold nanoparticles coated with 1-stearoyl-2-oleoyl-sn-glycero-3-phospho-(1'-rac-glycerol) were readily uptaken into A549 cells, yet they induced the formation of lamellar bodies which could export the nanoparticles out of the cell, thus acting as a cellular resistance mechanism [224]. In Chapter 2, England et al. analyzes the diffusion of three types of citrate gold nanoparticles in 3D cell cultures of A549 cells. Nanoparticles with multiple layers, including PEG, phosphatidylcholine (PC), or PC with high density lipoprotein (HDL) were developed. From analyzing the diffusion patterns of these coated nanoparticles in 3D cell cultures, they show that nanoparticles coated with both PC and HDL exhibited enhanced tissue penetration in comparison to PEGylated or PC-only coated nanoparticles. While PEG is the primary polymer used for enhancing the circulation time of nanoparticles, this study suggests that the multi-layering of gold nanoparticles may augment transport within solid tumors [225]. Some groups have attempted to use gold nanoparticles to diagnose lung cancer using non-invasive approaches. Recently, gold nanoparticles have been shown to be effective sensors in diagnosing lung cancer in exhaled breath by detecting high concentrations of several volatile organic compounds [226] and through histological classification by binding to biomarkers of distinct lung cancer types [227].

As metallic nanoparticles have been associated with possible toxicity concerns, multiple groups are searching for ecofriendly and nontoxic methods for synthesizing nanoparticles. As the manufacturing of nanoparticles can pose health concerns for workers, finding better methods for creating nontoxic nanoparticles is of particular interest. For example, Bhat et al. developed a green chemistry approach to synthesize gold nanoparticles with the mushroom Pleurotus florida through a photo-irradiation method. The bio-functionalized nanoparticles showed enhanced anticancer properties in A549 cells, as well as four 
other cancer cell lines [228]. Many other metals been have studied for their potential for biological purposes, yet most have displayed toxicity in vivo. Nickel nanoparticles are of particular interest due to their use in modern industries for purposes such as sensors and catalysts. A link between nickel nanoparticles and reduced mitochondrial function was recently discovered. In addition, nickel nanoparticles resulted in leakage of lactate dehydrogenase in a dose and time-dependent manner in A549 cells, suggesting the metallic nanoparticles are toxic to human lung epithelial cells [229].

Nanoshells are synthesized using a template-mediated method with an inner core composed of various materials including silica, polystyrene, poly (lactic-co-glycolic) acid, and $\mathrm{Fe}_{3} \mathrm{O}_{4}$ with outer metallic shells. For example, silica-gold nanoshells are composed a dielectric silica core with an outer metallic shell of gold, and have been studied extensively for applications in lung cancer [230, 231]. They are optically tunable for specific applications, as the maximum wavelength and hydrodynamic size can be altered chemically by altering the thickness of the outer metallic shell. Interestingly, nanoshells can be optimized to exhibit maximum absorbance values in the NIR-spectral range, making them candidates for both multimodality imaging and photothermal therapy. The Stöber process is used to synthesize silica gold-nanoshells through functionalization of organosilane molecules such as aminopropyltriethoxysilane (APTES) [232]. After formation of the silica cores, ultra-small gold nanoparticles with diameters $\sim 1 \mathrm{~nm}$ are covalently attached to the core creating a shell of gold around the silica core [233]. After this process, nanoshells can be functionalized using various chemical entities such as polymers, lipids, or targeting ligands. England $e t$ al. functionalized silica gold nanoshells with three different surface entities to compare physiochemical characteristics and tissue diffusivity in A549 3D cell culture. By applying different surface modifications, the size of the nanoshells ranged from $145-160 \mathrm{~nm}$ and the wavelength fluctuated from 820 to $860 \mathrm{~nm}$, showing their highly tunable properties [225].

Using lasers corresponding to the maximum wavelengths of these nanoparticles, photothermal therapy can be used to excite the particles resulting in increased temperatures as the nanoparticles release vibrational energy in the form of heat in the surrounding tissue, promoting cell death. In the process of photothermal therapy, electromagnetic radiation from the laser is absorbed by the nanoparticles and transformed into heat, resulting in irreversible damage to the target tissue and triggering cell death [234]. The uniqueness of this treatment is its minimal invasiveness, as laser light can travel through skin tissue 
with minimal transfer of energy. While most photothermal therapy has been used with subcutaneous tumors, Bagley et al. recently showed the feasibility of photothermal therapy for intraperitoneal tumors by using small implanted NIR sources, which may also have implications for lung cancer [235].

Previous studies have shown that nanoshells are more effective at photothermal therapy than other types of theranostic nanoparticles such as gold nanorods. For example Cheng et al. showed that silica-gold nanoshells that underwent laser activated photothermal therapy experienced an increase in temperature of the environment by $25^{\circ} \mathrm{C}$, while gold nanorods only exhibited a $10^{\circ} \mathrm{C}$ increase in temperature at 7 minutes of laser irradiation $\left(30 \mathrm{~V} \mathrm{~cm}^{-2}\right)$. From this study, it was concluded that silica-gold nanoshells required the lower nanoparticle accumulation in the tissue to induce cell damage, thus making them optimal candidates for future studies [230, 236]. While photothermal therapy is currently not available for human use, some groups have recently shown its feasibility [237]. Liu et al. used gold nanoshells with carboxylated polystyrene spheres for photothermal therapy in Lewis lung carcinoma mice, which resulted in an average tumor inhibition rate of over 55\% in comparison to controls [231]. Clinical trials using silica-gold nanoshells for photothermal therapy have only recently begun in cancers such as melanoma and head-andneck cancers. There is currently an ongoing clinical trial using gold nanoshells for photothermal therapy in human head and neck cancer (Nanospectral Biosciences, Inc.) [238], and it has recently been declared that the first clinical trial for gold nanoshells in the treatment of lung cancer will be carried out in the United States [239].

Ceramic Nanoparticles are synthesized from ceramic chemicals such as hydroxyapatitie [240], silica [241-244], nitrides (e.g. boron nitride) [245], and oxides (e.g. zinc, titanium, iron oxide, manganese) [246-250]. Currently, hollow mesoporous nanospheres are of particular interest due to their high drug loading efficiency and minimal toxicity in vivo. Drugs such as bortezomib, the first clinically approved proteasome inhibitor, experienced limited efficacy due to its poor water-solubility and stability. Using hollow mesoporous silica nanospheres containing bortezomib, Shen et al. showed improved anti-tumor efficacy in comparison to drug alone in an NSCLC cell line with a 42\% decrease in IC50 from the bortezomib- loaded nanoparticles [242]. Other groups have also shown effective drug loading capabilities, including Huang et al. who demonstrated the potential use of mesoporous nanoparticles are imaging agents for MRI and pH-controlled drug release [243]. Ashokan et al. also studied the applications of ceramic 
nanoparticles for imaging purposes (MRI, X-ray, NIR fluorescence) by using hydroxyapatite nanocrystals, which exhibited selective accumulation in cancer cells through targeting of the folate receptor [240].

EGFR targeting has been of great interest due to its overexpression in most cancer cell lines [251253]. Sundarraj et al. recently targeted mesoporous silica nanoparticles to EGFR overexpressing NSCLC cells, with an anticancer agent pryyolidine-2. Using EGFR as the targeting agents, the nanoparticles showed enhanced accumulation in the NSCLC cells with enhanced therapeutic efficacy [254]. Some types of nanoparticles can be combined with the goal of enhancing their therapeutic efficacy in vivo, such as polymeric micelles and mesoporous silica nanoparticles. For example, Yuan et al. studied cellular uptake and cytotoxicity of doxorubicin-loaded stearic acid-g-chitosan nanoparticles in A549 cells demonstrating faster cellular uptake with increased silica ratio [255]. Despite the advantages of ceramic nanoparticles in drug delivery and cancer therapy, questions have been raised about the potential toxic effects. Another type of ceramic nanoparticle, titanium dioxide, has been shown to induce genotoxicity and apoptosis in A549 cells [256, 257]. To explore the potential toxicity of silica nanoparticles, Ahamed investigated the induction of cytotoxicity, oxidative stress, and apoptosis caused by silica gold nanoparticles in NSCLC, showing a dose-dependent cytotoxicity in A549 cells, along with induction of oxidative stress in a dose-dependent manner [244]. 


\section{$\underline{\text { Nanotoxicology }}$}

Administration of nanoparticles to the lungs can be accomplished through systemic circulation (e.g. intravenous injection) or pulmonary inhalation. Systemic circulation can result in systemic toxicity as the nanoparticles distribute within other tissues and organ systems, while inhalation provides a direct route to the lungs [258]. Both intentional administration of nanoparticles for medicinal purposes and occupational exposure as a result of inhaling aerosols released during the production process of various nano-materials can result in toxicity. As there are no universal parameter sets required for synthesis and study of nanoparticles, it is difficult to directly compare the associated toxicity of nanoparticles. Recent reports suggests that certain characterization processes should be completed for each type of nanoparticle synthesized, including information regarding size distribution, shape, composition, agglomeration status, dissolution, purity, surface area, and surface modifications [259]. If each type of nanoparticle synthesized were characterized by a set of standards, direct comparisons of toxicity could be made providing enhanced insight into treatments for nanoparticle overdose and accidental exposure.

Numerous studies have analyzed the toxicities associated with both systemically distributed and inhalable nanoparticle therapies [260-266]. Park et al. studied the toxicity of various inhalable metallic nanoparticles (i.e. titanium oxide, gold, alumina, zinc, and nickel) by analyzing the apoptotic and morphological damages caused by the nanoparticles in A549 cells. They showed a dose-dependent toxicity with zinc nanoparticles having the most apoptotic effect and titanium oxide nanoparticles exhibiting the most morphological damage [267]. Another study further analyzed the toxic effects of metallic oxide nanoparticles in comparison to multi-walled carbon nanotubes (MWCNT). Results from this study showed that copper oxide nanoparticles displayed the most toxicity regarding cytotoxicity and DNA damage. In the study, zinc oxide nanoparticles showed significant adverse effects on cell viability, yet iron oxide nanoparticles showed minimal toxicity [268].

Nanoparticles delivered via inhalation will distribute throughout the airways with size-dependent deposition patterns, with particles $<10 \mathrm{~nm}$ depositing primarily in the tracheobronchial regions, particles 20-30 nm depositing in the alveolar regions, and particles 30-100 nm depositing within all regions of the lung [269]. Currently, there is a lack of research analyzing the potential toxic effects of nanoparticles in 
human lungs, although some groups have studied the deposition patterns of inhaled nanoparticles using computational models [270-272].

The most common toxicity associated with the use of inhaled nanoparticles in vivo is pulmonary fibrosis, or an increased amount of collagen within the lung tissue. Currently, carbon nanotubes have been the most common nanoparticle studied in reference to potential toxicity. Mitchell et al. analyzed the toxicity of inhaled MWCNT, showing that they did not result in significant lung inflammation or tissue damage after 7 days (6 hour exposure/day), yet higher concentrations of the MWCNTs did cause nonmonotonic system immunosuppression after 14 days, which was primarily characterized by a reduction in T-cell dependent antibody as well as T-cell proliferation [273]. Other studies have identified nanoparticles as potential toxic entities through in vitro cytotoxicity testing. For example, Vandebriel and De Jong recently summarized the mammalian cellular toxicity of zinc oxide nanoparticles by showing that their primary toxicity was due to low solubility of nanoparticles and the decreased contractility of airway smooth muscle in the lungs. Other common toxicities were also evaluated including cytotoxicity, oxidative stress, intracellular calcium flux, decreased mitochondrial membrane potential, and numerous immune reactions [274].

While all nanoparticles exhibit some degree of toxicity in a dose-dependent manner, metallic nanoparticles have shown increased risk as their structure is prone to agglomeration. As more people are exposed to metallic nanoparticles in industrial plants, there is a growing concern about the potential systemic toxicities associated with chronic exposure to metallic nanoparticles. Yu et al. studied the effects of metallic nanoparticles in vivo, where rats were treated with $2 \times 10^{6}$ nanoparticles per $\mathrm{cm}^{3}$ by inhalation for 6 hours per day for 15 days. Exposure for five days resulted in accumulation of gold within the lungs and by day 15 , nanoparticles were detected in the esophagus, tongue, kidneys, aorta, spleen, heart, and blood. There was also down-regulation of genes related to muscle function in the lungs, displaying potential lung toxicity [275]. The location of nanoparticle accumulation in vivo can directly correlate with toxicity, as certain organs, such as the liver and spleen, will accumulate more nanoparticles than other organ systems. Toxicity testing focuses on these particular sites [276]. Additional studies have been performed analyzing possible links between nanoparticle size and toxicity. For example, Chen et al. showed that gold nanoparticles ranging in size from 8-37 $\mathrm{nm}$ caused severe sickness in mice that received repeated 
intraparental injections [277]. Ferin et al. studied the retention of ultrafine nanoparticles inhaled by rats exposed for 12 weeks resulting in acute inflammatory response in the lungs [278]. In addition, Geys et al. studied the dose-dependent toxicity of nanoparticles using carboxyl-coated quantum dots that caused vascular thrombosis [279].

Overall, several factors must be considered when analyzing the toxicity of nanoparticles. First, while nanoparticles may display toxicity in cell lines (i.e. in vitro), one should recognize that toxicity in vitro does not always correlate with toxicity in vivo as the animal model is more complex than cell cultures. For example, while fullerenes were highly toxic in vitro, the in vivo finding showed no adverse effects in the lung tissue at 3 months post-exposure to the highest concentration of fullerenes available [280]. In another study, Sayes et al. tested five different nanoparticles for toxicities including carbonyl iron, crystalline silica, amorphous silica, and zinc oxide nanoparticles. It was demonstrated that toxicity between in vitro and in vivo should not be correlated without experimental results, as nanoparticles showing toxicity in vitro did not show toxicity in vivo and vice versa [281]. Another factor that can influence the toxicity profile of nanoparticles is surface modifications. Even simple surface alterations or modifications can result in significant changes in toxicity. This supports the need for a set of characterization standards by which nanoparticles can be assessed. In conclusion, the toxicity of nanoparticles is highly dependent upon the type of particle being employed and should not be estimated based upon in vitro results alone. While in vitro toxicity may indicate the potential for in vivo toxicity, little correlation has been established between cell culture and animal models. Additional studies are needed to determine the possible toxicities associated with nanoparticle usage and to determine optimal treatment strategies. 


\section{Hypotheses and Aims}

\section{Nanotherapeutics provide an alternative method for delivering chemotherapeutics to solid}

tumors, yet the transport of nanoparticles in vivo is not fully understood. After systemic injection of nanoparticles, a large portion of injected dose will become sequestered by macrophages of the liver and spleen resulting in a diminished treatment. Once nanoparticles localize the tumor, they must extravasate from the irregular vasculature and diffuse throughout the tissue reaching all malignant cells. Currently, there is little research regarding the movement of nanoparticles throughout tumor tissue.

We will examine the potential use of two and three layer gold nanoparticles for treating cancerous solid tumors by investigating their diffusivity properties, drug loading and release capabilities, and their efficiency at causing cell death. We hypothesize that smaller nanoparticles with enhanced diffusivity properties, such as colloidal gold nanoparticles, will be more efficacious in the treatment of solid tumors.

Aim 1: Design two and three layer gold nanoparticles and examine the diffusivity of these particles in 3D cell cultures. Hypothesis: Two and three layer gold nanoparticles will diffuse further than previously developed PEGylated nanoparticles because the physiochemical properties of the layered nanoparticles.

a) Synthesize two and three layer colloidal gold nanoparticles and silica gold nanoshells and characterize particles to ensure presence of surface moieties.

b) Determine the accumulation of nanoparticles in 3D cell cultures of human lung, pancreas, and liver cell lines.

c) Compare the diffusivity of two and three layer nanoparticles to the diffusivity of previously developed PEGylated nanoparticles in 3D cell cultures.

Aim 2: Examine the biodistribution and diffusivity of PC-coated two layer citrate gold nanoparticles and silica gold nanoshells in vivo. Hypothesis: We hypothesize that size-dependent localization of nanoparticles will occur, with smaller citrate gold nanoparticles accumulating at higher concentrations and diffusing further from vasculature in the orthotopic pancreatic tumor in comparison to the larger silica gold nanoshells, as they have been shown to be hindered due to their large size. 
a) Compare the accumulation of two layer colloidal gold nanoparticles and silica gold nanoshells in the liver, spleen, and pancreatic tumor.

b) Analyze the diffusivity of two layer citrate gold nanoparticles and silica gold nanoshells from nearest extravasation sites in liver, spleen, and pancreatic tumor sections.

Aim 3: Analyze the drug loading capabilities and release kinetics of two and three layer colloidal gold nanoparticles. Hypothesis: We hypothesize the hydrophobic region between the first and second layer of the nanoparticles is optimal for loading of hydrophobic compounds, including paclitaxel. For this reason, the loading efficiency of paclitaxel will be high with a controlled release pattern.

a) Synthesize two and three layer colloidal gold nanoparticles loaded with cisplatin or paclitaxel.

b) Determine the loading efficiency of paclitaxel and cisplatin onto each type of particle.

c) Analyze the drug release patterns to determine the best model to describe the release of drug from each type of nanoparticle.

Aim 4: Compare the efficacy of two and three layer nanoparticles loaded with cisplatin or paclitaxel to free drug using 3D cell culture models of lung cancer. Hypothesis: We hypothesize that drug loaded nanoparticles will show similar efficacy at causing cell death as free drug because layered nanoparticles release drug while diffusing throughout tissue.

a) Examine the efficacy of free cisplatin and paclitaxel in monolayer cell cultures.

b) Compare the drug dosages required for cell death in monolayer cell cultures and 3D cell cultures.

c) Analyze the efficacy of cisplatin or paclitaxel-loaded two and three layer nanoparticles in 3D cell cultures. 


\section{CHAPTER II}

\section{ENHANCED PENETRATION INTO 3D CELL CULTURES USING TWO- AND THREE LAYERED NANOPARTICLES}

\section{$\underline{\text { Background }}$}

Microenvironment of Solid Tumors

In addition to excessive cellular proliferation, solid tumors typically elicit irregular angiogenesis resulting in structurally abnormal and leaky vascular structures. The passive mechanism of nanoparticle accumulation (EPR effect) takes advantage of this situation by enabling systemically administered nanoparticles sized $10-400 \mathrm{~nm}$ to preferentially extravasate from the vasculature into the interstitial space of solid tumors [282, 283]. Additional factors contributing to nanoparticle pharmacodynamics and cytotoxicity include size, surface charge and morphology [284, 285]. These properties are typically tailored to design systems that exhibit optimal tumor tissue uptake. Drugs delivered via nanoparticles can thus increase treatment effectiveness while reducing systemic toxicity.

In particular, gold nanoparticles have shown promise in cancer treatment. Bulk gold itself is an inert material, though nanoparticles smaller than $5 \mathrm{~nm}$ in diameter have documented cytotoxic properties [286, 287]. Larger particles have demonstrated little cytotoxicity, depending on the surface charge. Cationic particles can be more cytotoxic at lower concentrations compared to anionic nanoparticles, which has been attributed to electrostatic interactions of the positively-charged particles with negatively-charged cell membranes [288]. Surface coatings of poly-(ethylene glycol) (PEG) can effectively hide the nanoparticles from the immune system, further lowering systemic toxicity [284, 287].

However, heterogeneity in blood flow due to irregular angiogenesis and vascular remodeling at the tumor site promote tissue hypoxia and thus cell quiescence, presenting a physical barrier to cell-cycle dependent chemotherapeutics delivered by nanocarriers [289-291]. Inadequate vascularization leads to 
further impediments that hinder optimal treatment, including insufficient drug dosages due to abnormally long inter-vascular diffusion distances, as well as disturbed convection and diffusion of molecules (such as glucose, oxygen) and nanoparticles in the interstitium [289, 292-294], as has recently been explored through intravital microscopy and mathematical modeling [292, 295]. Cancerous tissue can contain almost twice the volume of interstitial space compared to normal tissue [289, 294]; an abundance of ECM proteins along with an increased interstitial pressure may further inhibit nanoparticle delivery and drug diffusion in under-vascularized tumor regions [289, 294, 296]. These physical barriers, coupled with intrinsic resistance mechanisms at the cell-scale, often cause cancer drug therapies to fail. Recently, engineering and physicsbased approaches are being applied to help tackle these challenges in cancer treatment [297].

Estimates of nanoparticle distribution in 3D tissue have been previously obtained in collagen gels [298] as well as in 3D cell culture [24, 299]. Gold nanoparticle accumulation was shown to be size dependent, suggesting that particles $\sim 20 \mathrm{~nm}$ or smaller had superior penetration abilities compared to larger particles [299]. For example, particles < $10 \mathrm{~nm}$ were able to better penetrate breast cancer tumor spheroids representing avascular tumor tissue than larger particles, which has also been confirmed in vivo [24]. It has been shown that while larger polymeric micelles distribute within hypervascularized tumors, only sub-100 $\mathrm{nm}$ particles were able to meaningfully penetrate hypoxic tumors [300]. However, smaller particles $(<50$ $\mathrm{nm})$ are not as useful for drug delivery compared to larger nanoparticles, since they are typically unaffected by the EPR effect and are thus less targeted [24]. Previous studies have shown cellular uptake of both smaller citrate gold nanoparticles and larger silica gold nanoshells, with enhanced cellular uptake of the smaller particles [301-303].

\section{Purpose of Study}

In this study we examine the uptake of functionalized silica gold nanoshells into a 3D cell culture (spheroid) model as a first step to represent avascular liver, lung, and pancreatic tumor tissue. In addition to the nanoshells, citrate-gold nanoparticles were investigated for comparison. Cells aggregate in 3D culture to create avascular nodules with the production of ECM components and the establishment of diffusion gradients of oxygen, glucose, and nanoparticles [290]. Experiments with 3D cell cultures have previously helped to analyze the effect of under-vascularization on particle and drug delivery [299, 304]. Here, particle surfaces were functionalized by a process consisting of either two- or three-layers to optimize uptake into 
tumor tissue. Two-layer particles were synthesized with an inner layer of alkanethiol and an outer layer of PC, a type of phospholipid that is a major component of biological membranes [305]. In order to further optimize tumor uptake, active targeting was promoted by adding a layer of HDL, since the HDL receptor is mainly expressed by liver and cancer cells, thus creating a three-layered particle. Size, zeta potential, and morphology were optimized, and the uptake and distribution were compared to the performance of PEGylated nanoparticles. 


\section{$\underline{\text { Methods }}$}

\section{Synthesis of Citrate Gold Nanoparticles}

Particles were synthesized using the method in which gold chloroauric acid is reduced by trisodium citrate [306]. In this process, 2.2-2.4 mL 1\% wt/v citrate (Fischer Scientific) and $200 \mathrm{~mL} 0.01 \%$ wt/v $\mathrm{HAuCl}_{4}$ (Alfa Aesar, MA) are mixed and heated to boiling, which promotes the reaction of sodium citrate to citric acid. Once the reaction is completed, the solution is concentrated using a rotovapor (Buchi Rotovapor System) to $\sim 20 \mathrm{~mL}$ before the addition of layering to the particles.

\section{Synthesis of Silica Gold Nanoshells}

Particles have an inner core composed of silica with an outer coating of gold. Synthesis follows the Stöber method [232, 307, 308], which consists of four stages: production of small gold seeds, fabrication of monodispersed silica cores, attachment of gold to the seeds, and gold shell growth. The gold colloid solution is created using the recipe from Duff et al [309]. Growth of the silica cores requires the combination of $7.5 \mathrm{~mL}$ tetraethyl-orthosilicate (Sigma Aldrich), $225 \mathrm{~mL}$ absolute ethanol (Decon Labs), and 12.5-13.5 mL ammonia (Sigma Aldrich) [310]. Ammonia is adjusted to achieve silica core sizes 110 +/-5 nm. After removal of the paraffin cover and evaporation of the ammonia, the cores are coated with 3 4\% aminopropyltriethoxysilane (APTES) (Sigma Aldrich). Once the seeds are washed, the 10\% gold solution (THPC) is added. After reaction time, the seeds are washed and redispered in DI water. The seeds are diluted to 0.3-0.5 OD (optical density) at $530 \mathrm{~nm}$ (Varian Cary 50Bio UV-Visible Spectrometer). A sweep of the seeds is performed to optimize the chemical ratio between them, $\mathrm{K}_{2} \mathrm{CO}_{3}-\mathrm{HAuCl}_{4}$, and formaldehyde (Fisher Scientific).

\section{Particle Functionalization}

The first layer applied to the citrate gold particles was Hexadecathiol (Sigma Aldrich) dissolved in ethanol [311]. While stirring, $20 \mathrm{~mL}$ pure ethanol (Decon Labs) was placed in a breaker with $60 \mu \mathrm{L} \mathrm{1-}$ Hexadecathiol being added secondly. The nanoparticles were added to the sample slowly over the next few minutes. The sample was first agitated for 20 minutes, then placed for 12 hours on an orbital rocker (Boekel Scientific). The sample was spun down and the pellet was washed twice and resuspended in chloroform (Sigma Aldrich). The second functionalization was the addition of the PC. The stock solution was made by diluting PC in chloroform, and $100 \mu \mathrm{L}$ were added to the particles after the thiol layer and 
allowed to set overnight on an orbital rocker. The solutions were transferred to glass tubes and the chloroform evaporated at ambient temperature. This process completed the two-layered particles, referred to as $\mathrm{C}_{\mathrm{AU}} / \mathrm{TL} / \mathrm{PC}$ (citrate gold/thiol/phosphatidylcholine) and NS/TL/PC

(nanoshell/thiol/phosphatidylcholine). The three-layered particles, referred to as $\mathrm{C}_{\mathrm{AU}} / \mathrm{TL} / \mathrm{PC} / \mathrm{LP}$ and NS/TL/PC/LP, were created by optimizing the ratio of high-density-lipoprotein (HDL) to nanoparticle optical density, and allowed to react overnight.

PEGylation is considered a common form of surface modification for delivering nanoparticles into cancerous tissue, thus we created PEGylated nanoparticles $\left(\mathrm{C}_{\mathrm{AU}} / \mathrm{PEG}\right.$, citrate gold/pegylated, and NS/PEG, gold nanoshell/pegylated) to compare the efficacy of the synthesized two- and three layer nanoparticles. To prepare PEGylated nanoparticles, 2000 MW PEG was added at a molar ratio of 2500:1. The solution was allowed to react overnight on the rocker. Excess PEG was removed by centrifuging the particles at 13,000G for 20 minutes. The pellet was resuspended in PBS. It is well documented that pegylated particles will stay in circulation longer than non-pegylated particles [312]. To create the pegylated particles, both citrate gold nanoparticles and silica gold nanoshells were incubated with $2000 \mathrm{MW}$ poly(ethylene)glycol overnight and then centrifuged to separate the particles.

\section{Particle Characterization}

Nanoparticle identity was verified as follows. (i) Maximum absorption wavelengths were obtained using the Varian Cary 50 Bio UV-Visible Spectrometer. (ii) Size and zeta potential measurements were obtained using the ZetaSizer Nanoseries ZS90 (Malvera Instruments). (iii) Shape and size were determined using a Zeiss Supra 35VP scanning electron microscope (SEM). (iv) Presence of lipids on the particle cores was confirmed using a Fourier transform infrared (FTIR) instrument (Perkin Elmer Spectrum BX) and through visual analysis using the SEM.

\section{Cell Culture and Particle Experiments}

Human lung adenocarcinoma (A-549) and liver hepatocellular carcinoma (HEPG2) cells were maintained in Dulbecco's modified Eagle's medium (Sigma) with 10\% fetal bovine serum and 1\% penicillin-streptomycin-glutamine solution in a humidified atmosphere of $5 \% \mathrm{CO}_{2}$ at $37^{\circ} \mathrm{C}$. Pancreatic adenocarcinoma (S2VP10) cells were maintained with RPMI 1640 medium (Cellgro) in similar conditions. All cells were grown to $80 \%$ confluence before 3D cell culture formation. Using 24 well ultra-low 
attachment plates (Corning), 100K cells were placed in each well and lightly shaken for $\sim 15$ min. Tumor spheroid formation occurred by self-aggregation after 7-14 days of incubation. Spheroids typically measured between 0.5 and $2 \mathrm{~mm}$ in diameter, though the S2VP10 cells produced smaller, grape-like structures. Spheroids were incubated with $40 \mu \mathrm{L}$ particles at 25 OD for 6 hours before being washed with PBS.

3D Cell cultures were placed in cryomolds made of tissue freezing medium. Samples were allowed to set for two hours, and then sectioned at 5 $\mu \mathrm{m}$ using a Leica CM1860 Cryostat. Sectioned spheroids were then fixed onto Superfrost Plus microscope slides (Fisher Scientific). Successful fixation was determined via bright-field microscopy. Excess media was removed by soaking with $30 \%$ Neutral Buffered Formalin for $1 \mathrm{~min}$. Slides were washed in DI water prior to analysis. All experiments were done in triplicate.

\section{Silver Enhancement Stain}

Slides were placed in cold acetone (Fischer Scientific) for $30 \mathrm{sec}$., then allowed to dry for $1 \mathrm{~min}$. Slides were placed in $10 \%$ formalin buffer (Sigma Aldrich) for 3 min., then washed 2x with DI water and allowed to dry for $5 \mathrm{~min}$. The silver enhancement solution was prepared by mixing $1 \mathrm{~mL} / \mathrm{slide}$ Silver Enhancement Stain A along with $1 \mathrm{~mL} /$ slide Silver Enhancement Stain B (Sigma Aldrich). The two solutions were mixed in a $50 \mathrm{~mL}$ tube and vortexed for $10-15 \mathrm{sec}$. The combined solution $(2 \mathrm{~mL})$ was added to each slide and allowed to react for $6 \mathrm{~min}$. After the reaction time, the solution was washed off $2 x$ using DI water. Slides were analyzed using NIS Elements AR and an Accuscope 3032 inverted light microscope. ROI intensity measurements were recorded for both stained and unstained samples.

\section{Particle Detection using Hyperspectral Imaging}

The CytoViva Hyperspectral Imaging System was used to located nanoparticles within sections of 3D cell cultures. A DAGE camera with the dark field microscope was used to obtain images highlighting the nanoparticles. Particle presence was confirmed using hyperspectral imaging, with data analysis performed using ENVI 4.8 and ImageJ. Using the software, linear [0-255] enhancement was applied and the z-profile library was used to detect single nanoparticles within $3 \mathrm{D}$ cell cultures. Regions of interest (ROIs) were determined and particles within these regions were counted to determine concentration in various tumor areas. The number of particles decreased from the periphery towards the center; accordingly, 
measurements from 10 to $50 \mu \mathrm{m}$ from the tumor periphery were taken to determine spatial distribution of particles into the tumor spheroids.

\section{Statistical Analysis}

Analyses used the two-tailed Student's $t$ test with significant values being less than 0.05 . Statistically significant differences are illustrated with an $(*)$ in the Results. 


\section{$\underline{\text { Results }}$}

\section{Nanoparticle Characterization}

Silica gold nanoshell and citrate gold particles were layered with either two- or three- layers (Figure 4) to promote enhanced penetration into 3D cell cultures of various cancer cell lines, while comparing their penetration and diffusion capabilities to previously designed PEGylated nanoparticles. Nanoparticles were characterized with UV-Visible (UV-Vis) absorbance spectroscopy, zeta potential analysis, dynamic light scattering (DLS), SEM, and FTIR to ensure proper particle formation and modification.

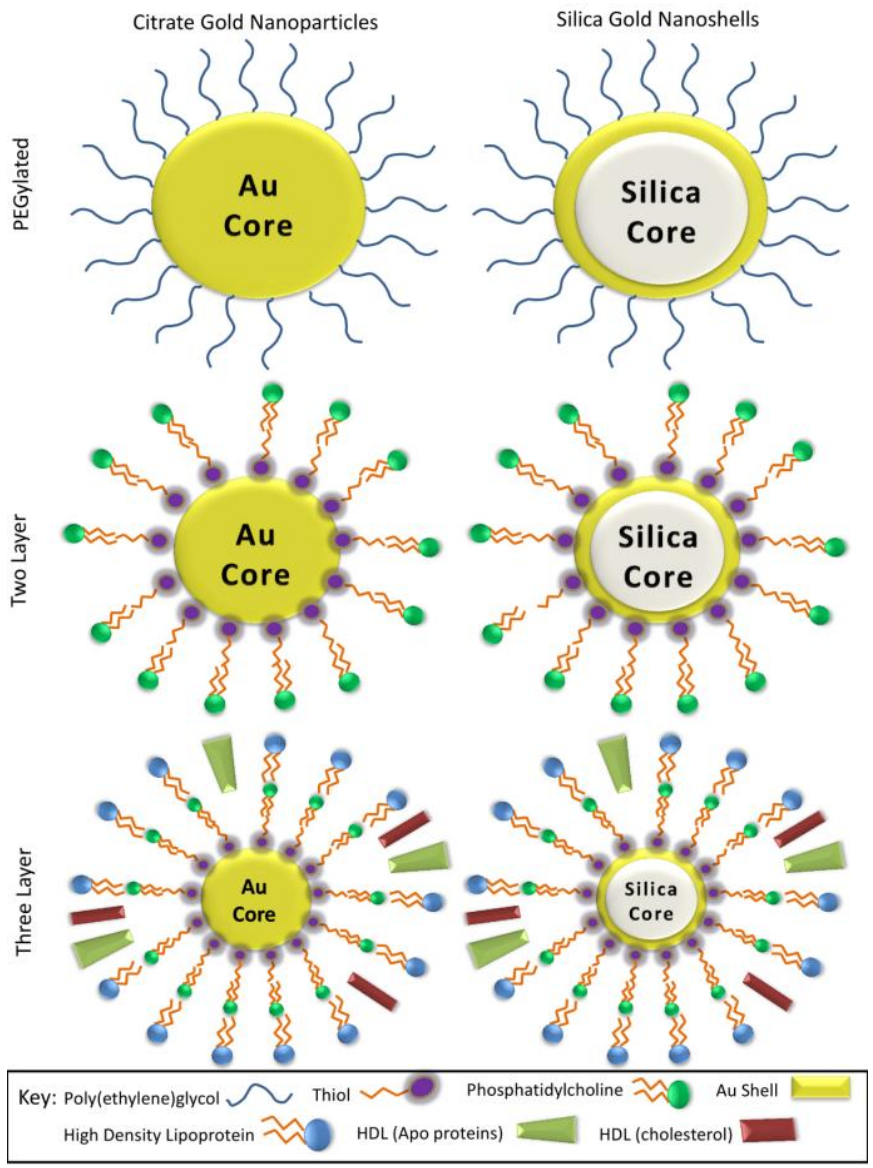

Figure 4. Illustration of Functionalized Gold Nanoshells. Six types of nanoparticles were developed including citrate gold particles and silica gold nanoshells. Particles were modified to form PEGylated, two layer, and three layer particles. 
Optical and charge measurements (Figure 5) indicated that the surface modifications were successful. Prepared citrate-gold nanoparticles (Figure 5A) had a peak absorbance between 533-541 nm (Table 1), which is consistent with previous work [313]. Silica-gold nanoshells (Figure 5B) exhibited peak absorbance between 820-860 $\mathrm{nm}$ (Table 1), which is typical for nanoshells having $110 \mathrm{~nm}$ diameter silica cores and a $\sim 15 \mathrm{~nm}$ gold coating [314]. The spectra displayed minute amounts of noise at higher wavelengths, which is expected for silica gold nanoshells [315]. Nanoshells demonstrated ideal optical properties for near-infrared (NIR) application [316, 317], though such experiments were not included in the scope of this work.

\section{A UV-Vis Spectrum Citrate Gold}

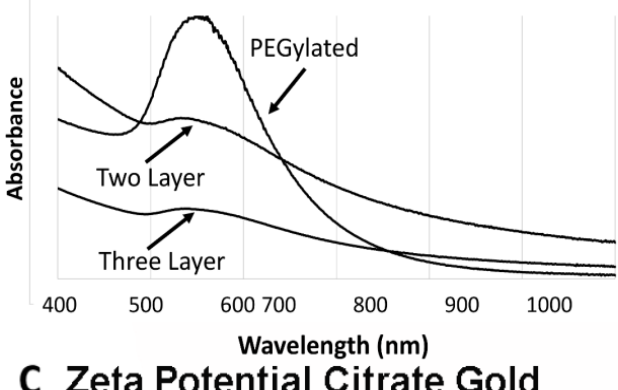

C Zeta Potential Citrate Gold

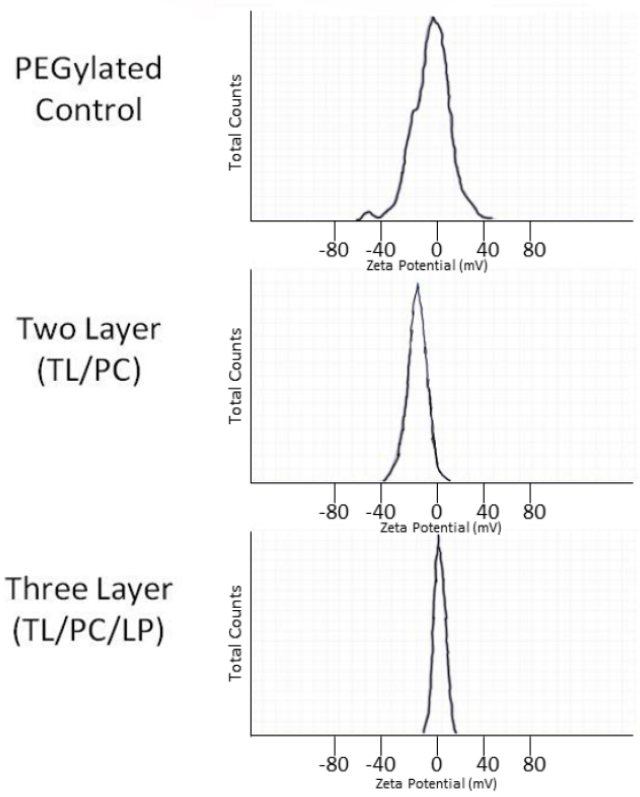

B UV-Vis Spectrum Silica Gold

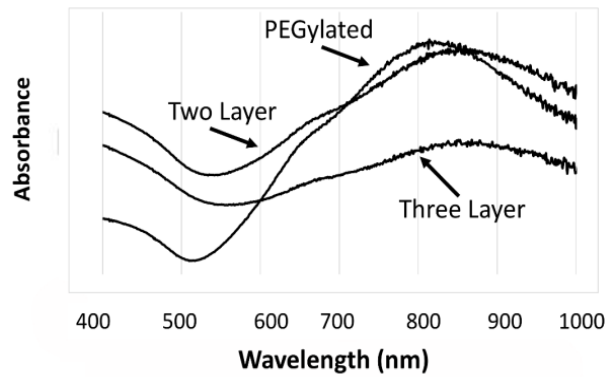

D Zeta Potential Silica Gold
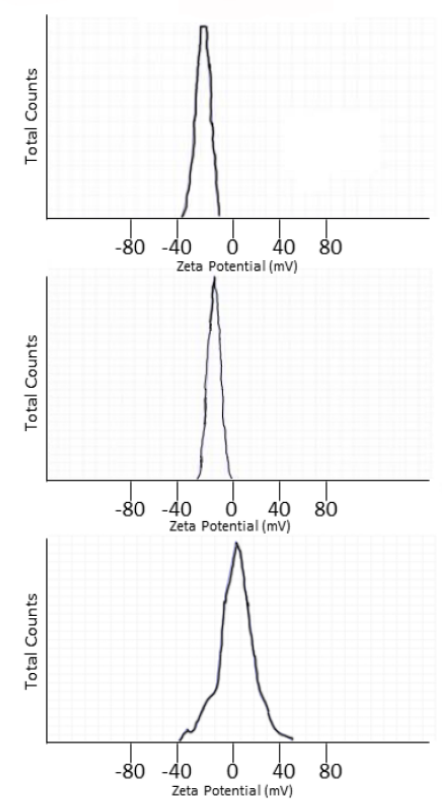

Figure 5. Characterization of Gold Nanoparticles by UV-Visible Spectrometry and Zeta Potential Analysis. Wavelengths for each particle are presented with PEGylated particles (dotted line), two layer (dashed and 
dotted line), and three layer (dashed line). (A) Spectrum of citrate gold nanoparticles indicates a maximum absorption ranging from $\sim 533-541 \mathrm{~nm}$. (B) Spectrum of silica gold nanoshells suggests a maximum absorption at $\sim 820-860 \mathrm{~nm}$. For verification of surface modifications, zeta potential analysis is shown below the spectra as an indication of surface charge. (C) PEGylated citrate gold nanoparticles had a zeta potential of $-9 \mathrm{mV}$, two layer citrate gold nanoparticles had $-20 \mathrm{mV}$, and three layer citrate gold nanoparticles had -2 mV. (D) A zeta potential of -18 mV was found for PEGylated silica gold nanoshells, for two layer silica gold nanoshells it was $-29 \mathrm{mV}$, and for three layer nanoshells it was $-6.8 \mathrm{mV}$.

\section{Table 1}

Determination of Nanoparticle Size and Wavelength

\begin{tabular}{|c|c|c|c|c|c|c|}
\hline & \multicolumn{3}{|c|}{ Citrate Gold Nanoparticles } & \multicolumn{3}{|c|}{ Silica-Gold Nanoshells } \\
\hline & SEM (nm) & $\mathrm{DLS}(\mathrm{nm})$ & $\begin{array}{l}\text { Max Wavelength } \\
(\mathrm{nm})\end{array}$ & SEM (nm) & $\mathrm{DLS}(\mathrm{nm})$ & $\begin{array}{c}\text { Max Wavelength } \\
(\mathrm{nm})\end{array}$ \\
\hline PEGylated (PEG) & $52.6(15.4)$ & $82.81(13.4)$ & 533 & $146.2(9.1)$ & $161.82(12.2)$ & 820 \\
\hline Two Layer (TL/PC) & $47.1(12.6)$ & $74.91(13.3)$ & 540 & $144.9(6.5)$ & $155.11(13.9)$ & 855 \\
\hline Three Layer (TL/PC/LP) & $62.8(14.9)$ & $85.26(18.7)$ & 541 & $158.1(4.6)$ & $169.41(11.8)$ & 860 \\
\hline
\end{tabular}

\section{Nanoparticle Penetration in 3D Cell Cultures}

In order to verify particle surface modifications, the zeta potential was measured (Figure 5C). Modification with PC and thiol resulted in more highly negatively charged particles compared to PEGylated particles. Conversely, HDL caused the zeta potential to become more positively charged when compared to PEGylated particles, but still yields slightly negatively charged particles. PEGylated citrate gold nanoparticles exhibited a zeta potential of $-9 \mathrm{mV}$, while the two-layer citrate gold nanoparticles had a zeta potential of $-20 \mathrm{mV}$ and the three-layer citrate gold nanoparticles had $-2 \mathrm{mV}$. Gold nanoshells generally exhibited higher surface charge than citrate gold nanoparticles, which is consistent with the larger size of the nanoshells. PEGylated nanoshells had a zeta potential of $-18 \mathrm{mV}$, two-layer nanoshells measured a zeta potential of $-29 \mathrm{mV}$, and three-layer nanoshells had a zeta potential of $-6.8 \mathrm{mV}$. These results were in agreement with previous studies [318-320]. 
To ensure the presence of surface modifications, FTIR was performed (Figure 6). PEGylated nanoparticles exhibited C-O-C stretch of the PEG ether $\sim 1050 \mathrm{~cm}^{-1}$ and vibration $\sim 1350 \mathrm{~cm}^{-1}$, along with $\mathrm{CH}_{2}$ and $-\mathrm{CH}_{3}$ bending vibrations around $\sim 1450 \mathrm{~cm}^{-1}$. This stretch is smaller than previously reported [321], possibly due to the addition of PEG onto the silica-core nanoshells. Two layer nanoparticles with an outer layer of PC exhibited $\mathrm{PO}_{4}{ }^{3-}$ group vibrations between $\sim 820-1000 \mathrm{~cm}^{-1}$, C-O-C stretch $\sim 1100 \mathrm{~cm}^{-1}$, with the largest band corresponding to the asymmetric and symmetric $-\mathrm{CH}_{2}\left(2880 \mathrm{~cm}^{-1}\right)$ and $-\mathrm{CH}_{3}(2950$ $\mathrm{cm}^{-1}$ ) stretch and vibration, respectively. For HDL coated nanoparticles, the asymmetric and symmetric $\mathrm{CH}_{2}\left(2880 \mathrm{~cm}^{-1}\right)$ and $-\mathrm{CH}_{3}\left(2950 \mathrm{~cm}^{-1}\right)$ stretch and vibration are still shown along with $\mathrm{C}=\mathrm{O}$ from the lipid ester $\sim 1750 \mathrm{~cm}^{-1}$, amide bonds between $1600-1700 \mathrm{~cm}^{-1}$, and a phospholipid $\mathrm{P}=\mathrm{O}_{2}$ stretch $\sim 1250 \mathrm{~cm}-1$. The two- and three layer exhibited overlap in their spectra due to the layering process of the surface modifications.

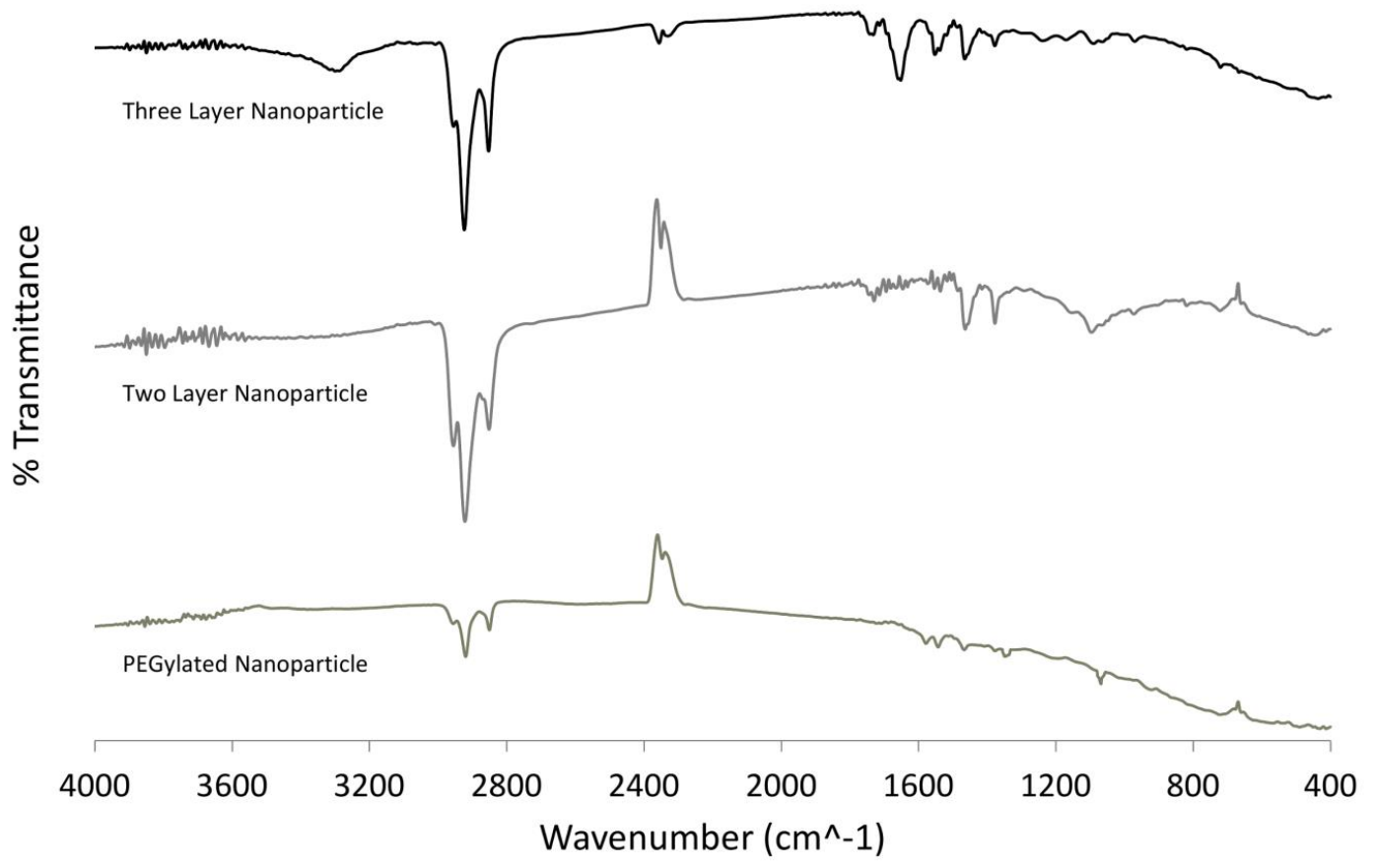

Figure 6. Determination of Surface Functionalizing using FTIR. From bottom to top: PEGylation, two layer containing dodecathiol and PC, and three layer containing dodecathiol, PC and HDL. PEGylated nanoparticles exhibited C-O-C stretch of the PEG ether $\sim 1050 \mathrm{~cm}^{-1}$ and vibration $\sim 1350 \mathrm{~cm}^{-1}$, along with $\mathrm{CH}_{2}$ and $-\mathrm{CH}_{3}$ bending vibrations around $\sim 1450 \mathrm{~cm}^{-1}$. Two Layer nanoparticles with an outer layer of PC 
exhibited $\mathrm{PO}_{4}{ }^{3-}$ group vibrations between $\sim 820-1000 \mathrm{~cm}^{-1}$, C-O-C stretch $\sim 1100 \mathrm{~cm}^{-1}$, with the largest band corresponding to the asymmetric and symmetric $-\mathrm{CH}_{2}\left(2880 \mathrm{~cm}^{-1}\right)$ and $-\mathrm{CH}_{3}\left(2950 \mathrm{~cm}^{-1}\right)$ stretch and vibration, respectively. For HDL coated nanoparticles, the asymmetric and symmetric $-\mathrm{CH}_{2}\left(2880 \mathrm{~cm}^{-1}\right)$ and $-\mathrm{CH}_{3}\left(2950 \mathrm{~cm}^{-1}\right)$ stretch and vibration are still shown along with $\mathrm{C}=\mathrm{O}$ from the lipid ester $\sim 1750 \mathrm{~cm}^{-1}$, amide bonds between 1600-1700 $\mathrm{cm}^{-1}$, and a phospholipid $\mathrm{P}=\mathrm{O}_{2}$ stretch $\sim 1250 \mathrm{~cm}^{-1}$

SEM was used to determine particle size and morphology (Figure 7). Nanoparticle shape has been demonstrated to influence particle uptake into tissues [287]. Gold nanoshells, and for the most part citrategold nanoparticles, were spherical, which is desirable to produce optimal optical properties for potential NIR treatments. PEGylated gold nanoshells had spherical morphology and were $\sim 150-170 \mathrm{~nm}$ in diameter. The influence of hydrodynamic size plays a large role in how nanoparticles will act in vivo. DLS values were determined for each type of particle with varying sizes similar to those depicted by SEM (Table 1). To ensure decreased aggregation before DLS analysis, particles were sonicated and mixed thoroughly.

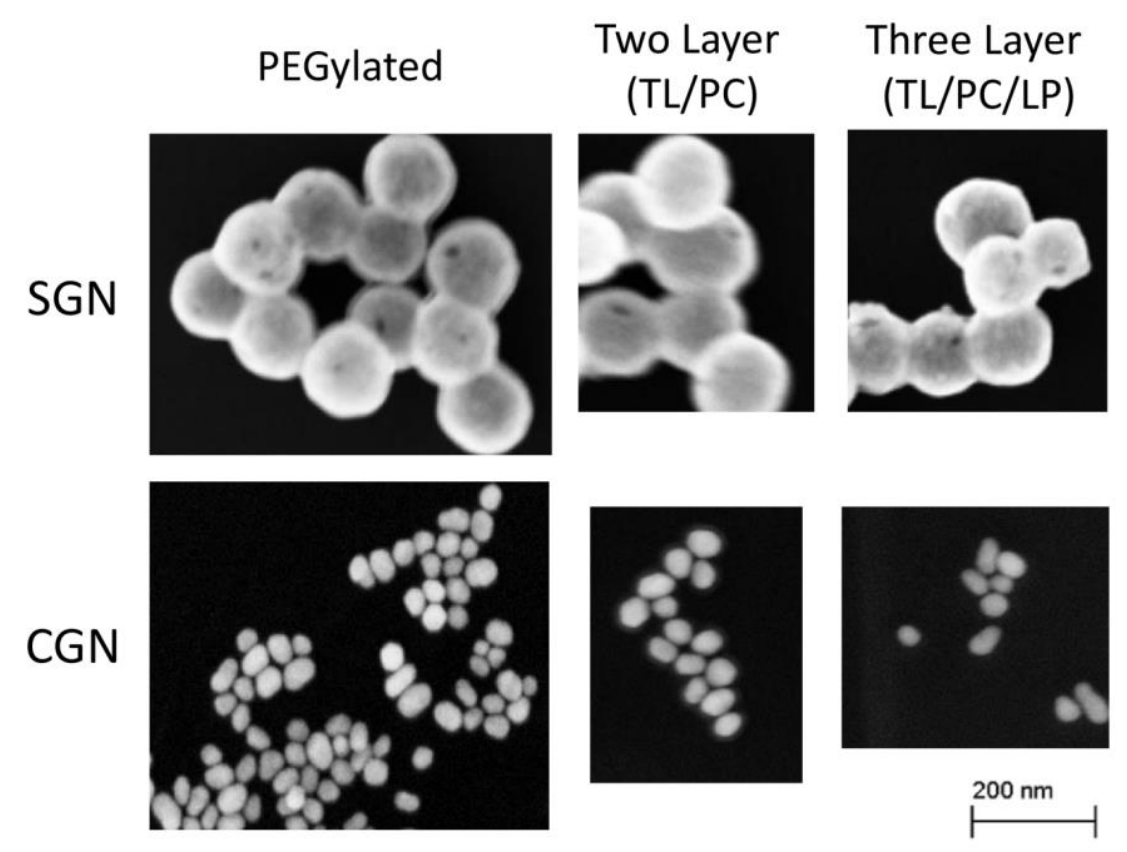

Figure 7. Scanning Electron Images of Functionalized Gold Nanoparticles. Silica gold nanoshells exhibit a particle size $\sim 150-170 \mathrm{~nm}$ in diameter. Citrate gold nanoparticles with an average diameter of $\sim 50 \mathrm{~nm}$ were 
much smaller than nanoshells, and appeared to form polydispersed clusters, with the two- and three-layer citrate gold particles being approximately the same size.

Citrate gold nanoparticles were much smaller than nanoshells (Figure 7), measuring $\sim 50 \mathrm{~nm}$ in diameter. Aggregation was more prevalent with citrate gold nanoparticles, as evidenced by the PEGylated particles (Figure 7). This aggregation is due to the decreased zeta potential of citrate gold nanoparticles when compared to the gold nanoshells. Addition of hexadecanethiol and PC is estimated to add $\sim 5-7 \mathrm{~nm}$ to the diameter of the gold nanoparticles due to the size of PC $(\sim 2.5-3 \mathrm{~nm}$ and - thiol $\sim 2.5 \mathrm{~nm})[322,323]$, while HDL is estimated to add $\sim 10 \mathrm{~nm}$ due to the size of this molecule [324].

\section{Nanoparticle Penetration in 3D Cell Cultures}

In order to determine particle penetration and distribution into $3 \mathrm{D}$ cell cultures, region of interest (ROI) measurements were taken at the periphery and center of the spheroid sections using silver enhancement staining (Figure 8). This staining is commonly used for nanoparticle detection. We also evaluated the samples using hyperspectral imaging with dark field microscopy. Results (ROI intensity) were measured between unstained and stained samples. Differences between the samples were extracted in order to obtain particle accumulation in the 3D cell cultures. 

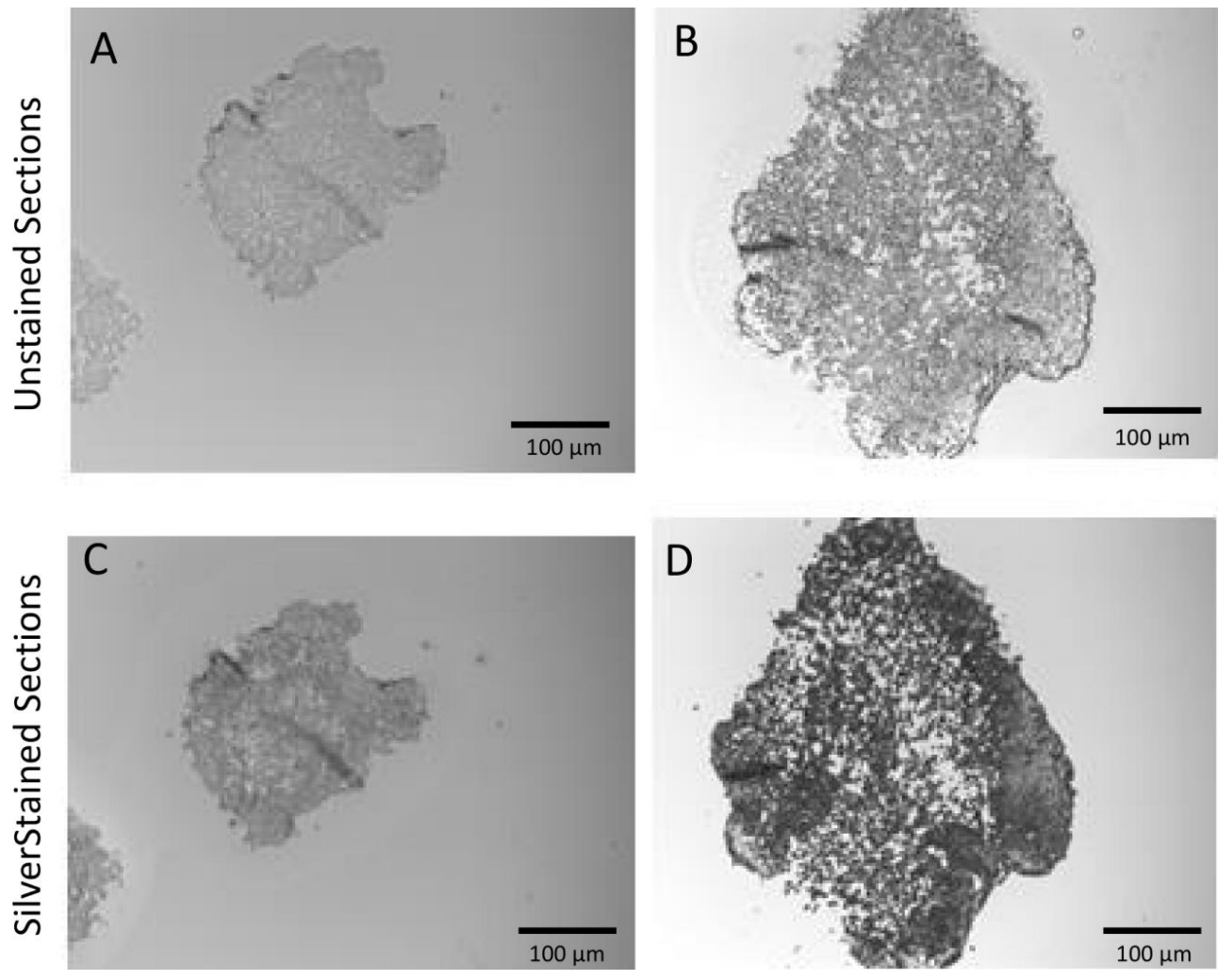

Figure 8. Nanoparticle Detection in 3D Cell Cultures through Silver Staining. ROI intensities were measured for both unstained and stained tissue sections; darker regions denote an increase in ROI intensity. Top: 20X image of A549 3D cell culture (A) before and (B) after silver staining. Bottom: 20X image of HEPG2 3D cell culture (C) before and (D) after silver staining.

For all three cell lines, the two- and three layer silica gold nanoshells staining exhibited higher ROI intensity values than the PEGylated counterpart (Figure 9). For the HEPG2 cells, the two layer nanoshells presented ROI intensity values that were statistically the same between periphery and interior, indicating uniform distribution into the tissue. This particle type had an ROI intensity nearly $2 \mathrm{x}$ that of the three layer and 3x that of the PEGylated particles (Figure 9A). The two layer citrate gold particles had a peripheral ROI intensity that was almost $2 x$ that of the interior, indicating a steep diffusion gradient into the 3D cell culture. In contrast, the three layer citrate gold particles exhibited similar intensities between periphery and interior at lower values than the silica gold nanoshells, suggesting uniform yet poorer penetration. The PEGylated particles had higher ROI intensity at the periphery than the interior while showing the lowest particle uptake. It should be noted that ROI intensity differences for the HEPG2 cell 
line were higher than both the A549 and S2VP10 cell lines, suggesting an increased amount of particles present in the HEPG2 tumors regardless of particle type.

\section{Nanoparticle Accumulation at Periphery and Center Regions of 3D Cell Cultures}
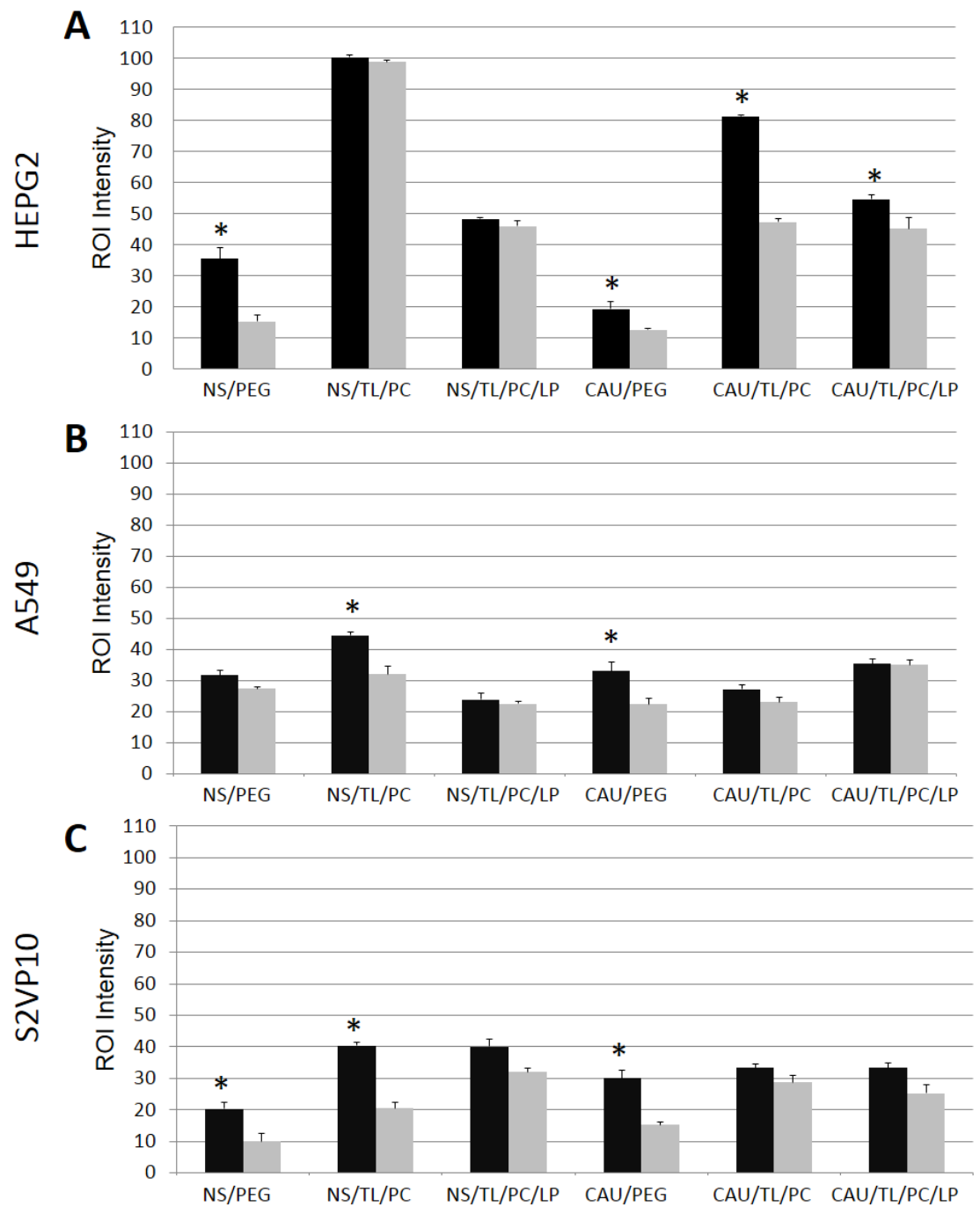

Key: 3D Cell Culture: Periphery-Black Bar (Left) and Center-Grey Bar (Right)

Figure 9. Accumulation of Gold Nanoparticles in 3D Cell Cultures by Silver Enhancement. ROI intensity measurements were taken at the periphery (black bars) and in center regions (grey bars) of HEPG2 (liver), A549 (non-small cell lung cancer), and S2VP10 (pancreatic) 3D cell cultures. Exposure to the various types of silica gold nanoshells and citrate gold nanoparticles generally exhibited equal or increased amounts of particles on the spheroid periphery as compared to the interior. All error bars represent standard deviation 
from at least $n=3$ measurements; asterisk indicates statistically significant difference $(\mathrm{p}<0.05)$ determined by Student's t-test with $\alpha=0.05$.

The ROI intensities for the A549 cell line indicated an increased accumulation of two layer compared to PEGylated silica gold nanoshells (Figure 9B). The two layer particle had higher penetration but with more of a differential between periphery and tumor interior compared to the three layer case. The three layer citrate gold particle exhibited higher ROI and more uniform values compared to the other citrate gold cases.

The S2VP10 cell line presented 2x the ROI intensity values at the periphery for both two- and three-layer silica gold nanoshells compared to the PEGylated case (Figure 9C), although the three-layer had $\sim 50 \%$ higher values than the two-layer in the interior. Both two- and three-layer silica nanoshell and citrate gold particles had significantly higher ROI in the interior than the PEGylated particles, indicating increased particle penetration. The two- and three layer citrate gold particles presented similar ROI intensity values between periphery and interior compared to the PEGylated case, suggesting a more uniform particle distribution.

Modified particles within the sectioned tumor spheroids were observable in the images captured via dark-field microscopy. The particles reflected the light, displaying sharp contrast with the surrounding tissue, and could be numerically calculated. A representative image is displayed in Figure 10, and arrows pointing to the small red-orange dots indicate the position of sample particles within the tissue. To ensure that the particles could be distinguished from the tissue, hyperspectral mapping was used to filter out the tissue while leaving nanoparticles [325]. Spectral libraries, as shown in Figure 11, were used to locate nanoparticles within the 3D cell culture. Individual nanoparticles were identified and accumulation within various spheroid regions was calculated. 

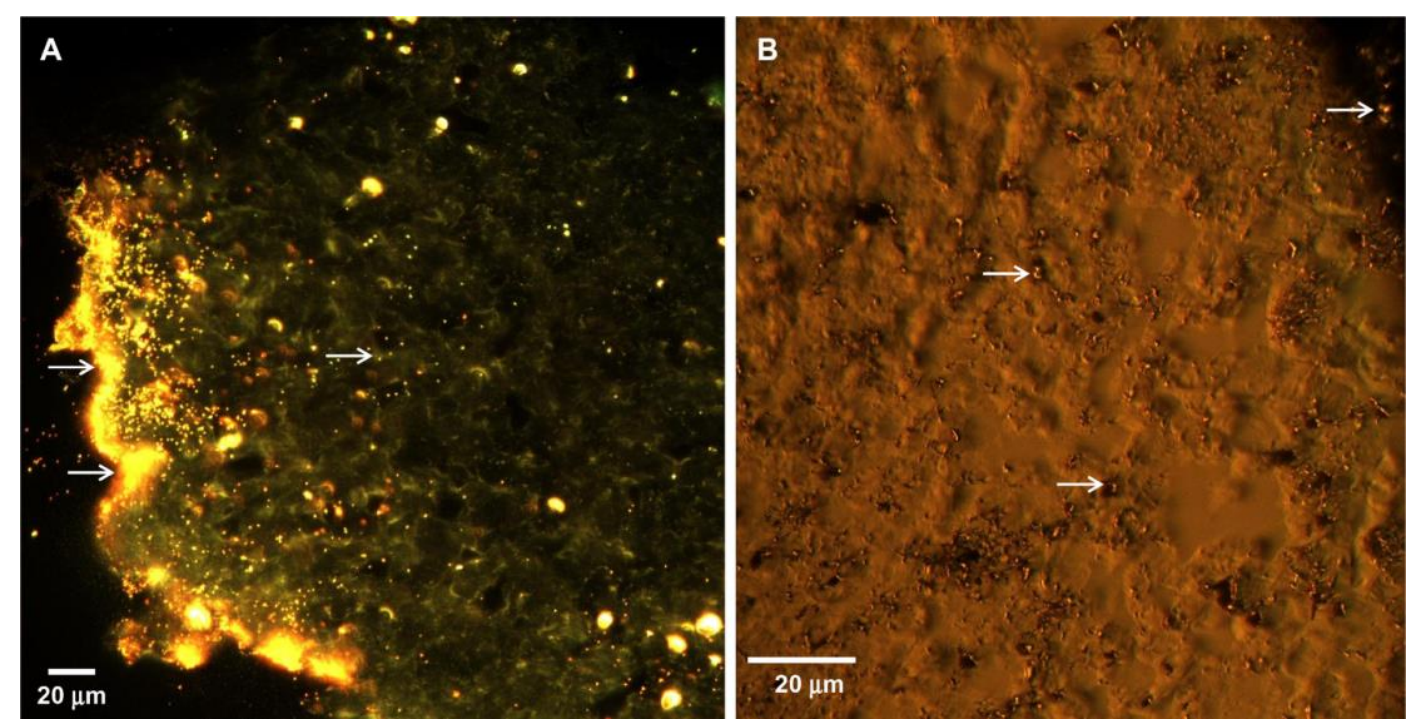

Figure 10. Detection of Nanoparticles in 3D Cell Cultures using Dark Field Microscopy. Nanoparticle uptake was determined using the CytoViva setup with a dark field microscope to visualize the nanoparticles, and with hyperspectral imaging to confirm particle identity. (A) 60x image showing increased particle concentration around periphery of HEPG2 3D cell culture with decreasing particle numbers towards the interior. (B) 100x image of a549 3D cell culture showing the particles (arrows) distributed throughout the tissue.
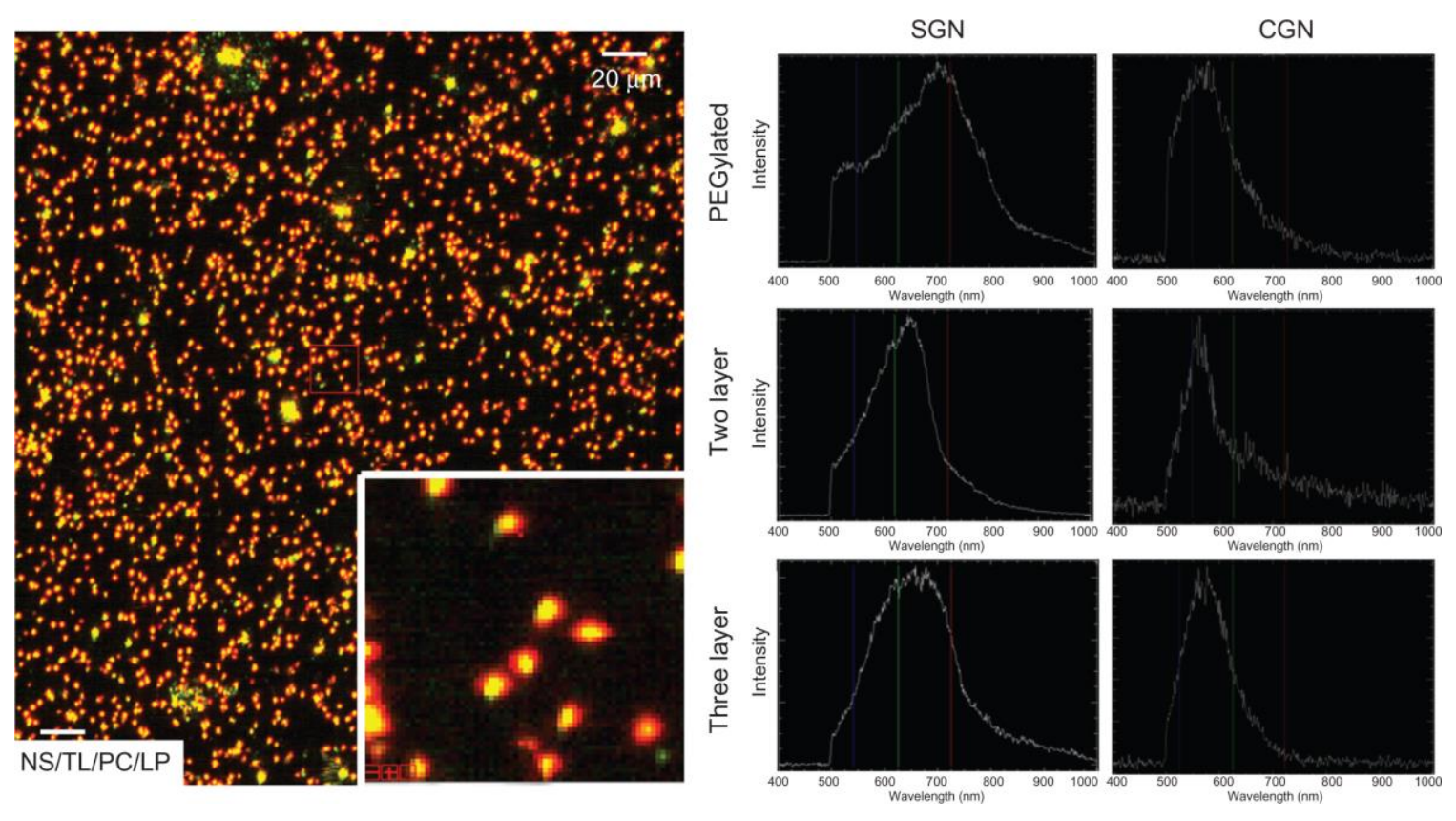
Figure 11. Hyperspectral Imaging of Nanoparticles using CytoViva Imaging System. Nanoparticles in solution were placed on slide for analysis using hyperspectral imaging. Individual particles could be identified using this technique. For further analysis of the particles, individual spectra are presented for the PEGylated, and two and three layer nanoparticles. Spectra differences are associated with varying surface modifications.

Particle uptake and penetration from the periphery of the tumor spheroids into interior regions was analyzed via counts obtained from the hyperspectral dark-field images (Figure 12). In agreement with the silver staining measurements, the two layer silica gold nanoshells in the HEPG2 tumors and the three layer citrate gold nanoparticles in the A549 tumors had the best performance in terms of higher uptake and uniform distribution, while both of these particle types behaved similarly in the S2VP10 tissue with lower uptake and steeper diffusion gradients. The three-layer particles showed slightly higher uptake into S2VP10 tissue, which could be due to the morphology of these tumors resembling a cluster of grapes, as well as other cancer-specific interactions with the HDL. Generally, the PEGylated particles exhibited lower uptake and poorer penetration. 

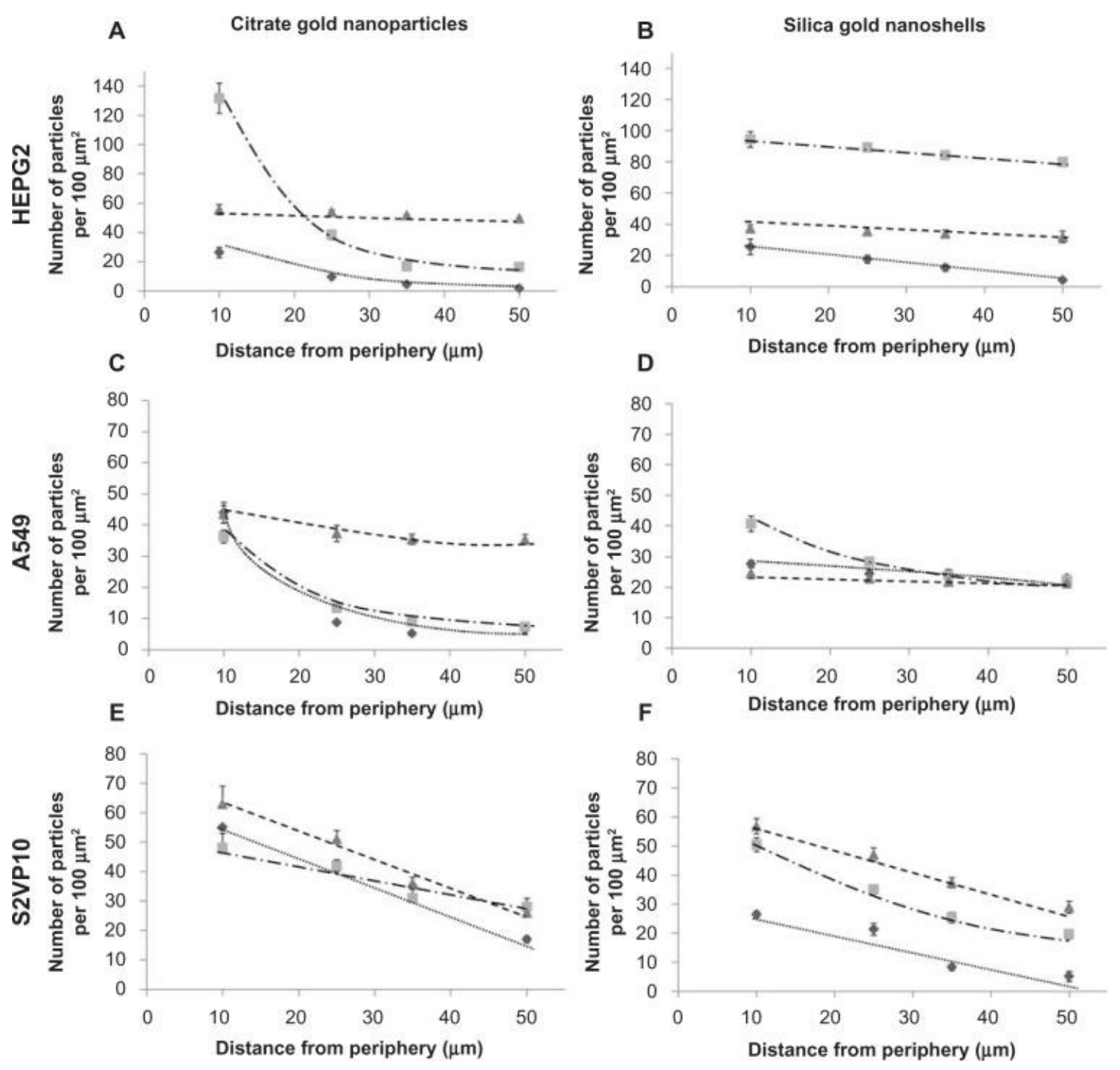

Figure 12. Nanoparticle Penetration and Distribution in 3D Cell Cultures Measured by Dark Field

Microscopy. Diamonds and dotted line: PEGylated; Squares and dashed/dotted line: two layer; Triangles and dashed line: three layer. Particle uptake was quantified for citrate gold nanoparticles (left) and silica gold nanoshells (right) for HEPG2 (liver), A549 (non-small cell lung cancer), and S2VP10 (pancreatic) 3D cell cultures as a function of distance from the spheroid periphery. The two layer silica gold nanoshells in the HEPG2 tumors and the three layer citrate gold nanoparticles in the A549 tumors had the best performance in terms of higher uptake and uniform distribution, while both of these particle types behaved similarly in the S2VP10 tissue with lower uptake and steeper diffusion gradients. Generally, the PEGylated particles exhibited lower uptake and poorer penetration. All error bars represent standard deviation from at least $\mathrm{n}=3$ measurements. 
Comparing the gold nanoshell results across cancer types, the two-layer exhibited an average 50\% higher uptake compared to the three-layer nanoshells. Citrate-gold nanoparticles had approximately $30 \%$ more penetration at the tumor periphery compared to the gold nanoshells, but this was mostly due to the larger size of the nanoshells, which are $3 x$ the size of the citrate gold particles. 


\section{Discussion}

We developed "two-" and "three-layer" nanoshells to compare the penetration and diffusion capabilities of these particles against PEGylated AuroShells ${ }^{\mathrm{TM}}$ in 3D cell cultures. To this end we used PC, which has previously been employed as a passivating agent for gold nanorods significantly reducing their cytotoxicity [326]. In addition, PC is the most abundant phospholipid within the body and can be found in all cell membranes, and is thus expected to be less immunogenic.

In order to form a layer of PC around the gold nanoshells, a hydrophobic layer must also be created. Thiol groups exhibit strong binding to gold, and can be used to displace other stabilizing ligands and adsorbed molecules to stabilize the particles. An alkanethiol possesses optimal qualities for this purpose; the hydrophobic carbon chains will interact with the fatty acids of the PC to form a properly oriented layer. Here, we theorized that such a two-layer approach would yield better biocompatibility and thus improved penetration into under-vascularized tumor regions when compared to PEGylated particles. With the two-layer method, a hydrophobic region is created between the PC and alkanethiol, which could potentially hold hydrophobic drugs to produce a more potent therapeutic agent. Further, NIR-absorbing gold nanoshells have proven effective at reducing cell viability significantly in solid tumors due to hyperthermia when treated with laser radiation [316, 327].

We find that modifying gold nanoshells with an alkanethiol and PC creates a viable two-layer nanoparticle platform that consistently outperforms the current standard, PEGylated nanoparticles, when measuring particle accumulation in 3D cell cultures. As such, two-layer nanoshells should be further considered for potential photothermal and targeted drug delivery therapies. The addition of the PC layer makes the nanoparticles similar to liposomes, which have been previously used for enhanced delivery of gold nanoparticles [328]. We hypothesize that the phospholipid similarities increased cellular uptake of these nanoparticles as compared to PEGylated nanoparticles. We also developed a three-layer system; experimental evidence suggests that HDL uptake in hepatocytes is upregulated in liver cancers [329]. Adding a layer of HDL around the two-layer particles could potentially allow the particles to penetrate deeper into liver cancers as the surface modifications make uptake by the hepatocytes more likely rather than just relying on the EPR effect for passive targeting. The enhanced uptake of HDL in pancreatic and liver cancer suggests increased cellular uptake of HDL-coated nanoparticles [330, 331]. Surface charge has 
a large effect on how nanoparticles will react in vivo. PEGylated nanoparticles were expected to exhibit slightly negative values similar to those found in literature. Two layer nanoparticles with PC were expected to exhibit highly negative zeta potentials from -15 to $-40 \mathrm{mV}$ due to the negative choline head group, while the neutral surface charge of HDL would yield particles with neutral surface charges [332]. These expectations were confirmed as shown in Figure 5.

FTIR confirmed the identity of the nanoparticles by revealing the presence of various bands. For HDL, bands associated with lipid esters (1700-1800 $\left.\mathrm{cm}^{-1}\right)$ and two distinct amide stretches ( 1500-1700 $\mathrm{cm}^{-1}$ ) were expected along with characteristics from the FTIR of PC (due the layering process of nanoparticles) [333]. PC coated nanoparticles were expected to have a large peak associated with $-\mathrm{CH}_{2}$ and $-\mathrm{CH}_{3}$ groups $\left(\sim 3000 \mathrm{~cm}^{-1}\right)$ along with phosphate group vibrations $\sim 900 \mathrm{~cm}^{-1}$ [334]. PEGylated nanoparticles were expected to exhibit decreased $-\mathrm{CH}_{2},-\mathrm{CH}_{3}$, and $-\mathrm{CH}$ out of plane bending intensities. These particles were also expected to exhibit a PEG ether stretch C-O-C between $1050-1100 \mathrm{~cm}^{-1}$ with the corresponding vibration band between $1342-1353 \mathrm{~cm}^{-1}$ [335].The bands of the two- and three layer nanoparticles were stronger than the PEGylated nanoparticles.

The penetration and diffusion capabilities of two-layer and three-layer citrate-gold nanoparticles into 3D cell cultures was also analyzed, although the application of these particles in cancer therapy is limited compared to silica-core gold nanoshells. Citrate-gold nanoparticles are much smaller than nanoshells and do not possess tunable optical properties; they also lack absorption in the NIR range and thus cannot be used for photothermal ablation therapy. Evaluation of the diffusivity of nanoparticles in 3D cell cultures was also dependent upon the morphology and stability of the cell cultures. Since HEPG2 3D cell cultures formed less tightly bound clusters, nanoparticles could enter these cultures better than the A549 and S2VP10 cell cultures. This could explain the accumulation of more nanoparticles in HEPG2 spheroids in comparison to the other cell lines.

This study focused on evaluating the uptake and distribution of nanoparticles within avascular/hypoxic regions of solid tumors at varying depths of penetration using 3D cell cultures as an in vitro model of avascular tumor tissue. By quantifying the amount of particles within specific regions of tissue, the diffusion of particles can be analyzed and this information combined with mathematical modeling to better understand effectiveness of these particles for cancer treatment. The results could then 
be utilized to calibrate computational simulations to elucidate the complex dynamics of particles and drug molecules within solid tumors [292, 296, 336, 337]. The work here represents an initial step to assess the performance of two- and three-layered nanoparticles in penetrating avascular tissue. We note that 3D cell culture is a rudimentary model of avascular tumor tissue; also, the reticuloendothelial system (RES) and other systemic factors may significantly affect nanoparticle performance in vivo.

This study can be continued by examining the efficacy of the two-layer nanoshell system in photothermal applications and determining its benefits compared to PEGylated nanoshells. In addition, work is currently in progress to investigate the potential for embedding drugs into the layers of the twolayer nanoshell for chemotherapeutic delivery. A more interesting approach would be to combine the photothermal treatments with drug delivery. A system could potentially be designed that would release its drug payload once the particle has been excited through NIR energy, disrupting the layers on the nanoshell. These steps would provide a logical progression towards developing more efficacious systems for targeted cancer therapy. 


\title{
CHAPTER III
}

\section{DETECTION OF PHOSPHATIDYLCHOLINE-COATED NANOPARTICLES IN ORTHOTOPIC PANCREATIC CANCER USING HYPERSPECTRAL IMAGING}

\author{
$\underline{\text { Background }}$
}

Systemic Delivery of Nanoparticles to Tumors

Systemic delivery of nanoparticles to solid tumors can be considered in three distinct stages: systemic travel while avoiding sequestering by the reticuloendothelial system (RES, mainly the liver and the spleen), extravasation from intra-tumoral capillaries, and diffusion to reach and penetrate malignant cells $[338,339]$. The compromise of any of the associated processes can lead to suboptimal nanoparticle uptake within tumor tissue, resulting in diminished therapeutic or diagnostic efficacy. To facilitate systemic travel, surface modifications have been applied to nanoparticles to enhance passive and active targeting while minimizing RES sequestering, e.g., coating with the hydrophilic polymer poly(ethylene)-glycol (PEG) results in prolonged circulation time and diminished RES uptake [340, 341]. Coating with targeting ligands specific to over-expressed receptors on cancer cells (e.g., high-density lipoprotein receptor (HDLR)) can further enhance nanoparticle uptake and accumulation at the tumor site [341].

However, the typically irregular tumor vasculature resulting from uncoordinated pro- and antiangiogenic stimuli further hinders nanoparticle passive transport to cellular targets as well as therapeutic efficacy even if systemic travel and targeted accumulation were successful [342]. Hypo-vascularized regions of tissue lead to cells invoking survival mechanisms to overcome oxygen and nutrient deprivation including minimization of metabolism (quiescence) resulting in cell cycle-dependent chemotherapeutic resistance [343]. In addition to intrinsic and microenvironment-associated resistance mechanisms, cells distant from vasculature in solid tumors receive suboptimal levels of diffusible substances as transport may be limited by increased interstitial fluid pressure (IFP), a chaotic and dense ECM, and an acidic microenvironment [338, 342, 344-346]. 
To overcome these transport barriers and successfully deliver nanoparticles more homogeneously into tumor tissue, nanoparticle size and surface modifications have traditionally been modulated [292]. Studies have described positive correlations between $50 \mathrm{~nm}$ nanoparticles and enhanced tumor accumulation and diffusivity within tumor tissue [347], while larger $100 \mathrm{~nm}$ nanoparticles are readily filtered by the liver and their transport is hindered once at the tumor due to a dense ECM $[299,348]$. Very small $(5 \mathrm{~nm})$ gold nanoparticles conjugated with anti-EGFR antibody were shown to successfully target and treat pancreatic cancer when injected intraperitoneally into mice [349]. Using 3D cell cultures, sizespecific localization of nanoparticles was demonstrated by showing that small nanoparticles ( 2 and $6 \mathrm{~nm}$ ) exhibited superior penetration in comparison to slightly larger $(15 \mathrm{~nm})$ nanoparticles [24]. It was also shown that nanoparticles $\sim 20 \mathrm{~nm}$ displayed superior penetration in comparison to even larger nanoparticles [299].

Previously, we have shown that smaller citrate gold nanoparticles $(45-60 \mathrm{~nm})$ display enhanced tissue diffusivity in comparison to larger silica-gold nanoshells (160-175 nm) [225]. We also evaluated the effects of surface modifications on passive transport by functionalizing nanoparticles with PC and HDL. Results showed that both PC-coated and HDL-coated nanoparticles displayed enhanced tissue penetration compared to PEGylated nanoparticles in 3D cell cultures of human pancreatic, lung, and hepatocellular cancers [225]. As PEGylation is the most common surface modification, the results demonstrated that such alternative surface modifications might enhance passive transport through solid tumor tissue.

\section{Detecting Nanoparticles in Tissue}

Nanoparticle localization is usually assessed using SEM and transmission electron microscopy (TEM), yet these methods alone cannot conclusively detect nanoparticles. In combination with other tools (e.g., energy dispersive X-ray (EDX) microanalysis), elemental analysis can provide further confirmation of nanoparticle identity [350]. Here, we employ hyperspectral imaging to create libraries of known nanoparticles and thus enable their detection within tissue samples or individual cells. Recently, the deposition patterns of cisplatin aerosol therapy in surgically resected stage II lymph nodes from lung cancer patients was analyzed using hyperspectral imaging [351]. Further, recombinant human epidermal growth factor was encapsulated into liposomes, from which the morphology and particle distribution was analyzed using hyperspectral imaging [352]. This imaging has also been utilized in patients for rapid, on-sight 
histological classification of lung cancer [353], demonstrating the flexibility of this technique in biomedical applications. While nanoparticles can be identified using dark field microscopy alone, the combination of hyperspectral imaging with dark field microscopy allows for automatic detection of nanoparticles within the tissue, leading to less humanized error .

\section{Purpose of Study}

We examine the uptake and diffusivity of PC-coated citrate gold nanoparticles and silica-gold nanoshells in tumors of the pancreas. Pancreatic cancer is the fourth most common cause of cancer-related death in the United States with dismal 5-year relative survival rates of $<6 \%$ [56]. Due to the late onset of clinical symptoms and a paucity of known biomarker candidates, pancreatic cancer prevention and diagnosis remain difficult, with over $70 \%$ of pancreatic cancer cases diagnosed at stage III or IV, when surgical intervention is generally no longer an option as the disease has spread through metastasis [354]. Current treatment options fail to effectively cure pancreatic tumors and have only provided minimal increases in survival rates, demonstrating the vital need for novel treatment options. Here, we synthesize and characterize PC-coated gold nanoparticles using methods outlined previously [225], and tail-vein inject them into mice bearing orthotopic pancreatic tumors.

An orthotopic model was chosen for these experiments because it provides a more realistic comparison to the human pancreatic tumors in comparison to subcutaneous models. After allowing for 48 hours of circulation, the animals were euthanized and the organs harvested for histological analysis. Ex vivo identification and localization of nanoparticles in the liver, spleen, and pancreatic tumor tissues were determined using hyperspectral imaging of histology sections. Results were compared to previous work in which uptake of these nanoparticles was assessed in 3D cell culture [225].

\section{$\underline{\text { Methods }}$}

\section{Synthesis of Gold Nanoparticles}

As previously described [225], nanoparticles were synthesized using the method in which gold chloroauric acid is reduced by trisodium citrate [306]. In this process, $2.2-2.4 \mathrm{~mL} 1 \%$ weight/volume 
(wt/v) sodium citrate (Fisher Scientific, Waltham, MA, USA) and $200 \mathrm{~mL} 0.01 \% \mathrm{wt} / \mathrm{v} \mathrm{HAuCl} 4\left(\mathrm{Alfa}_{4}\right.$ Aesar, Ward Hill, MA, USA) are mixed and heated to boiling, which promotes the reaction of sodium citrate to citric acid. Temperature and final concentration of the gold salt allows particles of varying sizes. Once the reaction is completed, the solution is concentrated using a rotovapor (Buchi Rotovapor System, BÜCHI Labortechnik AG, Flawil, Switzerland) to $\sim 20 \mathrm{~mL}$ before the addition of layering to the particles.

The silica gold nanoparticles have an inner core composed of silica with an outer coating of gold. Synthesis consists of four stages: production of a colloid of small gold particles $(2-4 \mathrm{~nm})$ through reduction and aging of gold colloid produced by the recipe of Vogel et al. [355], fabrication of monodispersed silica cores from the Stöber method [232, 308], attachment the seeds to the silica surface, and finally gold shell growth via reduction of additional gold. The gold colloid solution is created utilizing the THPC (Tetrakis(hydroxymethyl)phosphonium chloride) method [316]. Growth of the silica cores requires the combination of $7.5 \mathrm{~mL}$ tetraethyl-orthosilicate (TEOS, Sigma Aldrich, St Louis, MO, USA), $225 \mathrm{~mL}$ absolute ethanol (Decon Labs, King of Prussia, PA, USA), and 12.5-13.5 mL ammonia (Sigma Aldrich). Ammonia is adjusted to achieve silica core sizes $110 \pm 5 \mathrm{~nm}$. After removal of the paraffin cover and evaporation of the ammonia, the cores are coated with 3\%-4\% aminopropyltriethoxysilane (APTES; Sigma Aldrich). This allows for slightly positive cores for deposition of small colloidal gold particles, thus forming what is hence called a seed particle.

The seeds are then washed and a $10 \%$ gold solution is added to complete the shell. After reaction time, the seeds are washed and re-dispersed in DI water. The seeds are diluted to $0.3-0.5$ optical density (OD) at $530 \mathrm{~nm}$ (Varian Cary 50 Bio UV-Visible Spectrometer, McKinley Scientific, Sparta, NJ, USA). A sweep of the seeds is performed to optimize the chemical ratio between them, $\mathrm{K}_{2} \mathrm{CO}_{3}-\mathrm{HAuCl}_{4}$, and formaldehyde (Fisher Scientific). Limiting the concentration of gold in the final reduction step controls the thickness of the gold shell.

\section{Functionalization of Nanoparticles}

The first layer applied to the citrate gold nanoparticles and silica-gold nanoshells was 1Hexadecanethiol (Sigma Aldrich) dissolved in ethanol. While stirring, $20 \mathrm{~mL}$ pure ethanol (Decon Labs) was placed in a beaker with $60 \mu \mathrm{L} 1$-Hexadecanethiol being added secondly. The nanoparticles were added to the sample slowly over the next few minutes. The sample was first agitated via shaking and sonication 
for 60 minutes, and then placed for 12 hours on an orbital rocker (Boekel Scientific, Feasterville, PA, USA) overnight. The sample was spun down, and the pellet was washed twice and resuspended in chloroform (Sigma Aldrich). The second functionalization was the addition of the PC. The stock solution was made by diluting PC in chloroform, and $100 \mu \mathrm{L}$ were added to the particles after the thiol layer and allowed to set overnight on an orbital rocker. The solutions were transferred to glass tubes and the chloroform evaporated at ambient temperature. After removal of chloroform, PC-coated citrate gold nanoparticles and silica-gold nanoshells were reconstituted in $\mathrm{ddH}_{2} \mathrm{O}$ to $2 \mathrm{OD}$.

\section{Nanoparticle Characterization}

Nanoparticle maximum absorption wavelengths were obtained using the Varian Cary 50 Bio UVVisible Spectrometer (McKinley Scientific). Nanoparticle size and zeta potential measurements were obtained using the Zeta-Sizer Nanoseries ZS90 (Malvern Instruments, Worcestshire, UK). Hydrodynamic size in solution based upon Brownian motion was measured using DLS (dynamic light scattering). Shape and size were also previously determined using SEM, with the presence of lipids on the particle cores confirmed using a FTIR spectroscopy (England et al. 2013).

\section{Cell Culture Experiments}

The highly metastatic pancreatic adenocarcinoma cell line, S2VP10 cells expressing luciferase, was obtained from Dr. Michael Hollingsworth (University of Nebraska). Cells were grown in DMEM with $10 \% \mathrm{FBS}$ and $1 \% \mathrm{~L}$-glutamine at $37^{\circ} \mathrm{C}$ in a humidified incubator.

\section{Human Pancreatic Cancer Orthotopic Xenograft Mouse Model}

Adherence to the University of Louisville Institutional Care and Use Committee (IAUCUC) approved protocol was upheld for the in vivo experiments. Severe combined immunodeficiency (SCID) female mice (Harlan, Indianapolis, IN) received orthotopic pancreatic injections of S2VP10 metastatic pancreatic adenocarcinoma cells expressing luciferase, resulting in pancreatic tumors within 7 days. The procedure for orthotopic pancreatic cell implantation was previously described (McNally et al. 2010). Briefly, mice were anesthetized with isoflurane ( $\leq 4 \%$ isoflurane for induction and $\sim 1.5 \%$ maintenance dose) at $100 \% \mathrm{O}_{2}$. The left upper abdominal quadrant of the animals was sterilized before making a 1-cm incision. The pancreas was localized using forceps, and the tail of the organ was injected with $1.5 \times 10^{5}$ cells/30 $\mu \mathrm{L}$ S2VP10 cells expressing luciferase using a 28 -gage needle. Peritoneal leakage of cell solution 
from pancreatic injection site was minimized by applying a sterile cotton tip applicator for 30 seconds. Organs were returned to normal anatomical position prior to closing the skin and peritoneum using 5-0 Nylon sutures. Animals recovered in a warm area and received liquid acetaminophen for 24 hours postsurgery. Growth of the tumor was monitored using the AMI-1000X bioluminescence imaging system (Spectral Imaging Instruments, Tucson, AZ). $2.5 \mathrm{mg}$ luciferin was given via intraparenteral injection to each mouse 10 minutes prior to imaging.

An intravenous (tail vein) injection of $200 \mu \mathrm{L} 2$ OD nanoparticle solutions was given to the mice nine days after tumor cell implantation. As there were five mice in each group, the first group received an injection of PC-coated citrate gold nanoparticles; the second group mice received an injection of PC-coated silica-gold nanoshells, and the third group was the negative control (no particle injection).

Mouse Euthanasia, Organ Resection, and Histological Processing

Nanoparticles were allowed to circulate for 48 hours before mice were euthanized using $\mathrm{CO} 2$. The pancreatic tumor, liver, and spleen were removed from each mouse for histological processing. Histology was performed by the University of Louisville Pathology Laboratory (Louisville, KY, USA). Tissues were cut into $4 \mu \mathrm{m}$ sections and placed onto slides before undergoing a series of ethanol and xylene washes. Cover slips were applied before experimental analysis.

\section{Nanoparticle Detection using Hyperspectral Imaging}

Hyperspectral imaging in combination with dark field microscopy was used to assess nanoparticle uptake and distribution within histology tissue sections. The CytoViva® Hyperspectral Imaging System (CytoViva Inc., Auburn, AL, USA) was used for this purpose. This system uses a Dage camera with a microscope with dark field capability. Hyperspectral profiles are acquired using a Pixelfly camera and visualized using ENVI 4.8 software (Exelis Visual Information Solutions, Boulder, CO, USA). To confirm the identity of nanoparticles, spectral libraries were created using z-spectral profiles and compared to the tissue samples. We found that spectral mapping was able to detect and confirm the nanoparticles within tissue samples by using multiple tissue images (> 10) containing either nanoparticles or no nanoparticles. Data analysis was performed with ImageJ. Regions of interest were randomly determined and particles within these regions were counted to determine the concentrations in liver, spleen, and pancreatic tumor 
tissues. A sufficient number of regions were evaluated to ensure at least $90 \%$ accuracy based on stereological analysis.

\section{Statistical Analysis}

Analyses used the two-tailed Student's t-test with significant values being less than 0.05 . Statistically significant differences are illustrated with an asterisk $(*)$ in the results. 


\section{$\underline{\text { Results }}$}

\section{Nanoparticle Synthesis and Characterization}

The addition of a PC layer to citrate gold nanoparticles and silica-gold nanoshells was accomplished using a layering process in which the charged head group of hexadecanethiol binds to the nanoparticle (Figure 13), resulting in hydrophobic nanoparticles with hydrocarbon chains pointed outward towards the surrounding environment. The addition of PC to the hexadecanethiol-coated nanoparticles binds tail-to-tail creating water-soluble nanoparticles containing an inner layer suitable for hydrophobic drug loading.

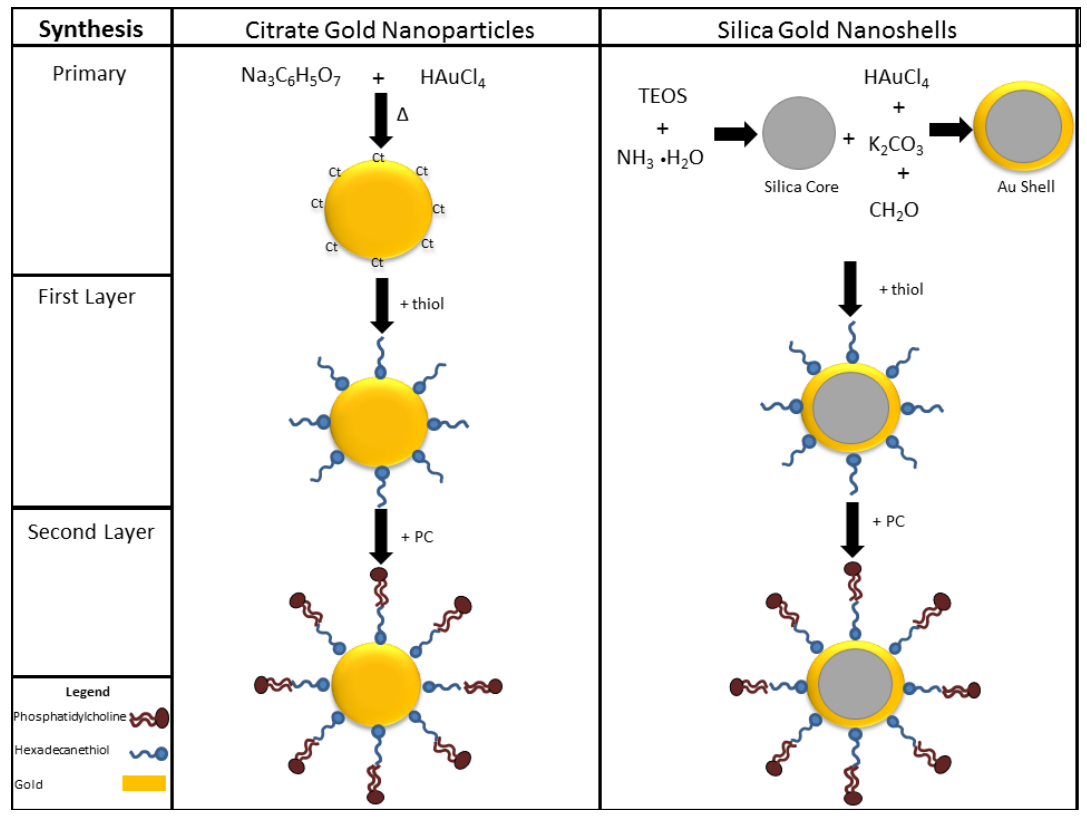

Figure 13. Synthesis of Two and Three Layer Nanoparticles. Briefly, citrate gold nanoparticles were synthesized by the reduction of chloroauric acid by sodium citrate. The citrate is removed from the surface by the addition of hexadecanethiol, which is used for the conjugation of PC. Silica-gold nanoparticles were synthesized by first creating silica cores before adding ultra-small colloidal gold nanoparticles onto the surface of the cores to create a thin layer of gold through a reduction process. The same modifications of hexadecanethiol and PC were applied to the silica-gold nanoshells. TEOS: tetraethyl-orthosilicate.

Optical measurements of functionalized citrate gold nanoparticles and silica-gold nanoshells confirmed nanoparticle identity and functionalization (Figure 14). Colloidal gold nanoparticles exhibit UV- 
Vis maximum absorbance values ranging between $510-550 \mathrm{~nm}$, in agreement with the PC-coated nanoparticles experiencing an optimal peak at $540 \mathrm{~nm}$ (Figure 14A). In comparison, previously synthesized PEGylated citrate gold nanoparticles displayed a maximum wavelength of $533 \mathrm{~nm}$ [225]. Maximum absorbance of silica-gold nanoparticles is based upon the radius of the silica core and size of the gold shell surrounding the core, with smaller shells producing larger wavelengths. Silica-gold nanoshells synthesized in this study displayed a maximum absorbance of $835 \mathrm{~nm}$ that is similar to silica gold nanoparticles with $\sim 110 \mathrm{~nm}$ diameter silica cores and $\sim 10-15 \mathrm{~nm}$ gold coating (Figure 14B). In comparison, previously synthesized PEGylated silica-gold nanoshells displayed a maximum absorbance of $820 \mathrm{~nm}$. We have previously shown that silica-gold nanoparticles commonly possess wavelengths ranging from $820-860 \mathrm{~nm}$ based upon sizing and surface modifications [225].

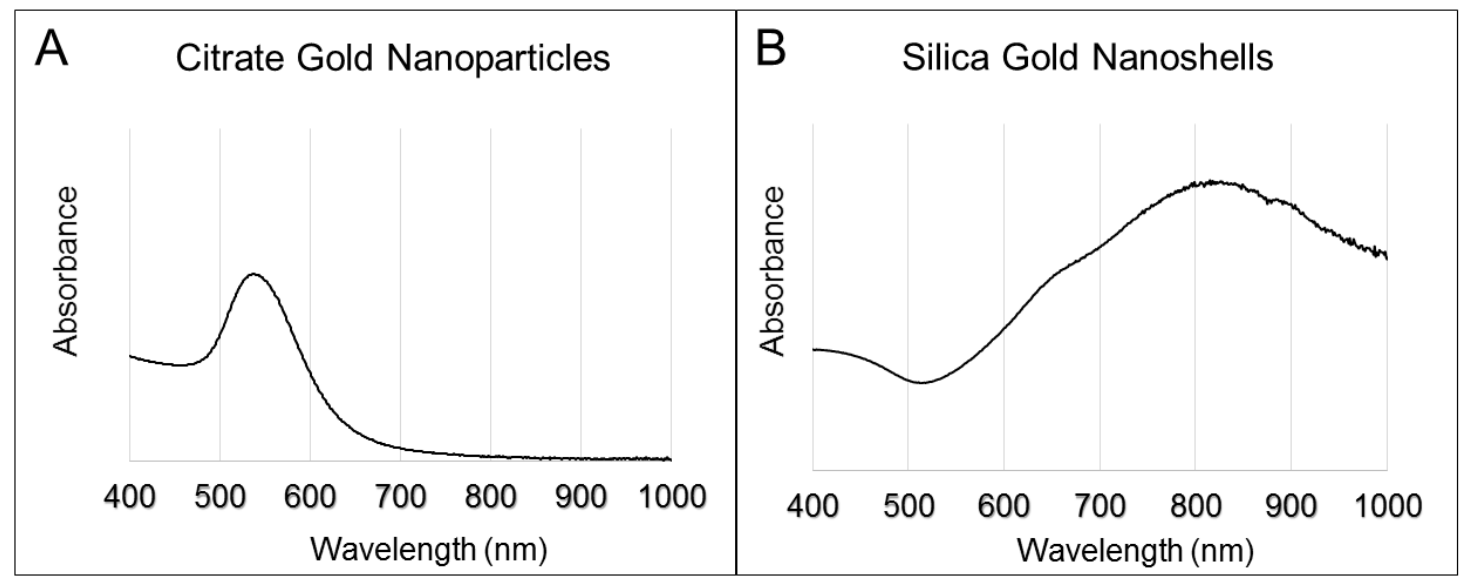

Figure 14. UV-Visible Spectroscopy Spectra of Nanoparticles. (A) Citrate gold nanoparticles displayed a maximum absorption at $540 \mathrm{~nm}$. (B) Silica-gold nanoshells displayed a maximum absorption at $830 \mathrm{~nm}$.

Further characterization elucidated information regarding hydrodynamic radii and surface charge (Figure 15). The hydrodynamic radius has a crucial impact on deposition patterns in vivo as a thin electric dipole layer of the solvent adheres to the surface of the nanoparticles as they move through a liquid medium. The size determined through DLS was greater than the sizing information obtained visually by SEM, which is a common occurrence. The PC-coated citrate gold nanoparticles had an average DLS size of $82.3 \pm 9.4 \mathrm{~nm}$ (Figure 15A), while PC-coated silica-gold nanoparticles had an average size of $144.13 \pm 11.7$ 
$\mathrm{nm}$ (Figure 15B). These DLS sizes can be contrasted with previously synthesized PEGylated citrate gold nanoparticles and silica-gold nanoshells exhibiting sizes of $82.81 \pm 13.4 \mathrm{~nm}$ and $161.82 \pm 12.2 \mathrm{~nm}$,

respectively. The size difference between citrate gold and gold nanoshells is expected to have an impact on diffusivity within in vivo tumor tissue, in accordance with previous observations using 3D cell culture [225]. The surface charge showed similar zeta potentials between the citrate gold nanoparticles and silicagold nanoshells of $-23 \mathrm{mV}$ (Figure 16A) and $-31 \mathrm{mV}$ (Figure 16B), respectively.

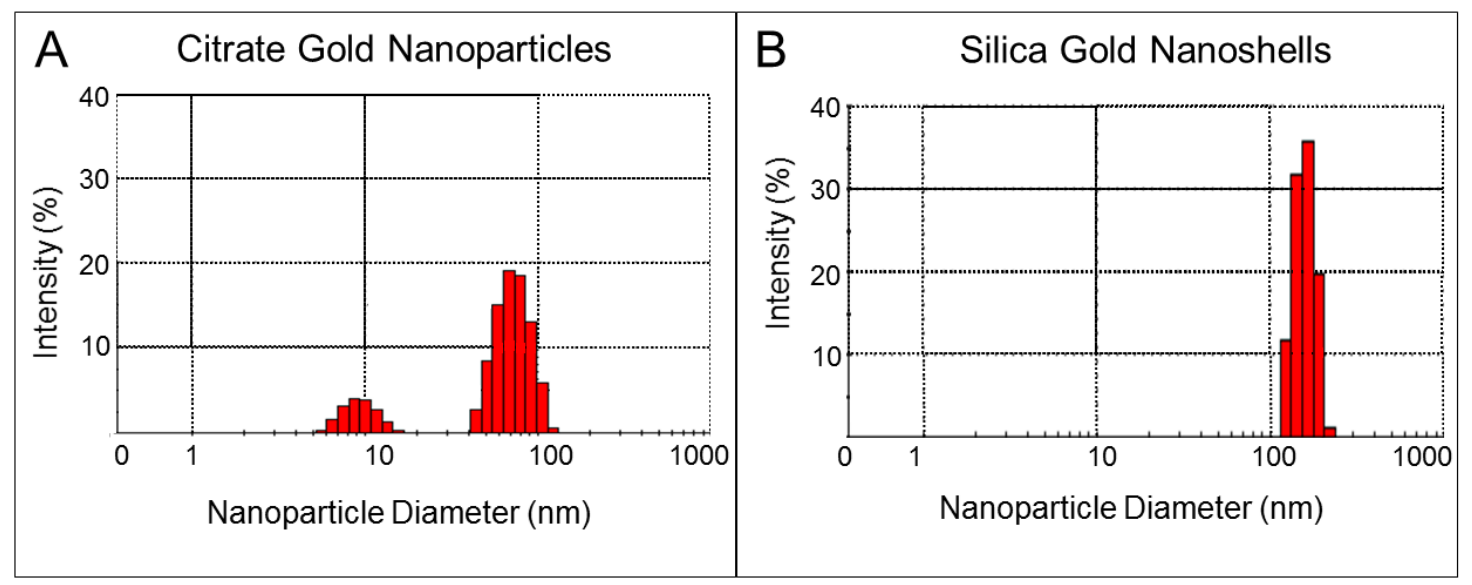

Figure 15. Hydrodynamic Radius of Nanoparticles Measured using DLS. (A) The radius of PC-coated citrate gold nanoparticles was $82.3 \pm 9.4 \mathrm{~nm}$. The hydrodynamic radius of PC-coated silica gold nanoparticles was $144.13 \pm 11.7 \mathrm{~nm}$.

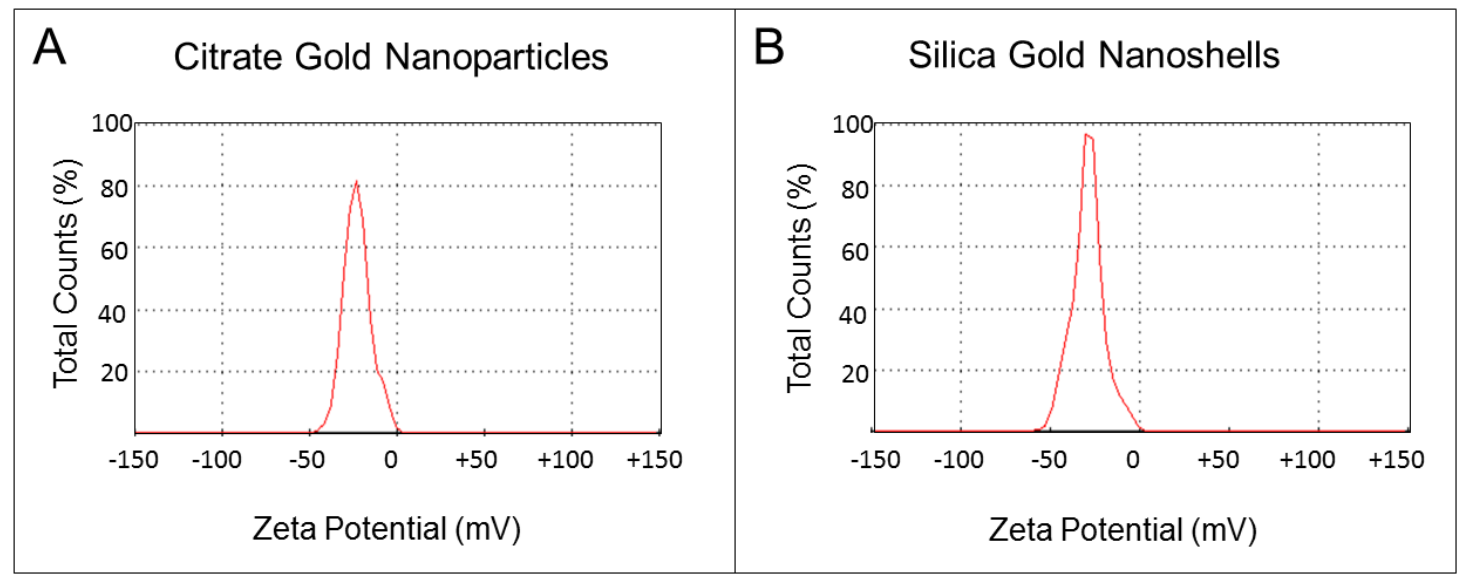

Figure 16. Surface Charge of Nanoparticles Measured using the Zetasizer. The surface charge, or zeta potential, of these nanoparticles displayed similar cationic surfaces with (A) the PC-coated citrate gold 
nanoparticles possessing a zeta potential of $-23 \mathrm{mV}$. The PC-coated silica-gold nanoshells were slightly more cationic at $-31 \mathrm{mV}$.

\section{Creation of Nanoparticle Spectral Libraries}

Before nanoparticles were detected in tissue samples from mice, spectral libraries were created of the PC-coated citrate gold nanoparticles and silica-gold nanoshells that could be later matched to the images for detection (Figure 17). The spectral libraries were synthesized from samples of both types of nanoparticles in solution. Visible color changes from green to yellow to red illustrated the shift of the maximum absorbance wavelength of the nanoparticles. For PC-coated citrate gold nanoparticles, the majority of nanoparticles in solution exhibited green color indicating the particles processed maximum absorbance values within the lower range (500-600 nm) (Figure 17A). This was confirmed through the zprofile spectra mapping showing a maximum band $\sim 550-570 \mathrm{~nm}$. The solution of PC-coated silica-gold nanoshells contained a significant concentration of red nanoparticles, indicative of absorbance at higher wavelengths (>700 nm) (Figure 17B). As nanoshells exhibit wavelengths higher than colloidal gold nanoshells, this also helped to confirm the identity of the nanoparticles.
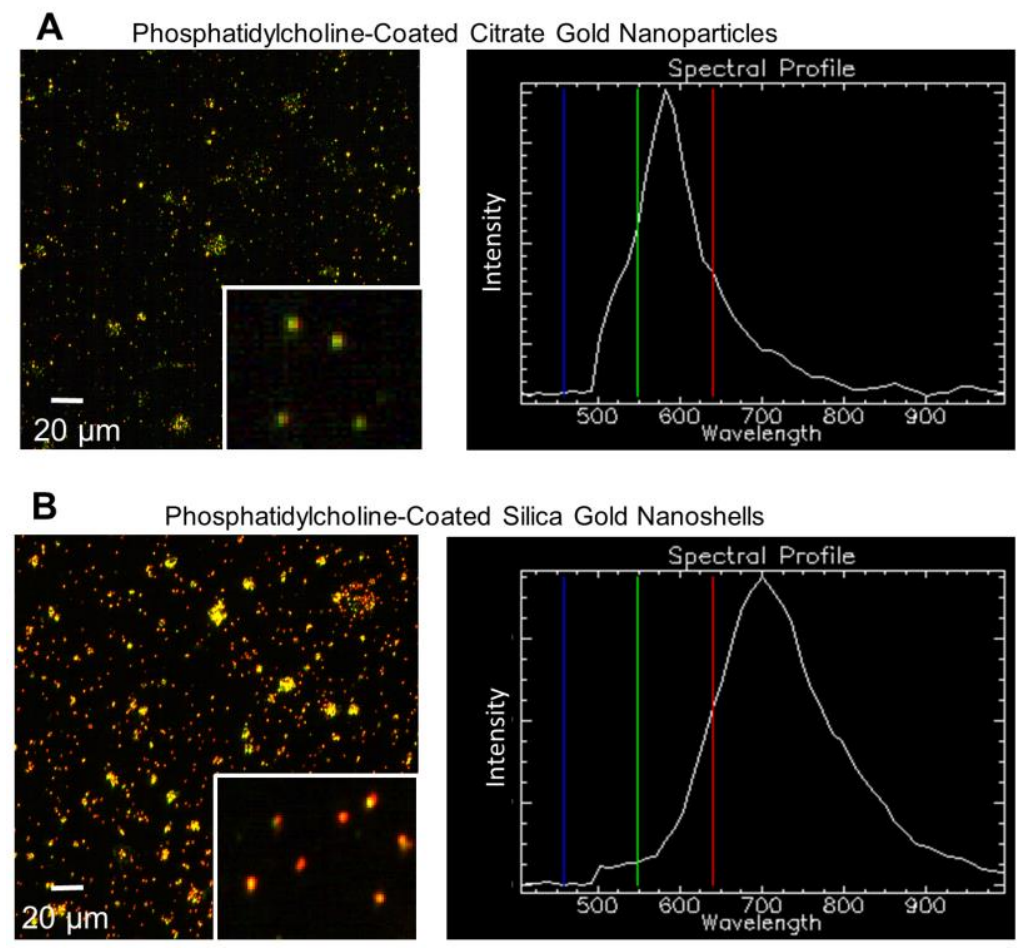
Figure 17. Hyperspectral Imaging of PC-coated Gold Nanoparticles. (A) Citrate gold nanoparticles were primarily green, with a spectral profile indicating a maximum wavelength of $570 \mathrm{~nm}$. (B) Silica-gold nanoshells displayed an abundance of red and yellow nanoparticles, with a spectral profile maximum of $700 \mathrm{~nm}$. While the actual maximum wavelength of silica-gold nanoshells in solution is between 820-860 $\mathrm{nm}$, hyperspectral imaging is known to underestimate entities containing higher wavelength maxima. Size bar corresponds to $20 \mu \mathrm{m}$.

\section{Evaluation of Nanoparticle Uptake and Distribution}

The creation of spectral libraries of PC-coated nanoparticles enables nanoparticle detection in tissue samples. After nanoparticles extravasate from vasculature, they diffuse through the tissue composed of cells and ECM. In some tissues, extravasation sources residing relatively close to each other can provide a more homogenous layout of source points. Nanoparticle counts were obtained in the vicinity of source points in histology sections to determine the uptake and distribution in sections of liver, spleen, and orthotopic pancreatic tumor (Figure 18). 

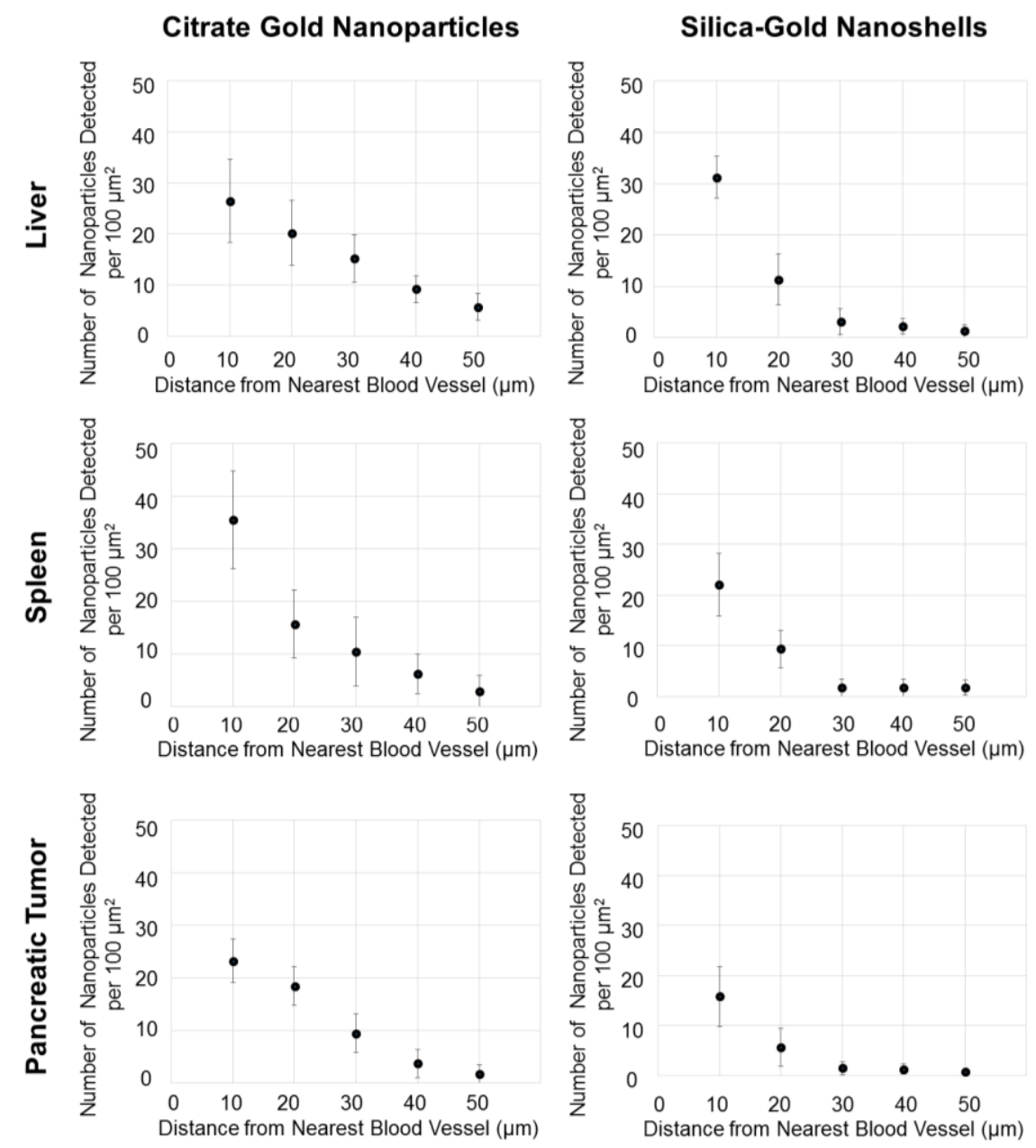

Figure 18. Nanoparticle Penetration in Liver, Spleen, and Pancreatic Tumor. Citrate gold nanoparticle and silica-gold nanoshell penetration measured in liver, spleen, and pancreatic tumor tissue samples. Distances from the nearest source were measured using hyperspectral imaging. In the liver and pancreatic tumor, citrate gold nanoparticles had a linear diffusion pattern, while the spleen had an exponential decline. Silica gold nanoshells had an exponential decline, suggesting limited diffusivity in all three tissue types. Citrate gold nanoparticles had highest extravasation close to their sources in the spleen while for silica gold nanoshells extravasation was highest in the liver. All error bars denote standard deviation $(n=3)$.

For citrate gold nanoparticles, the liver and pancreatic tumor on average exhibited a linear decline in nanoparticle concentration from the nearest source, with $26.5 \pm 8.2$ and $23.3 \pm 4.1$ particles $/ 100 \mu \mathrm{m}^{2}$, respectively, within $10 \mu \mathrm{m}$ of the source and few nanoparticles penetrating beyond $50 \mu \mathrm{m}$ (Figure 18). The 
spleen displayed on average a higher uptake of $35.5 \pm 9.3$ particles $/ 100 \mu \mathrm{m}^{2}$ within $10 \mu \mathrm{m}$ followed by an exponentially decaying penetration pattern, also limited to $\sim 50 \mu \mathrm{m}$ (Figure 18).

Silica-gold nanoshell were uptaken the most within the liver showing on average $31.1 \pm 4.1$ particles $/ 100 \mu \mathrm{m}^{2}$ within $10 \mu \mathrm{m}$ of the nearest source, with penetration hindered beyond $30 \mu \mathrm{m}$ (Figure 18). The spleen and pancreatic tumor also exhibited limited penetration beyond $30 \mu \mathrm{m}$ (Figure 18), with uptake of $22.1 \pm 6.2$ and $15.8 \pm 6.1$ particles $/ 100 \mu \mathrm{m}^{2}$, respectively, within $10 \mu \mathrm{m}$. While the penetration of citrate gold nanoparticles suggests linear diffusion, the nanoshells displayed more of a decaying exponential pattern further implying limited diffusivity. We hypothesize that the limited diffusivity of nanoshells in the liver, spleen, and pancreatic tumor is size-dependent, thus larger particles (>150 nm) would experience even greater diffusion limitations.

\section{Nanoparticle Accumulation in Liver, Spleen, and Pancreatic Tumor}

The average concentration of citrate gold nanoparticles was highest in the liver $(1.09 \pm 0.14$ nanoparticles per $\left.\mu \mathrm{m}^{2}\right)$, as compared to the spleen $\left(0.74 \pm 0.12\right.$ nanoparticles per $\left.\mu \mathrm{m}^{2}\right)$ and pancreatic tumor $\left(0.43 \pm 0.07\right.$ nanoparticles per $\left.\mu \mathrm{m}^{2}\right)$ (Figure 19A). In contrast, the average concentration of silica gold nanoshells was $0.43 \pm 0.07$ nanoparticles per $\mu \mathrm{m}^{2}$ in the liver, $0.30 \pm 0.06$ nanoparticles per $\mu \mathrm{m}^{2}$ in the spleen, and $0.20 \pm 0.04$ nanoparticles per $\mu \mathrm{m}^{2}$ in the pancreatic tumor (Figure 19A). Using the two-tailed student t-test, statistically significant differences between the accumulation of nanoparticles in each tissue are shown (all p-values $<0.05$ ). 

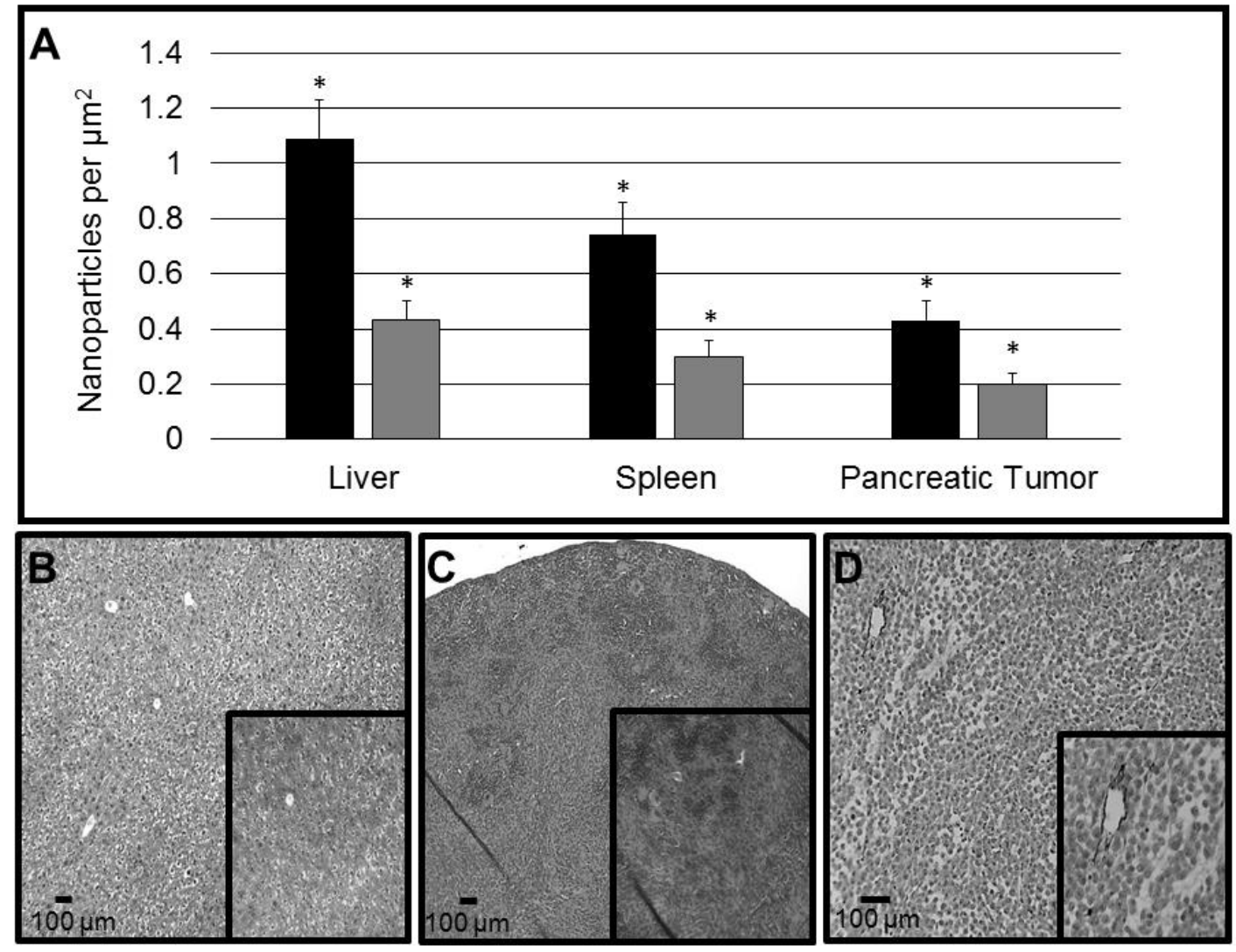

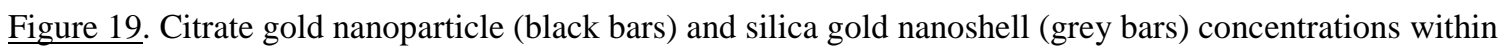
liver, spleen, and pancreatic tumor show differential uptake. (A) The liver exhibited highest concentration of either citrate gold nanoparticles or silica gold nanoshells, followed by the spleen and the pancreatic tumor. Statistically significant differences are signified by $(*)$ with a p-value $<0.05$, with statistical differences between each tissue type for both citrate gold nanoparticles and silica gold nanoshells. Error bars denote standard deviation. Hyperspectral imaging of nanoparticles in combination with dark field microscopy highlights the density of extravasation sites that may affect the uptake of nanoparticles within tissue. (B) The liver provides numerous extravasation sites. (C) The spleen also has numerous extravasation sites, albeit to a lesser extent compared to the liver. (D) The pancreatic solid tumor has fewer sites, leading to diminished nanoparticle uptake. Bars, $100 \mu \mathrm{m}$

Uptake of nanoparticles is a function of vasculature of an organ or tissue. The liver and spleen with similar patterns of highly vascularized tissue are expected to present a higher number of nanoparticle source points, while the pancreatic tumor with a more heterogeneous vascular pattern would have less. 
Dark-field tissue sections were compared to evaluate extravasation sites within the tissue (Figure 19B-D). Some regions of pancreatic tumor may contain large quantities of sprouting vessels due to sporadic angiogenesis, while other regions experience diminished vascular density with possibly few vessels supporting large volumes of tissue. This heterogeneity may result in tumor tissue becoming hypoxic and necrotic, with correspondingly restrained nanoparticle accumulation in these regions. 


\section{Discussion}

We synthesized PC-coated citrate gold nanoparticles and silica-gold nanoshells to study their uptake and diffusivity within tissue in vivo. PC is the most abundant phospholipid found in cellular membranes and thought to decrease possible immunogenicity [356]. The addition of PC was previously shown to significantly reduce associated cytotoxicity and particle aggregation, while enhancing passive targeting capabilities [357]. In previous work, we observed enhanced diffusivity of PC-coated nanoparticles in 3D cell cultures, with superior uptake and penetration in comparison to PEGylated nanoparticles [225]. PEG is currently the most widely employed FDA-approved polymeric platform used in the synthesis of medicinal nanoparticles for treating various cancers and related diseases, including Genexol-RM for metastatic breast cancer, Oncaspar for acute lymphoblastic leukemia, and Neulasta for chemotherapyassociated neutropenia [358]. While PEGylation is effective in enhancing drug delivery, multiple adverse effects have been associated with the use of PEG including possible immunogenicity, which is still debated [359]. The evaluation of PC-coated nanoparticles may longer term enable an alternative therapeutic option. We note that the only FDA-approved nanotherapeutic currently available for pancreatic cancer is Abraxane, an albumin-bound form of paclitaxel [360].

In this study, the formation of a bilayer membrane around nanoparticles was achieved by first adding hexadecanethiol, which displaced the outer coating of citrate and formed a hydrophobic nanoparticle (Figure 13). Through the addition of PC, the hydrophobic tails of the hexadecanethiol interact with the hydrocarbon chains of PC to form the membrane layer. The hydrophobic region between hexadecanethiol and PC can potentially be utilized for entrapping hydrophobic drugs, resulting in enhanced bioavailability. In addition, PC-coated silica-gold nanoshells exhibit wavelengths within the near infrared (nIR) region, making them potential candidates for photothermal ablation therapy as well as diagnostic

imaging through multispectral optoacoustic tomography (MSOT) [18, 361-363]. Recently, specific uptake of hybrid iron-oxide core gold-shell nanoparticles by pancreatic cells in photothermal therapy has been demonstrated. Cells exposed to nanoparticles and laser irradiation produced dose-dependent temperature increases and a reduction in cell proliferation, suggesting that photothermal ablation may be of use in the treatment of pancreatic cancer [364]. 
Detection of nanoparticles in tissue samples has been extensively accomplished using electron microscopy techniques (TEM, SEM, STEM) [365], yet newer methodologies present opportunities. Additional studies have utilized confocal microscopy to examine the translocation of nanoparticles in coculture cancer models [366]. While these models can detect nanoparticles, they lack the ability to confirm the presence of nanoparticles in tissue based upon spectral mapping. Hyperspectral imaging of nanoparticles in solution demonstrates the feasibility of determining the spatial location, agglomeration status, wavelength differentiation, and partial size determination [367]. Using hyperspectral imaging, nanoparticles can be further analyzed and characterized to determine properties such as surface modifications.

In this study, we used hyperspectral imaging to analyze the uptake and distribution of gold nanoparticles within tissue samples. We note that nanoparticle agglomeration can distort the results when attempting to assess nanoparticle concentrations within regions of interest. Consequently, numerical data gained from this method should be considered approximate and not absolute. Also, nanoparticle spectral profiles can be altered by factors including agglomeration status, backscatter from other sources, and other mechanisms. While the citrate gold nanoparticles exhibited similar spectra to those adapted from UV-Vis spectroscopy, the silica-gold nanoshells displayed a lower maxima (Figure 14). Such small changes are considered normal. Another common occurrence in detecting nanoparticles stems from the use of dense tissue samples, in which light scattering from different tissue components may hinder proper view. For this reason, spectral matching should be mapped for each image to computationally extract the nanoparticles, providing a clear depiction of which positive pixels are nanoparticles as compared to other tissue elements.

The spatial distribution of vessels within organs can cause some data distortion as vascular source points in mice reside within close proximity of each other [368]; nanoparticles extravasated from a particular source point may thus overlap with those extravasated from a nearby source. This was considered when counting the nanoparticles and was a reason to limit the distance of evaluation to $50 \mu \mathrm{m}$, which is the typical inter-vessel distance in the murine liver [369].

Recently, we have evaluated uptake and penetration of functionalized nanoparticles in avascular 3D cell cultures. Diffusion is both size and surface modification dependent, shown by analyzing the penetration of citrate gold nanoparticles and silica gold nanoshells (size difference $\sim 80 \mathrm{~nm}$ ) based on 
functionalization with hexadecanethiol (TL) and PC, or TL, PC, and HDL, and finding that coating with PC or HDL increased uptake and penetration [225]. The results herein show that PC-coated citrate gold nanoparticles exhibit a quasi-linear diffusion pattern in vivo similar to the 3D cell cultures (Figure 19A). In contrast, PC-coated silica gold nanoshells experienced a steeper decline in concentration from the nearest source point (Figure 19) compared that observed in 3D cell culture [225]. Further, the particle uptake observed within $10 \mu \mathrm{m}$ of the nearest source in the pancreatic tumor was on average $67 \%$ lower than observed with these same cells in vitro.

While 3D cell cultures mimic the morphological structure of tissue in vivo, tissue found within live subjects is more complex. This tissue is composed of a fully developed ECM and multiple cell types (including cells of the immune system) that are in contact with the blood supply and the lymphatic system, which may affect the deposition patterns and uptake of nanoparticles beyond the effects from 3D space. The extravasation in vivo is also expected to be hindered by interstitial fluid pressure within tumor tissue as has been shown in experimental as well as modeling studies [342, 345, 370]. The elevated concentration of nanoparticles in the liver and spleen highlights the sequestering by the RES (Figure 19A). Overall, the smaller citrate gold nanoparticles exhibited enhanced accumulation compared to the silica gold nanoshells. This difference can be primarily attributed to their smaller size. Nevertheless, the hypo-vascularization in the pancreatic tumor tissue can significantly hinder nanoparticle uptake regardless of size (Figure 19).

This study can be continued by examining the drug loading efficiency of the PC-coated nanoparticles. Hydrophobic drugs could be loaded into the hydrophobic region between the hexadecanethiol and PC, and thus facilitate their delivery through the hydrophilic tissue environment. To enhance local release, one would require the use of gold particles which absorb light in a region transparent to tissue, such as near infrared (nIR) absorbing gold nanoparticles (gold silica nanoshells, nanorods, or gold-sulfide aggregate nanoparticles). Drug release would then be mediated by heating of the surface (using the correct amount of energy via light dosing) to allow release of surface bound molecules. This method would minimize damage to surrounding tissue. Further, the nIR wavelength associated with the gold silica nanoshells in this study makes them potential candidates for photothermal ablation therapy. Citrate gold colloid based particles should not be used for thermal ablation as the wavelength of light $(\sim 540 \mathrm{~nm})$ used for activation will cause harm due to absorption of energy by the tissue. Nevertheless, citrate gold NPs 
may be easier for designing a layered system for drug transport modeling studies [225]. Finally, by analyzing diffusivity and distribution patterns of these nanoparticles in vitro and in vivo, mathematical modeling could be applied to help design and predict treatment outcomes [295, 296, 336, 370, 371]. 


\title{
CHAPTER IV
}

\section{RELEASE KINETICS OF CISPLATIN AND PACLITAXEL FROM TWO AND THREE LAYER NANOPARTICLES}

\author{
$\underline{\text { Background }}$ \\ Gold Nanoparticles for Cancer Therapy
}

Tumor chemotherapeutic response can be significantly affected by drug physiochemical properties, such as water solubility and bioavailability, as well as intrinsic and physiologic resistance by the tumor tissue itself. In order to enhance tumor response while minimizing systemic toxicity, a variety of drugs have been encapsulated in organic or inorganic nanoparticles, ranging in size from 1 to $1000 \mathrm{~nm}$. Gold nanoparticles, in particular, have been utilized as agents for drug delivery, thermal therapy, and in vivo imaging, and as radio-sensitizers for both pre-clinical and clinical purposes [372]. An early-phase clinical trial showed the effectiveness of 27-nm citrate gold nanoparticles functionalized with poly(ethylene)-glycol (PEG) while addition of tumor necrosis factor- $\alpha$ enhanced targeting of solid tumors and increased tumor toxicity [283].

\section{Cisplatin and Paclitaxel}

Two commonly utilized chemotherapeutics in cancer treatment are cisplatin and paclitaxel [373]. Cisplatin inhibits cell proliferation through multiple mechanisms, including: binding with DNA to form intra-stand adducts causing changes in DNA conformation, promoting mitochondrial damage leading to diminished energy production, altering cellular transport mechanisms, and decreasing ATPase activity within the cells $[148,374]$. Paclitaxel enhances tubulin polymerization to stable microtubules and stabilizes them against depolymerization, which results in mitotic arrest [375]. While both drugs are effective, they are known to possess adverse reaction profiles. Cisplatin induces renal toxicity caused by its activation within proximal and distal tubules, neurotoxicity by damaging Schwann cells of the myelin sheath, and tumor lysis syndrome (TLS), which results in abnormal metabolic and electrolyte profiles [148]. Paclitaxel 
has shown dose-limiting hematological toxicity (e.g. neutropenia) and sensory neurotoxicity, along with other adverse non-hematological toxicities including arthralgia, myalgia, and fluid retention [376]. In addition to adverse profiles, the poor water solubility and low bioavailability of paclitaxel has hampered its clinical use. The drug is administered in a solubilized form, Cremophor EL, to overcome minimal water solubility; while the castor oil used to solubilize the drug enhances bioavailability, it is known to induce histamine release resulting in hypersensitivity reactions in some patients $[15,377]$.

\section{Drug Release from Nanoparticle Systems}

The rate of drug release from nanoparticles is dependent upon the physiochemical properties of the drug, attachment strength between drug molecules and the nanoparticle surface, and surface modifications used in the synthesis process. Nanoparticles can be designed through alteration of these properties to exhibit drug release profiles specific for a targeted cancer to help optimize treatment response. The addition of surfactant PEG is known to escalate nanoparticle circulation time by one to two orders of magnitude compared to freely circulating drugs, providing additional time for nanoparticles to localize in the solid tumor tissue. Even without being actively targeted, studies have shown that PEG will increase nanoparticle accumulation in solid tumors due to the enhanced permeation and retention (EPR) effect caused by the irregular and leaky vasculature typically found in tumor tissue [378]. Through nanoparticle functionalization, drug release may be modulated to ensure sufficient time for nanoparticles to localize in the tumor (e.g., PEG-coated nanoparticles) or to release drug at specific locations (e.g., hypoxic regions) within the tumor microenvironment [379]. While drug release profiles can significantly alter response in situ, the surface modifications must also ensure that nanoparticles can successfully travel throughout systemic circulation to the tumor, extravasate from the intratumoral capillaries, and diffuse throughout the tissue to reach every malignant cell [380]. This can be a challenge as nanoparticles administered in vivo are often sequestered and removed from systemic circulation by the reticuloendothelial system (RES) [381].

The heterogeneous cell cycling patterns typically found in tumors ideally require nanoparticle accumulation with a sustained drug release. Paclitaxel-loaded gold nanoparticles have been extensively utilized for decreasing toxicity and lowering chemoresistance in various cancers [89, 382]. Highly stable PEG-coated gold nanoparticles loaded with paclitaxel have been synthesized, exhibiting a biphasic drug release pattern with an initial burst followed by a slower release over the next 120 hours [383]. Studies 
have shown that cisplatin-loaded gold nanoparticles show similar release patterns [384-388]. "Smartsensing" pH-sensitive nanoparticles have been developed that release cisplatin in specific environments, such as the acidic microenvironment of the tumor or within the cellular endosome once the nanoparticles have entered the cell [25]. Recently, controlled release of cisplatin from magnetic nanoparticles has been evaluated with the goal to minimize toxicity in non-cancerous tissues [389, 390].

\section{Purpose of Study}

In this study, we examine the release profiles of cisplatin and paclitaxel from novel two and three layer gold nanoparticles for the purpose of aiding the development of gold-based nanotherapeutics. Two layer gold nanoparticles were synthesized by adding hexadecanethiol and PC to the outside of gold cores. The addition of PC to the outer layer of TL creates a hydrophobic region, similar to the lipid bilayer found on liposomes, which can be utilized for loading hydrophobic drugs. For the three layer gold nanoparticles, HDL was added to the two layer nanoparticles for the purpose of improving liver targeting [225]. For both two and three layer gold nanoparticles, paclitaxel was loaded in the hydrophobic region between the TL and PC. Cisplatin was loaded through non-covalent interactions onto the outside of two or three layer gold nanoparticles. The release of drug was evaluated based upon particle surface modifications and drug physiochemical properties to determine the rate of drug release from nanoparticles. Kinetic models were evaluated to further assess the mechanisms of drug release, including: zero-order kinetic model, first-order kinetic model, simplified Higuchi model, and Korsmeyer-Peppas mode [391]. 
$\underline{\text { Methods }}$

Materials

$\mathrm{HAuCl}_{4}$ (Alfa Aesar, Ward Hill, MA, USA), trisodium citrate (Fisher Scientific, Waltham, MA, USA), 1-Hexadecanethiol (TL) (Sigma Aldrich), 100\% Ethanol (Decon Labs, King of Prussia, PA, USA), Chloroform (Sigma Aldrich), L-PC (Sigma Aldrich), HDL (Lee Biosolutions, St. Louis, MO, USA), Phosphate-Buffered Saline (PBS) (Life Technologies, Grand Island, NY), Cisplatin (Sigma Aldrich), Paclitaxel (Cayman Chemical, Ann Arbor, MI, USA), Acetonitrile (Sigma Aldrich), Trifluoroacetic acid (TFA) (Sigma Aldrich)

\section{Synthesis of Gold Nanoparticles}

Particles were synthesized using a method in which gold chloroauric acid is reduced by trisodium citrate as previously described [306]. In this process, $2.2-2.4 \mathrm{~mL} 1 \%$ weight/volume (wt/v) citrate is added to $200 \mathrm{~mL}$ of boiling $0.01 \% \mathrm{wt} / \mathrm{v} \mathrm{HAuCl}_{4}$, and the solution is allowed to continue boiling for 10 minutes to promote the reaction of sodium citrate to citric acid. Once the reaction is completed, the solution cools at room temperature before concentrated using a rotovapor (Buchi Rotovapor System, BÜCHI Labortechnik AG, Flawil, Switzerland) to $\sim 20 \mathrm{~mL}$. After the nanoparticles are concentrated, surface modifications were added as described below.

\section{Particle Functionalization}

The first layer applied to the citrate gold nanoparticles was 1-hexadecanethiol dissolved in ethanol. The thiol compound displaces the citrate bound to the core of the nanoparticles as thiol has a stronger binding affinity for the surface of gold in comparison to citrate, creating a hydrophobic nanoparticle, as the hydrocarbon chains of the thiol compound will point outward from the gold core. While stirring, $20 \mathrm{~mL}$ pure ethanol was placed in a beaker with $60 \mu \mathrm{L}$ 1-Hexadecanethiol being added secondly. The 1hexadecanethiol solution was added slowly to the nanoparticle solution over the next 10 minutes, while also agitating the sample by sonication. After addition of 1-Hexadecanethiol, the sample was agitated for two hours, and then placed for 12 hours on an orbital rocker (Boekel Scientific, Feasterville, PA, USA). The sample was spun down, and the pellet was washed twice and resuspended in chloroform. The second functionalization was the addition of the PC to the surface of nanoparticles. The stock solution was made by diluting PC in chloroform, and $100 \mu \mathrm{L}$ were added to the particles after the TL layer and allowed to set 
overnight on an orbital rocker. The solutions were transferred to glass tubes and the chloroform evaporated at ambient temperature. This process completed the two layer citrate gold nanoparticles containing gold core, TL, and PC. The three-layered nanoparticles were created by optimizing the ratio of HDL to particle optical density (1 mg HDL per 20 OD nanoparticle), and allowed to react overnight after two hours of agitation.

\section{Loading Nanoparticles with Drug}

Paclitaxel and cisplatin were loaded onto the nanoparticles in distinct regions to ensure optimal loading efficiency. Paclitaxel was loaded after the addition of 1-hexadecathiol and before the addition of PC. Briefly, after nanoparticles were placed on an orbital shaker for 12 hours, the solution was removed. After nanoparticles were resuspended in $9 \mathrm{~mL}$ chloroform, an addition $1 \mathrm{~mL}$ of chloroform containing $5 \mathrm{mg}$ paclitaxel was added to the solution. Nanoparticles were agitated for two hours using sonication before the solution was placed on an orbital rocker for six hours. The solution was further modified to add the second layer of PC to the surface of the nanoparticles. While paclitaxel was loaded into the hydrophobic region created between the TL and PC layer, cisplatin was loaded at two different areas dependent upon the layering. For the two layer citrate gold nanoparticles, cisplatin was added after the addition of PC. This was done by transferring the solutions to glass tubes and the chloroform evaporated at ambient temperature. Next, the nanoparticles were resuspended in $10 \mathrm{~mL}$ ultrapure $\mathrm{H}_{2} \mathrm{O}$ (Purelab Ultra, Elga Labwater, UK) containing $7.5 \mathrm{mg}$ cisplatin. For the three layer citrate gold nanoparticles, cisplatin was added after the addition of HDL by synthesizing the particles as described above, yet after HDL was added to the particles and allowed to react for two hours, the solution was removed, and $7.5 \mathrm{mg}$ cisplatin was added. Excess chemotherapeutic was removed from the solution by centrifuging the particles at $7000 \mathrm{rpm}$ for 25 minutes, removing the supernatant, and re-suspending the particles in the corresponding solvent. Washing was performed twice.

\section{Nanoparticle Characterization}

Nanoparticle identity was verified as follows: (1) Maximum absorption wavelengths were obtained using the Varian Cary 50 Bio Ultraviolet-Visible (UV-Vis) Spectrometer (McKinley Scientific); (2) size and zeta potential measurements were obtained using the ZetaSizer Nanoseries ZS90 (Malvern Instruments, Worcestshire, UK); (3) DLS (dynamic light scattering, also known as Photon Correlation 
Spectroscopy) to determine hydrodynamic size in solution based upon Brownian motion; (4) shape and size were determined using a Zeiss Supra 35VP (Carl Zeiss, Oberkochen, Germany) SEM; (5) presence of lipids on the particle cores was confirmed using a FTIR instrument (Perkin Elmer Spectrum BX; Perkin Elmer, Waltham, MA, USA) and through visual analysis using the SEM.

\section{In Vitro Drug Release Studies}

In vitro drug release studies were carried out using dialysis tubing cellulose membrane with an average flat width of $25 \mathrm{~mm}$ and 12,000 MW cutoff (Fisher Scientific, Waltham, MA, USA). The prepared drug-loaded nanoparticles were added to dialysis tubes and subject to dialysis by submerging the tubing into a beaker containing $500 \mathrm{~mL} 1 \mathrm{X}$ PBS at $\mathrm{pH}$ 7.4. The solution was agitated continuously throughout the release study using a magnetic stirrer at room temperature covered with Parafilm to ensure no evaporation would take place during the study. At established time intervals, $3 \mathrm{~mL}$ samples of PBS containing drug were removed and replaced with fresh buffer to ensure a constant volume. The amount of drug in each sample was determined using HPLC. Cumulative drug release versus time was expressed by the following equation (Eq. 1):

Cumulative Drug Release (\%) $=\frac{[\text { Drug }]_{t}}{[\text { Drug }]_{\text {total }}} \times 100 \quad$ Eq. (1)

where $[\text { Drug }]_{t}$ refers to the concentration of drug release at time $t$ and $[\text { Drug }]_{\text {total }}$ is the total amount of drug loaded onto the nanoparticles.

\section{Drug Detection using HPLC}

Samples of paclitaxel and cisplatin were analyzed using a Waters Alliance e2695 HPLC equipped with a Waters 2998 photodiode array UV/Vis detector and a $\mu$ RPC C2/C18 ST 4.6/100 column (GE Healthcare, catalog number 17-5057-01). Initial injection conditions were $100 \%$ water/0.1\% TFA immediately followed upon injection by 5 minutes with $100 \%$ water/0.1\% TFA, 35 minutes of linear gradient to $100 \%$ acetonitrile/ $0.1 \%$ TFA, 5 minutes at $100 \%$ acetonitrile, followed by a return to $100 \%$ water/0.1\% TFA to prepare the column for the next run. Total run time was 55 minutes. The flow rate was $0.5 \mathrm{ml} / \mathrm{min}$. Spectrophotometric data were collected from 200 to $800 \mathrm{~nm}$. The baseline for each run was monitored at $260 \mathrm{~nm}$ and $280 \mathrm{~nm}$. A standard calibration curve was created for paclitaxel $(0.01 \mu \mathrm{M}$ to 10 $\mu \mathrm{M}$ plus a blank sample) and cisplatin ( $2.5 \mu \mathrm{M}$ to $\mu 500 \mathrm{M}$ plus a blank sample) by injection of pure compounds dissolved in water or buffer. 
The peak corresponding to paclitaxel was integrated at $230 \mathrm{~nm}$ to minimize overlap of peaks belonging to interfering compounds and to maximize peak area. Cisplatin was integrated at $380 \mathrm{~nm}$ for the same reasons. After elution, peaks were integrated using Waters Empower software. A calibration curve was generated by plotting peak area vs. concentration using Microsoft Excel. Analytical samples of each compound were then compared to the standard curve to determine their approximate concentration.

\section{Determination of Drug Encapsulation Efficiency and Drug Loading Efficiency}

Drug incorporation efficiency (I.E.) (\%) was expressed as the percentage of drug in the produced nanoparticles with respect to the initial amount of drug that was used for synthesizing the nanoparticles [392]. This calculation was determined using HPLC as described above in conjunction with the following equation (Eq. 2):
I.E. $(\%)=\frac{\text { Amount of Drug in Nanoparticles }(\mathrm{mg})}{\text { Intial Amount of Drug }(\mathrm{mg})} \times 100$
Eq. (2)

\section{Mechanism of Drug Release}

To assess the mechanism of drug release, in vitro release patterns were analyzed using four kinetic models: zero-order kinetic model, first-order kinetic model, simplified Higuchi model, and KorsmeyerPeppas model. The zero order model is associated with drug dissolution that is independent of drug concentration (Eq. 3) [393]:

$Q_{t}=Q_{0}+k_{0} t$

where $Q_{t}$ is the amount of drug dissolved in time $t, Q_{0}$ is the initial amount of drug in solution, and $k_{0}$ describes the zero-order rate constant. The first order model describes drug release that is concentrationdependent (Eq. 4) [393]:

$\frac{d C}{d t}=-k C$

where $C$ refers to drug concentration and $k$ is the first order rate constant. This equation can also be expressed as (Eq. 5):

$\log C=\log C_{0}-\frac{k t}{2.303}$

where $C_{0}$ corresponds to the initial concentration of drug. The simplified Higuchi model utilizes the following equation to describe drug release from matrix and polymeric systems (Eq. 6) [394]: 
$\frac{M_{t}}{M_{\infty}}=k \sqrt{t}$

Eq. (6)

where $\left(M_{t} / M_{\infty}\right)$ is the cumulative amount of drug released at time $t$, and $k$ is the Higuchi constant based upon the formulation of the system. The Korsmeyer-Peppas model describes drug release from matrix and polymeric systems through the following equation (Eq. 7) [395]:

$\frac{M_{t}}{M_{\infty}}=k^{\prime} t^{n}$

Eq. (7)

where $\left(M_{t} / M_{\infty}\right)$ is the cumulative amount of drug released at time $t, k$ ' is the kinetic constant, and $n$ is the exponent that describes a particular diffusion mechanism.

The first $60 \%$ of drug release is typically sufficient for determining the best fit model of drug release [396]. For each model, a graph was constructed using Microsoft Excel from which the rate constant and correlation values were obtained by applying a linear regression fit. The zero-order kinetic model was obtained by plotting cumulative $\%$ drug release vs. time. The first-order kinetic model was analyzed by plotting log cumulative $\%$ of drug remaining vs. time. The Higuchi model was evaluated by plotting cumulative \% drug release vs. square root of time, while the Korsmeyer-Peppas model was analyzed by plotting log cumulative \% drug release vs. log time. 


\section{$\underline{\text { Results }}$}

\section{Nanoparticle Synthesis and Characterization}

We have previously characterized the diffusivity and transport of two and three layer gold nanoparticles in 3D cell cultures and in vivo tissue in Chapter 2, finding that they performed better than PEG-coated versions. Here, we examine the in vitro release profiles of cisplatin and paclitaxel from such nanoparticle formulations. We evaluate nanoparticles functionalized with TL and PC for the development of an inner hydrophobic region with a surrounding hydrophilic exterior, or TL, PC and HDL as three layered gold nanoparticles (Figure 20). To ensure proper synthesis and surface functionalization, nanoparticles were characterized through UV-Vis (ultraviolet-visible) spectroscopy to determine maxima absorbance, SEM for morphological and size analysis, DLS (dynamic light scattering) to determine hydrodynamic size in solution based upon Brownian motion, zeta potential to determine surface charge, and FTIR analysis to ensure the presence of surface modifications.

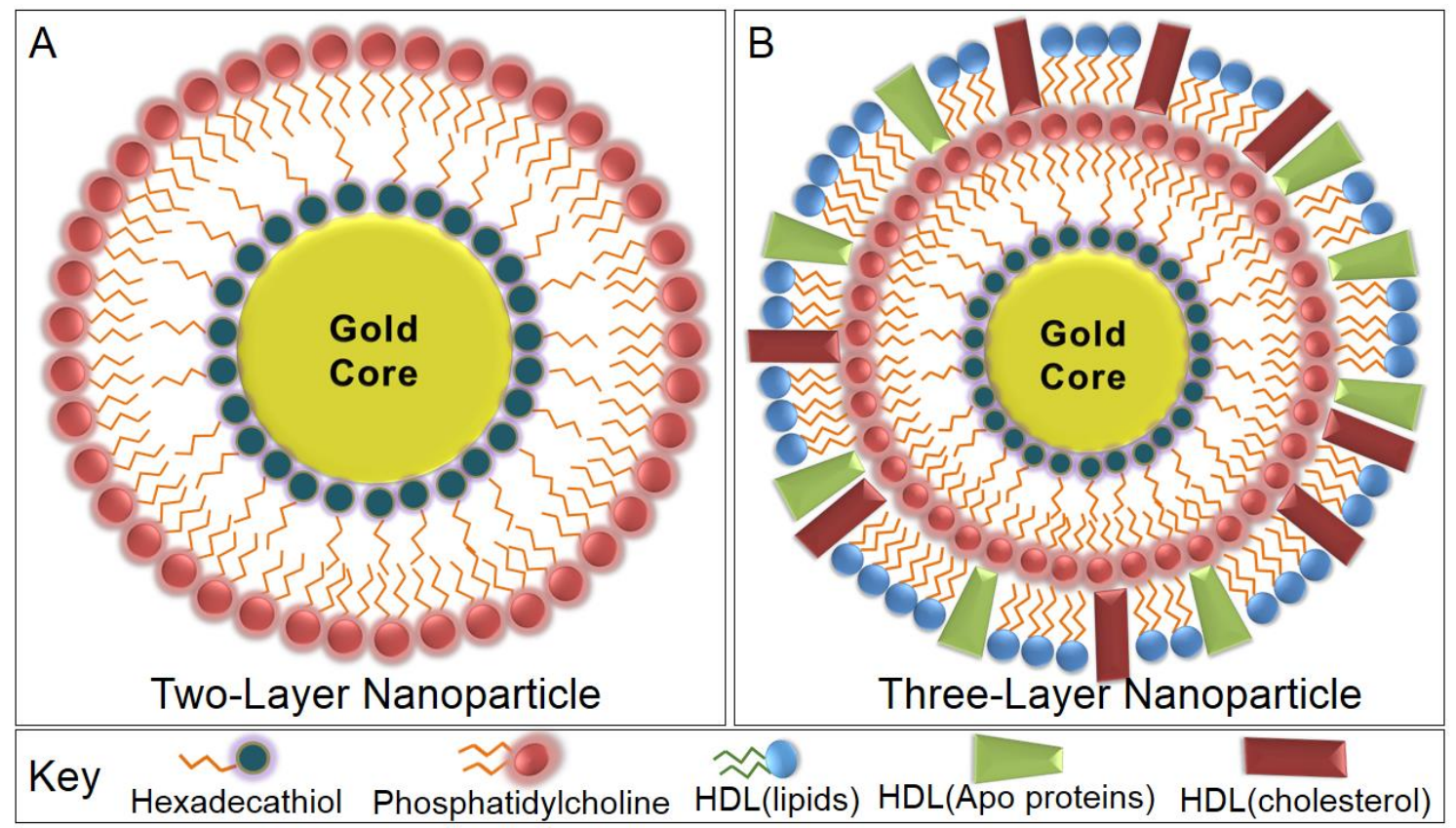

Figure 20. Nanoparticle Illustration of Synthesis and Functionalization. Nanoparticles were synthesized with either two or three layers, in which a lipid containing a hexadecanethiol (TL) head group was applied to the gold surface. This displaced the citrate stabilizer, forming a hydrophobic nanoparticle. The addition of PC to the solution promoted water solubility as the hydrophobic tails of PC bound the tails of TL. The 
two layer gold nanoparticles (A) were compared to three layer nanoparticles (B), in which HDL was further added to alter the in vivo reactivity, drug release profile, and enhancement of tumor targeting.

Optical measurements were performed through UV-Vis spectroscopy and offer information regarding nanoparticle size, shape, and agglomeration status. The spectra of two layer gold nanoparticles exhibited a maximum absorbance peak at $540 \mathrm{~nm}$, while three layer gold nanoparticles displayed a similar spectrum with a maximum absorbance of $541 \mathrm{~nm}$ (Figure 21A). This particular wavelength near 534-545 $\mathrm{nm}$ is characteristic for nanoparticles with a diameter $50-70 \mathrm{~nm}$, as nanoparticle size is proportional to maximum absorbance with larger nanoparticles shifting maximum absorbance values to higher spectral wavelengths [313]. Visual determination of nanoparticle size was accomplished using SEM, showing that two layer gold nanoparticles had an average size of $47.1 \pm 12.6 \mathrm{~nm}$, while three layer gold nanoparticles were $33 \%$ larger with an average size of $62.8 \pm 14.9$ (Figure 21B). The size difference can be attributed to the addition of HDL to the surface of PC. 

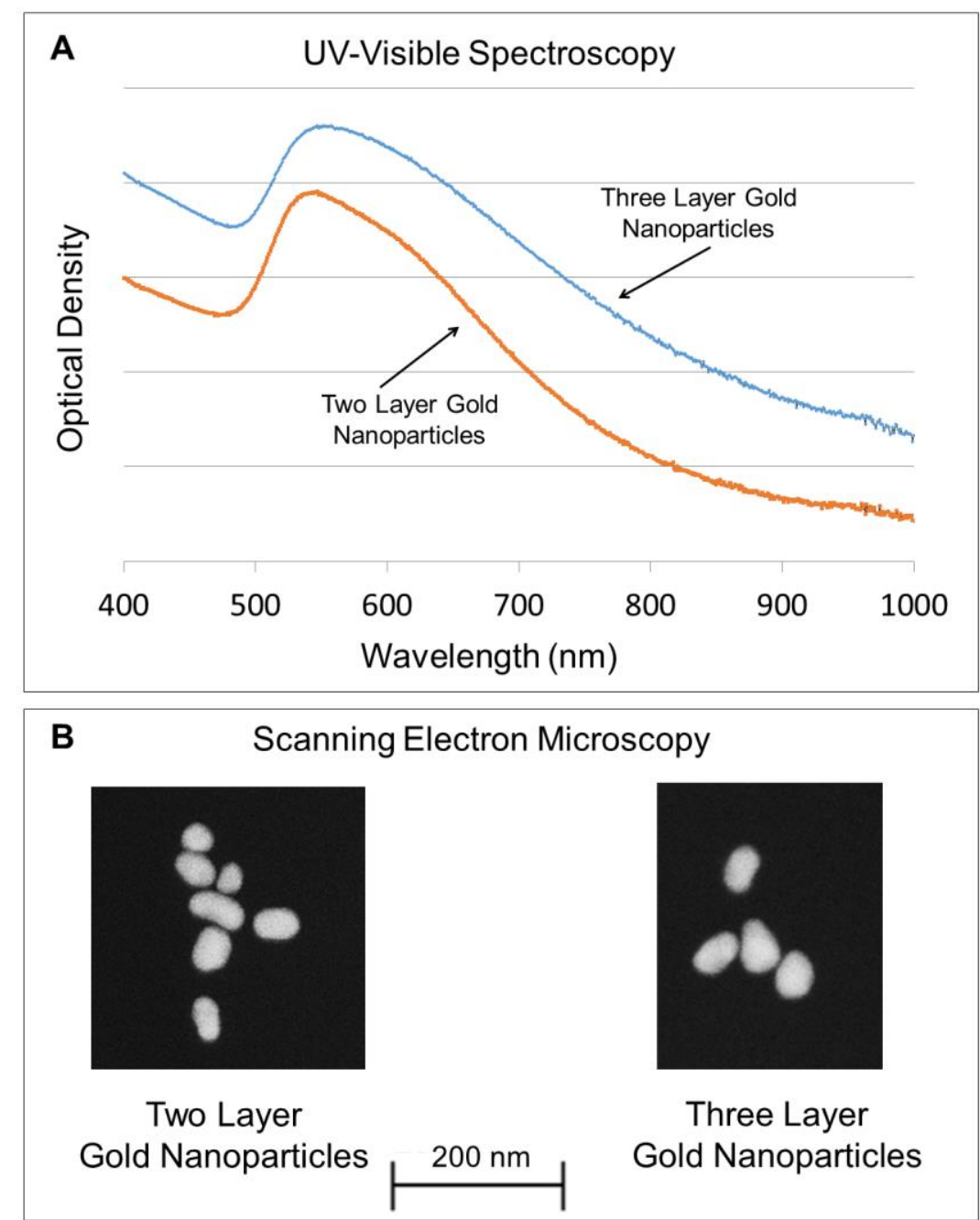

Figure 21. UV-Spectroscopy and Zeta Potential Analysis of Gold Nanoparticles. Gold nanoparticles were characterized using UV-Vis spectroscopy to determine the maximum absorbance wavelength and with scanning electron microscopy (SEM) for size analysis. (A) The maximum absorbance of two and three layer gold nanoparticles was $542 \mathrm{~nm}$ and $537 \mathrm{~nm}$, respectively. Nanoparticles optical density does not correspond to actual O.D. as both spectra were modified to fit graph. (B) SEM showed the size of two layer gold nanoparticles at $47.1 \pm 12.6 \mathrm{~nm}$ and three layer gold nanoparticles at $62.8 \pm 14.9 \mathrm{~nm}$.

DLS establishes the hydrodynamic size of nanoparticles in solution by considering Brownian motion. As nanoparticles travel throughout solution, a thin electric dipole layer of solvent adheres to their surface, which in turn can hinder or assist nanoparticles in reaching their target destination. Figure 22 reveals the hydrodynamic size for two and three layer gold nanoparticles at $74.91 \pm 13.3 \mathrm{~nm}$ and $85.26 \pm$ 
$18.7 \mathrm{~nm}$, respectively (Table 2). The surface charge of two and three layer gold nanoparticles, determined through zeta potential analysis as illustrated in Figure 20, shows that HDL-coated nanoparticles (-2 mV) were more neutrally charged in comparison to anionic PC-coated gold nanoparticles at -20 mV (Table 2). For comparison, un-coated gold nanoparticles possessed a zeta potential near $-40 \mathrm{mV}$, while thiol coated nanoparticles were approximately $-30 \mathrm{mV}$.

\section{Two Layer Gold Nanoparticles Three Layer Gold Nanoparticles}

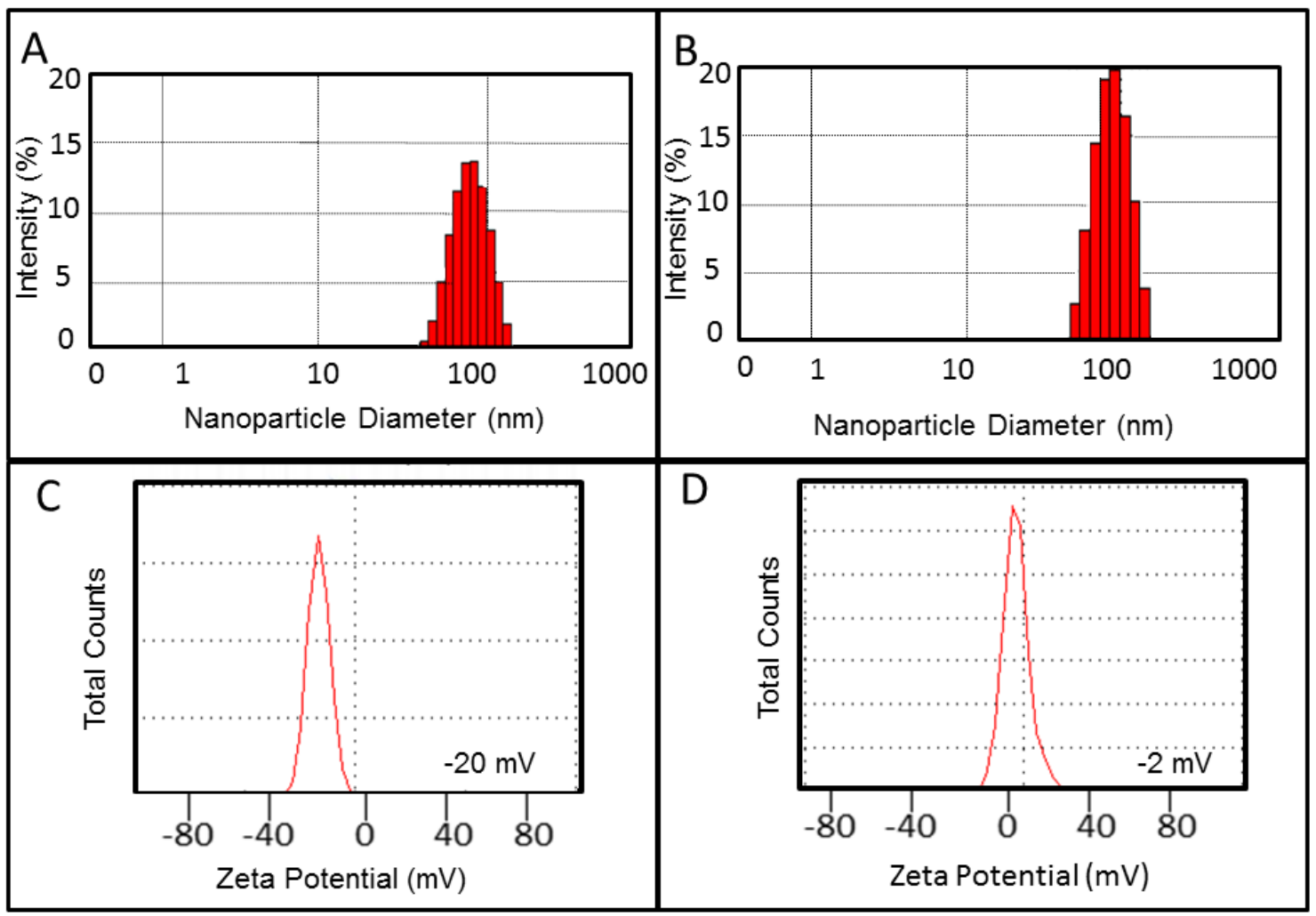

Figure 22. Hydrodynamic Size of Gold Nanoparticles in Solution. Gold nanoparticles were characterized using dynamic light scattering (DLS) to determine hydrodynamic size in solution and with zeta potential to determine surface charge. (A) The hydrodynamic size of two and three layer gold nanoparticles was determined to be $74.91 \pm 13.3 \mathrm{~nm}$ and $85.26 \pm 18.7 \mathrm{~nm}$, respectively. (B) Two layer gold nanoparticles exhibited an anionic charge of $-20 \mathrm{mV}$, while three layer gold nanoparticles were more neutrally charged at $-2 \mathrm{mV}$.

Table 2

Characterization Results of Two and Three Layer Gold Nanoparticles 


\begin{tabular}{|c|c|c|c|c|c|}
\hline Nanoparticle & Surface Modification & Max A bsorbance (nm) & SEM Size (nm) & DL S Size (nm) & Zeta Potential (mV) \\
\hline Citrate Gold & TL-PC & 540 & $47.1(12.6)$ & $74.91(13.3)$ & -20 \\
Citrate Gold & TL-PC-HDL & 541 & $62.8(14.9)$ & $85.26(18.7)$ & -2 \\
\hline
\end{tabular}

FTIR was employed to confirm the presence of surface modifications (Figure 23). Spectra obtained from two and three layer gold nanoparticles were compared with spectra of pure PC [397] and HDL [333]. Two layer gold nanoparticles functionalized with TL and PC exhibited several signature peaks that confirmed the presence of TL and PC onto the gold core. Signature peaks included $\mathrm{PO}_{3-}^{4}$ group vibrations between $\sim 850-1000 \mathrm{~cm}^{-1}$, a C-O-C stretch $\sim 1100 \mathrm{~cm}^{-1}$, a $\left[\left(-\mathrm{CH}_{2}\right)_{\mathrm{n}}\right]$ rocking vibration $\sim 720 \mathrm{~cm}^{-}$ 1, both asymmetric and symmetric $-\mathrm{CH}_{2}\left(2880 \mathrm{~cm}^{-1}\right)$ and $-\mathrm{CH}_{3}\left(2950 \mathrm{~cm}^{-1}\right)$ stretch and vibration, and a $\mathrm{CH}_{2}$ stretching and scissoring at 1375 and $1470 \mathrm{~cm}^{-1}$, respectively. Slight differences in the spectra can be attributed to other chemicals used in the synthesis of the layered nanoparticles, including TL and colloidal gold. For HDL-coated nanoparticles, the asymmetric and symmetric $-\mathrm{CH}_{2}\left(2880 \mathrm{~cm}^{-1}\right)$ and $-\mathrm{CH}_{3}(2950$ $\mathrm{cm}^{-1}$ ) stretch and vibration occur along with $\mathrm{C}=\mathrm{O}$ from the lipid ester $\sim 1700-1800 \mathrm{~cm}^{-1}$, along with amide bond stretches between $1500-1700 \mathrm{~cm}^{-1}$, and a phospholipid $\mathrm{P}=\mathrm{O}_{2}$ stretch $\sim 1250 \mathrm{~cm}^{-1}$. As these nanoparticles were also coated with TL and PC, distinct bands from both TL and PC were expected to be present in the spectra of the three layer formulation. 


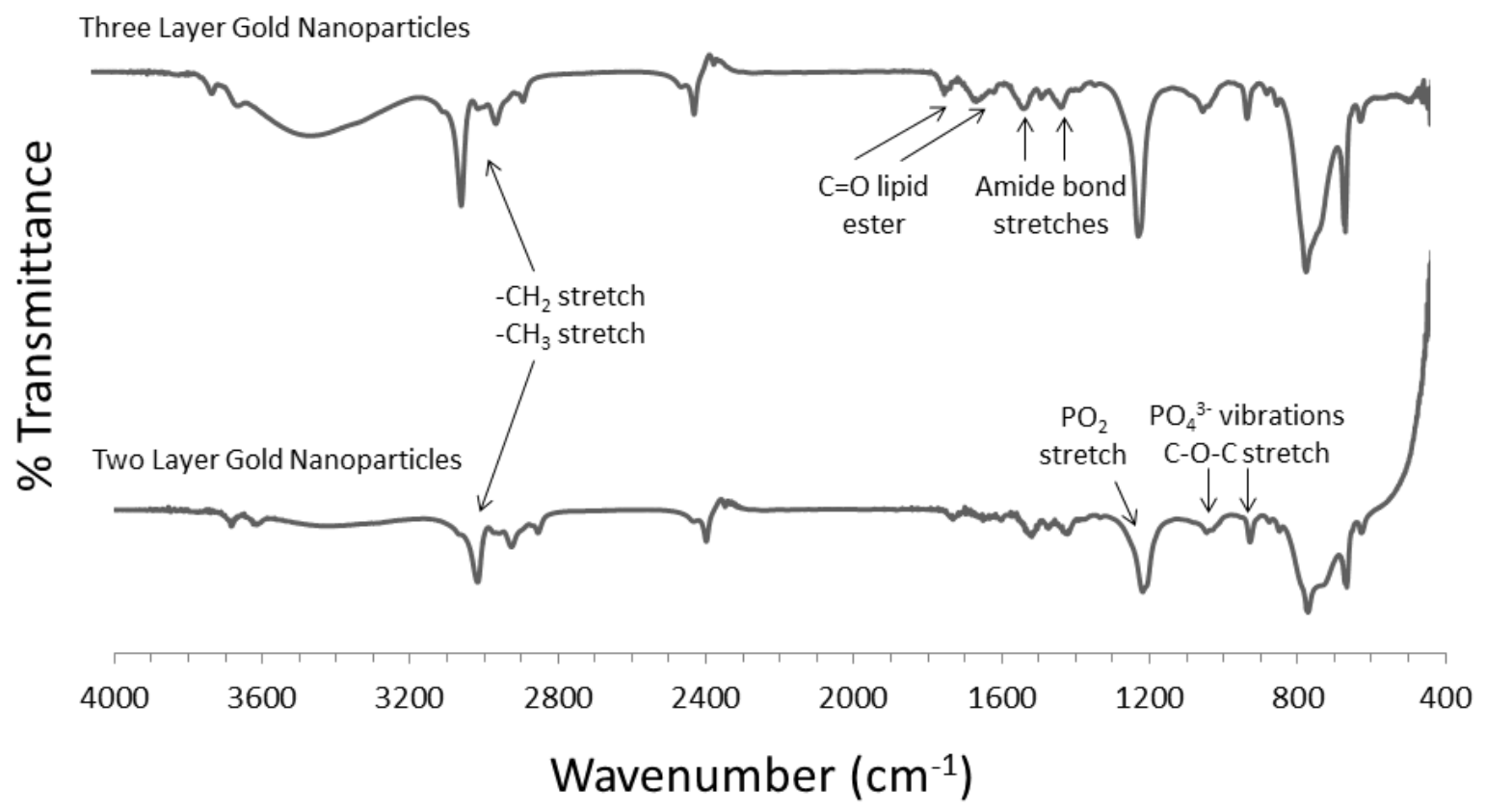

Figure 23. Surface Modification Analysis using FTIR. Gold nanoparticle surface modifications were confirmed using Fourier Transform Infrared Spectroscopy (FTIR). The peaks were matched with those of pure PC and HDL. The PC-coated two layer gold nanoparticles exhibited multiple peaks that were used for conformation, including a $\left[\left(-\mathrm{CH}_{2}\right)_{\mathrm{n}}\right]$ rocking vibration $\sim 720 \mathrm{~cm}^{-1}$, a $\mathrm{PO}_{4}{ }^{3-}$ group vibration between 820 $1000 \mathrm{~cm}^{-1}$, C-O-C stretch $\sim 1100 \mathrm{~cm}^{-1},-\mathrm{CH}_{2}$ stretching and scissoring (1375 and $\left.1470 \mathrm{~cm}^{-1}\right)$. The HDLcoated three layer gold nanoparticles exhibited several peaks including: asymmetric and symmetric $-\mathrm{CH}_{2}$ $\left(2880 \mathrm{~cm}^{-1}\right),-\mathrm{CH}_{3}\left(2950 \mathrm{~cm}^{-1}\right)$ stretch and vibration, $\mathrm{C}=\mathrm{O}$ from the lipid ester between $1700-1800 \mathrm{~cm}^{-1}$, amide bond stretches between $1500-1700 \mathrm{~cm}^{-1}$ and a phospholipid $\mathrm{P}=\mathrm{O}_{2}$ stretch $\sim 1250 \mathrm{~cm}^{-1}$.

\section{Drug Release from Two and Three Layered Gold Nanoparticles}

Both two and three layer gold nanoparticles were loaded with either cisplatin or paclitaxel to evaluate the effect that the surface modifications may have on hydrophilic and hydrophobic drug release kinetics. The cumulative percent of drug release was plotted against time to analyze the drug release kinetics for each case (Figure 24). For cisplatin-loaded two layer gold nanoparticles, an initial burst of 35.7 $\pm 2.3 \%$ was observed during the first 5 hours, followed by a steady release for the next 14 days (336 hours), with $64.0 \pm 2.4 \%$ of loaded cisplatin released (Figure 24A). Three layer gold nanoparticles loaded with cisplatin also showed an initial burst of $68.4 \pm 1.0 \%$, followed by a steady profile with $98.3 \pm 2.6 \%$ of 
loaded drug released at the end of 14 days (Figure 24B). Drug release within the first 24 hours was plotted separately to highlight the initial burst followed by the switch to a more linear profile (Figure 25).

Paclitaxel release from two layer gold nanoparticles showed a linear profile with only $22.3 \pm 1.5 \%$ of loaded drug being released at the end of 14 days, indicating that nearly $78 \%$ of entrapped drug was still attached to the nanoparticles (Figure 24C). In contrast, the three layer formulation effectively released 97.8 $\pm 2.3 \%$ of encapsulated drug by day 14 (Figure 24D). The first 24 hours were also plotted separately to highlight the initial release (Figure 25).
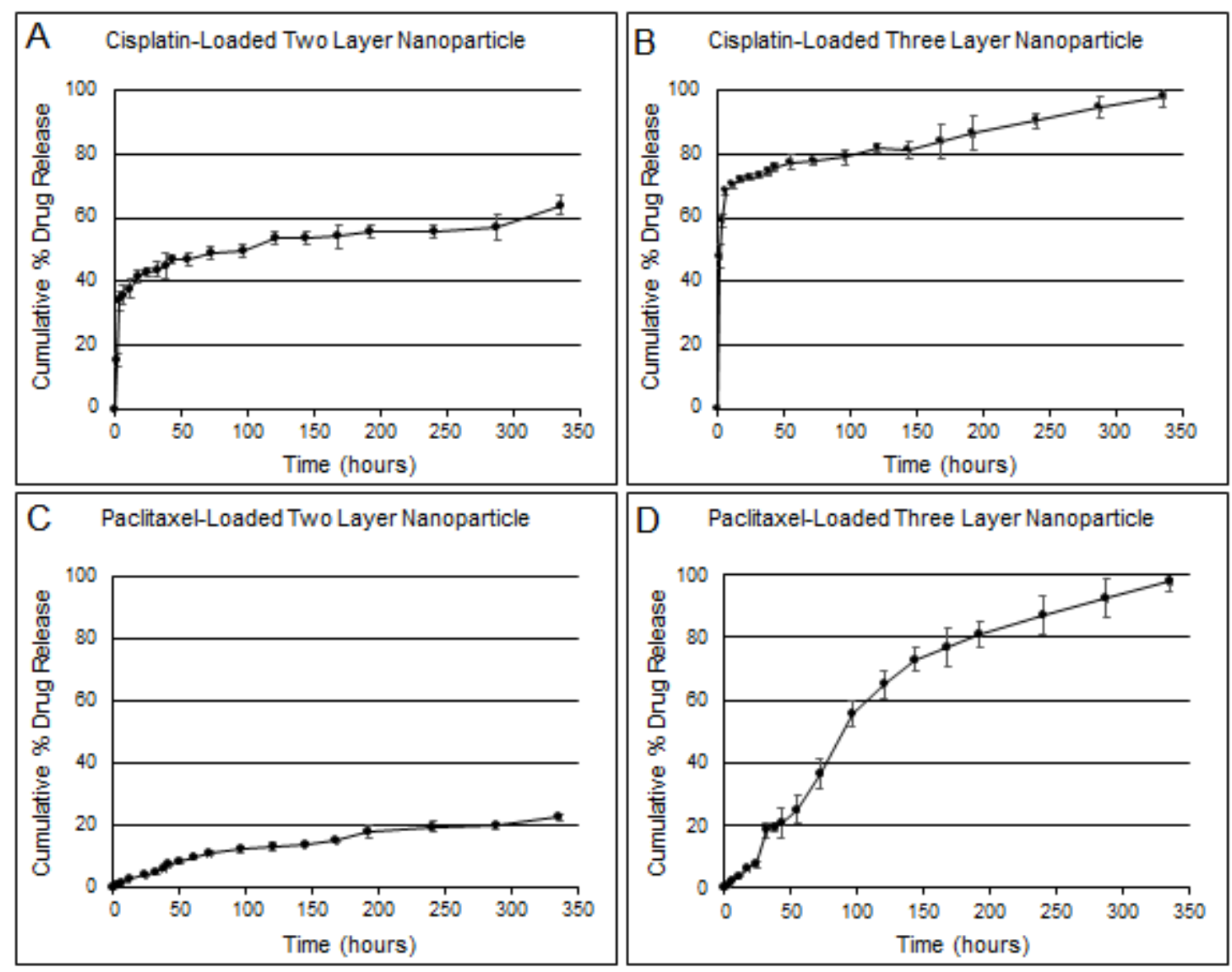

Figure 24. Drug Release from Functionalized Two and Three Layer Gold Nanoparticles. Hydrophilic and hydrophobic drug release profiles from gold nanoparticles coated with PC and TL (two layer), or PC, TL, and HDL (three layer). (A) Cisplatin-loaded two layer gold nanoparticles exhibited a burst during the first 5 hours, with $\sim 35 \%$ of drug being released. A steady release followed over the next 14 days. (B) Cisplatinloaded three layer gold nanoparticles also experienced an initial burst with $~ 70 \%$ of encapsulated drug being released within the first 5 hours. Drug release then became steady for the next 14 days. (C) Paclitaxel 
release from two layer gold nanoparticles was steady with only $~ 20 \%$ of encapsulated paclitaxel released during the 14 days. (D) Almost 100\% of paclitaxel encapsulated within three layer gold nanoparticles was released by 14. Error bars represent standard deviation $(n=3)$.
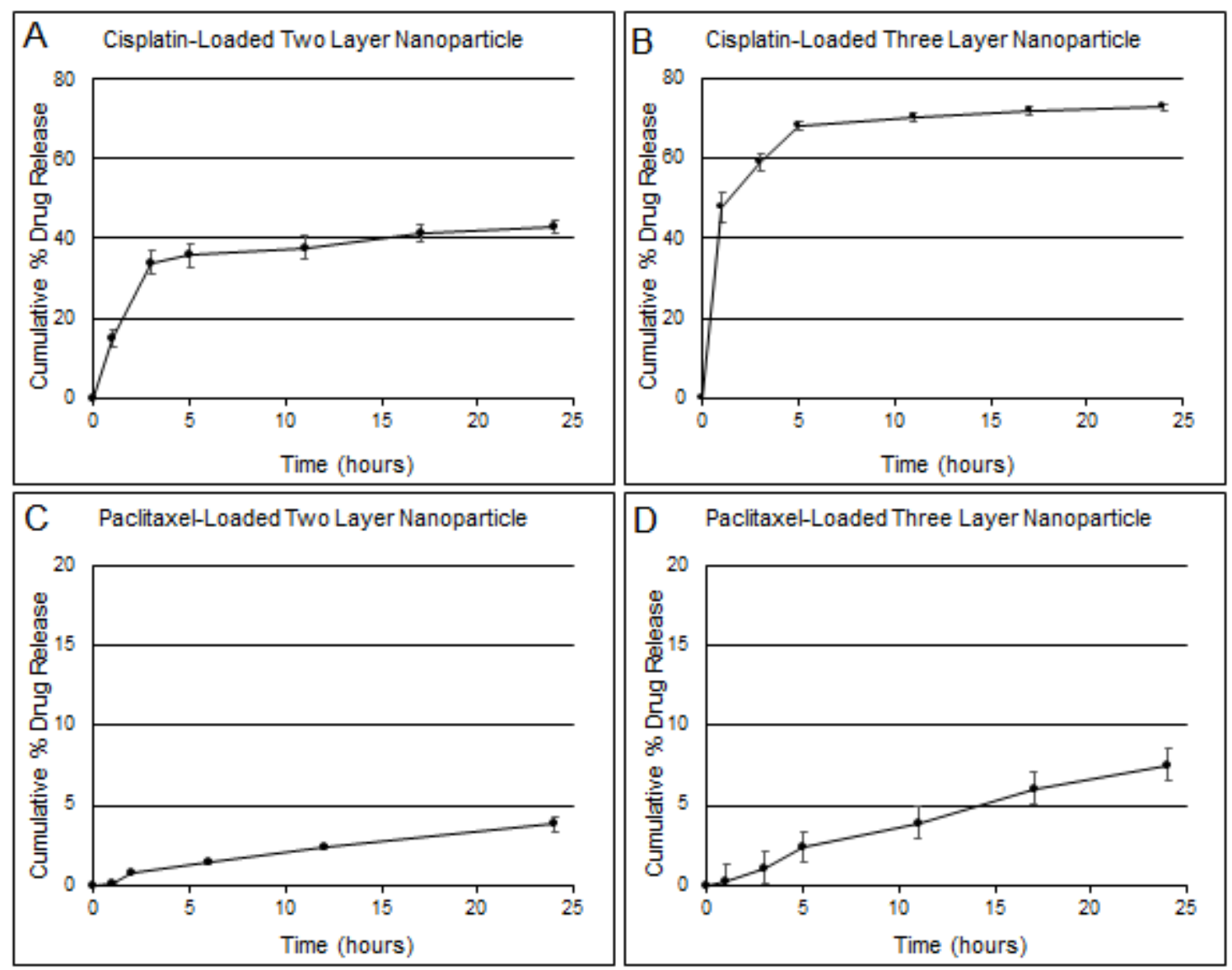

Figure 25. First 24 Hours of Drug Release from Gold Nanoparticles. Graphs highlighting drug release from two- and three-layer gold nanoparticles during the first 24 hours are included. Additional graphs illustrating model fitting to release curves are also shown.

The amount of drug loaded onto the nanoparticles was determined indirectly by measuring the amount of drug that did not load (Table 3). For two layer gold nanoparticles, $68.4 \pm 7.1 \%$ of cisplatin and $78.9 \pm 4.9 \%$ of paclitaxel were effectively loaded. For the three layer formulation, higher drug incorporation efficiencies were obtained with $99.1 \pm 0.7 \%$ of cisplatin and $99.4 \pm 0.4 \%$ of paclitaxel 
loaded. Nearly $100 \%$ of paclitaxel became encapsulated, suggesting that even higher drug concentrations may be possible.

\section{Table 3}

Drug Incorporation Efficiency of Two and Three Layer Gold Nanoparticles

\begin{tabular}{|c|c|c|c|}
\hline Nanoparticle & Surface Modification & Incorporated Drug & Drug Incorporation Efficiency (\%) \\
\hline Citrate Gold & TL-PC & Cisplatin & $68.4 \pm 7.1$ \\
Citrate Gold & TL-PC-HDL & Cisplatin & $78.9 \pm 4.9$ \\
\hline Citrate Gold & TL-PC & Paclitaxel & $99.1 \pm 0.7$ \\
\hline Citrate Gold & TL-PC-HDL & Paclitaxel & $99.4 \pm 0.4$ \\
\hline
\end{tabular}

\section{Kinetic Models for Drug Release}

Mathematical models may be useful to evaluate the kinetics and mechanism of drug release from nanoparticles. The release curves from Figure 24 were fitted to four distinct models to determine which one exhibited the highest correlation with experimental results (Table 4). Hydrophobic drug (paclitaxel) release from three layer gold nanoparticles exhibited high correlation with the zero-order kinetic model and the Korsmeyer-Peppas models, both with $\mathrm{R}^{2}>0.98$ (Figure 26). Release of paclitaxel from the two layer gold nanoparticles also showed high correlation with the simplified Higuchi model $\left(\mathrm{R}^{2}=0.9862\right)$, possibly due to the profile curve denoting an early stage of release since only $22.3 \pm 1.5 \%$ of paclitaxel was unloaded by day 14. Both two and three layer gold nanoparticles loaded with hydrophilic drug (cisplatin) correlated best with the Korsmeyer-Peppas model with $\mathrm{R}^{2}>0.98$. Both the Higuchi and Korsmeyer-Peppas models are typically used to describe drug release from degrading matrix and polymeric systems [398]. Although in this study the particles were metallic, the results suggest that aggregate release of drug from multiple particles confined within a dialysis bag may be modeled similar to a polymeric system which undergoes degradation.

\section{Table 4}

Rate Constants and Correlation Coefficients Obtained from Modeling Drug Release from Two and Three Layer Gold Nanoparticles through the following: zero-order kinetic model, first-order kinetic model, simplified Higuchi model, and Korsmeyer-Peppas model. 


\begin{tabular}{|c|c|c|c|c|c|c|c|c|c|}
\hline \multirow[t]{2}{*}{ Surface Modification } & \multirow[t]{2}{*}{ Incorporated Drug } & \multicolumn{2}{|c|}{ Zero-Order } & \multicolumn{2}{|c|}{ First-Order } & \multicolumn{2}{|c|}{ Higuchi } & \multicolumn{2}{|c|}{ Korsmeyer-Peppas } \\
\hline & & $\mathrm{k}$ & $r^{2}$ & $\mathrm{k}$ & $r^{2}$ & $\mathrm{k}$ & $r^{2}$ & $\mathrm{n}$ & $r^{2}$ \\
\hline TL-PC & Cisplatin & 0.0725 & 0.8445 & 0.0008 & 0.5875 & 1.8554 & 0.8061 & 0.1248 & 0.9811 \\
\hline TL-PC-HDL & Cisplatin & 11.699 & 0.7284 & 0.089 & 0.8478 & 30.162 & 0.9287 & 0.2716 & 0.9897 \\
\hline TL-PC & Paclitaxel & 0.0668 & 0.9127 & 0.0003 & 0.9313 & 1.294 & 0.9862 & 0.6787 & 0.9846 \\
\hline TL-PC-HDL & Paclitaxel & 0.5589 & 0.9869 & 0.0037 & 0.9589 & 5.8756 & 0.8766 & 1.1348 & 0.9891 \\
\hline
\end{tabular}
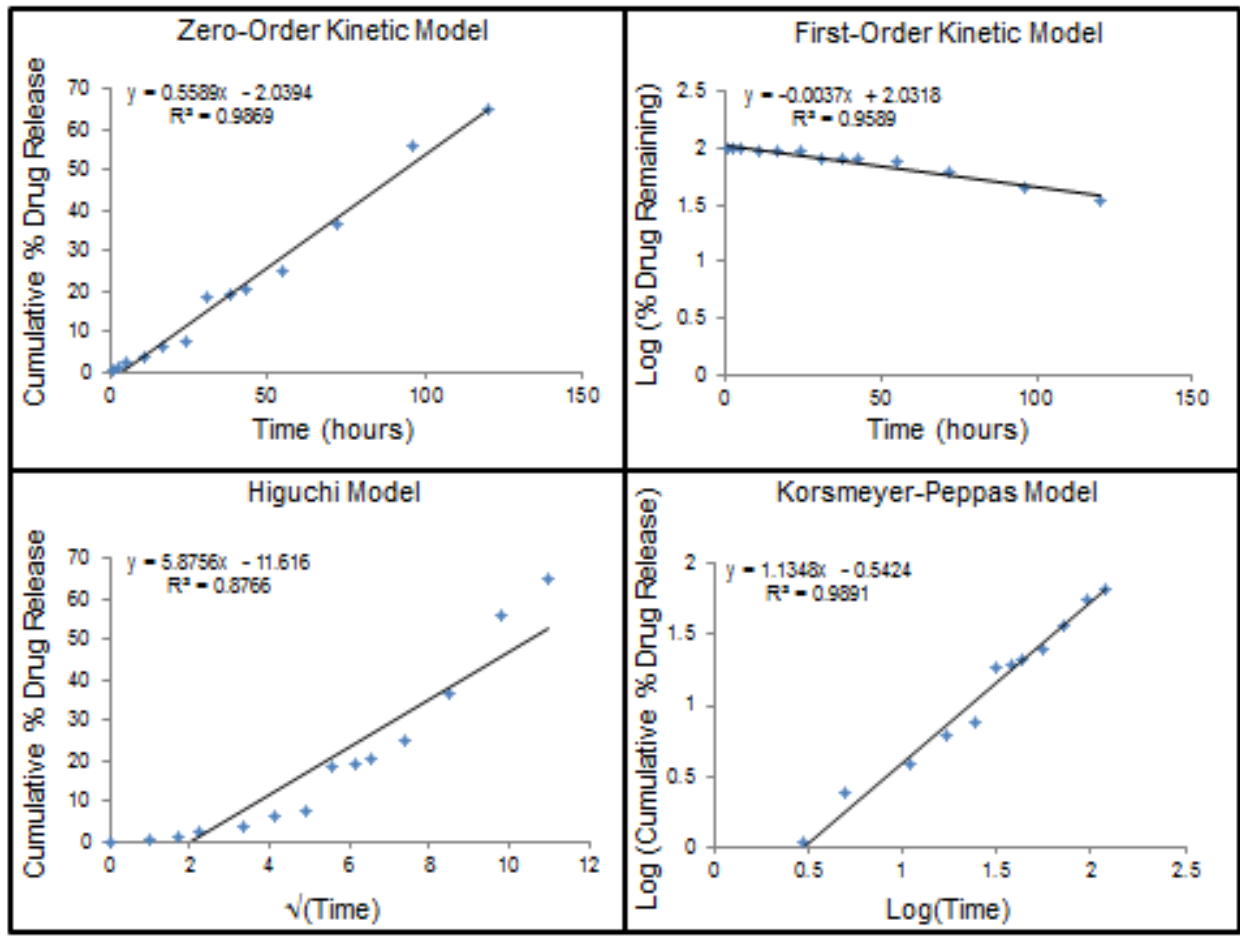

Figure 26. Paclitaxel release from three layer gold nanoparticles (points, representing average values) fitted to kinetic models (lines). The first $60 \%$ of cumulative release was fitted to each kinetic model: zero-order kinetic model by plotting cumulative \% drug release vs. time, first-order kinetic model by plotting log of $\%$ drug remaining vs. time, simplified Higuchi model by plotting cumulative $\%$ drug release vs. square root of time, and Korsmeyer-Peppas model by plotting log cumulative \% drug release vs. log time. Both the zeroorder kinetic and the Korsmeyer-Peppas models showed high correlation with $\mathrm{R}^{2}>0.98$. 


\section{Discussion}

We synthesized two and three layer gold nanoparticles to analyze the effect of surface modifications on the loading and release kinetics of two commonly utilized chemotherapeutics, cisplatin and paclitaxel, representing hydrophilic and hydrophobic drugs, respectively. Two layer gold nanoparticles were synthesized through the addition of a TL layer and PC coating [225], thus creating a hydrophobic region accessible to water insoluble drugs such as paclitaxel (Figure 20). Besides aiding in drug entrapment, a PC coating around gold nanoparticles was previously shown to significantly reduce nanoparticle cytotoxicity [357]. PC-coated gold nanoparticles were synthesized by first displacing the citrate stabilizer with TL. The strong binding affinity felt by the head group of TL for the gold core creates water insoluble nanoparticles, as the hydrophobic tails of TL point outward from the gold cores (Figure 20). Addition of PC to the outer layer of TL re-establishes water solubility of the nanoparticles, as the tail of the PC molecule binds tail-to-tail with TL. This process is expected to effectively create a hydrophobic region between the TL and PC layers that can be utilized for loading hydrophobic drugs. The two layer nanoparticles may be considered analogous to liposomes, yet containing an inner gold core. Addition of the bilayer to the outside of gold nanoparticles is expected to increase the bioavailability and decrease immunogenicity, as PC is a primary component of all cellular membranes.

Three layer gold nanoparticles were synthesized through the addition of HDL to the surface of PCcoated two layer gold nanoparticles. This modification is expected to enhance tumor-targeting capabilities, especially for hepatocellular carcinoma as HDL receptors are unregulated in liver cancer [329]. Cisplatin was loaded after the addition of PC for the two layer gold nanoparticles or after the addition of HDL for the three layer gold nanoparticles, and expected to exhibit faster release kinetics in comparison to paclitaxel due to the weakness of the non-covalent linkages (Figure 20).

Nanoparticles were characterized to confirm proper synthesis and modifications. Currently, a set of characterization standards for characterizing nanoparticles does not exist [399], thus this study utilized common instrumentation to ensure size, surface charge, and surface functionalization. While SEM (Figure 21) and DLS (Figure 22) are two common techniques for determining nanoparticle size [400], measurement variances are often seen between samples using both instruments [401]. Hydrodynamic sizes of nanoparticles obtained via DLS are often larger than sizes determined through SEM analysis, yet this is 
common as DLS considers the effects of Brownian motion in solution [401]. Nanoparticle surface charge can affect the transport of nanoparticles in vivo. As the head groups of PC are negatively charged, it was expected that two layer nanoparticles would be moderately anionic. A previous study showed that the zeta potential of PC-coated nanoparticles varies based upon $\mathrm{pH}$, from $14 \mathrm{mV}$ at $\mathrm{pH}=5$ to -40 at $\mathrm{pH}=7$ [402]. Due to the orientation of the PC onto the nanoparticles on top of the thiol compound, a slightly negative zeta potential was obtained. The zeta potential of HDL coated nanoparticles was expected to be more neutrally charged as HDL is a neutrally charged molecule [403]. These expectations were confirmed, as shown in Table 1.

Previous studies have shown that highly cationic or anionic nanoparticles experience increased uptake in the liver, thus inactivating the nanoparticles before they have time to reach the target destination and resulting in possible liver toxicity [404-406]. It has been shown that nanoparticles with a slightly negative charge may have low liver uptake and enhanced accumulation in solid tumors [404], thus suggesting that nanoparticles with slightly negative surface charges will display improved biocompatibility, reduced RES sequestering, and enhanced drug delivery to solid tumors.

FTIR confirmed the presence of surface modifications by comparing the peaks of two and three layer gold nanoparticles to pure PC and HDL (Figure 23). Two layer gold nanoparticles coated with TL and PC were expected to have a large peak associated with $-\mathrm{CH}_{2}$ and $-\mathrm{CH}_{3}$ groups $\left(\sim 3000 \mathrm{~cm}^{-1}\right)$ along with phosphate group vibrations $\sim 900 \mathrm{~cm}^{-1}[334,407]$. While these bands were present in the nanoparticle spectra, the intensity of the peaks were diminished from the spectra of pure PC. This can be attributed to the layering process, as the PC is loaded on top of TL, and both are attached to gold cores. For HDL-coated nanoparticles, bands were expected to show with lipid esters between $1700-1800 \mathrm{~cm}^{-1}$ and two amide stretches between $1500-1700 \mathrm{~cm}^{-1}$ [333]. As the HDL is loaded on top of the PC-coated nanoparticles, PC representative peaks were expected to be visible in the spectra of the three layer gold nanoparticles.

Cisplatin release from two and three layer gold nanoparticles showed an initial burst during the first five hours followed by a steady release for the following 14 days (Figure 24 A-B). An initial burst is common for nanoparticles, yet is highly dependent upon surface polymers and strength of drug attachment [408]. As cisplatin was bound to the nanoparticles non-covalently, the initial burst was expected. In comparison, paclitaxel showed a steady drug release profile (Figure $24 \mathrm{C}-\mathrm{D}$ ). Minimal paclitaxel was 
released within 14 days from the two-layer formulation, which can be attributed to its tight encapsulation within the hydrophobic layer created by the TL and PC. While PC may be degraded inside the body, it will stay relatively intact in PBS, thus not allowing most of the drug to escape.

However, the addition of HDL to the surface of PC disrupts the layer allowing for paclitaxel to slowly release from the hydrophobic region. This hypothesis is supported by the work of Scherphof et al. who determined that HDL could disrupt the structural integrity of liposomes synthesized with PC [409]. This previous work demonstrated that albumin encapsulated in liposomes could be effectively released by adding HDL to the solution [409]. This effect could explain the difference between the two and three layer gold nanoparticles loaded with paclitaxel, showing a 5-fold increase in release from the three layer formulation coated with HDL in comparison to the two layer (Figure 24 C-D). This also suggests that the addition of HDL may not be creating an actual layer on the outside of the nanoparticles, yet only inserting itself into the PC layer.

Paclitaxel release was best fitted by the simplified Higuchi model for the two layer nanoparticles and the Korsmeyer-Peppas model and zero-order kinetic model for the three layer nanoparticles, both with correlation values $>0.98$. For these nanoparticles, the long term sustained release could make them suitable candidates for therapeutic applications. Cisplatin release from two and three layer gold nanoparticles was best modeled by the Korsmeyer-Peppas equation. Both the simplified Higuchi model and KorsmeyerPeppas models describe drug release from degrading matrix and polymeric systems, thus suggesting that the aggregate drug released from multiple gold particles confined within a dialysis bag may be modeled similar to a system which undergoes degradation. The Higuchi model is based upon the following assumptions: (1) diffusion of drug only occurs in a single dimension, (2) negligible matrix swelling and dissolution, (3) much smaller drug molecules than system thickness, (4) constant drug diffusivity, (5) release environment acts as a perfect sink, and (6) much higher drug solubility than matrix initial drug concentration [32]. The Korsmeyer-Peppas model is a semi-empirical relation also known as the power law, in which the fraction of drug release is exponentially related to the time for release. Two main assumptions include: (1) the equation is only applicable for the first $60 \%$ of drug release and (2) the release must occur in a single dimension $[410,411]$. The single dimension is constructed by the release of drug radially outward from the source, thus making it possible to model a 1-D problem. 
For comparison, we also evaluated the Weibull model as a possible candidate for describing the drug release. While this model is a general empirical equation that is widely applied to drug release from pharmaceutical dosage forms, the model is limited by the inability to establish in vivo and in vitro correlation and the lack of parameters that can be related to the drug dissolution rate [410]. The Weibull model exhibited low correlation for cisplatin-loaded two and three layer gold nanoparticles, with $\mathrm{R}^{2}$ values of 0.7617 and 0.8792 , respectively. The model was a better fit for the paclitaxel-loaded nanoparticles, with the two layer gold nanoparticles having $\mathrm{R}^{2}=0.9313$ and the three layer formulation with $\mathrm{R}^{2}=0.9743$.

In contrast to polymeric or matrix nano-materials in which drugs are loaded within the nanocarrier structure, gold surfaces allow for drug molecule attachment via charge interactions and thiol-gold bonds that approach covalent bonds in strength. Based on desired release profiles and sequestration of molecules due to particular physical properties (such as charge and hydrophobicity), there may be applications for which a layered system is easier to design using gold instead of polymeric nano-materials. Thus, citrate gold particles represent an initial step to build a multilayer system on a gold surface, which can be used to elucidate interactions with cells. The next step would be to transition to a gold coated particle capable of absorbing light at a specified wavelength to create heat and to use this energy to release drugs from the nanoparticle, thus leading to enhanced localized delivery. However, to enhance release requires particles which absorb light in a region transparent to tissue, such as near-infrared absorbing gold nanoparticles (nanorods, gold silica nanoshells or gold-sulfide aggregate nanoparticles). Citrate gold colloid based particles are unsuitable for thermal absorption as the wavelength of light used to activate these particles $(\sim 540 \mathrm{~nm})$ will harm living tissues due to absorption of energy at this wavelength [412].

Enhanced understanding of hydrophilic and hydrophobic drug release kinetics from multi-layered gold nanoparticles can result in the development of combinatorial treatment strategies targeting tumor cells. This study analyzes the release kinetics of cisplatin and paclitaxel from TL, PC, and HDL coated versions of citrate gold nanoparticles as a first step in this evaluation. The results may further help to calibrate computational simulations that can provide insight into the complex dynamics of nanoparticle transport and drug release within solid tumors [336, 371, 413]. 


\title{
CHAPTER V
}

\section{CYTOTOXICITY OF 2- AND 3- LAYER GOLD NANOPARTICLES IN 3D MODELS OF LUNG CANCER}

\author{
$\underline{\text { Background }}$
}

\section{Chemotherapeutic Delivery to Solid Tumors}

Formation of hypoxic and necrotic portions of solid tumors arise from inefficient vascularization and irregular angiogenesis, as the lack of blood vessel organization results in hypo-vascularization and nutrient deprivation [414]. Similarly, chemotherapeutic delivery in vivo is hindered by diffusion limitations and the increased interstitial fluid pressure (IFP) of solid tumors [415]. While circulating drug can exit the highly fenestrated capillaries of the tumor [416], various biological barriers hinder the drug particle from diffusing beyond 3-5 cell diameters from the extravasation site [417, 418]. Treatment failure results if a therapeutic concentration of chemotherapeutic fails to effectively reach all cancer cells, including those in hypo-vascularized portions of tumor [415]. As tumors elicit a 3-dimsensional (3D) morphology in the body, it is important to understand the spatial geometry of drug diffusion within 3D cell cultures to develop novel treatment modalities [419]. Several groups have incorporated the use of 3D cell cultures in computational models to better understand the mechanisms of nutrient diffusion and drug transport in cancerous spheroids $[413,420]$.

\section{Cell Cultures: Monolayer versus Spheroid}

While 2-dimensional (2D) monolayer cell cultures are commonly utilized for cytotoxicity testing, they lack the physiological features of the in vivo condition and the spatial response of drug activity [421]. 3D cell cultures are a closer representation of the morphological environment of solid tumors found within the body, as they mimic the effects of the ECM components, nutrient diffusion gradients, and IFP alterations [422, 423]. Thus, 3D cell cultures can provide additional details concerning the effectiveness and toxicity of drugs not attainable through monolayer cell cultures. For example, Godugu et al. showed 
that the $\mathrm{IC}_{50}$ value of chemotherapeutics would increase between monolayer and $3 \mathrm{D}$ cell cultures, which was linked to higher drug resistance in the 3D model [424]. While 3D cell cultures require more care and planning then 2D cell cultures with a higher cost, newer methodologies such as hanging drop arrays described by Tung et al. make it possible to perform high-throughput screening of compounds in 3D cell cultures [425].

In 2014, lung cancer remains the leading cause of cancer-related death in the United States with an overall 5-year survival rate of $17 \%$ [56]. While treatment options exist, the late onset of symptoms results in $85 \%$ of cases being diagnosed at advanced disease when the 5-year survival rate is only $4 \%$ [56]. Current treatment options for patients with inoperable tumors include chemotherapeutics and radiation therapy, yet these options only provide an average extension of life between 2-12 months [426, 427]. Oftentimes, chemotherapeutic treatment results in severe off-target toxicity as the compounds distributes to all tissues in the body $[428,429]$, treatment failure as the drug molecules cannot reach all the cells residing within the tumor due to inefficient vascularization [430], or the development of resistance mechanisms by the cancerous cells such as P-glycoprotein by cancer cells can render the drug compound ineffective [431].

Research studies are underway to determine optimal methods for overcoming each of the issues in order to more effectively treatment solid tumors. Previous studies utilizing nanoparticles for lung cancer treatment have shown great potential for the treatment of lung cancer (please refer to the following review papers: $[432,433])$.

\section{Purpose of Study}

The last decade has shown that nanoparticles can be effective in the treatment of solid tumors for several reasons, including their passive targeting capabilities resulting from the EPR effect [433], improved ability to avoid systemic distribution by active targeting [434], and their avoidance of resistance mechanisms by undergoing distinct mechanisms of endocytosis [435]. Additionally, the large surface area to volume ratio of nanoparticles can be functionalization with polymers, drugs, or other compounds to enhance targeting capabilities and bioavailability [436]. Cisplatin and paclitaxel are two commonly utilized chemotherapeutics for the treatment of NSCLC [437], with cisplatin functioning in the nucleus by disturbing DNA replication and paclitaxel functioning by stabilizing microtubules in the cytoplasm [438, 439]. 
In this study, gold nanoparticles were modified with thiol and PC in the formation of a two-layer system or thiol, PC, and HDL for a three-layer system. We have previously shown that both two- and threelayer gold nanoparticles showed superior diffusivity in comparison to PEGylated nanoparticles in 3D cell cultures [225]. It was also shown that both two- and three layer gold nanoparticles could be loaded with cisplatin and or paclitaxel and showed unique drug unloading profiles [440]. This study examines the use of cisplatin or paclitaxel loaded nanoparticles in 2D and 3D cell cultures to determine their cytotoxicity in three NSCLC cell lines. 


\section{$\underline{\text { Methods }}$}

\section{Synthesis of Citrate Gold Nanoparticles}

Citrate gold nanoparticles were synthesized using a method in which gold chloroauric acid is reduced by trisodium citrate described by G. Frens in 1973 [306]. In this process, $2.2-2.4 \mathrm{~mL} \mathrm{1 \%}$ weight/volume (wt/v) trisodium citrate (Fisher Scientific, Watham, MA, USA) is added to $200 \mathrm{~mL}$ of boiling $0.01 \%$ wt/v HAuCl4 (Alfa Aesar, Ward Hill, MA, USA), and the solution is allowed to continue boiling for 10 minutes to promote the reaction of sodium citrate to citric acid. Once the reaction is completed, the solution cools at room temperature before concentrated using a rotovapor (Buchi Rotovapor System, BÜCHI Labortechnik AG, Flawil, Switzerland) to $20 \mathrm{~mL}$. The addition of surface modifications followed the protocol outlined below.

\section{Nanoparticle Functionalization}

1-Hexadecanethiol (Sigma Aldrich) was the first layer applied to the gold nanoparticles. The TL compound has a stronger binding affinity for the surface of the gold nanoparticles, thus displacing the citrate molecules. While stirring, $20 \mathrm{~mL}$ pure ethanol was placed in a beaker with $60 \mu \mathrm{L} 1$-Hexadecanethiol dissolved in ethanol added secondly. The 1-Hexadecanethiol solution was added slowly to the nanoparticle solution over the next 10 minutes, while also agitating the sample by sonication. After addition of 1Hexadecanethiol, the sample was agitated for two hours, and then placed for 12 hours on an orbital rocker (Boekel Scientific, Feasterville, PA, USA). The sample was spun down, and the pellet was washed twice and resuspended in chloroform. PC (Sigma Aldrich) was the second functionalization added to the nanoparticles. PC was solubilized in chloroform (Sigma Aldrich) and $100 \mu \mathrm{L}$ were added to the particles after the TL layer and allowed to set overnight on an orbital rocker. The solutions were transferred to glass tubes and the chloroform evaporated at ambient temperature. This process completed the two layer citrate gold nanoparticles containing gold core, TL, and PC. The three-layered nanoparticles were created by optimizing the ratio of HDL (Lee Biosolutions, St. Louis MO, USA) to particle optical density (1 mg HDL per 20 OD nanoparticle), and allowed to react overnight after two hours of agitation.

\section{Loading of Chemotherapeutic onto Nanoparticles}

Paclitaxel (Cayman Chemicals, Ann Arbor, MI, USA) and cisplatin (Sigma Aldrich) were loaded onto the nanoparticles in distinct regions to ensure optimal loading efficiency. Paclitaxel was loaded after 
the addition of 1-Hexadecathiol and before the addition of PC. Briefly, after nanoparticles were placed on an orbital shaker for 12 hours, the solution was removed. After nanoparticles were resuspended in $9 \mathrm{~mL}$ chloroform, an addition $1 \mathrm{~mL}$ of chloroform containing $5 \mathrm{mg}$ paclitaxel was added to the solution.

Nanoparticles were agitated for two hours using sonication before the solution was placed on an orbital rocker for six hours. The solution was further modified to add the second layer of PC to the surface of the nanoparticles. While paclitaxel was loaded into the hydrophobic region created between the TL and PC layer, cisplatin was loaded at two different areas dependent upon the layering.

For the two layer citrate gold nanoparticles, cisplatin was added after the addition of PC. This was done by transferring the solutions to glass tubes and the chloroform evaporated at ambient temperature. Next, the nanoparticles were resuspended in $10 \mathrm{~mL}$ ultrapure H2O (Purelab Ultra, Elga Labwater, UK) containing $7.5 \mathrm{mg}$ cisplatin. For the three layer citrate gold nanoparticles, cisplatin was added after the addition of HDL by synthesizing the particles as described above, yet after HDL was added to the particles and allowed to react for two hours, the solution was removed, and $7.5 \mathrm{mg}$ cisplatin was added. Excess chemotherapeutic was removed from the solution by centrifuging the particles at 7000 rpm for 25 minutes, removing the supernatant, and re-suspending the particles in the corresponding solvent. Washing was performed twice.

\section{Nanoparticle Characterization}

Nanoparticle identity was verified as follows: (1) Maximum absorption wavelengths were obtained using the Varian Cary 50 Bio Ultraviolet-Visible (UV-Vis) Spectrometer (McKinley Scientific); (2) size and zeta potential measurements were obtained using the ZetaSizer Nanoseries ZS90 (Malvern Instruments, Worcestshire, UK); (3) DLS (dynamic light scattering, also known as Photon Correlation Spectroscopy) to determine hydrodynamic size in solution based upon Brownian motion; (4) shape and size

were determined using a Zeiss Supra 35VP (Carl Zeiss, Oberkochen, Germany) SEM; (5) presence of lipids on the particle cores was confirmed using a FTIR (Perkin Elmer Spectrum BX; Perkin Elmer, Waltham, MA, USA) and through visual analysis using the SEM.

\section{Evaluation of In Vitro Drug Release}

The drug-loaded nanoparticles were placed into dialysis tubes and submerged into beakers containing $500 \mathrm{~mL} 1 \mathrm{X}$ PBS at $\mathrm{pH}$ 7.4. The dialysis tubing cellulose membrane had an average flat width of 
$25 \mathrm{~mm}$ and 12,000 MW cutoff (Fischer Scientific, Waltham, MA, USA) and was clipped at both ends to ensure no particles could exit. The beaker was continually agitated using a magnetic stirrer and covered with Parafilm to ensure no evaporation of PBS would take place as experiments were performed at $37^{\circ} \mathrm{C}$. At various time intervals, $3 \mathrm{~mL}$ samples of the PBS solution with drug were removed and replaced with fresh PBS to ensure a constant volume. Drug concentration of each sample was analyzed using HPLC. Cumulative drug release was found using the following equation (Eq. 8):

Cumulative Drug Release $(\%)=\frac{[\text { Drug }]_{t}}{[\text { Drug }]_{\text {total }}} \times 100$

where [Drug $]_{t}$ is the concentration of drug in the sample at time $t$ and [Drug $]_{\text {total }}$ is the total amount of drug loaded onto the nanoparticles.

\section{Cell Culture}

Three human NSCLC cell lines were used in this study: A-549, PC-9, NCI-H358. Cell lines were maintained in RPMI 1640 medium (Cellgro, Corning Inc.) supplemented with 10\% fetal bovine serum (Cellgro, Corning Inc.) and 1\% penicillin-streptomycin-glutamine solution (Cellgro, Corning Inc.) in a humidified atmosphere of $5 \% \mathrm{CO}_{2}$ at $37^{\circ} \mathrm{C}$. All cells were grown to $80 \%$ confluence before use in $2 \mathrm{D}$ or $3 \mathrm{D}$ cell cultures.

\section{Evaluation of Free Drug Cytotoxicity in 2D Cell Cultures}

A-549, PC-9, and NCI-H358 cells were seeded into 24 -well plates at a density of $2 \times 10^{4}$ cells per well, and incubated at $37^{\circ} \mathrm{C}$ for 24 hours. Media was removed from wells and replaced with $1 \mathrm{~mL}$ fresh RPMI-1640 media containing varying concentrations of drug for 48 hours. For cisplatin experiments, cells were exposed to the following concentrations for 48 hours: 1024, 256, 64, 16, 4, 1, 0.25, $0.0625 \mu \mathrm{M}$. For paclitaxel experiments, cells were exposed to the following concentrations for 48 hours: 1024, 256, 64, 16, 4, 1, 0.25, $0.0625 \mathrm{nM}$. After incubation, media was removed and cells were washed with 1X PBS. Cells were detached using $250 \mu \mathrm{L} 0.05 \%$ trypsin per well and an additional $250 \mu \mathrm{L}$ of fresh RPMI-1640 media was added to each well. Cells were counted visually using trypan blue (Cellgro, Corning Inc.) exclusion. For determining statistically significant differences, each case was done in quadruplicate $(n=4)$.

\section{Evaluation of Free Drug Cytotoxicity in 3D Cell Culture}

A-549, PC-9, and NCI-H358 cells were seeded into 24-well ultra-low cluster plates (Costar, Corning Inc.) at a density of $1 \times 10^{5}$ cells per well, and shaken for $\sim 10$ minutes to promote aggregation of 
cells. Cells were then placed in an incubator $37^{\circ} \mathrm{C}$ for 5 days for spheroid acclimation. After spheroid formation, the media was removed while carefully avoiding the spheroid. $1 \mathrm{~mL}$ of media containing either cisplatin or paclitaxel at varying concentrations was added to the corresponding wells. For cisplatin experiments, cells were exposed to the following concentrations: $1024,256,64,16,4,1,0.25,0.0625 \mu \mathrm{M}$. For paclitaxel experiments, cells were exposed to the following concentrations: 1024, 256, 64, 16, 4, 1, $0.25,0.0625 \mathrm{nM}$. Spheroids were treated with drugs were incubated at $37^{\circ} \mathrm{C}$ for 48 hours. After 48 hours, drug-containing media was removed. $500 \mu \mathrm{L} 0.05 \%$ trypsin was added to detach the individual cells of the spheroid from each other and $500 \mu \mathrm{L}$ fresh RPMI-1640 media was added to deactivate the trypsin. Cells were counted visually using trypan blue exclusion. For statistically significant differences, each case was done in quadruplicate $(\mathrm{n}=4)$.

\section{Evaluation of Drug Loaded Nanoparticles in 3D Cell Culture}

A-549, PC-9, and NCI-H358 cells were seeded into 24-well ultra-low cluster plates at a density of $1 \times 10^{5}$ cells per well, and shaken for $\sim 10$ minutes to promote aggregation of cells. Cells were then placed in an incubator $37^{\circ} \mathrm{C}$ for 5 days. Spheroids were exposed to the same concentration of nanoparticle-loaded drug as free drug (see above). The dose of drug-loaded nanoparticle was calculated by considering two parameters: (1) the loading efficiency from HPLC data showed the exact concentration of drug on nanoparticles and (2) the percent of drug released at 48 hours. By considering both of these factors, the amount of drug needed to reach each concentration was calculated. 


\section{$\underline{\text { Results }}$}

\section{Characterization of Nanoparticles}

Diffusivity of two and three layer gold nanoparticles was previously shown to be superior in comparison to PEGylated nanoparticles when tested in 3D cell cultures [225]. When these nanoparticles were loaded with cisplatin or paclitaxel, they displayed unique release curves with cisplatin having an initial burst of drug release followed by a steady release and paclitaxel displaying a sustained release curve over a period of 14 days [440]. Here, we examine the cytotoxicity of two and three layer drug-loaded nanoparticles in 2D and 3D cell cultures (Figure 27). Two layer nanoparticles were synthesized by first adding TL and secondly PC, which created a hydrophobic region capable for loading water-insoluble compounds. The three-layer nanoparticle contained an additional modification of the outer PC layer of HDL.

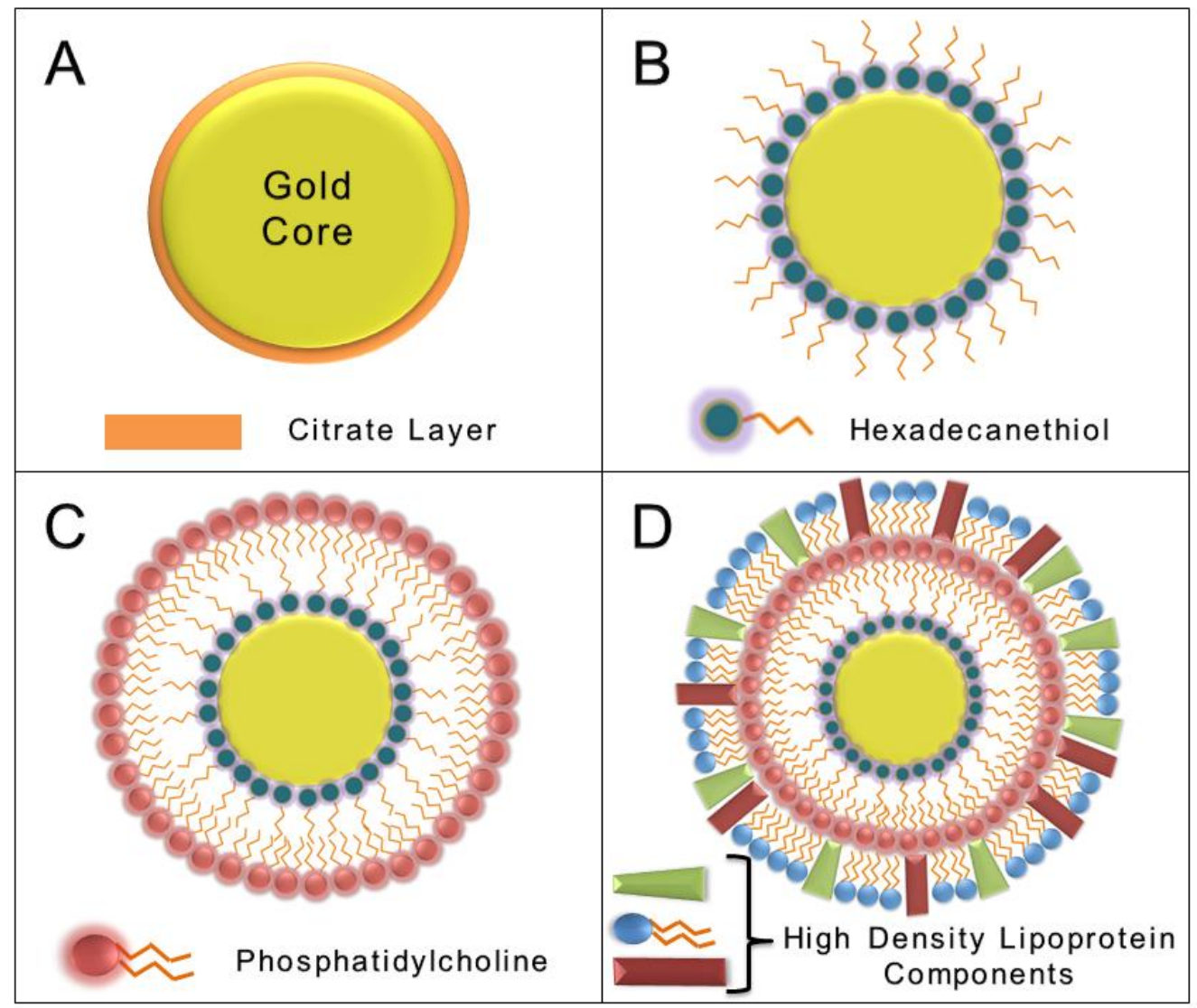

Figure 27. Synthesis of two- and three layered gold nanoparticles. Nanoparticles were functionalized by a layering process as depicted in this figure. (A) Before functionalization, gold nanoparticles were 
synthesized using a sodium citrate method resulting in a citrate-covered nanoparticle. (B) Hexadecanethiol was added to the nanoparticle solution that displaced the citrate molecules, forming water-insoluble nanoparticles. (C) To create a region suitable for loading of hydrophobic drugs, PC was added to the nanoparticle solution, binding tail-to-tail with the thiol layer. (D) To further functionalize the nanoparticles, a three-layer system was created by adding HDL to the surface of the PC-coated system.

Confirmation of the particle identity was gained through characterized using several techniques, including: ultraviolet-visible (UV-Vis) spectroscopy to determine the maximum absorbance values, dynamic light scattering (DLS) to determine hydrodynamic size in solution based upon Brownian motion, SEM for analyzing the shape and size of particles, zeta potential for studying surface charge alterations, and FTIR spectroscopy to determine surface modifications.

UV-Vis spectroscopy was performed to ensure nanoparticles were not agglomerating in solution (add citation). Two and three layer nanoparticles displayed similar spectra with a shift of $\sim 4 \mathrm{~nm}$, with the two layer nanoparticles having a maximum absorbance peak of $539 \mathrm{~nm}$ and the three layer nanoparticles displaying a peak at $535 \mathrm{~nm}$ (Figure 28). Using SEM, sizing of two and three layer gold nanoparticles was shown to be $54.7 \pm 7.9 \mathrm{~nm}$ and $62.1 \pm 8.8 \mathrm{~nm}$, respectively (Figure 29). DLS provided further details regarding the sizing of nanoparticles when in solution based upon the effects of Brownian motion. Sizing from DLS is based upon the thin electric dipole layer of solvent that adheres to the surface of nanoparticles [441]. The hydrodynamic size for two and three layer gold nanoparticles was $71.5 \pm 9.7 \mathrm{~nm}$ and $80.2 \pm 12.4$ nm, respectively (Table 5). Further characterization utilizing zeta potential analysis showed that three layer nanoparticles displayed a relatively neutral surface charge of $-6 \mathrm{mV}$, while two layer nanoparticles were more anionic at $-21 \mathrm{mV}$ (Table 5). 

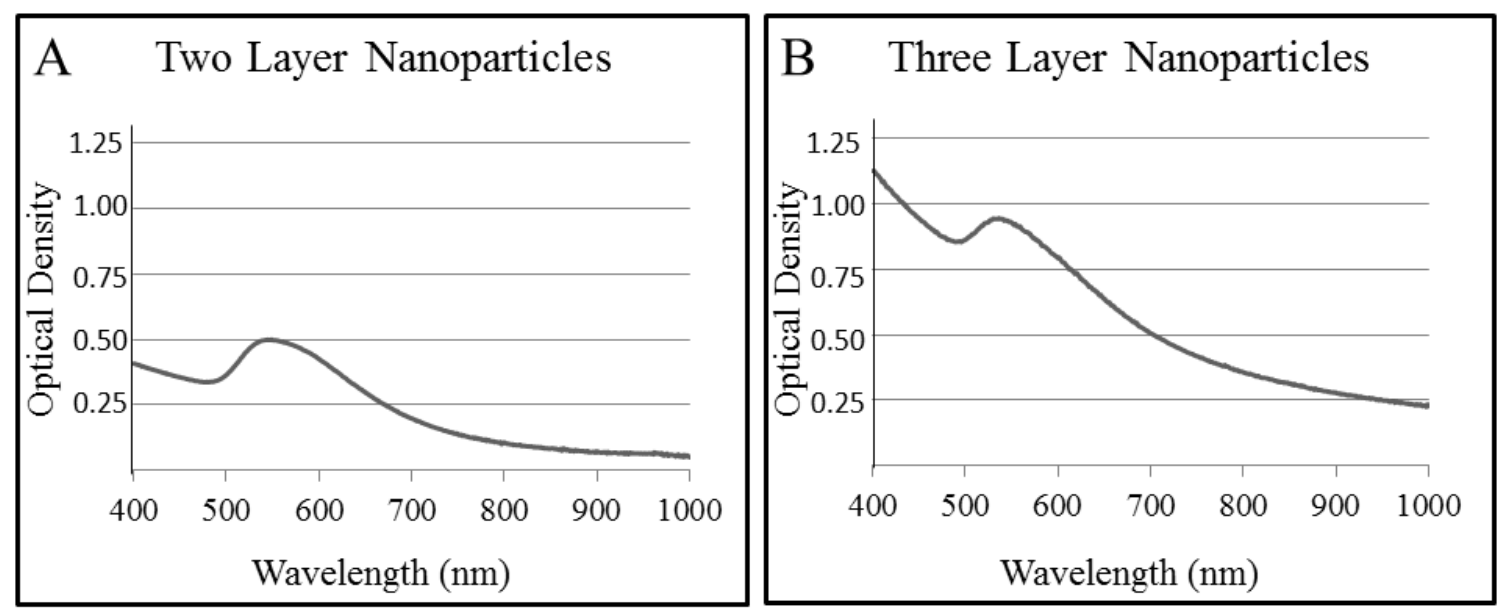

Figure 28. Gold nanoparticles were characterized using UV-Vis spectroscopy to determine the maximum absorbance wavelength. The maximum wavelength for the two layer gold nanoparticles was $539 \mathrm{~nm}$, while the three layer gold nanoparticles produced a maximum absorbance of $535 \mathrm{~nm}$.

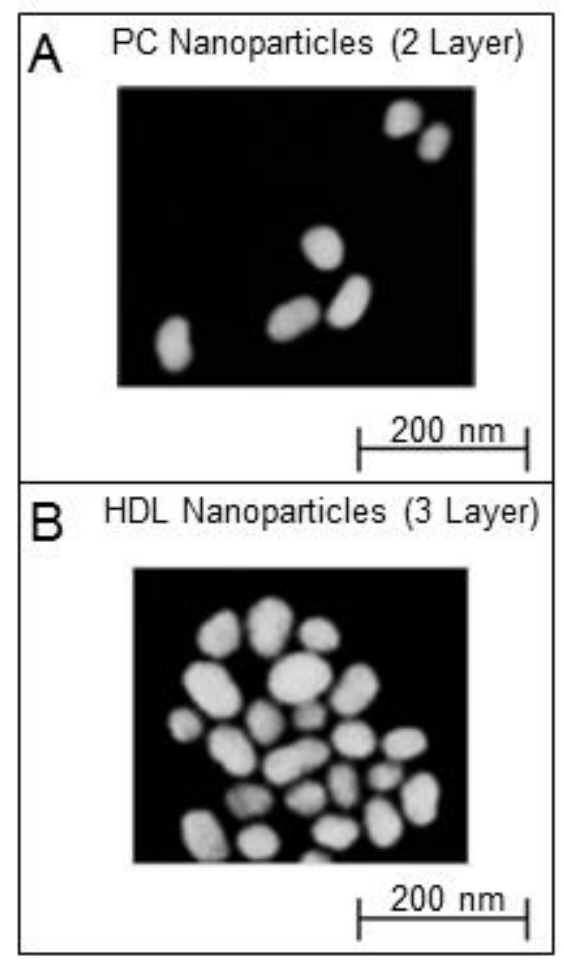

Figure 29. Scanning electron microscopy was utilized to determine the size and shape of two and three layer nanoparticles. Gold nanoparticles were mostly spherical in shape, yet contained a heterogeneous 
assortment of nanoparticle sizes. On average, the two and three layer nanoparticles were determined to be $54.7 \pm 7.9 \mathrm{~nm}$ and $62.1 \pm 8.8 \mathrm{~nm}$, respectively.

\section{Table 5}

Characterization of 2- and 3-Layer Nanoparticles using UV-Visible Spectroscopy, Dynamic Light Scattering, and Zeta Potential Analysis.

\begin{tabular}{|l|c|c|c|}
\hline & $\begin{array}{c}\text { Max Wavelength } \\
\text { (wavenumber-1) }\end{array}$ & DLS (nm) & $\begin{array}{c}\text { Zeta Potential } \\
(\mathbf{m V})\end{array}$ \\
\hline 2-Layer Nanoparticles (TL/PC) & 539 & $71.5(9.7)$ & $-21 \mathrm{mV}$ \\
\hline 3-Layer Nanoparticles (TL/PC/HDL) & 535 & $80.2(12.4)$ & $-6 \mathrm{mV}$ \\
\hline
\end{tabular}

FTIR was utilized to confirm the presence of PC and HDL onto the surface of nanoparticles (Figure 30). The spectra of pure PC and HDL were used for comparison [333, 397]. There were several peaks used to confirm the presence of TL and PC on the gold nanoparticles. Some of the key peaks included a $\left[\left(-\mathrm{CH}_{2}\right)_{\mathrm{n}}\right]$ rocking vibration at $720 \mathrm{~cm}^{-1}, \mathrm{PO}_{4}{ }^{3-}$ group vibrations at $900 \mathrm{~cm}^{-1}$, a $\mathrm{C}-\mathrm{O}-\mathrm{C}$ stretch at $1100 \mathrm{~cm}^{-1}$, and an asymmetric and symmetric $-\mathrm{CH}_{2}$ at $2880 \mathrm{~cm}^{-1}$ and $-\mathrm{CH}_{3}$ at $2950 \mathrm{~cm}^{-1}$ stretch and vibration. Additional peaks were associated with the other chemicals used to synthesize the particles. HDLcoated nanoparticles exhibited several similar peaks to PC-coated nanoparticles, which were expected due to the layering process. The asymmetric and symmetric $-\mathrm{CH}_{2}\left(2880 \mathrm{~cm}^{-1}\right)$ and $-\mathrm{CH}_{3}\left(2950 \mathrm{~cm}^{-1}\right)$ stretch and vibration were still present from the PC-coating, yet several signature peaks of HDL also became visible. These peaks included a $\mathrm{C}=\mathrm{O}$ from the lipid ester between $1700-1800 \mathrm{~cm}^{-1}$, an amide bond stretches between $1500-1700 \mathrm{~cm}^{-1}$, and a phospholipid $\mathrm{P}=\mathrm{O}_{2}$ stretch at $1250 \mathrm{~cm}^{-1}$. 


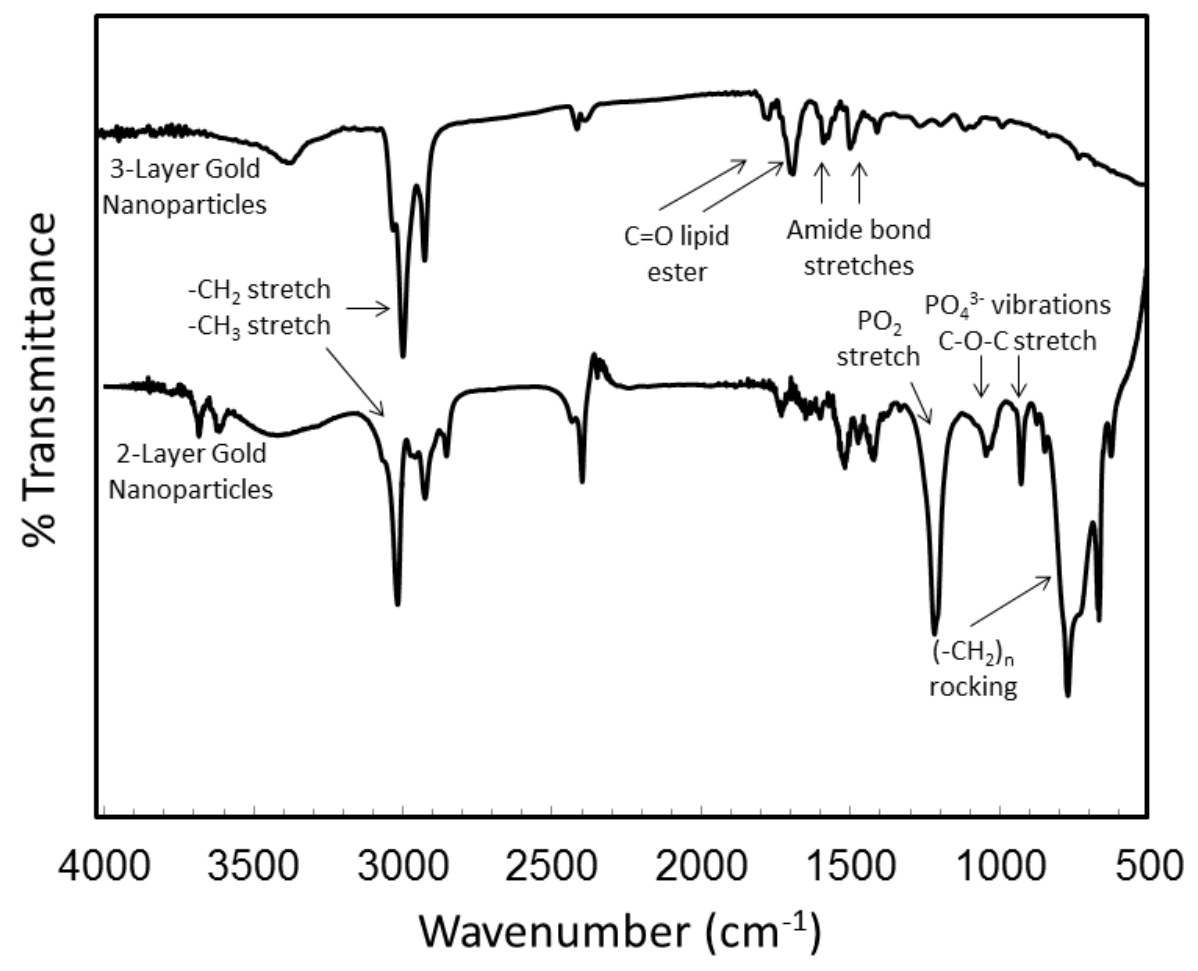

Figure 30. FTIR spectroscopy of Nanoparticles. Spectra of pure PC and HDL were used for comparison. Peaks used to confirm the presence of PC included a $\left[\left(-\mathrm{CH}_{2}\right)_{\mathrm{n}}\right]$ rocking vibration at $720 \mathrm{~cm}^{-1}, \mathrm{PO}_{4}{ }^{3-}$ group vibrations at $900 \mathrm{~cm}^{-1}$, a C-O-C stretch at $1100 \mathrm{~cm}^{-1}$, and an asymmetric and symmetric $-\mathrm{CH}_{2}$ at 2880 $\mathrm{cm}^{-1}$ and $-\mathrm{CH}_{3}$ at $2950 \mathrm{~cm}^{-1}$ stretch and vibration. HDL-nanoparticles exhibited additional peaks, including a $\mathrm{C}=\mathrm{O}$ from the lipid ester between $1700-1800 \mathrm{~cm}^{-1}$, an amide bond stretches between 1500 $1700 \mathrm{~cm}^{-1}$, and a phospholipid $\mathrm{P}=\mathrm{O}_{2}$ stretch at $1250 \mathrm{~cm}^{-1}$.

\section{Drug Release Kinetics}

Release of paclitaxel and cisplatin from both two- and three layer nanoparticles was dependent upon the type of drug loaded and the number of layers on the nanoparticle core. Paclitaxel was shown to release drug much slower than cisplatin, which experienced an initial burst of drug release (Table 6). The cumulative percent of paclitaxel released from the nanoparticles during the first 3 hours was $1.42 \pm 0.12 \%$ and $2.39 \pm 0.27 \%$ for the two- and three layer nanoparticles, respectively. For the cisplatin-loaded system, the two layer nanoparticles released $33.8 \pm 2.6 \%$ of drug within the first 3 hours, while the three layer system released $\sim 2 \mathrm{X}$ this amount $(59.1 \pm 2.0 \%)$. At the end of 96 hours, none of the nanoparticle systems had released the total amount of drug loaded. Results from a longer release experiment of 14 days have 
been published [440]. Three layer nanoparticles loaded with cisplatin released the most amount of drug $(78.9 \pm 2.1 \%)$ at the end of 96 hours as compared to the two-layer system $(49.7 \pm 0.70 \%)$. For the paclitaxel-loaded nanoparticles, the three layer system released $5 \mathrm{X}$ more drug as compared to the two layer system.

\section{Table 6}

Cumulative Release of Drug at Various Time Intervals with Associated Loading Efficiency [440]

\begin{tabular}{|l|c|c|c|c|c|c|c|}
\hline & $\begin{array}{c}\mathbf{3} \\
\text { Hours }\end{array}$ & $\begin{array}{c}\mathbf{1 2} \\
\text { Hours }\end{array}$ & $\begin{array}{c}\mathbf{2 4} \\
\text { Hours }\end{array}$ & $\begin{array}{c}\mathbf{4 8} \\
\text { Hours }\end{array}$ & $\begin{array}{c}\mathbf{7 2} \\
\text { Hours }\end{array}$ & $\begin{array}{c}\mathbf{9 6} \\
\text { Hours }\end{array}$ & $\begin{array}{c}\text { Loading } \\
\text { Efficiency }\end{array}$ \\
\hline 2-Layer & 33.8 & 37.6 & 43.0 & 46.9 & 48.9 & 49.7 & $68.4(7.1)$ \\
Nanoparticles & $(2.6)$ & $(2.7)$ & $(1.1)$ & $(1.5)$ & $(0.51)$ & $(0.70)$ & \\
w/Cisplatin & & & & & & & \\
\hline 2-Layer & 1.42 & 3.38 & 3.80 & 8.20 & 10.8 & 11.9 & $99.1(0.7)$ \\
$\begin{array}{l}\text { Nanoparticles } \\
\text { w/Paclitaxel }\end{array}$ & $(0.12)$ & $(0.13)$ & $(0.11)$ & $(0.09)$ & $(0.11)$ & $(0.90)$ & \\
\hline 3-Layer & 59.1 & 70.2 & 72.8 & 76.7 & 77.7 & 78.9 & $78.9(0.7)$ \\
$\begin{array}{l}\text { Nanoparticles } \\
\text { w/Cisplatin }\end{array}$ & $(2.0)$ & $(0.94)$ & $(0.87)$ & $(1.84)$ & $(1.1)$ & $(2.1)$ & \\
\hline 3-Layer & 2.39 & 3.88 & 7.50 & 23.1 & 36.5 & 55.7 & $99.4(0.4)$ \\
$\begin{array}{l}\text { Nanoparticles } \\
\text { w/Paclitaxel }\end{array}$ & $(0.27)$ & $(0.10)$ & $(0.9)$ & $(4.2)$ & $(4.3)$ & $(4.7)$ & \\
\hline
\end{tabular}

Since cytotoxicity measurements were performed at 48 hours for cell culture experiments, the amount of drug release at 48 hours from each of the nanoparticle systems was noted (Table 6). The two layer nanoparticles released $46.9 \pm 1.5 \%$ of cisplatin and $8.20 \pm 0.09 \%$ of paclitaxel at 48 hours in PBS. The three layer nanoparticles released $76.7 \pm 1.84 \%$ of cisplatin and $36.5 \pm 4.3 \%$ of paclitaxel at 48 hours. The loading efficiency was determined by subtracting the amount of unbound drug from the amount of drug utilized to synthesize the nanoparticles. The loading efficiency of two layer nanoparticles was $68.4 \pm$ $7.1 \%$ of cisplatin and $99.1 \pm 0.7 \%$ of paclitaxel. For the three layer nanoparticles, the loading efficiency of cisplatin was $78.9 \pm 4.9 \%$ and paclitaxel was $99.4 \pm 0.4 \%$.

\section{Cytotoxicity of Free Drug in $2 D$ and $3 D$ Cell Cultures}

The cytotoxicity of both free cisplatin and paclitaxel were measured in 2D and 3D cell cultures at 48 hours, as depicted in Figure 31. It was expected that 2D cell cultures would experience higher levels of cytotoxicity as lower concentrations of drug in comparison to 3D cell cultures, as monolayer cell cultures lack 3D morphology. In each cell line, 3D cell cultures showed more cell viability at higher concentrations 
of drug (Figure 31 A, C, E). Similar results were obtained for paclitaxel cytotoxicity experiments in A549, H358, and PC9 cell lines (Figure 31 B, D, F). While lower concentrations did not correlate with significant cytotoxicity differences, higher concentrations of paclitaxel produced a significant difference between the cytotoxic effects in 2D versus 3D cell cultures. In each case, cells residing within 3D cell culture provided to be more difficult to induce cytotoxicity in comparison to cells of monolayer cultures.

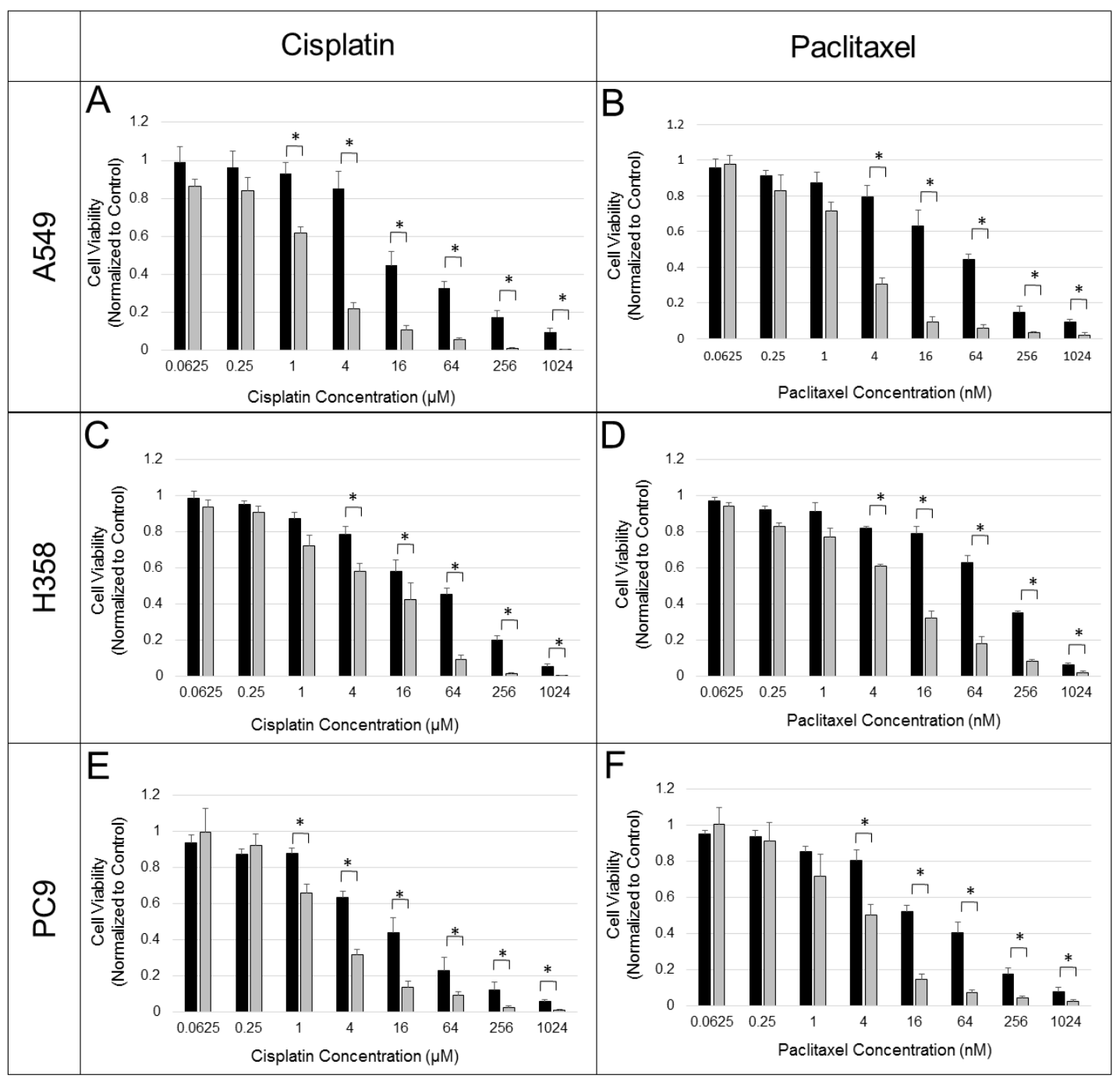

Figure 31. Cytotoxicity of free cisplatin and paclitaxel in three NSCLC (A549, H358, and PC9) cancer cell lines. For reference, black bars represent free drug in 3D cell cultures, while grey bars are indicative of free drug in $2 \mathrm{D}$ (i.e. monolayer) cell cultures. In all cases, the concentration of drug required to cause cytotoxicity in 3D cells was higher than concentrations needed in 2D cell cultures. At concentrations of 1 
$\mu \mathrm{M}$ or higher for cisplatin (A,C,E), the difference in cell viability between 3D and 2D cell cultures was significant. Concentrations of paclitaxel above $4 \mathrm{nM}$ showed similar results (B,D,F).

\section{Cytotoxicity of Drug-Loaded Nanoparticles in 3D Cell Cultures}

Cytotoxicity of drug-loaded nanoparticles was tested in 3D cell cultures to determine their effectiveness as potential therapies. For this reason, drug-loaded nanoparticles were compared to free drug. Cisplatin-loaded two and three layer nanoparticles were tested in 3D cell cultures of A549, H358, and PC9 NSCLC cell lines. Similarly, paclitaxel-loaded nanoparticles were tested in the same cell lines with results shown in Figure 32. The cisplatin-loaded nanoparticles showed similar performance to free drug in 3D cell cultures at all concentrations. In A549 cell line, the two-layer platform outperformed the free drug at higher concentrations (65 and $256 \mu \mathrm{M}$ ) (Figure 32A). Both two- and three layer nanoparticles showed superior efficacy in comparison to free drug at higher concentrations $(16,64$, and $256 \mu \mathrm{M})$ in $\mathrm{H} 358$ 3D cell cultures (Figure 32B). The PC9 cell line showed so statistically significant differences between the cytotoxicity of free drug versus nanoparticle-loaded drug, yet the nanoparticle systems performed equally as well as free drug (Figure 32C), which would still have benefits for possible therapeutic uses.

Two and three layer nanoparticles loaded with paclitaxel were also tested in 3D cell cultures to determine their cytotoxicity in comparison to free paclitaxel (Figure 32). For the A549 (Figure 32B) and PC9 (Figure 32F) cell lines, no statistical differences were seen between the cytotoxicity caused by free paclitaxel versus the drug-loading nanoparticles. The cytotoxicity graphs do suggest that the nanoparticle platforms performed similarly to free paclitaxel in each case. For the H358 cell line, the two-layer nanoparticle outperformed free drug at higher concentrations of 65 and $256 \mathrm{nM}$ (Figure 32D). Similarly, the three-layer nanoparticle displayed similar cytotoxicity as the two layer nanoparticles at $256 \mathrm{nM}$.

Overall, paclitaxel-loaded nanoparticles performed similarly to free paclitaxel in each case. 


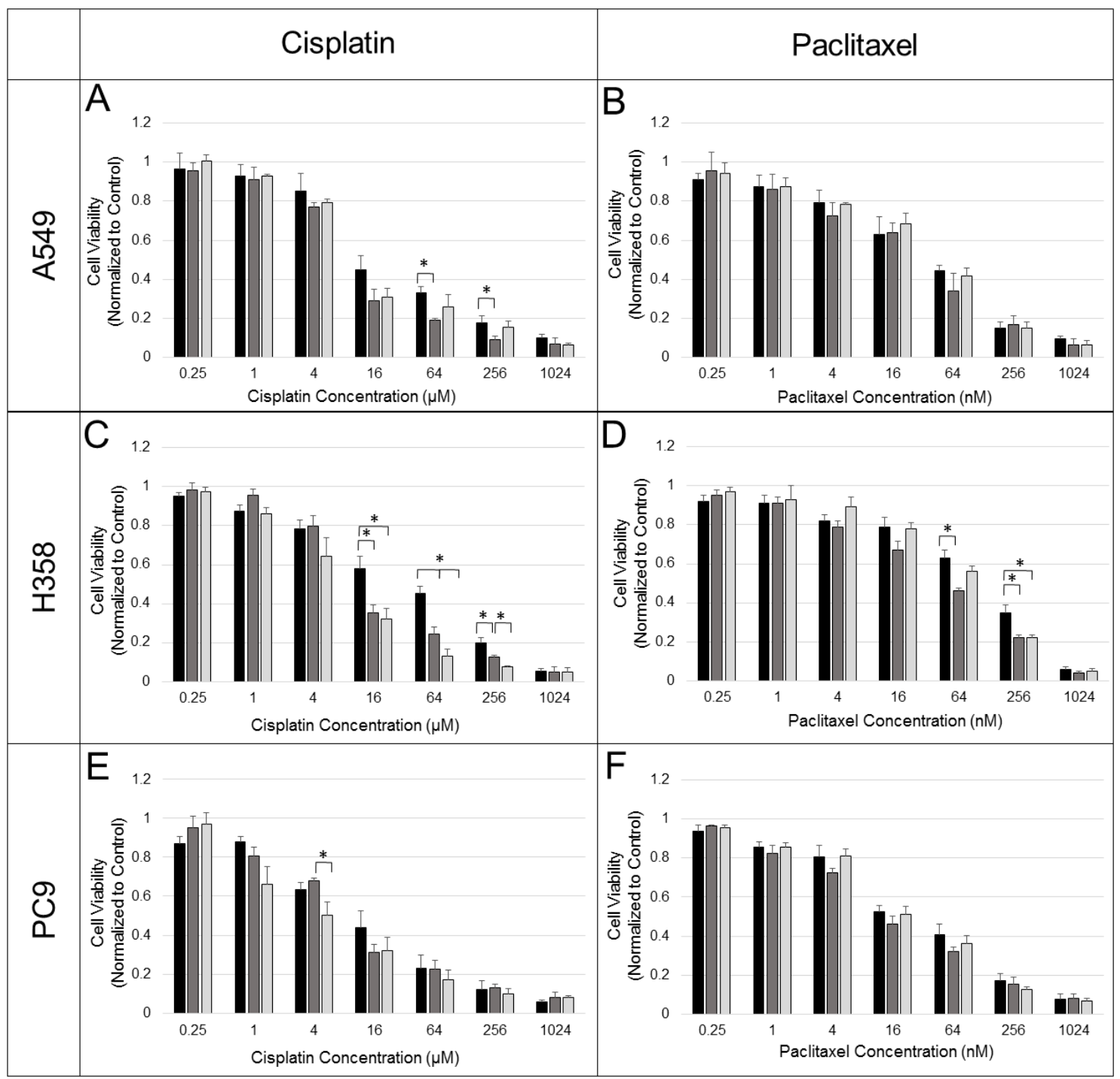

Figure 32. Cytotoxicity of drug-loaded nanoparticles in comparison to free drug in 3D cell cultures. For reference, orange bars represent free drug in 3D cell cultures, green bars represent the two-layer nanoparticle system, and dark blue represents the three layer nanoparticle system with cisplatin toxicity being shown on the left column and paclitaxel toxicity on the right column in 3 NSCLC cell lines (A549, H358, and PC9). In each case, the drug loaded nanoparticles showed similar efficacy to free drug. 


\section{Discussion}

This study examined the possible use of two and three layer nanoparticles for the delivery of both hydrophobic and hydrophilic chemotherapeutics to lung cancer. More specifically, cisplatin and paclitaxel were chosen due to their current effectiveness in treating NSCLC. To determine the effectiveness of two and three layer nanoparticles at destroying cancerous lesions, these layered nanoparticles systems were studied in both 2D (i.e. monolayer) and 3D cell cultures. Previous studies have determined that 3D cell provide a more realistic morphology of cancerous tumors that are found within the body as they possess similar characteristics [442].

The layering system was applied to the surface of gold nanoparticles to enhance their tumor targeting capabilities, while decreasing possibly immunogenicity and possibly avoid the reticuloendothelial system (RES) [443]. The RES includes macrophages of the liver and other areas noted for removing a large portion of systemically injected nanoparticles in vivo [444]. Two layer nanoparticles were synthesized with a TL layer followed by a PC layer, which was critical for the formation of a hydrophobic region capable of loading water-insoluble drugs. This was accomplished as the tail groups of TL and PC bind tail-to-tail creating a water-soluble nanoparticle with similar characteristics to liposomal delivery systems (Figure 27). With this nanoparticle system, the region between the TL and PC acts as a hydrophobic bilayer allowing for the loading of water-insoluble compounds. As liposomes cause less toxicity in vivo in comparison to other nanoparticle platforms, the PC layer also acted as a camouflage to decrease possible immunogenicity and increase bioavailability [445]. The three layer nanoparticles were constructed by adding an additional layer of HDL to the surface of two layer nanoparticles, as shown in Figure 27.

While both cisplatin and paclitaxel are commonly used in combination therapy for lung cancer, both compounds work quite differently in causing cell death and possess distinct adverse effects. While cisplatin is a water-soluble drug capable systemic delivery in saline with 5\% dextrose, paclitaxel is a hydrophobic compound that requires castor oil [446]. Castor oil has been linked to severe toxicity in patients receiving this drug [446]. For this reason, nanoparticles capable of delivering paclitaxel are of particular interest. In this study, paclitaxel is loaded into the hydrophobic region of nanoparticles formed by the TL and PC layers. With paclitaxel being loaded in this region, it was expected that paclitaxel would exhibit a slower release from the polymeric systems. This was confirmed through drug release experiments. 
Cisplatin was expected to experience a faster drug release profile due to its location of binding, onto the outer layer of PC for two layer nanoparticles or HDL for three layer nanoparticles. This faster release was attributed to the weak non-covalent interactions made between the cisplatin compound and its binding location.

After synthesis of two and three layer nanoparticles systems, characterization protocols were utilized to ensure correct modifications were present. While there is no standard set of characterization procedures at this time, we chose to use the common characterization tools followed by other groups. By characterizing the size, surface charge, and surface modifications, the identity of the nanoparticle systems could be confirmed. The wavelength of both two and three layer nanoparticles was within the common range for citrate gold nanoparticles between 50-80 nm (Figure 27) [313]. Nanoparticle size was measured using two different methods, both SEM and DLS (Figure 27 and Table 5). While sizing of nanoparticles with SEM occurs optically, measurements of size with DLS take into account the effects of Brownian motion that the particles may experience while traveling in solution. Sizing measurements by SEM and DLS can vary with nanoparticles experiencing a larger hydrodynamic radius through DLS in comparison to SEM measurements [447].

Zeta potential is another useful tool for characterizing nanoparticles as it provides information regarding the surface charge of nanoparticles (Table 5). The surface charge can have significant effects on nanoparticle travel inside the body, as proteins and other components of the body have zeta potentials that may attract or repel the nanoparticle. For example, it is documented that nanoparticles possessing a neutral charge are less likely to be removed by the liver and spleen in comparison to highly cationic or anionic nanoparticles $[448,449]$. The two layer nanoparticles with an outer layer of PC were expected to produce a slight negative surface charge. Previously, it was shown that zeta potential measurements vary with the $\mathrm{pH}$ of the solution that nanoparticle, with more acidic environments $(\mathrm{pH} \sim 5)$ producing a more positively charged surface area, while neutral $\mathrm{pH}(\sim 7)$ produced nanoparticles with negative surface charges [450]. In comparison, HDL nanoparticles were expected to possess a neutral surface charge, as the HDL molecule is relatively neutral as well [332].

Furthermore, it was critical to ensure that nanoparticles were functionalized correctly by analyzing the chemical moieties on the surface. FTIR was utilized to ensure the presence of PC and HDL on the 
surface of the gold nanoparticles (Figure 30). This was accomplished by comparing the representative peaks of PC and HDL with spectra of gold nanoparticles and pure PC and HDL. Since nanoparticles were constructed using a layering process, it was expected that some bands would be seen in both the two and three layer nanoparticle systems. The amide bond stretches between $1500-1700 \mathrm{~cm}^{-1}$ and lipid ester linkage near $1700-1800 \mathrm{~cm}^{-1}$ were the primary two bands that confirmed the presence of HDL [333].

Release of cisplatin and paclitaxel from nanoparticles was measured for 96 hours to determine the necessary doses for cytotoxicity experiments (Table 6). Cisplatin-loaded nanoparticles displayed an initial burst of drug release, a common occurrence for compounds possessing weak interactions with the nanoparticle surface. Paclitaxel-loaded nanoparticles provided a steady release of drug during the 96 hours of release, which was attributed to the efficiency of loading within the hydrophobic layer. In both cases, addition of HDL augmented the total amount of drug released during the experiment. We hypothesized that this was due to a disturbance in the PC-coating caused by the HDL components. A previous study by Scherphof et al. showed that albumin-loaded liposomes exposed to HDL would effectively release most of their payload as the HDL components disrupted the membrane integrity of the liposome [409]. This also provides evidence that the HDL components may be integrating itself into the PC layer and not creating an actual "three layer" particle.

Previous studies have shown that 3D nanoparticles are superior to 2D cell cultures for cytotoxicity experiments [421, 451]. Several companies have developed newer and easier methodologies for creating and utilizing $3 \mathrm{D}$ cell cultures in the laboratory $[424,451]$. The 3-dimensional morphology of 3D cell cultures promotes a spherical shape, in which only the cells residing on the periphery of the spheroid are exposed to the environment. When both 2D and 3D cell cultures are treated with drugs, differences in cytotoxicity become visible. In monolayer cell cultures, the cells produce a single layer allowing ensuring that the majority of will be exposed to drug. This does not hold true for 3D cell cultures as only cells of the outer periphery are exposed to drug, with inner regions of the spheroid experiencing lower levels of chemotherapeutic and possible necrosis [452]. For this reason, higher concentrations of chemotherapeutics are required to actively kill the cells residing within the center regions of the spheroid (Figure 31). Cytotoxicity experiments showed that two and three layer gold nanoparticles showed similar efficacy to free drug in all cases (Figure 32). In some instances, drug-loaded nanoparticles outperformed the free drug. 
We hypothesize the effectiveness of the nanoparticle systems can be attributed to their ability diffuse throughout the tissue gaining access to cells residing within inner regions of the spheroid. While drug molecules can diffuse from the surface of spheroid inward, diffusion limitations only permit short distances of travel for free drug, as most drug will be uptaken by the cells on the periphery of the spheroid. Previously, two- and three layer nanoparticles were shown to diffuse from the periphery of 3D cell cultures inward more effectively than previously designed PEGylated particles [225]. As the nanoparticles are constantly releasing drug as they diffuse from the periphery of the spheroid inward, the cells being exposed are receiving adequate concentrations of drug effective for cellular apoptosis.

This study analyzed the cytotoxicity of two and three layer gold nanoparticles in 3D cell cultures to determine the possible applications for in vivo work in the future. As the particles showed similar efficacy to the freely drug, we would expect that the nanoparticles would function well in vivo as the nanoparticles are more likely to accumulate in the tumor as compared to freely circulating drug. Results from this study also support the possibility of attaching both paclitaxel and cisplatin a single nanoparticle, allowing for a combination treatment in the future. 


\section{CHAPTER VI}

\section{SUMMARY AND CONCLUSIONS}

The purpose of this project was to examine the use of drug loaded gold nanoparticles as potential drug delivery agents for the treatment of solid tumors. This was accomplished by analyzing the diffusivity of gold nanoparticles in 3D cell cultures, measuring the uptake of gold nanoparticles in vivo when injected systemically, interpreting the drug-loading capabilities of functionalized gold nanoparticles, and to determine the efficacy of these drug-loaded gold nanoparticles in 2D and 3D cell cultures.

The ability to modify the surface of nanoparticles makes them well adaptable for several applications including the delivery of chemotherapeutics to solid tumors. This project specifically utilized nanoparticles modified using a layering process, in which surface functionalization of thiol and PC created two layer nanoparticles, and an additional layer of HDL onto the PC layer formed the three layer nanoparticles. It was hypothesized that the addition of PC would decrease possible immunogenicity and immune cell uptake in various filtering organs in the body. As HDL is up-regulated in some forms of cancer, it was predicted that three layer nanoparticles might have additional benefits from the PC-coated nanoparticles.

Two and three layer citrate gold nanoparticles and silica gold nanoshells were synthesized. While both citrate gold nanoparticles and silica gold nanoshells are both metallic nanostructures, they differ in size and applications. Synthesis of nanoparticles and accurate modifications were confirmed using several characterization techniques described in the methods section. It was also predicted that PC-coated nanoparticles would be able to diffuse greater distances from vasculature due to its similar structural composition in comparison to cellular membranes. For comparison, previously developed PEGylated nanoparticles were utilized. Our hypotheses were confirmed in this study as the two and three layer nanoparticles diffused further from vasculature in comparison to PEGylated nanoparticles, suggesting that PC- and HDL- coated nanoparticles may potentially be able to delivery drug deeper into tumors. In addition, this study showed that smaller citrate gold nanoparticles accumulated in 3D cell cultures at higher 
concentrations in comparison to silica gold nanoshells. In the pancreatic cancer cell line, S2VP10, the two layer nanoparticles showed superior diffusivity in comparison to PEGylated and HDL-coated nanoparticles. For this reason, two layer citrate gold nanoparticles and silica gold nanoshells were used for in vivo experimentation.

After analyzing the diffusivity of gold nanoparticles in 3D cell cultures, the two layer nanoparticles were examined in vivo. Mice with orthotopic pancreatic adenocarcinomas were injected with two layer gold nanoparticles to determine the accumulation efficiency in the tumor vs. filtering organs (i.e. liver and spleen). The diffusivities of the nanoparticles from vasculature were also measured to determine which particle could more effectively exit the fenestrations of the solid tumor. This was accomplished using dark field microscopy in combination with hyperspectral imaging, using the CytoViva Hyperspectral Imaging system. Results from this study showed a size-dependent hindrance of nanoparticle diffusion, as the PC-coated silica gold nanoshells diffused shorter distances from vasculature in comparison to smaller citrate gold nanoparticles. As the size difference is $3: 1$, this was expected. An overall accumulation of colloidal gold nanoparticles was higher in the tumor, liver, and spleen in comparison to silica gold nanoshells. Since citrate gold nanoparticles showed superior efficacy compared to silica gold nanoshells, the smaller nanoparticles were chosen for the remainder of this project for drug loading and in vitro experimentation.

Since the colloidal gold nanoparticles displayed higher accumulation in vivo, they were utilized to determine drug loading capabilities and release kinetics. Drug release from nanoparticles is a complex process dependent upon the physiochemical properties of the nanoparticle and drug molecule. Since the nanoparticles were layered, the TL and PC surface modifications created a region of hydrophobicity around the nanoparticle optimal for loading the water-insoluble compound paclitaxel. On the outside of the nanoparticles, cisplatin was loaded and interacted through non-covalent interactions. Results from this study showed that paclitaxel released slower than cisplatin, which was attributed to method of drug loading. The addition of the third layer (HDL) was shown to puncture the PC layer of the nanoparticles, promoting the release of more paclitaxel from the hydrophobic layer. By understanding the amount of drug released at various time intervals, we were able to design cytotoxicity experiments to test the efficacy of these novel nanotherapeutic systems. 
The efficacy of cisplatin and paclitaxel-loaded colloidal gold nanoparticles were evaluated in 3D cell cultures. Results from this study showed that drug-loaded nanoparticles performed similarly to free drug in each case. As the nanoparticles are targeted to tumors, unlike freely circulating drug, we would expect the nanoparticles to perform well in an animal model. Also, nanoparticles could result in less liver and kidney toxicity as drug release is delayed from nanoparticle systems, unlike free drug, which accumulates in the liver faster.

In conclusion, the two and three layer nanoparticles system is a potential candidate for cancer therapy. Additional studies are needed to determine the effectiveness of the nanoparticle compounds in animal models. Future prospects for this project include the co-delivery of two drugs simultaneously. Additionally, more studies are needed to examine potential toxicities associated with both two and three layer nanoparticles. 


\section{REFERENCES}

1. Cairns, R., I. Papandreou, and N. Denko, Overcoming physiologic barriers to cancer treatment by molecularly targeting the tumor microenvironment. Mol Cancer Res, 2006. 4(2): p. 61-70.

2. Waite, C.L. and C.M. Roth, Nanoscale drug delivery systems for enhanced drug penetration into solid tumors: current progress and opportunities. Crit Rev Biomed Eng, 2012. 40(1): p. 21-41.

3. Szala, S. and M. Jarosz, [Tumor blood vessels]. Postepy Hig Med Dosw (Online), 2011. 65: p. 437-46.

4. Minchinton, A.I. and I.F. Tannock, Drug penetration in solid tumours. Nat Rev Cancer, 2006. 6(8): p. 583-92.

5. Izuishi, K., et al., Remarkable tolerance of tumor cells to nutrient deprivation: possible new biochemical target for cancer therapy. Cancer Res, 2000. 60(21): p. 6201-7.

6. Heldin, C.H., et al., High interstitial fluid pressure - an obstacle in cancer therapy. Nat Rev Cancer, 2004. 4(10): p. 806-13.

7. Hofmann, M., et al., Lowering of tumor interstitial fluid pressure reduces tumor cell proliferation in a xenograft tumor model. Neoplasia, 2006. 8(2): p. 89-95.

8. Curti, B.D., et al., Interstitial pressure of subcutaneous nodules in melanoma and lymphoma patients: changes during treatment. Cancer Res, 1993. 53(10 Suppl): p. 2204-7.

9. Hirst, D.G. and J. Denekamp, Tumour cell proliferation in relation to the vasculature. Cell Tissue Kinet, 1979. 12(1): p. 31-42.

10. Moulder, J.E. and S. Rockwell, Tumor hypoxia: its impact on cancer therapy. Cancer Metastasis Rev, 1987. 5(4): p. 313-41.

11. Dean, M., T. Fojo, and S. Bates, Tumour stem cells and drug resistance. Nat Rev Cancer, 2005. 5(4): p. $275-84$.

12. Vandenberg, A.P., et al., Tumor Ph in Human Mammary-Carcinoma. European Journal of Cancer \& Clinical Oncology, 1982. 18(5): p. 457-462. 
13. Mahoney, B.P., et al., Tumor acidity, ion trapping and chemotherapeutics. I. Acid pH affects the distribution of chemotherapeutic agents in vitro. Biochem Pharmacol, 2003. 66(7): p. 1207-18.

14. Stylianopoulos, T., et al., Diffusion of Particles in the Extracellular Matrix: The Effect of Repulsive Electrostatic Interactions. Biophysical Journal, 2010. 99(5): p. 1342-1349.

15. Ma, P. and R.J. Mumper, Paclitaxel Nano-Delivery Systems: A Comprehensive Review. J Nanomed Nanotechnol, 2013. 4(2): p. 1000164.

16. Gupta, N., H. Hatoum, and G.K. Dy, First line treatment of advanced non-small-cell lung cancer specific focus on albumin bound paclitaxel. Int J Nanomedicine, 2014. 9: p. 209-221.

17. Kolhar, P., et al., Using shape effects to target antibody-coated nanoparticles to lung and brain endothelium. Proceedings of the National Academy of Sciences of the United States of America, 2013. 110(26): p. 10753-10758.

18. Taruttis, A., et al., Real-time imaging of cardiovascular dynamics and circulating gold nanorods with multispectral optoacoustic tomography. Optics Express, 2010. 18(19): p. 19592-19602.

19. Ren, F., et al., Gold Nanorods Carrying Paclitaxel for Photothermal-Chemotherapy of Cancer. Bioconjugate Chemistry, 2013. 24(3): p. 376-386.

20. Thurston, G., et al., Cationic liposomes target angiogenic endothelial cells in tumors and chronic inflammation in mice. Journal of Clinical Investigation, 1998. 101(7): p. 1401-1413.

21. Krasnici, S., et al., Effect of the surface charge of liposomes on their uptake by angiogenic tumor vessels. International Journal of Cancer, 2003. 105(4): p. 561-567.

22. Lieleg, O., R.M. Baumgartel, and A.R. Bausch, Selective Filtering of Particles by the Extracellular Matrix: An Electrostatic Bandpass. Biophysical Journal, 2009. 97(6): p. 1569-1577.

23. Unezaki, S., et al., Direct measurement of the extravasation of polyethyleneglycol-coated liposomes into solid tumor tissue by in vivo fluorescence microscopy. International Journal of Pharmaceutics, 1996. 144(1): p. 11-17.

24. Huang, K., et al., Size-dependent localization and penetration of ultrasmall gold nanoparticles in cancer cells, multicellular spheroids, and tumors in vivo. ACS Nano, 2012. 6(5): p. 4483-93.

25. Kao, H.W., et al., Evaluation of EGFR-targeted radioimmuno-gold-nanoparticles as a theranostic agent in a tumor animal model. Bioorg Med Chem Lett, 2013. 23(11): p. 3180-5. 
26. Creixell, M., et al., EGFR-Targeted Magnetic Nanoparticle Heaters Kill Cancer Cells without a Perceptible Temperature Rise. Acs Nano, 2011. 5(9): p. 7124-7129.

27. Milane, L., Z.F. Duan, and M. Amiji, Pharmacokinetics and biodistribution of lonidamine/paclitaxel loaded, EGFR-targeted nanoparticles in an orthotopic animal model of multi-drug resistant breast cancer. Nanomedicine, 2011. 7(4): p. 435-44.

28. Peng, X.H., et al., Targeted Delivery of Cisplatin to Lung Cancer Using ScFvEGFR-Heparin-Cisplatin Nanoparticles. Acs Nano, 2011. 5(12): p. 9480-9493.

29. Rangger, C., et al., Tumor targeting and imaging with dual-peptide conjugated multifunctional liposomal nanoparticles. International Journal of Nanomedicine, 2013. 8: p. 4659-4670.

30. Konno, T., et al., Effect of Arterial Administration of High-Molecular-Weight Anti-Cancer Agent Smancs with Lipid Lymphographic Agent on Hepatoma - a Preliminary-Report. European Journal of Cancer \& Clinical Oncology, 1983. 19(8): p. 1053-\&.

31. Litzinger, D.C., et al., Effect of Liposome Size on the Circulation Time and Intraorgan Distribution of Amphipathic Poly(Ethylene Glycol)-Containing Liposomes. Biochimica Et Biophysica ActaBiomembranes, 1994. 1190(1): p. 99-107.

32. Hong, M.H., et al., Efficient tumor targeting of hydroxycamptothecin loaded PEGylated niosomes modified with transferrin. Journal of Controlled Release, 2009. 133(2): p. 96-102.

33. Greish, K., Enhanced permeability and retention of macromolecular drugs in solid tumors: A royal gate for targeted anticancer nanomedicines. Journal of Drug Targeting, 2007. 15(7-8): p. 457-464.

34. Maeda, H., et al., Conjugation of Poly(Styrene-Co-Maleic Acid) Derivatives to the Antitumor Protein Neocarzinostatin - Pronounced Improvements in Pharmacological Properties. Journal of Medicinal Chemistry, 1985. 28(4): p. 455-461.

35. Matsumura, Y. and H. Maeda, A New Concept for Macromolecular Therapeutics in CancerChemotherapy - Mechanism of Tumoritropic Accumulation of Proteins and the Antitumor Agent Smancs. Cancer Research, 1986. 46(12): p. 6387-6392.

36. Merisko-Liversidge, E.M. and G.G. Liversidge, Drug nanoparticles: formulating poorly water-soluble compounds. Toxicol Pathol, 2008. 36(1): p. 43-8.

37. Gradishar, W.J., Albumin-bound paclitaxel: a next-generation taxane. Expert Opin Pharmacother, 2006. 7(8): p. 1041-53.

38. Yin, H., H. Zhang, and B. Liu, Superior anticancer efficacy of curcumin-loaded nanoparticles against lung cancer. Acta Biochim Biophys Sin (Shanghai), 2013. 45(8): p. 634-40. 
39. Zhang, Z. and S.S. Feng, Nanoparticles of poly(lactide)/vitamin E TPGS copolymer for cancer chemotherapy: synthesis, formulation, characterization and in vitro drug release. Biomaterials, 2006. 27(2): p. 262-70.

40. Li, M., et al., Nanoscaled poly(L-glutamic acid)/doxorubicin-amphiphile complex as $p H$-responsive drug delivery system for effective treatment of nonsmall cell lung cancer. ACS Appl Mater Interfaces, 2013. 5(5): p. 1781-92.

41. Taruttis, A., et al., Real-time imaging of cardiovascular dynamics and circulating gold nanorods with multispectral optoacoustic tomography. Opt Express, 2010. 18(19): p. 19592-602.

42. Ma, R., et al., Multispectral optoacoustic tomography (MSOT) scanner for whole-body small animal imaging. Optics Express, 2009. 17(24): p. 21414-21426.

43. Ntziachristos, V. and D. Razansky, Molecular imaging by means of multispectral optoacoustic tomography (MSOT). Chem Rev, 2010. 110(5): p. 2783-94.

44. Patel, A.R., et al., Theranostic tumor homing nanocarriers for the treatment of lung cancer. Nanomedicine, 2013.

45. Taratula, O., et al., Innovative strategy for treatment of lung cancer: targeted nanotechnology-based inhalation co-delivery of anticancer drugs and siRNA. J Drug Target, 2011. 19(10): p. 900-14.

46. Liu, Q., et al., Delivering hydrophilic and hydrophobic chemotherapeutics simultaneously by magnetic mesoporous silica nanoparticles to inhibit cancer cells. Int J Nanomedicine, 2012. 7: p. 999-1013.

47. Cancer multidrug resistance. Nat Biotech, 2000.

48. Patel, N.R., et al., Nanopreparations to overcome multidrug resistance in cancer. Advanced Drug Delivery Reviews, 2013. 65(13-14): p. 1748-1762.

49. Grant, C.E., et al., Overexpression of Multidrug Resistance-Associated Protein (Mrp) Increases Resistance to Natural Product Drugs. Cancer Research, 1994. 54(2): p. 357-361.

50. Allen, J.D., et al., Extensive contribution of the multidrug transporters P-glycoprotein and Mrp1 to basal drug resistance. Cancer Research, 2000. 60(20): p. 5761-5766.

51. Cuvier, C., et al., Doxorubicin-loaded nanospheres bypass tumor cell multidrug resistance. Biochemical Pharmacology, 1992. 44(3): p. 509-517. 
52. Beh, C.W., et al., Efficient Delivery of Bcl-2-Targeted siRNA Using Cationic Polymer Nanoparticles: Downregulating mRNA Expression Level and Sensitizing Cancer Cells to Anticancer Drug. Biomacromolecules, 2009. 10(1): p. 41-48.

53. Chen, A.M., et al., Co-delivery of Doxorubicin and Bcl-2 siRNA by Mesoporous Silica Nanoparticles Enhances the Efficacy of Chemotherapy in Multidrug-Resistant Cancer Cells. Small, 2009. 5(23): p. 2673-2677.

54. Cheng, D., et al., Multifunctional nanocarrier mediated co-delivery of doxorubicin and siRNA for synergistic enhancement of glioma apoptosis in rat. Biomaterials, 2012. 33(4): p. 1170-1179.

55. Saad, M., O.B. Garbuzenko, and T. Minko, Co-delivery of siRNA and an anticancer drug for treatment of multidrug-resistant cancer. Nanomedicine (Lond), 2008. 3(6): p. 761-76.

56. Society, A.C., Cancer Facts \& Figures 2013, 2013, American Cancer Society: Atlanta.

57. Group, U.S.C.S.W. United States Cancer Statistics: 1999-2010 Incidence and Mortality Web-based Report. 2013; Available from: www.cdc.gov/uscs.

58. Hong, W.K., Holland Frei Cancer Medicine 8. 8th ed. Vol. 8. 2010, Shelton, Conn.: People's Medical Pub. House.

59. Jemal, A., M.M. Center, and E. Ward, The convergence of lung cancer rates between blacks and whites under the age of 40, United States. Cancer Epidemiol Biomarkers Prev, 2009. 18(12): p. 3349-52.

60. Doll, R. and A.B. Hill, Smoking and carcinoma of the lung; preliminary report. Br Med J, 1950. 2(4682): p. 739-48.

61. Brambilla, E., et al., The new World Health Organization classification of lung tumours. Eur Respir J, 2001. 18(6): p. 1059-68.

62. Alberg, A.J. and J.M. Samet, Epidemiology of lung cancer. Chest, 2003. 123(1 Suppl): p. 21S-49S.

63. Koop, C.E. and J. Luoto, "The health consequences of smoking: cancer," overview of a report of the Surgeon General. 1982. Public Health Rep, 2006. 121 Suppl 1: p. 269-75; discussion 268.

64. Halpern, M.T., B.W. Gillespie, and K.E. Warner, Patterns of absolute risk of lung cancer mortality in former smokers. J Natl Cancer Inst, 1993. 85(6): p. 457-64.

65. Saracci, R., The interaction of tobacco smoking and other agents in cancer etiology. Epidemiologic Reviews, 1987. 9(1): p. 175-193. 
66. De Matteis S, C.D., Bertazzi PA, Exposure to occupational carcinogens and lung cancer risk.

Evolution of epidemiological estimates of attributable fraction. Acta Biomed . 2008. 79: p. 34-42.

67. Doll, R. and R. Peto, The causes of cancer: quantitative estimates of avoidable risks of cancer in the United States today. J Natl Cancer Inst, 1981. 66(6): p. 1191-308.

68. Inskip, P.D., M. Stovall, and J.T. Flannery, Lung cancer risk and radiation dose among women treated for breast cancer. J Natl Cancer Inst, 1994. 86(13): p. 983-8.

69. Arai, T., et al., Tumor Doubling Time and Prognosis in Lung Cancer Patients: Evaluation from Chest Films and Clinical Follow-up Study. Japanese Journal of Clinical Oncology, 1994. 24(4): p. 199204.

70. Sher, T., G.K. Dy, and A.A. Adjei, Small cell lung cancer. Mayo Clin Proc, 2008. 83(3): p. 355-67.

71. Kerr, K.M., Pulmonary adenocarcinomas: classification and reporting. Histopathology, 2009. 54(1): p. 12-27.

72. Subramanian, J. and R. Govindan, Lung cancer in never smokers: a review. J Clin Oncol, 2007. 25(5): p. 561-70.

73. Ettinger, D.S., et al., Non-small cell lung cancer. J Natl Compr Canc Netw, 2010. 8(7): p. 740-801.

74. Perez-Moreno, P., et al., Squamous cell carcinoma of the lung: molecular subtypes and therapeutic opportunities. Clin Cancer Res, 2012. 18(9): p. 2443-51.

75. Đurić Milivoje, M.-Đ.M., Gajić Dobrica, Damjanović Goran, Cutaneous metastasis as the first sign of lung cancer. Archive of Oncology, 2013. 21(1): p. 20-22.

76. Kalemkerian, G.P., Staging and imaging of small cell lung cancer. Cancer Imaging, 2011. 11: p. 253-8.

77. Jett, J.R. and D.E. Midthun, Screening for lung cancer: current status and future directions: Thomas A. Neff lecture. Chest, 2004. 125(5 Suppl): p. 158S-62S.

78. Oken, M.M., et al., Screening by chest radiograph and lung cancer mortality: the Prostate, Lung, Colorectal, and Ovarian (PLCO) randomized trial. JAMA, 2011. 306(17): p. 1865-73.

79. Nolop, K.B., et al., Glucose utilization in vivo by human pulmonary neoplasms. Cancer, 1987. 60(11): p. 2682-9. 
80. Silvestri, G.A., et al., Noninvasive staging of non-small cell lung cancer: ACCP evidenced-based clinical practice guidelines (2nd edition). Chest, 2007. 132(3 Suppl): p. 178S-201S.

81. Sox, H.C., Better Evidence about Screening for Lung Cancer. New England Journal of Medicine, 2011. 365(5): p. 455-457.

82. Yung, R.C., Tissue diagnosis of suspected lung cancer: selecting between bronchoscopy, transthoracic needle aspiration, and resectional biopsy. Respir Care Clin N Am, 2003. 9(1): p. 51-76.

83. Mountain, C.F. and C.M. Dresler, Regional lymph node classification for lung cancer staging. Chest, 1997. 111(6): p. 1718-1723.

84. Mountain, C.F., A New International Staging System for Lung-Cancer. Chest, 1986. 89(4): p. S225S233.

85. Mountain, C.F., Revisions in the international system for staging lung cancer. CHEST Journal, 1997. 111(6): p. 1710-1717.

86. Health, N.I.o. Cancer Drug Information: Cisplatin. 2014 01/25/2014].

87. Spira, A. and D.S. Ettinger, Multidisciplinary management of lung cancer. N Engl J Med, 2004. 350(4): p. 379-92.

88. Le Chevalier, T., et al., Cisplatin-based adjuvant chemotherapy in patients with completely resected non-small-cell lung cancer. New England Journal of Medicine, 2004. 350(4): p. 351-360.

89. Chemotherapy in non-small cell lung cancer: a meta-analysis using updated data on individual patients from 52 randomised clinical trials. Non-small Cell Lung Cancer Collaborative Group. BMJ, 1995. 311(7010): p. 899-909.

90. Network, N.C.C., NCCN Clinical Practice Guidelines in Oncology-Non-Small Cell Lung Cancer. 2007.

91. Dipiro J, T.R., Yee G, Matzke G, Wells B, Michael Posey, Pharmacotherapy: A Pathophysiologic Approach. Vol. 7. 2008: McGraw Hill Medical. 2157-2173.

92. Kris, M.G., et al., Efficacy of gefitinib, an inhibitor of the epidermal growth factor receptor tyrosine kinase, in symptomatic patients with non-small cell lung cancer: a randomized trial. JAMA, 2003. 290(16): p. 2149-58. 
93. Lee, S.M., et al., First-line erlotinib in patients with advanced non-small-cell lung cancer unsuitable for chemotherapy (TOPICAL): a double-blind, placebo-controlled, phase 3 trial. Lancet Oncol, 2012. 13(11): p. 1161-70.

94. Network, N.C.C., NCCN Clincal Practive Guidelines in Oncology-Small Cell Lung Cancer. 2007.

95. Mu, Q., D.L. Broughton, and B. Yan, Endosomal leakage and nuclear translocation of multiwalled carbon nanotubes: developing a model for cell uptake. Nano Lett, 2009. 9(12): p. 4370-5.

96. Bianco, A., K. Kostarelos, and M. Prato, Applications of carbon nanotubes in drug delivery. Curr Opin Chem Biol, 2005. 9(6): p. 674-9.

97. Carbon nanotubes may prove to be highly effective vectors for siRNA treatment in the fight against lung cancer. Nanomedicine, 2009. 4(4): p. 377-378.

98. Lu, X.K., et al., Tailoring graphite with the goal of achieving single sheets. Nanotechnology, 1999. 10(3): p. 269-272.

99. He, H., et al., Carbon nanotubes: applications in pharmacy and medicine. Biomed Res Int, 2013. 2013: p. 578290.

100. Chen, J., et al., In vitro and in vivo gene delivery using polyethylenimine-poly(hydroxyethyl glutamine) as a non-viral carrier. J Control Release, 2011. 152 Suppl 1: p. e134-6.

101. Sargent, L.M., et al., Single-walled carbon nanotube-induced mitotic disruption. Mutat Res, 2012. 745(1-2): p. 28-37.

102. Wang, L., et al., Carbon nanotubes induce malignant transformation and tumorigenesis of human lung epithelial cells. Nano Lett, 2011. 11(7): p. 2796-803.

103. Lohcharoenkal, W., et al., Chronic Exposure to Carbon Nanotubes Induces Invasion of Human Mesothelial Cells through Matrix Metalloproteinase-2. ACS Nano, 2013.

104. Das, M., et al., Augmented Anticancer Activity of a Targeted, Intracellularly Activatable, Theranostic Nanomedicine Based on Fluorescent and Radiolabeled, Methotrexate-Folic Acid-Multiwalled Carbon Nanotube Conjugate. Mol Pharm, 2013.

105. Datir, S.R., et al., Hyaluronate tethered, "smart" multiwalled carbon nanotubes for tumor-targeted delivery of doxorubicin. Bioconjug Chem, 2012. 23(11): p. 2201-13. 
106. Arya, N., et al., Combination of single walled carbon nanotubes/graphene oxide with paclitaxel: a reactive oxygen species mediated synergism for treatment of lung cancer. Nanoscale, 2013. 5(7): p. 2818-2829.

107. Minati, L., et al., Multifunctional Branched Gold-Carbon Nanotube Hybrid for Cell Imaging and Drug Delivery. Langmuir, 2012. 28(45): p. 15900-15906.

108. Srivastava, R.K., et al., Multi-walled carbon nanotubes induce oxidative stress and apoptosis in human lung cancer cell line-A549. Nanotoxicology, 2011. 5(2): p. 195-207.

109. Ohnishi, M., et al., Novel method using hybrid markers: development of an approach for pulmonary measurement of multi-walled carbon nanotubes. Journal of Occupational Medicine and Toxicology, 2013. 8.

110. Pacurari, M., et al., Multi-walled carbon nanotube-induced gene expression in the mouse lung: Association with lung pathology. Toxicology and Applied Pharmacology, 2011. 255(1): p. 18-31.

111. Stella, G.M., Carbon nanotubes and pleural damage: Perspectives of nanosafety in the light of asbestos experience. Biointerphases, 2011. 6(2): p. P1-P17.

112. Haniu, H., et al., Proteomics-based safety evaluation of multi-walled carbon nanotubes. Toxicology and Applied Pharmacology, 2010. 242(3): p. 256-262.

113. Sargent, L.M., et al., Induction of Aneuploidy by Single-Walled Carbon Nanotubes. Environmental and Molecular Mutagenesis, 2009. 50(8): p. 708-717.

114. Folkmann, J.K., et al., Oxidatively Damaged DNA in Rats Exposed by Oral Gavage to C-60 Fullerenes and Single-Walled Carbon Nanotubes. Environmental Health Perspectives, 2009. 117(5): p. 703-708.

115. Alivisatos, A.P., Perspectives on the physical chemistry of semiconductor nanocrystals. Journal of Physical Chemistry, 1996. 100(31): p. 13226-13239.

116. Fogg, D.E., et al., Fabrication of quantum dot-polymer composites: Semiconductor nanoclusters in dual-function polymer matrices with electron-transporting and cluster-passivating properties. Macromolecules, 1997. 30(26): p. 8433-8439.

117. Dabbousi, B.O., et al., (CdSe)ZnS core-shell quantum dots: Synthesis and characterization of a size series of highly luminescent nanocrystallites. Journal of Physical Chemistry B, 1997. 101(46): p. 9463-9475.

118. Kuno, M., et al., The band edge luminescence of surface modified CdSe nanocrystallites: Probing the luminescing state. Journal of Chemical Physics, 1997. 106(23): p. 9869-9882. 
119. RodriguezViejo, J., et al., Cathodoluminescence and photoluminescence of highly luminescent CdSe/ZnS quantum dot composites. Applied Physics Letters, 1997. 70(16): p. 2132-2134.

120. Zhang, H., D. Yee, and C. Wang, Quantum dots for cancer diagnosis and therapy: biological and clinical perspectives. Nanomedicine (Lond), 2008. 3(1): p. 83-91.

121. Li, Y.F. and C.Y. Chen, Fate and Toxicity of Metallic and Metal-Containing Nanoparticles for Biomedical Applications. Small, 2011. 7(21): p. 2965-2980.

122. Choi, Y.J., et al., Cyto-/genotoxic effect of CdSe/ZnS quantum dots in human lung adenocarcinoma cells for potential photodynamic UV therapy applications. J Nanosci Nanotechnol, 2012. 12(3): p. 2160-8.

123. Wang, Y., et al., Detection of micrometastases in lung cancer with magnetic nanoparticles and quantum dots. Int J Nanomedicine, 2012. 7: p. 2315-24.

124. Li, H., et al., Effects of Paclitaxel on EGFR Endocytic Trafficking Revealed Using Quantum Dot Tracking in Single Cells. Plos One, 2012. 7(9).

125. Nurunnabi, M., et al., In Vivo Biodistribution and Toxicology of Carboxylated Graphene Quantum Dots. Acs Nano, 2013. 7(8): p. 6858-6867.

126. Zhang, C.L., et al., One-Pot Synthesized Aptamer-Functionalized CdTe:Zn2+ Quantum Dots for Tumor-Targeted Fluorescence Imaging in Vitro and in Vivo. Analytical Chemistry, 2013. 85(12): p. 5843-5849.

127. Li, H., et al., Simultaneous detection of two lung cancer biomarkers using dual-color fluorescence quantum dots. Analyst, 2011. 136(7): p. 1399-405.

128. Manzoor, K., et al., Bio-conjugated luminescent quantum dots of doped ZnS: a cyto-friendly system for targeted cancer imaging. Nanotechnology, 2009. 20(6): p. 065102.

129. Song, E.Q., et al., Tumor cell targeting using folate-conjugated fluorescent quantum dots and receptor-mediated endocytosis. Clin Chem, 2009. 55(5): p. 955-63.

130. Prajapat, R.P., et al., Dendrimer: A polymer of 21st century. 2010.

131. Bharali, D.J., et al., Nanoparticles and cancer therapy: a concise review with emphasis on dendrimers. Int J Nanomedicine, 2009. 4: p. 1-7. 
132. Markman, J.L., et al., Nanomedicine therapeutic approaches to overcome cancer drug resistance. Adv Drug Deliv Rev, 2013. 65(13-14): p. 1866-79.

133. Oberoi, H.S., et al., Nanocarriers for delivery of platinum anticancer drugs. Adv Drug Deliv Rev, 2013. 65(13-14): p. 1667-85.

134. Movassaghian, S., et al., Efficient down-regulation of PKC-alpha gene expression in A549 lung cancer cells mediated by antisense oligodeoxynucleotides in dendrosomes. International Journal of Pharmaceutics, 2013. 441(1-2): p. 82-91.

135. Wang, H., et al., Folic acid-modified dendrimer-entrapped gold nanoparticles as nanoprobes for targeted CT imaging of human lung adencarcinoma. Biomaterials, 2013. 34(2): p. 470-480.

136. Liu, J.F., et al., Novel peptide-dendrimer conjugates as drug carriers for targeting nonsmall cell lung cancer. International Journal of Nanomedicine, 2011. 6: p. 59-69.

137. Malik, N., E.G. Evagorou, and R. Duncan, Dendrimer-platinate: a novel approach to cancer chemotherapy. Anti-Cancer Drugs, 1999. 10(8): p. 767-776.

138. Kurmi, B.D., et al., Lactoferrin-Conjugated Dendritic Nanoconstructs for Lung Targeting of Methotrexate. Journal of Pharmaceutical Sciences, 2011. 100(6): p. 2311-2320.

139. Reichert, S., et al., Size-Dependant Cellular Uptake of Dendritic Polyglycerol. Small, 2011. 7(6): p. 820-829.

140. Lim, J., et al., Design, Synthesis, Characterization, and Biological Evaluation of Triazine Dendrimers Bearing Paclitaxel Using Ester and Ester/Disulfide Linkages. Bioconjugate Chemistry, 2009. 20(11): p. 2154-2161.

141. Lee, J.H., et al., Nanosized polyamidoamine (PAMAM) dendrimer-induced apoptosis mediated by mitochondrial dysfunction. Toxicology Letters, 2009. 190(2): p. 202-207.

142. Zhao, P., et al., Paclitaxel-loaded, folic-acid-targeted and TAT-peptide-conjugated polymeric liposomes: in vitro and in vivo evaluation. Pharm Res, 2010. 27(9): p. 1914-26.

143. Cai, L., et al., Peptide ligand and PEG-mediated long-circulating liposome targeted to FGFR overexpressing tumor in vivo. Int J Nanomedicine, 2012. 7: p. 4499-510.

144. Yang, X.Q., et al., [nm23-H1-siRNA enhances the chemosensitivity to liposome-encapsulated paclitaxel in lung adenocarcinoma cells in vitro]. Zhonghua Zhong Liu Za Zhi, 2011. 33(6): p. 405-9. 
145. Zhou, J., et al., The anticancer efficacy of paclitaxel liposomes modified with mitochondrial targeting conjugate in resistant lung cancer. Biomaterials, 2013. 34(14): p. 3626-38.

146. Li, M., et al., Three-Dimensional Hierarchical Plasmonic Nano-Architecture Enhanced SurfaceEnhanced Raman Scattering Immunosensor for Cancer Biomarker Detection in Blood Plasma. Acs Nano, 2013. 7(6): p. 4967-4976.

147. Stathopoulos, G.P., Liposomal cisplatin: a new cisplatin formulation. Anticancer Drugs, 2010. 21(8): p. 732-6.

148. Oberoi, H.S., et al., Nanocarriers for delivery of platinum anticancer drugs. Advanced Drug Delivery Reviews, 2013. 65(13-14): p. 1667-1685.

149. Casagrande, N., et al., Preclinical evaluation of a new liposomal formulation of cisplatin, lipoplatin, to treat cisplatin-resistant cervical cancer. Gynecol Oncol, 2013. 131(3): p. 744-52.

150. Zalba, S. and M.J. Garrido, Liposomes, a promising strategy for clinical application of platinum derivatives. Expert Opin Drug Deliv, 2013. 10(6): p. 829-44.

151. Fantini, M., et al., Lipoplatin treatment in lung and breast cancer. Chemother Res Pract, 2011. 2011: p. 125192.

152. Stathopoulos, G.P. and T. Boulikas, Lipoplatin formulation review article. J Drug Deliv, 2012. 2012: p. 581363.

153. Gaspar, M.M., et al., Targeted delivery of transferrin-conjugated liposomes to an orthotopic model of lung cancer in nude rats. J Aerosol Med Pulm Drug Deliv, 2012. 25(6): p. 310-8.

154. Chang, D.K., et al., Peptide-mediated liposomal Doxorubicin enhances drug delivery efficiency and therapeutic efficacy in animal models. PLoS One, 2013. 8(12): p. e83239.

155. Cheng, C., et al., Photodynamic therapy selectively enhances liposomal doxorubicin uptake in sarcoma tumors to rodent lungs. Lasers Surg Med, 2010. 42(5): p. 391-9.

156. Chang, D.K., et al., A novel peptide enhances therapeutic efficacy of liposomal anti-cancer drugs in mice models of human lung cancer. PLoS One, 2009. 4(1): p. e4171.

157. Guo, L., et al., A novel combination of TRAIL and doxorubicin enhances antitumor effect based on passive tumor-targeting of liposomes. Nanotechnology, 2011. 22(26): p. 265105.

158. Yang, Y., et al., Systemic delivery of siRNA via LCP nanoparticle efficiently inhibits lung metastasis. Mol Ther, 2012. 20(3): p. 609-15. 
159. Taetz, S., et al., Hyaluronic acid-modified DOTAP/DOPE liposomes for the targeted delivery of antitelomerase siRNA to CD44-expressing lung cancer cells. Oligonucleotides, 2009. 19(2): p. 10316.

160. Kuznetsova, N.R., et al., Targeting liposomes loaded with melphalan prodrug to tumour vasculature via the Sialyl Lewis X selectin ligand. J Drug Target, 2013.

161. Saffari, M., et al., Preparation and in-vitro Evaluation of an Antisense-containing Cationic Liposome against Non-small Cell Lung Cancer: a Comparative Preparation Study. Iran J Pharm Res, 2013. 12(Suppl): p. 3-10.

162. Siddikuzzaman and V.M. Grace, Inhibition of metastatic lung cancer in C57BL/6 mice by liposome encapsulated all trans retinoic acid (ATRA). Int Immunopharmacol, 2012. 14(4): p. 570-9.

163. Lim, W.T., et al., Phase I pharmacokinetic study of a weekly liposomal paclitaxel formulation (Genexol-PM) in patients with solid tumors. Ann Oncol, 2010. 21(2): p. 382-8.

164. Jeong, S.Y., et al., Enhancement of radiotherapeutic effectiveness by temperature-sensitive liposomal 1-methylxanthine. Int J Pharm, 2009. 372(1-2): p. 132-9.

165. Jiang, L., et al., Thiolated chitosan-modified PLA-PCL-TPGS nanoparticles for oral chemotherapy of lung cancer. Nanoscale Res Lett, 2013. 8(1): p. 66.

166. Liu, P., et al., Micro-CT molecular imaging of tumor angiogenesis using a magnetite nano-cluster probe. J Biomed Nanotechnol, 2013. 9(6): p. 1041-9.

167. Karra, N., et al., Safety and proof-of-concept efficacy of inhaled drug loaded nano- and immunonanoparticles in a c-Raf transgenic lung cancer model. Curr Cancer Drug Targets, 2013. 13(1): p. 11-29.

168. Karra, N., et al., Antibody Conjugated PLGA Nanoparticles for Targeted Delivery of Paclitaxel Palmitate: Efficacy and Biofate in a Lung Cancer Mouse Model. Small, 2013. 9(24): p. 42214236.

169. Su, W.P., et al., PLGA nanoparticles codeliver paclitaxel and Stat 3 siRNA to overcome cellular resistance in lung cancer cells. International Journal of Nanomedicine, 2012. 7: p. 4269-4283.

170. Taetz, S., et al., The influence of chitosan content in cationic chitosan/PLGA nanoparticles on the delivery efficiency of antisense 2 '-O-methyl-RNA directed against telomerase in lung cancer cells. European Journal of Pharmaceutics and Biopharmaceutics, 2009. 72(2): p. 358-369. 
171. Atyabi, F., et al., Preparation of pegylated nano-liposomal formulation containing SN-38: In vitro characterization and in vivo biodistribution in mice. Acta Pharmaceutica, 2009. 59(2): p. 133-144.

172. Bakht, M.K., et al., Preparation of radioactive praseodymium oxide as a multifunctional agent in nuclear medicine: expanding the horizons of cancer therapy using nanosized neodymium oxide. Nuclear Medicine Communications, 2013. 34(1): p. 5-12.

173. Torrecilla, D., et al., Anti-tumor efficacy of chitosan-g-poly(ethylene glycol) nanocapsules containing docetaxel: Anti-TMEFF-2 functionalized nanocapsules vs. non-functionalized nanocapsules. European Journal of Pharmaceutics and Biopharmaceutics, 2013. 83(3): p. 330-337.

174. Wang, X.L., R.Z. Xu, and Z.R. Lu, A peptide-targeted delivery system with $\mathrm{pH}$-sensitive amphiphilic cell membrane disruption for efficient receptor-mediated siRNA delivery. Journal of Controlled Release, 2009. 134(3): p. 207-213.

175. Yang, J., et al., A nanoparticle formulation that selectively transfects metastatic tumors in mice. Proceedings of the National Academy of Sciences of the United States of America, 2013. 110(36): p. 14717-14722.

176. Zhao, H., et al., Nanoemulsion loaded with lycobetaine-oleic acid ionic complex: physicochemical characteristics, in vitro, in vivo evaluation, and antitumor activity. International Journal of Nanomedicine, 2013. 8: p. 1959-1973.

177. Das, J., et al., Assessment of drug delivery and anticancer potentials of nanoparticles-loaded siRNA targeting STAT3 in lung cancer, in vitro and in vivo. Toxicol Lett, 2014.

178. Shen, J., et al., Co-delivery of paclitaxel and survivin shRNA by pluronic P85-PEI/TPGS complex nanoparticles to overcome drug resistance in lung cancer. Biomaterials, 2012. 33(33): p. 8613-24.

179. Rakkithawatthana, V., et al., Investigation of gene transferring efficacy through nano-polyplex consisting of methylated N-(4-pyridinylmethyl) chitosan chloride and poly(ethylenimine) in human cell lines. Carbohydrate Polymers, 2010. 80(1): p. 276-284.

180. Ashokan, A., et al., A molecular receptor targeted, hydroxyapatite nanocrystal based multi-modal contrast agent. Biomaterials, 2010. 31(9): p. 2606-2616.

181. Bivas-Benita, M., et al., PLGA-PEI nanoparticles for gene delivery to pulmonary epithelium. European Journal of Pharmaceutics and Biopharmaceutics, 2004. 58(1): p. 1-6.

182. Jiang, L.Q., et al., Thiolated chitosan-modified PLA-PCL-TPGS nanoparticles for oral chemotherapy of lung cancer. Nanoscale Research Letters, 2013. 8: p. 1-11. 
183. Sun, Y.J., et al., Template synthesis of PMAA@ chitosan hollow nanorods for docetaxel delivery. Polymer Chemistry, 2013. 4(8): p. 2489-2495.

184. Salah, R., et al., Anticancer activity of chemically prepared shrimp low molecular weight chitin evaluation with the human monocyte leukaemia cell line, THP-1. International Journal of Biological Macromolecules, 2013. 52: p. 333-339.

185. Potara, M., et al., Chitosan-coated triangular silver nanoparticles as a novel class of biocompatible, highly sensitive plasmonic platforms for intracellular SERS sensing and imaging. Nanoscale, 2013. 5(13): p. 6013-6022.

186. Derakhshandeh, K. and S. Fathi, Role of chitosan nanoparticles in the oral absorption of Gemcitabine. International Journal of Pharmaceutics, 2012. 437(1-2): p. 172-177.

187. Sukumar, U.K., et al., Emerging applications of nanoparticles for lung cancer diagnosis and therapy. International Nano Letters, 2013. 3(1): p. 45.

188. Sun, Q., et al., Enhanced apoptotic effects of dihydroartemisinin-aggregated gelatin and hyaluronan nanoparticles on human lung cancer cells. J Biomed Mater Res B Appl Biomater, 2013.

189. Sun, X.Y. and W.Q. Liang, [Comparison on antitumor activity of cisplatin-loaded liposomes and nanoparticles in vitro]. Zhejiang Da Xue Xue Bao Yi Xue Ban, 2011. 40(4): p. 408-13.

190. Tseng, C.L., et al., The use of biotinylated-EGF-modified gelatin nanoparticle carrier to enhance cisplatin accumulation in cancerous lungs via inhalation. Biomaterials, 2009. 30(20): p. 3476-85.

191. Nguyen, J., et al., Effects of cell-penetrating peptides and pegylation on transfection efficiency of polyethylenimine in mouse lungs. The Journal of Gene Medicine, 2008. 10(11): p. 1236-1246.

192. Koshkina, N.V., et al., Biodistribution and pharmacokinetics of aerosol and intravenously administered DNA-polyethyleneimine complexes: optimization of pulmonary delivery and retention. Mol Ther, 2003. 8(2): p. 249-54.

193. Hong, S.H., et al., Suppression of lung cancer progression by biocompatible glycerol triacrylatespermine-mediated delivery of shAkt1. Int J Nanomedicine, 2012. 7: p. 2293-306.

194. Fadel, M., K. Kassab, and D.A. Fadeel, Zinc phthalocyanine-loaded PLGA biodegradable nanoparticles for photodynamic therapy in tumor-bearing mice. Lasers Med Sci, 2010. 25(2): p. 283-72.

195. Jung, J., et al., Polymeric Nanoparticles Containing Taxanes Enhance Chemoradiotherapeutic Efficacy in Non-small Cell Lung Cancer. International Journal of Radiation Oncology Biology Physics, 2012. 84(1): p. E77-E83. 
196. Beck-Broichsitter, M., et al., Pulmonary drug delivery with aerosolizable nanoparticles in an ex vivo lung model. Int J Pharm, 2009. 367(1-2): p. 169-78.

197. Anselmo, A.C., et al., Delivering Nanoparticles to Lungs while Avoiding Liver and Spleen through Adsorption on Red Blood Cells. Acs Nano, 2013. 7(12): p. 11129-11137.

198. Sadhukha, T., T.S. Wiedmann, and J. Panyam, Inhalable magnetic nanoparticles for targeted hyperthermia in lung cancer therapy. Biomaterials, 2013. 34(21): p. 5163-71.

199. Guthi, J.S., et al., MRI-visible micellar nanomedicine for targeted drug delivery to lung cancer cells. Mol Pharm, 2010. 7(1): p. 32-40.

200. Lu, W., et al., Facile synthesis and characterization of polyethylenimine-coated Fe3O4 superparamagnetic nanoparticles for cancer cell separation. Mol Med Rep, 2014. 9(3): p. 1080-4.

201. Xu, H., et al., Bifunctional magnetic nanoparticles for analysis of aldehyde metabolites in exhaled breath of lung cancer patients. J Chromatogr A, 2014. 1324: p. 29-35.

202. Ghazani, A.A., et al., Molecular characterization of scant lung tumor cells using iron-oxide nanoparticles and micro-nuclear magnetic resonance. Nanomedicine, 2013.

203. Tewes, F., C. Ehrhardt, and A.M. Healy, Superparamagnetic iron oxide nanoparticles (SPIONs)loaded Trojan microparticles for targeted aerosol delivery to the lung. Eur J Pharm Biopharm, 2013.

204. Araya, T., et al., Antitumor effects of inductive hyperthermia using magnetic ferucarbotran nanoparticles on human lung cancer xenografts in nude mice. Onco Targets Ther, 2013. 6: p. 23742.

205. Verma, N.K., et al., Magnetic core-shell nanoparticles for drug delivery by nebulization. J Nanobiotechnology, 2013. 11: p. 1.

206. Conde, J., et al., In vivo tumor targeting via nanoparticle-mediated therapeutic siRNA coupled to inflammatory response in lung cancer mouse models. Biomaterials, 2013. 34(31): p. 7744-7753.

207. Bhat, R., et al., Photo-bio-synthesis of irregular shaped functionalized gold nanoparticles using edible mushroom Pleurotus florida and its anticancer evaluation. Journal of Photochemistry and Photobiology B-Biology, 2013. 125: p. 63-69.

208. Liu, M., et al., Gold nanoparticles trigger apoptosis and necrosis in lung cancer cells with low intracellular glutathione. Journal of Nanoparticle Research, 2013. 15(8). 
209. Choi, Y.J., et al., Cytotoxicity and Genotoxicity Induced by Photothermal Effects of Colloidal Gold Nanorods. Journal of Nanoscience and Nanotechnology, 2013. 13(6): p. 4437-4445.

210. Kao, H.W., et al., Evaluation of EGFR-targeted radioimmuno-gold-nanoparticles as a theranostic agent in a tumor animal model. Bioorganic \& Medicinal Chemistry Letters, 2013. 23(11): p. 3180-3185.

211. Fellahi, O., et al., The antimicrobial effect of silicon nanowires decorated with silver and copper nanoparticles. Nanotechnology, 2013. 24(49).

212. Sankar, R., et al., Origanum vulgare mediated biosynthesis of silver nanoparticles for its antibacterial and anticancer activity. Colloids and Surfaces B-Biointerfaces, 2013. 108: p. 80-84.

213. Gengan, R.M., et al., A549 lung cell line activity of biosynthesized silver nanoparticles using Albizia adianthifolia leaf. Colloids and Surfaces B-Biointerfaces, 2013. 105: p. 87-91.

214. Govender, R., et al., Silver nanoparticles of Albizia adianthifolia: the induction of apoptosis in human lung carcinoma cell line. Journal of Nanobiotechnology, 2013. 11.

215. Vyas, K.M., et al., A new pyrazolone based ternary $\mathrm{Cu}(\mathrm{II})$ complex: Synthesis, characterization, crystal structure, DNA binding, protein binding and anti-cancer activity towards A549 human lung carcinoma cells with a minimum cytotoxicity to non-cancerous cells. Polyhedron, 2013. 65: p. 262-274.

216. Magaye, R., et al., Genotoxicity and carcinogenicity of cobalt-, nickel-and copper-based nanoparticles (Review). Experimental and Therapeutic Medicine, 2012. 4(4): p. 551-561.

217. Thounaojam, M.C., et al., Oxidative stress induced apoptosis of human lung carcinoma (A549) cells by a novel copper nanorod formulation. Food and Chemical Toxicology, 2011. 49(11): p. 29902996.

218. Kankala, S., et al., Pd-N-heterocyclic carbene catalyzed synthesis of piperidine alkene-alkaloids and their anti-cancer evaluation. Bioorg Med Chem Lett, 2014.

219. Zhang, J., et al., Fluorescent metal nanoshell probe to detect single miRNA in lung cancer cell. Anal Chem, 2010. 82(11): p. 4464-71.

220. Wan, R., et al., The role of hypoxia inducible factor-1alpha in the increased MMP-2 and MMP-9 production by human monocytes exposed to nickel nanoparticles. Nanotoxicology, 2011. 5(4): p. $568-82$. 
221. Glista-Baker, E.E., et al., Nickel Nanoparticles Enhance Platelet-Derived Growth Factor-Induced Chemokine Expression by Mesothelial Cells via Prolonged Mitogen-Activated Protein Kinase Activation. American Journal of Respiratory Cell and Molecular Biology, 2012. 47(4): p. 552-561.

222. Wan, R., et al., The role of hypoxia inducible factor-1 alpha in the increased MMP-2 and MMP-9 production by human monocytes exposed to nickel nanoparticles. Nanotoxicology, 2011. 5(4): p. 568-582.

223. Tang, M., P.J. Russell, and A. Khatri, Magnetic nanoparticles: prospects in cancer imaging and therapy. Discov Med, 2007. 7(38): p. 68-74.

224. Wang, M. and N.O. Petersen, Lipid-coated gold nanoparticles promote lamellar body formation in A549 cells. Biochim Biophys Acta, 2013. 1831(6): p. 1089-97.

225. England, C.G., et al., Enhanced penetration into $3 D$ cell culture using two and three layered gold nanoparticles. Int J Nanomedicine, 2013. 8: p. 3603-3617.

226. Peng, G., et al., Diagnosing lung cancer in exhaled breath using gold nanoparticles. Nat Nano, 2009. 4(10): p. 669-673.

227. Barash, O., et al., Classification of lung cancer histology by gold nanoparticle sensors. Nanomedicine, 2012. 8(5): p. 580-9.

228. Bhat, R., et al., Photo-bio-synthesis of irregular shaped functionalized gold nanoparticles using edible mushroom Pleurotus florida and its anticancer evaluation. Journal of Photochemistry and Photobiology B: Biology, 2013. 125(0): p. 63-69.

229. Ahamed, M., Toxic response of nickel nanoparticles in human lung epithelial A549 cells. Toxicology in Vitro, 2011. 25(4): p. 930-936.

230. Cheng, F.Y., C.T. Chen, and C.S. Yeh, Comparative efficiencies of photothermal destruction of malignant cells using antibody-coated silica@Au nanoshells, hollow Au/Ag nanospheres and Au nanorods. Nanotechnology, 2009. 20(42).

231. Liu, H.Y., et al., Photothermal therapy of Lewis lung carcinoma in mice using gold nanoshells on carboxylated polystyrene spheres. Nanotechnology, 2008. 19(45).

232. Stober, W., A. Fink, and E. Bohn, Controlled Growth of Monodisperse Silica Spheres in Micron Size Range. Journal of Colloid and Interface Science, 1968. 21(1).

233. Oldenburg, S.J., et al., Nanoengineering of optical resonances. Chemical Physics Letters, 1998. 288(2-4): p. 243-247. 
234. Cheng, F.Y., C.T. Chen, and C.S. Yeh, Comparative efficiencies of photothermal destruction of malignant cells using antibody-coated silica@Au nanoshells, hollow Au/Ag nanospheres and Au nanorods. Nanotechnology, 2009. 20(42): p. 425104.

235. Bagley, A.F., et al., Plasmonic Photothermal Heating of Intraperitoneal Tumors through the Use of an Implanted Near-Infrared Source. ACS Nano, 2013. 7(9): p. 8089-8097.

236. $\mathrm{Wu}, \mathrm{P}$., et al., High specific detection and near-infrared photothermal therapy of lung cancer cells with high SERS active aptamer-silver-gold shell-core nanostructures. Analyst, 2013. 138(21): p. 6501-10.

237. Cobley, C.M., et al., Targeting gold nanocages to cancer cells for photothermal destruction and drug delivery. Expert Opinion on Drug Delivery, 2010. 7(5): p. 577-587.

238. Huang, H.C., et al., Inorganic nanoparticles for cancer imaging and therapy. J Control Release, 2011. 155(3): p. 344-57.

239. Stanwix, H., First clinical trial of gold nanoshells for treating lung cancer to be carried out in the USA. Nanomedicine, 2013. 8(1): p. 10-10.

240. Ashokan, A., et al., A molecular receptor targeted, hydroxyapatite nanocrystal based multi-modal contrast agent. Biomaterials, 2010. 31(9): p. 2606-16.

241. Lin, Y.C., et al., Mesoporous silica nanoparticles synthesized from liquid crystal display manufacturing extracts as a potential candidate for a drug delivery carrier: evaluation of their safety and biocompatibility. Int J Nanomedicine, 2013. 8: p. 3833-42.

242. Shen, J., et al., The use of hollow mesoporous silica nanospheres to encapsulate bortezomib and improve efficacy for non-small cell lung cancer therapy. Biomaterials, 2014. 35(1): p. 316-26.

243. Huang, S., et al., Luminescent GdVO4:Eu3+ functionalized mesoporous silica nanoparticles for magnetic resonance imaging and drug delivery. Dalton Trans, 2013. 42(18): p. 6523-30.

244. Ahamed, M., Silica nanoparticles-induced cytotoxicity, oxidative stress and apoptosis in cultured A431 and A549 cells. Hum Exp Toxicol, 2013. 32(2): p. 186-95.

245. Foa, V. and S. Basilico, [Chemical and physical characteristics and toxicology of man-made mineral fibers]. Med Lav, 1999. 90(1): p. 10-52.

246. Silva, R.M., et al., Biological Response to Nano-Scale Titanium Dioxide (Tio2): Role of Particle Dose, Shape, and Retention. Journal of Toxicology and Environmental Health-Part a-Current Issues, 2013. 76(16): p. 953-972. 
247. Jugan, M.L., et al., Cytotoxic and genotoxic impact of TiO2 nanoparticles on A549 cells. J Biomed Nanotechnol, 2011. 7(1): p. 22-3.

248. Chusuei, C.C., et al., Cytotoxicity in the age of nano: The role of fourth period transition metal oxide nanoparticle physicochemical properties. Chemico-Biological Interactions, 2013. 206(2): p. 319326.

249. Wang, D.G., et al., Zinc oxide nanoparticles inhibit Ca2+-ATPase expression in human lens epithelial cells under UVB irradiation. Toxicology in Vitro, 2013. 27(8): p. 2117-2126.

250. Min, L.F., et al., Magnetic iron oxide nanoparticles carrying PTEN gene to reverse cisplatinresistance of A549/CDDP cell lines. Journal of Central South University of Technology, 2012. 19(2): p. 331-339.

251. Antonicelli, A., et al., EGFR-targeted therapy for non-small cell lung cancer: focus on EGFR oncogenic mutation. Int J Med Sci, 2013. 10(3): p. 320-30.

252. Lurje, G. and H.J. Lenz, EGFR Signaling and Drug Discovery. Oncology, 2009. 77(6): p. 400-410.

253. Jensen, A.D., et al., Treatment of non-small cell lung cancer with intensity-modulated radiation therapy in combination with cetuximab: the NEAR protocol (NCT00115518). Bmc Cancer, 2006. 6.

254. Sundarraj, S., EGFR antibody conjugated mesoporous silica nanoparticles for cytosolic phospholipase A2a targeted nonsmall lung cancer therapy. Journal of Cell Science and Therapy, 2012. 3(7).

255. Yuan, H., et al., Preparation and evaluation of SiO2-deposited stearic acid-g-chitosan nanoparticles for doxorubicin delivery. Int J Nanomedicine, 2012. 7: p. 5119-28.

256. Srivastava, R.K., et al., Nano-titanium dioxide induces genotoxicity and apoptosis in human lung cancer cell line, A549. Hum Exp Toxicol, 2013. 32(2): p. 153-66.

257. Srivastava, R.K., et al., Ameliorative effects of dimetylthiourea and $\mathrm{N}$-acetylcysteine on nanoparticles induced cyto-genotoxicity in human lung cancer cells-A549. PLoS One, 2011. 6(9): p. e25767.

258. Al-Hallak, M.H., et al., Pulmonary toxicity of polysorbate-80-coated inhalable nanoparticles; in vitro and in vivo evaluation. AAPS J, 2010. 12(3): p. 294-9.

259. Oberdorster, G., et al., Principles for characterizing the potential human health effects from exposure to nanomaterials: elements of a screening strategy. Part Fibre Toxicol, 2005. 2: p. 8. 
260. Ahmed, M. and N.K. Cheung, Engineering anti-GD2 monoclonal antibodies for cancer immunotherapy. FEBS Lett, 2014. 588(2): p. 288-97.

261. Caron, J., et al., Improving the antitumor activity of squalenoyl-paclitaxel conjugate nanoassemblies by manipulating the linker between paclitaxel and squalene. Adv Healthc Mater, 2013. 2(1): p. $172-85$.

262. Guan, Y.Y., et al., Selective eradication of tumor vascular pericytes by peptide-conjugated nanoparticles for antiangiogenic therapy of melanoma lung metastasis. Biomaterials, 2014.

263. Razzak, R., et al., The biodistribution and pharmacokinetic evaluation of choline-bound gold nanoparticles in a human prostate tumor xenograft model. Clin Invest Med, 2013. 36(3): p. E13342.

264. Wang, H., et al., Folic acid-modified dendrimer-entrapped gold nanoparticles as nanoprobes for targeted CT imaging of human lung adencarcinoma. Biomaterials, 2013. 34(2): p. 470-80.

265. Wehrung, D., et al., Antitumor efficacy and tolerability of systemically administered gallium acetylacetonate-loaded gelucire-stabilized nanoparticles. J Biomed Nanotechnol, 2013. 9(6): p. 1029-40.

266. Zhang, Y., W.Y. Kim, and L. Huang, Systemic delivery of gemcitabine triphosphate via LCP nanoparticles for NSCLC and pancreatic cancer therapy. Biomaterials, 2013. 34(13): p. 3447-58.

267. Park, S., et al., Cellular Toxicity of Various Inhalable Metal Nanoparticles on Human Alveolar Epithelial Cells. Inhalation Toxicology, 2007. 19(s1): p. 59-65.

268. Karlsson, H.L., et al., Copper Oxide Nanoparticles Are Highly Toxic: A Comparison between Metal Oxide Nanoparticles and Carbon Nanotubes. Chemical Research in Toxicology, 2008. 21(9): p. 1726-1732.

269. Londahl, J., et al., Experimental determination of the respiratory tract deposition of diesel combustion particles in patients with chronic obstructive pulmonary disease. Particle and Fibre Toxicology, 2012. 9.

270. Anderson, P.J., J.D. Wilson, and F.C. Hiller, Respiratory-Tract Deposition of Ultrafine Particles in Subjects with Obstructive or Restrictive Lung-Disease. Chest, 1990. 97(5): p. 1115-1120.

271. Daigle, C.C., et al., Ultrafine particle deposition in humans during rest and exercise. Inhalation Toxicology, 2003. 15(6): p. 539-552. 
272. Moller, W., et al., Deposition, retention, and translocation of ultrafine particles from the central airways and lung periphery. American Journal of Respiratory and Critical Care Medicine, 2008. 177(4): p. 426-432.

273. Mitchell, L.A., et al., Pulmonary and Systemic Immune Response to Inhaled Multiwalled Carbon Nanotubes. Toxicological Sciences, 2007. 100(1): p. 203-214.

274. Bondarenko, O., et al., Toxicity of $\mathrm{Ag}, \mathrm{CuO}$ and $\mathrm{ZnO}$ nanoparticles to selected environmentally relevant test organisms and mammalian cells in vitro: a critical review. Archives of Toxicology, 2013. 87(7): p. 1181-1200.

275. Yu, L.E., et al., Translocation and effects of gold nanoparticles after inhalation exposure in rats. Nanotoxicology, 2007. 1(3): p. 235-242.

276. De Jong, W.H., et al., Particle size-dependent organ distribution of gold nanoparticles after intravenous administration. Biomaterials, 2008. 29(12): p. 1912-1919.

277. Chen, Y.S., et al., Assessment of the In Vivo Toxicity of Gold Nanoparticles. Nanoscale Research Letters, 2009. 4(8): p. 858-864.

278. Ferin, J., G. Oberdorster, and D.P. Penney, Pulmonary Retention of Ultrafine and Fine Particles in Rats. American Journal of Respiratory Cell and Molecular Biology, 1992. 6(5): p. 535-542.

279. Geys, J., et al., Acute Toxicity and Prothrombotic Effects of Quantum Dots: Impact of Surface Charge. Environmental Health Perspectives, 2008. 116(12): p. 1607-1613.

280. Sayes, C.M., et al., Comparative pulmonary toxicity assessments of C60 water suspensions in rats: few differences in fullerene toxicity in vivo in contrast to in vitro profiles. Nano Lett, 2007. 7(8): p. 2399-406.

281. Sayes, C.M., K.L. Reed, and D.B. Warheit, Assessing toxicity of fine and nanoparticles: comparing in vitro measurements to in vivo pulmonary toxicity profiles. Toxicol Sci, 2007. 97(1): p. 163-80.

282. Kong, G., R.D. Braun, and M.W. Dewhirst, Hyperthermia enables tumor-specific nanoparticle delivery: effect of particle size. Cancer Res, 2000. 60(16): p. 4440-5.

283. Libutti, S.K., et al., Phase I and pharmacokinetic studies of CYT-6091, a novel PEGylated colloidal gold-rhTNF nanomedicine. Clin Cancer Res, 2010. 16(24): p. 6139-49.

284. Murphy, C.J., et al., Gold nanoparticles in biology: beyond toxicity to cellular imaging. Acc Chem Res, 2008. 41(12): p. 1721-30. 
285. Zamboni, W.C., et al., Best practices in cancer nanotechnology: perspective from NCI nanotechnology alliance. Clin Cancer Res, 2012. 18(12): p. 3229-41.

286. Alkilany, A.M. and C.J. Murphy, Toxicity and cellular uptake of gold nanoparticles: what we have learned so far? Journal of Nanoparticle Research, 2010. 12(7): p. 2313-2333.

287. Kennedy, L.C., et al., A New Era for Cancer Treatment: Gold-Nanoparticle-Mediated Thermal Therapies. Small, 2011. 7(2): p. 169-183.

288. Goodman, C.M., et al., Toxicity of gold nanoparticles functionalized with cationic and anionic side chains. Bioconjugate Chemistry, 2004. 15(4): p. 897-900.

289. Chabner, B.A., Cancer Chemotherapy and Biotherapy: Principles and Practice. 5 ed. 2011, Philadelphia, PA: Lippincott Williams \& Wilkins.

290. Vinci, M., et al., Advances in establishment and analysis of three-dimensional tumor spheroid-based functional assays for target validation and drug evaluation. Bmc Biology, 2012. 10.

291. Krock, B.L., N. Skuli, and M.C. Simon, Hypoxia-induced angiogenesis: good and evil. Genes Cancer, 2011. 2(12): p. 1117-33.

292. van de Ven, A.L., et al., Integrated intravital microscopy and mathematical modeling to optimize nanotherapeutics delivery to tumors. AIP Adv, 2012. 2(1): p. 11208.

293. Brigger, I., C. Dubernet, and P. Couvreur, Nanoparticles in cancer therapy and diagnosis. Advanced Drug Delivery Reviews, 2002. 54(5): p. 631-651.

294. Jain, R.K., Transport of molecules in the tumor interstitium: a review. Cancer Res, 1987. 47(12): p. 3039-51.

295. van de Ven, A.L., et al., Modeling of nanotherapeutics delivery based on tumor perfusion. New Journal of Physics, 2013. 15.

296. Wu, M., et al., The effect of interstitial pressure on tumor growth: Coupling with the blood and lymphatic vascular systems. J Theor Biol, 2013. 320: p. 131-51.

297. Michor, F., et al., What does physics have to do with cancer? Nature Reviews Cancer, 2011. 11(9): p. 657-670.

298. Wong, C., et al., Multistage nanoparticle delivery system for deep penetration into tumor tissue. Proceedings of the National Academy of Sciences of the United States of America, 2011. 108(6): p. 2426-2431. 
299. Goodman, T.T., P.L. Olive, and S.H. Pun, Increased nanoparticle penetration in collagenase-treated multicellular spheroids. Int J Nanomedicine, 2007. 2(2): p. 265-74.

300. Cabral, H., et al., Accumulation of sub-100 nm polymeric micelles in poorly permeable tumours depends on size. Nat Nanotechnol, 2011. 6(12): p. 815-23.

301. Garrett, N., M. Whiteman, and J. Moger, Imaging the uptake of gold nanoshells in live cells using plasmon resonance enhanced four wave mixing microscopy. Optics Express, 2011. 19(18): p. 17563-17574.

302. Liang, Z.S., et al., Surface-modified gold nanoshells for enhanced cellular uptake. Journal of Biomedical Materials Research Part A, 2011. 98A(4): p. 479-487.

303. Chithrani, B.D., A.A. Ghazani, and W.C.W. Chan, Determining the size and shape dependence of gold nanoparticle uptake into mammalian cells. Nano Letters, 2006. 6(4): p. 662-668.

304. Frieboes, H.B., et al., Prediction of drug response in breast cancer using integrative experimental/computational modeling. Cancer Res, 2009. 69(10): p. 4484-92.

305. Srinivas, A., et al., Acute inhalation toxicity of cerium oxide nanoparticles in rats. Toxicology Letters, 2011. 205(2): p. 105-115.

306. Frens, G., Controlled Nucleation for the Regulation of the Particle Size in Monodisperse Gold Solutions. Nature Physical Sciences, 1973. 241: p. 20-22.

307. Kalele, S., et al., Nanoshell particles: synthesis, properties and applications. Current Science, 2006. 91(8): p. 1038-1052.

308. Preston, T.C. and R. Signorell, Growth and Optical Properties of Gold Nanoshells Prior to the Formation of a Continuous Metallic Layer. Acs Nano, 2009. 3(11): p. 3696-3706.

309. Duff, D.G., A. Baiker, and P.P. Edwards, A New Hydrosol of Gold Clusters. Journal of the Chemical Society-Chemical Communications, 1993(1): p. 96-98.

310. Guan, M., et al., N-trimethyl chitosan nanoparticle-encapsulated lactosyl-norcantharidin for liver cancer therapy with high targeting efficacy. Nanomedicine-Nanotechnology Biology and Medicine, 2012. 8(7): p. 1172-1181.

311. Wang, J., et al., Size- and pathotropism-driven targeting and washout-resistant effects of boronic acid-rich protein nanoparticles for liver cancer regression. Journal of Controlled Release, 2013. 168(1): p. 1-9. 
312. Lai, L.F. and H.X. Guo, Preparation of new 5-fluorouracil-loaded zein nanoparticles for liver targeting. International Journal of Pharmaceutics, 2011. 404(1-2): p. 317-323.

313. Kimling, J., et al., Turkevich method for gold nanoparticle synthesis revisited. J Phys Chem B, 2006. 110(32): p. 15700-7.

314. Leung, K., Gold-polyethylene glycol nanoshells, in Molecular Imaging and Contrast Agent Database (MICAD). 2004: Bethesda (MD).

315. Tullman, J.A., et al., Tunable assembly of peptide-coated gold nanoparticles. Plasmonics, 2007. 2(3): p. 119-127.

316. Gobin, A.M., et al., Near-infrared resonant nanoshells for combined optical imaging and photothermal cancer therapy. Nano Lett, 2007. 7(7): p. 1929-34.

317. O'Neal, D.P., et al., Photo-thermal tumor ablation in mice using near infrared-absorbing nanoparticles. Cancer Lett, 2004. 209(2): p. 171-6.

318. Jokerst, J.V., et al., Nanoparticle PEGylation for imaging and therapy. Nanomedicine (Lond), 2011. 6(4): p. 715-28.

319. Maldiney, T., et al., Effect of core diameter, surface coating, and PEG chain length on the biodistribution of persistent luminescence nanoparticles in mice. ACS Nano, 2011. 5(2): p. 85462.

320. Gu, X., et al., Preparation and characterization of a lovastatin-loaded protein-free nanostructured lipid carrier resembling high-density lipoprotein and evaluation of its targeting to foam cells. AAPS PharmSciTech, 2011. 12(4): p. 1200-8.

321. Wen, S., et al., Surface modification and PEGylation of branched polyethyleneimine for improved biocompatibility. Journal of Applied Polymer Science, 2013. 128(6): p. 3807-3813.

322. Lewis, B.A. and D.M. Engelman, Lipid bilayer thickness varies linearly with acyl chain length in fluid phosphatidylcholine vesicles. J Mol Biol, 1983. 166(2): p. 211-7.

323. Nagle, J.F. and S. Tristram-Nagle, Structure of lipid bilayers. Biochim Biophys Acta, 2000. 1469(3): p. 159-95.

324. El Harchaoui, K., et al., High-density lipoprotein particle size and concentration and coronary risk. Ann Intern Med, 2009. 150(2): p. 84-93. 
325. Yen, J.C., F.J. Chang, and S. Chang, A new criterion for automatic multilevel thresholding. IEEE Trans Image Process, 1995. 4(3): p. 370-8.

326. Takahashi, H., et al., Modification of gold nanorods using phosphatidylcholine to reduce cytotoxicity. Langmuir, 2006. 22(1): p. 2-5.

327. Lowery, A.R., et al., Immunonanoshells for targeted photothermal ablation of tumor cells. International Journal of Nanomedicine, 2006. 1(2): p. 149-154.

328. Kojima, C., Y. Hirano, and K. Kono, Chapter 7 - Preparation of complexes of liposomes with gold nanoparticles. Methods Enzymol, 2009. 464: p. 131-45.

329. Jiang, J., P. Nilsson-Ehle, and N. Xu, Influence of liver cancer on lipid and lipoprotein metabolism. Lipids Health Dis, 2006. 5: p. 4.

330. Shahzad, M.M.K., et al., Targeted Delivery of Small Interfering RNA Using Reconstituted HighDensity Lipoprotein Nanoparticles. Neoplasia, 2011. 13(4): p. 309-U142.

331. Ng, K.K., J.F. Lovell, and G. Zheng, Lipoprotein-inspired nanoparticles for cancer theranostics. Acc Chem Res, 2011. 44(10): p. 1105-13.

332. Blume, G. and G. Cevc, Molecular mechanism of the lipid vesicle longevity in vivo. Biochim Biophys Acta, 1993. 1146(2): p. 157-68.

333. Krilov, D., et al., FT-IR spectroscopy of lipoproteins-A comparative study. Spectrochimica Acta Part a-Molecular and Biomolecular Spectroscopy, 2009. 73(4): p. 701-706.

334. Semalty, A., et al., Preparation and characterization of phospholipid complexes of naringenin for effective drug delivery. Journal of Inclusion Phenomena and Macrocyclic Chemistry, 2010. 67(34): p. 253-260.

335. Gupta, A.K. and A.S. Curtis, Surface modified superparamagnetic nanoparticles for drug delivery: interaction studies with human fibroblasts in culture. J Mater Sci Mater Med, 2004. 15(4): p. 4936.

336. Frieboes, H.B., et al., A computational model for predicting nanoparticle accumulation in tumor vasculature. PLoS One, 2013. 8(2): p. e56876.

337. van de Ven, A.L., et al., Modeling of nanotherapeutics delivery based on tumor perfusion. New $\mathbf{J}$ Phys, 2013. 15: p. 55004. 
338. Wang, J., et al., Improving delivery and efficacy of nanomedicines in solid tumors: role of tumor priming. Nanomedicine, 2011. 6(9): p. 1605-1620.

339. Li, S.D. and L. Huang, Nanoparticles evading the reticuloendothelial system: Role of the supported bilayer. Biochimica Et Biophysica Acta-Biomembranes, 2009. 1788(10): p. 2259-2266.

340. De Jong, W.H. and P.J.A. Borm, Drug delivery and nanoparticles: Applications and hazards. International Journal of Nanomedicine, 2008. 3(2): p. 133-149.

341. Zhang, Z., et al., HDL-mimicking peptide-lipid nanoparticles with improved tumor targeting. Small, 2010. 6(3): p. 430-7.

342. Jain, R.K. and T. Stylianopoulos, Delivering nanomedicine to solid tumors. Nat Rev Clin Oncol, 2010. 7(11): p. 653-64.

343. Cairns, R.A., I.S. Harris, and T.W. Mak, Regulation of cancer cell metabolism. Nature Reviews Cancer, 2011. 11(2): p. 85-95.

344. Raghunand, N., R.A. Gatenby, and R.J. Gillies, Microenvironmental and cellular consequences of altered blood flow in tumours. British Journal of Radiology, 2003. 76: p. S11-S22.

345. Baxter, L.T. and R.K. Jain, Transport of fluid and macromolecules in tumors. I. Role of interstitial pressure and convection. Microvasc Res, 1989. 37(1): p. 77-104.

346. Baxter, L.T. and R.K. Jain, Transport of fluid and macromolecules in tumors. II. Role of heterogeneous perfusion and lymphatics. Microvasc Res, 1990. 40(2): p. 246-63.

347. Huo, S., et al., Superior Penetration and Retention Behavior of $50 \mathrm{~nm}$ Gold Nanoparticles in Tumors. Cancer Res, 2013. 73(1): p. 319-30.

348. Sadauskas, E., et al., Kupffer cells are central in the removal of nanoparticles from the organism. Part Fibre Toxicol, 2007. 4: p. 10.

349. Patra, C.R., et al., Targeted delivery of gemcitabine to pancreatic adenocarcinoma using cetuximab as a targeting agent. Cancer Research, 2008. 68(6): p. 1970-1978.

350. Zheng, J., et al., SEM X-ray microanalysis of nanoparticles present in tissue or cultured cell thin sections. Methods Mol Biol, 2011. 697: p. 93-9.

351. Zarogoulidis, P., et al., Inhaled cisplatin deposition and distribution in lymph nodes in stage II lung cancer patients. Future Oncology, 2013. 9(9): p. 1307-1313. 
352. Jeon, S.O., et al., Enhanced percutaneous delivery of recombinant human epidermal growth factor employing nano-liposome system. Journal of Microencapsulation, 2012. 29(3): p. 234-241.

353. Darwiche, K., et al., "One-stop shop" spectral imaging for rapid on-site diagnosis of lung cancer: a future concept in nano-oncology. International Journal of Nanomedicine, 2013. 8: p. 4533-4542.

354. Luo, J.F., et al., The Incidence and Survival Rate of Population-Based Pancreatic Cancer Patients: Shanghai Cancer Registry 2004-2009. Plos One, 2013. 8(10).

355. Vogel, W., D.G. Duff, and A. Baiker, X-Ray Structure of a New Hydrosol of Gold Clusters. Langmuir, 1995. 11(2): p. 401-404.

356. Pisal, D.S. and S.V. Balu-Iyer, Phospholipid binding improves plasma survival of factor VIII. Thromb Haemost, 2010. 104(5): p. 1073-5.

357. Hironobu Takahashi, Y.N., Takuro Niidome,Kenji Kaneko,Hideya Kawasaki, and Sunao Yamada, Modification of Gold Nanorods Using Phosphatidylcholine to Reduce Cytotoxicity. Langmuir, 2006. 22: p. 2-5.

358. Zhang, L., et al., Nanoparticles in medicine: Therapeutic applications and developments. Clinical Pharmacology \& Therapeutics, 2008. 83(5): p. 761-769.

359. Schellekens, H., W.E. Hennink, and V. Brinks, The Immunogenicity of Polyethylene Glycol: Facts and Fiction. Pharmaceutical Research, 2013. 30(7): p. 1729-1734.

360. Green, M., et al., Abraxane, a novel cremophor-free, albumin-bound particle form of paclitaxel for the treatment of advanced non-small-cell lung cancer. Ann. Oncol., 2006. 17(8): p. 1263 - 1268.

361. Guo, C.S., et al., Photothermal ablation cancer therapy using homogeneous CsxWO3 nanorods with broad near-infrared absorption. Nanoscale, 2013. 5(14): p. 6469-6478.

362. Li, Y., et al., Copper sulfide nanoparticles for photothermal ablation of tumor cells. Nanomedicine (Lond), 2010. 5(8): p. 1161-71.

363. Grosges, T., et al., Nanoshells for photothermal therapy: a Monte-Carlo based numerical study of their design tolerance. Biomedical Optics Express, 2011. 2(6): p. 1584-1596.

364. Guo, Y., et al., Photothermal ablation of pancreatic cancer cells with hybrid iron-oxide core goldshell nanoparticles. International Journal of Nanomedicine, 2013. 8: p. 3437-3446. 
365. Kempen, P.J., et al., A scanning transmission electron microscopy approach to analyzing large volumes of tissue to detect nanoparticles. Microsc Microanal, 2013. 19(5): p. 1290-7.

366. Costa, P.M., et al., Tumor-targeted Chlorotoxin-coupled Nanoparticles for Nucleic Acid Delivery to Glioblastoma Cells: A Promising System for Glioblastoma Treatment. Mol Ther Nucleic Acids, 2013. 2: p. e100.

367. Weinkauf, H. and B.F. Brehm-Stecher, Enhanced dark field microscopy for rapid artifact-free detection of nanoparticle binding to Candida albicans cells and hyphae. Biotechnol J, 2009. 4(6): p. 871-9.

368. Mayrovitz, H.N., Skin capillary metrics and hemodynamics in the hairless mouse. Microvasc Res, 1992. 43(1): p. 46-59.

369. Heindryckx, F., et al., Kinetics of angiogenic changes in a new mouse model for hepatocellular carcinoma. Mol Cancer, 2010. 9: p. 219.

370. Wu, M., et al., The effect of interstitial pressure on therapeutic agent transport: Coupling with the tumor blood and lymphatic vascular systems. J Theor Biol, 2014.

371. Frieboes, H., et al., Nanotechnology in Cancer Drug Therapy: A Biocomputational Approach, in BioMEMS and Biomedical Nanotechnology, M. Ferrari, A. Lee, and L.J. Lee, Editors. 2006, Springer US. p. 435-460.

372. Jain, S., D.G. Hirst, and J.M. O'Sullivan, Gold nanoparticles as novel agents for cancer therapy. British Journal of Radiology, 2012. 85(1010): p. 101-113.

373. Clegg, A., et al., Clinical and cost effectiveness of paclitaxel, docetaxel, gemcitabine, and vinorelbine in non-small cell lung cancer: a systematic review. Thorax, 2002. 57(1): p. 20-8.

374. Ohndorf, U.-M., et al., Basis for recognition of cisplatin-modified DNA by high-mobility-group proteins. Nature, 1999. 399(6737): p. 708-712.

375. Horwitz, S.B., Taxol (paclitaxel): mechanisms of action. Ann Oncol, 1994. 5 Suppl 6: p. S3-6.

376. Rowinsky, E.K., et al., Clinical toxicities encountered with paclitaxel (Taxol). Semin Oncol, 1993. 20(4 Suppl 3): p. 1-15.

377. Gelderblom, H., et al., Cremophor EL: the drawbacks and advantages of vehicle selection for drug formulation. European Journal of Cancer, 2001. 37(13): p. 1590-1598. 
378. Madan, J., et al., Long-circulating poly(ethylene glycol)-grafted gelatin nanoparticles customized for intracellular delivery of noscapine: preparation, in-vitro characterization, structure elucidation, pharmacokinetics, and cytotoxicity analyses. Anti-Cancer Drugs, 2011. 22(6): p. 543-555.

379. Chen, H., et al., Characterization of pH-and Temperature-sensitive Hydrogel Nanoparticles for Controlled Drug Release. PDA Journal of Pharmaceutical Science and Technology, 2007. 61(4): p. 303-313.

380. Nichols, J.W. and Y.H. Bae, Odyssey of a cancer nanoparticle: from injection site to site of action. Nano Today, 2012. 7(6): p. 606-618.

381. Longmire, M., P.L. Choyke, and H. Kobayashi, Clearance properties of nano-sized particles and molecules as imaging agents: considerations and caveats. Nanomedicine, 2008. 3(5): p. 703-717.

382. Hwu, J.R., et al., Targeted Paclitaxel by Conjugation to Iron Oxide and Gold Nanoparticles. Journal of the American Chemical Society, 2009. 131(1): p. 66-+.

383. Ding, Y., et al., The performance of thiol-terminated PEG-paclitaxel-conjugated gold nanoparticles. Biomaterials, 2013. 34(38): p. 10217-10227.

384. Shi, Y., J. Goodisman, and J.C. Dabrowiak, Cyclodextrin Capped Gold Nanoparticles as a Delivery Vehicle for a Prodrug of Cisplatin. Inorganic Chemistry, 2013. 52(16): p. 9418-9426.

385. Sanchez-Paradinas, S., et al., Enhanced cytotoxic activity of bile acid cisplatin derivatives by conjugation with gold nanoparticles. J Inorg Biochem, 2014. 131: p. 8-11.

386. Comenge, J., et al., Detoxifying antitumoral drugs via nanoconjugation: the case of gold nanoparticles and cisplatin. PLoS One, 2012. 7(10): p. e47562.

387. Craig, G.E., et al., Cisplatin-Tethered Gold Nanoparticles That Exhibit Enhanced Reproducibility, Drug Loading, and Stability: a Step Closer to Pharmaceutical Approval? Inorganic Chemistry, 2012. 51(6): p. 3490-3497.

388. Comenge, J., et al., Exploring the binding of Pt drugs to gold nanoparticles for controlled passive release of cisplatin. Journal of Controlled Release, 2010. 148(1): p. E31-E32.

389. Panczyk, T., et al., Molecular Dynamics Study of Cisplatin Release from Carbon Nanotubes Capped by Magnetic Nanoparticles. Journal of Physical Chemistry C, 2013. 117(33): p. 17327-17336.

390. Likhitkar, S. and A.K. Bajpai, Magnetically controlled release of cisplatin from superparamagnetic starch nanoparticles. Carbohydrate Polymers, 2012. 87(1): p. 300-308. 
391. Barzegar-Jalali, M., et al., Kinetic analysis of drug release from nanoparticles. Journal of Pharmacy and Pharmaceutical Sciences, 2008. 11(1): p. 167-177.

392. Fonseca, C., S. Simoes, and R. Gaspar, Paclitaxel-loaded PLGA nanoparticles: preparation, physicochemical characterization and in vitro anti-tumoral activity. Journal of Controlled Release, 2002. 83(2): p. 273-286.

393. Dash, S., et al., Kinetic Modeling on Drug Release from Controlled Drug Delivery Systems. Acta Poloniae Pharmaceutica, 2010. 67(3): p. 217-223.

394. Arifin, D.Y., L.Y. Lee, and C.H. Wang, Mathematical modeling and simulation of drug release from microspheres: Implications to drug delivery systems. Advanced Drug Delivery Reviews, 2006. 58(12-13): p. 1274-1325.

395. Collins, R., Mathematical modelling of controlled release from implanted drug-impregnated monoliths. Pharmaceutical Science \& Technology Today, 1998. 1(6): p. 269-276.

396. Bettini, R., et al., Translocation of drug particles in HPMC matrix gel layer: effect of drug solubility and influence on release rate. Journal of Controlled Release, 2001. 70(3): p. 383-391.

397. Suleymanoglu, E., The use of infrared spectroscopy for following drug-membrane interactions: Probing paclitaxel (Taxol)-cell phospholipid surface recognition. Electronic Journal of Biomedicine, 2009. 3: p. 19-35.

398. Siepmann, J. and N.A. Peppas, Modeling of drug release from delivery systems based on hydroxypropyl methylcellulose (HPMC). Advanced Drug Delivery Reviews, 2012. 64, Supplement(0): p. 163-174.

399. Hall, J.B., et al., Characterization of nanoparticles for therapeutics. Nanomedicine (Lond), 2007. 2(6): p. 789-803.

400. Benkovicova, M., et al., Preparation of gold nanoparticles for plasmonic applications. Thin Solid Films, 2013. 543: p. 138-141.

401. Bootz, A., et al., Comparison of scanning electron microscopy, dynamic light scattering and analytical ultracentrifugation for the sizing of poly(butyl cyanoacrylate) nanoparticles. Eur $\mathrm{J}$ Pharm Biopharm, 2004. 57(2): p. 369-75.

402. Gandola, Y.B., et al., Mitogenic Effects of Phosphatidylcholine Nanoparticles on MCF-7 Breast Cancer Cells. Biomed Research International, 2014.

403. Blume, G. and G. Cevc, Molecular Mechanism of the Lipid Vesicle Longevity Invivo. Biochimica Et Biophysica Acta, 1993. 1146(2): p. 157-168. 
404. Xiao, K., et al., The effect of surface charge on in vivo biodistribution of PEG-oligocholic acid based micellar nanoparticles. Biomaterials, 2011. 32(13): p. 3435-3446.

405. Frohlich, E., The role of surface charge in cellular uptake and cytotoxicity of medical nanoparticles. International Journal of Nanomedicine, 2012. 7: p. 5577-5591.

406. Yah, C.S., The toxicity of Gold Nanoparticles in relation to their physiochemical properties. Biomedical Research-India, 2013. 24(3): p. 400-413.

407. Wang, F., et al., FT-IR study of the interaction between phosphatidylcholine and bovine serum albumin. Acta Physico-Chimica Sinica, 2004. 20(10): p. 1186-1190.

408. Li, Y., S. Lim, and C.P. Ooi, Fabrication of Cisplatin-Loaded Poly(lactide-co-glycolide) Composite Microspheres for Osteosarcoma Treatment. Pharmaceutical Research, 2012. 29(3): p. 756-769.

409. Scherphof, G., et al., Disintegration of Phosphatidylcholine Liposomes in Plasma as a Result of Interaction with High-Density Lipoproteins. Biochimica Et Biophysica Acta, 1978. 542(2): p. 296307.

410. Costa, P., J. Manuel, and S. Lobo, Modeling and comparison of dissolution profiles. European Journal of Pharmaceutical Sciences, 2001. 13(2): p. 123-133.

411. Dash, S., et al., Kinetic modeling on drug release from controlled drug delivery systems. Acta Pol Pharm, 2010. 67(3): p. 217-23.

412. El-Sayed, I.H., X.H. Huang, and M.A. El-Sayed, Selective laser photo-thermal therapy of epithelial carcinoma using anti-EGFR antibody conjugated gold nanoparticles. Cancer Letters, 2006. 239(1): p. 129-135.

413. van de Ven, A.L., et al., Integrated intravital microscopy and mathematical modeling to optimize nanotherapeutics delivery to tumors. AIP Advances, 2012. 2(1): p. - .

414. Eklund, L., M. Bry, and K. Alitalo, Mouse models for studying angiogenesis and lymphangiogenesis in cancer. Molecular Oncology, 2013. 7(2): p. 259-282.

415. Warren, K.E., Novel therapeutic delivery approaches in development for pediatric gliomas. CNS Oncol, 2013. 2(5): p. 427-35.

416. Roberts, W.G. and G.E. Palade, Increased microvascular permeability and endothelial fenestration induced by vascular endothelial growth factor. J Cell Sci, 1995. 108 ( Pt 6): p. 2369-79. 
417. Primeau, A.J., et al., The Distribution of the Anticancer Drug Doxorubicin in Relation to Blood Vessels in Solid Tumors. Clinical Cancer Research, 2005. 11(24): p. 8782-8788.

418. Hait, W.N. and T.W. Hambley, Targeted cancer therapeutics. Cancer Res, 2009. 69(4): p. 1263-7; discussion 1267.

419. Griffith, L.G. and M.A. Swartz, Capturing complex 3D tissue physiology in vitro. Nat Rev Mol Cell Biol, 2006. 7(3): p. 211-24.

420. Yamada, K.M. and E. Cukierman, Modeling tissue morphogenesis and cancer in 3D. Cell, 2007. 130(4): p. 601-10.

421. Lee, J., et al., In vitro toxicity testing of nanoparticles in 3D cell culture. Small, 2009. 5(10): p. 121321.

422. Polacheck, W.J., I.K. Zervantonakis, and R.D. Kamm, Tumor cell migration in complex microenvironments. Cell Mol Life Sci, 2013. 70(8): p. 1335-56.

423. Tchafa, A.M., et al., Three-dimensional cell culture model for measuring the effects of interstitial fluid flow on tumor cell invasion. J Vis Exp, 2012(65).

424. Godugu, C., et al., AlgiMatrix based 3D cell culture system as an in-vitro tumor model for anticancer studies. PLoS One, 2013. 8(1): p. e53708.

425. Tung, Y.C., et al., High-throughput 3D spheroid culture and drug testing using a 384 hanging drop array. Analyst, 2011. 136(3): p. 473-8.

426. Al-Farsi, A. and P.M. Ellis, Treatment paradigms for patients with metastatic non-small cell lung cancer, squamous lung cancer: first, second, and third-line. Front Oncol, 2014. 4: p. 157.

427. Leighl, N.B., Treatment paradigms for patients with metastatic non-small-cell lung cancer: first-, second-, and third-line. Curr Oncol, 2012. 19(Suppl 1): p. S52-8.

428. Leite, E.A., et al., Acute toxicity study of cisplatin loaded long-circulating and pH-sensitive liposomes administered in mice. J Biomed Nanotechnol, 2012. 8(2): p. 229-39.

429. Li, Y., et al., Paclitaxel-and/or cisplatin-induced ocular neurotoxicity: a case report and literature review. Onco Targets Ther, 2014. 7: p. 1361-6.

430. Banerjee, D., R. Harfouche, and S. Sengupta, Nanotechnology-mediated targeting of tumor angiogenesis. Vascular Cell, 2011. 3(1): p. 3. 
431. Ambudkar, S.V., et al., P-glycoprotein: from genomics to mechanism. Oncogene, 0000. 22(47): p. 7468-7485.

432. Chandolu, V. and C.R. Dass, Treatment of lung cancer using nanoparticle drug delivery systems. Curr Drug Discov Technol, 2013. 10(2): p. 170-6.

433. Babu, A., et al., Nanoparticle-Based Drug Delivery for Therapy of Lung Cancer: Progress and Challenges. Journal of Nanomaterials, 2013. 2013: p. 11.

434. Bertrand, N., et al., Cancer nanotechnology: the impact of passive and active targeting in the era of modern cancer biology. Adv Drug Deliv Rev, 2014. 66: p. 2-25.

435. Koziara, J.M., et al., In-vivo efficacy of novel paclitaxel nanoparticles in paclitaxel-resistant human colorectal tumors. J Control Release, 2006. 112(3): p. 312-9.

436. De Jong, W.H. and P.J. Borm, Drug delivery and nanoparticles:applications and hazards. Int J Nanomedicine, 2008. 3(2): p. 133-49.

437. Maione, P., et al., Combining targeted therapies and drugs with multiple targets in the treatment of NSCLC. Oncologist, 2006. 11(3): p. 274-84.

438. Eastman, A., The Mechanism of Action of Cisplatin: From Adducts to Apoptosis, in Cisplatin. 2006, Verlag Helvetica Chimica Acta. p. 111-134.

439. Kumar, N., Taxol-induced polymerization of purified tubulin. Mechanism of action. J Biol Chem, 1981. 256(20): p. 10435-41.

440. England, C.G., Miller, M.C., Kuttan, A., Trent, J.O., Frieboes, H.B., Release Kinetics of Paclitaxel and Cisplatin from Two and Three Layered Gold Nanoparticles. In Press, 2014.

441. Hoo, C., et al., A comparison of atomic force microscopy (AFM) and dynamic light scattering (DLS) methods to characterize nanoparticle size distributions. Journal of Nanoparticle Research, 2008. 10(1): p. 89-96.

442. Haycock, J., 3D Cell Culture: A Review of Current Approaches and Techniques, in 3D Cell Culture, J.W. Haycock, Editor. 2011, Humana Press. p. 1-15.

443. Allen, T.M., The use of glycolipids and hydrophilic polymers in avoiding rapid uptake of liposomes by the mononuclear phagocyte system. Advanced Drug Delivery Reviews, 1994. 13(3): p. 285309. 
444. Li, S.-D. and L. Huang, Nanoparticles evading the reticuloendothelial system: Role of the supported bilayer. Biochimica et Biophysica Acta (BBA) - Biomembranes, 2009. 1788(10): p. 2259-2266.

445. Schuster, B.G., et al., Production of antibodies against phosphocholine, phosphatidylcholine, sphingomyelin, and lipid A by injection of liposomes containing lipid A. J Immunol, 1979. 122(3): p. $900-5$.

446. Szebeni, J., C.R. Alving, and F.M. Muggia, Complement Activation by Cremophor EL as a Possible Contributor to Hypersensitivity to Paclitaxel: an In Vitro Study. Journal of the National Cancer Institute, 1998. 90(4): p. 300-306.

447. Bootz, A., et al., Comparison of scanning electron microscopy, dynamic light scattering and analytical ultracentrifugation for the sizing of poly(butyl cyanoacrylate) nanoparticles. European Journal of Pharmaceutics and Biopharmaceutics, 2004. 57(2): p. 369-375.

448. Xiao, K., et al., The effect of surface charge on in vivo biodistribution of PEG-oligocholic acid based micellar nanoparticles. Biomaterials, 2011. 32(13): p. 3435-46.

449. Frohlich, E., The role of surface charge in cellular uptake and cytotoxicity of medical nanoparticles. Int J Nanomedicine, 2012. 7: p. 5577-91.

450. Gandola, Y.B., et al., Mitogenic effects of phosphatidylcholine nanoparticles on MCF-7 breast cancer cells. Biomed Res Int, 2014. 2014: p. 687037.

451. Shin, C.S., et al., Development of an in vitro 3D tumor model to study therapeutic efficiency of an anticancer drug. Mol Pharm, 2013. 10(6): p. 2167-75.

452. Franko, A.J. and R.M. Sutherland, Oxygen diffusion distance and development of necrosis in multicell spheroids. Radiat Res, 1979. 79(3): p. 439-53. 


\section{APPENDIX}

Permission for "Enhanced penetration into 3D cell culture using two and three layered gold nanoparticles," was obtained from the International Journal of Nanomedicine.

International Journal of Nanomedicine by DOVE Medical Press. Reproduced with permission of DOVE Medical Press in the format Republish in a thesis/dissertation via Copyright Clearance

Center.

International Journal of Nanomedicine

Order detail ID: 65761127

ISSN: $\quad 1178-2013$

Publication Journal

Type:

Volume:

Issue:

Start page:

Publisher:

DOVE Medical Press

\section{Permission Status: $\checkmark$ Granted}

Permission type: Republish or display content

Type of use: Republish in a thesis/dissertation

Order License Id: 3458910048236

$\boxplus$ View details 


\section{CURRICULUM VITA}

\section{Christopher G. England}

Doctoral Candidate/Graduate Researcher University of Louisville, CTRB 227A

505 S. Hancock Street

Louisville, KY 40202, USA
Phone: (270) 872-3803

Email: christopher.england@louisville.edu

\section{Education}

$\begin{array}{llll}\text { University of Louisville } & \text { Ph.D. } & \text { 2011-2014 } & \begin{array}{l}\text { Pharmacology and Toxicology } \\ \text { Expected December 2014 }\end{array} \\ \text { University of Louisville } & \text { M.S. } & 2011-2013 & \text { Pharmacology and Toxicology } \\ \text { Bellarmine University } & \text { B.S. } & 2007-2011 & \text { Biochemistry \& Molecular Biology }\end{array}$

\section{$\underline{\text { Research }}$}

University of Wisconsin-Madison Postdoctoral Research Associate January 2015- current Project: Development of hybrid nanoparticles for the co-current treatment and imaging of cancer. Design of novel PET tracers for cancer patient management.

University of Louisville Graduate Research Assistant July 2011- December 2014 Project: Design, synthesis, and characterization of gold nanoparticles. Analyzed effects of drug loading and release kinetics with various surface modifications. Performed pharmacokinetic and pharmacodynamics analysis for determining tumor treatment response.

Bellarmine University Undergraduate Researcher August 2008- May 2011 Project: Identification of Penicillium and Aspergillus fungal species using novel PCR methods. Employed microarray analysis for determination of yeast present in sour mash for fermenting plants in Kentucky.

University of Louisville KBRIN Research Scholar $\quad$ May 2009- August 2009 Project: Examined the role of CD34 in pathogenesis of Microbotryum violaceum. The gene was spliced from the fungi, and transfected into bacteria using liposomes. Experience with PCR, transfection, and liposomes synthesis.

\section{$\underline{\text { Technical Skills }}$}

Cell Culture: Basic cell culture techniques, 3D cell culture, cytotoxicity assays

Genetic Analysis: PCR, Transfection

Protein Analysis: Western blot, Bradford assay 
Nanoparticle Design: Synthesis (metallic nanoparticles, nanoshells, liposomes, cancer-sensing probes with varying shapes), Nanoparticle functionalization (various methods), Nanoparticle characterization (various methods, see "Instruments"), Photothermal Therapy (laser ablation), Drug Loading and Release

Animal Model: Mouse Surgery, Mouse injection (I.V., I.P., Sub.Q.), Imaging (various methods, see "Instrumentation")

Instrumentation: Multispectral Optoacoustic Tomography (MSOT), Microscopy (SEM, STEM, AFM, Dark-Field), CytoViva Hyperspectral Imaging, Advanced Molecular Imager (Bioluminescence and Fluorescence), High Performance Liquid Chromotography (HPLC), Flow Cytometry, ZetaSizer (surface charge and dynamic light scattering), UV-Spectroscopy, Fourier Transform Infrared Spectroscopy (FTIR)

Programming: Some experience utilizing and writing code with $\mathrm{C}, \mathrm{C}++$, and MATLAB.

Miscellaneous: Lab Setup and Management

\section{Peer-Reviewed Publications}

1. Zeiderman MR, Egger ME, Kimbrough CW, England CG, Dupre TV, McMasters KM, and McNally LR. Targeting of BRAF resistant melanoma via EMMPRIN receptor. J Surg Res, 21 Feb. 2014.

2. England CG, Priest T, Zhang G, Sun X, Patel D, McNally LR, van Berkel V, Gobin AM, and Frieboes HB. Enhanced penetration into 3D cell culture using two- and three layered gold nanoparticles. Int J Nanomedicine, 01 Oct. 2013.

3. Lee J, Huang J, England CG, McNally LR, and Frieboes HB. Predictive modeling of in vivo response to gemcitabine in pancreatic cancer. PLOS Comp Biol, 19 Sep. 2013.

\section{Publications In Review}

1. England CG, Miller MC, Kuttan A, Trent JO, and Frieboes HB. Drug Release Kinetics of Paclitaxel and Cisplatin from Two- and Three Layered Gold Nanoparticles. 2014.

2. England CG, Huang J, James K, Zhang G, Gobin AM, McNally LR, and Frieboes HB. Detection of Phosphatidylcholine-Coated Nanoparticles in Orthotopic Pancreatic Adenocarcinoma using Hyperspectral Imaging. 2014.

3. England CG, $\mathrm{Ng} \mathrm{CH}$, van Berkel VT, and Frieboes HB. A Review of Pharmacological Treatment Options for Lung Cancer: Emphasis on Novel Nanotherapeutics and Associated Toxicity (2014).

4. England CG and Frieboes HB. Cytotoxicity of Cisplatin- and Paclitaxel- loaded Nanoparticles in NSCLC 3D Cell Cultures. 2014.

\section{Poster Abstracts}

1. England CG, Miller MC, Kuttan A, Trent JO, and Frieboes HB. Examining the Release of Paclitaxel and Cisplatin from Two- and Three Layered Gold Nanoparticles. James Graham Brown Cancer Center Retreat, Louisville, KY October 24, 2014.

2. Zeiderman M, Egger ME, England CG, Kimbrough CW, Dupre TV, McMasters KM, and McNally LR. EMPRINN expression provides a novel target for cell-specific targeting of metastatic melanoma. American Surgical Congress, February 4, 2014.

3. Kimbrough CW, England CG, Dupre TV, Huang J, Hudson SV, and McNally LR. Syndecan-1 probe selectively localizes to pancreatic adenocarcinoma. American Surgical Congress, February 4, 2014. 
4. England CG, Priest T, Zhang G, Sun X, Patel D, Gobin AM, and Frieboes HB. Enhanced diffusion of two- and three- layered nanoparticles in 3D cell cultures. James Graham Brown Cancer Center Retreat, Louisville, KY October 25, 2013.

5. Hudson SV, Kimbrough CW, Huang J, Dupre TV, England CG, and McNally LR. Optimal Imaging of Hypoxia in Pancreatic Adenocarcinoma. Research Louisville, Louisville, KY September 24, 2013.

6. England CG, Priest T, Zhang G, Sun X, Gobin AM, and Frieboes HB. Enhanced uptake of two- and three- layered nanoparticles in 3D cell cultures. Research Louisville, Louisville, KY September $24,2013$.

7. Pickelsimer DD, Dupre TV, Huang J, Kimbrough CW, Hudson SV, England CG, and McNally LR. Pancreatic Adenocarcinoma Detected with Targeted Liposomes. Research Louisville, Louisville, KY. September 24, 2013.

8. Zeiderman M, Egger M, Kimbrough CW, England CG, Dupre TV, McMasters K, and McNally LR. Novel Therapies for BRAF-Inhibitor Resistant Melanoma. Research Louisville, Louisville, KY, September 24, 2013.

9. Kimbrough CW, Dupre TV, England CG, Huang J, and McNally LR. Pancreatic Adenocarcinoma detected by Multispectral Optoacoustic Tomography using Syndecan-1 probe. Research Louisville, Louisville, KY, September 24, 2013.

10. Dupre TV, England CG, Huang J, and McNally LR. Detection of pancreatic adenocarcinoma in vivo with S100A9 liposomes. Research Louisville, Louisville, KY, September 24, 2013.

11. England CG, Friebeos HB, McNally LR. X-ray skeleton imaging in conjunction with bioluminescence imaging does not alter pancreatic tumors. World Molecular Imaging Congress, Savannah, GA, September 18-21, 2013.

12. England CG, Frieboes HB, McNally LR. Detection of orthotopic pancreatic tumor using multispectral optoacoustic tomography. World Molecular Imaging Congress, Savannah, GA, September 18-21, 2013.

13. Hudson C, England CG, Frieboes HB, McNally LR. Detection of tumors in mice using probes. World Molecular Imaging Congress, Savannah, GA, September 18-21, 2013.

14. England CG, Zhang G, Sun X, Gobin AM, Frieboes HB. Diffusion of two- and three- layered nanoparticles in 3D cell cultures. James Graham Brown Cancer Retreat, Louisville, KY, October 12, 2012.

15. England CG, Zhang G, Sun X, Gobin AM, and Frieboes HB. Optimization of Nanoparticle Penetration into Solid Tumors. Research Louisville, Louisville, KY, September 28, 2012.

\section{$\underline{\text { Scholarly Presentations }}$}

England CG, Huang J, James K, Zhang G, McNally LR, Gobin AM, and Frieboes HB. "Detection of Gold Nanoparticles in Orthotopic Pancreatic Adenocarcinomas using Hyperspectral Imaging,” 2014 Graduate Research Symposium, University of Louisville. Louisville, KY, USA. April 11, 2014.

England CG, Priest T, Zhang G, Sun X, Patel D, McNally LR, van Berkel V, Gobin AM, and Frieboes HB. "Two- and three- layer gold nanoparticles optimized for enhanced uptake in 3D cell cultures," Kentucky Academy of Science Annual Meeting, Morehead State University. Morehead, KY, USA. November 9, 2013.

England CG, Priest T, Zhang G, Sun X, Patel D, McNally LR, van Berkel V, Gobin AM, and Frieboes HB. "Enhancement of drug delivery using an experimental/computational approach," Department of Pharmacology and Toxicology, University of Louisville. Louisville, KY, USA. June 21, 2013.

England CG and Frieboes HB. "Nano-based therapeutics for the treatment of non-small cell lung cancer (NSCLC)." Department of Pharmacology and Toxicology, University of Louisville. Louisville, KY, USA. July 17, 2012 
England CG and Dobbins J. "Early detection of fungal infections in the clinic." Butler University Undergraduate Research Conference. Indianapolis, IN, USA. April 12, 2011

\section{Teaching Experience}

2014

2013-2014

2013

2012

\section{$\underline{\text { Service }}$}

2012-2014 Worked with high school students by engaging them in wet laboratory experience

2012-2014 Mentoring of M.S. students in laboratory projects and thesis dissertations
Dental Hygiene's Pharmacology Course- Instructor, University of Louisville

- Developed course materials and lectures covering topics such as drugs affecting the central nervous system (CNS), peripheral nervous system (PNS), and immune system.

- Facilitated lecture-based and discussion-driven teaching plans to promote active learning

- Developed critical thinking questions for discussion and exam purposes Undergraduate Basic Pharmacology Course-Instructor, University of Louisville

- Developed teaching plans and lectures on subjects including drug metabolism, drug elimination, antibiotics, and cardiovascular and renal pharmacology

- Provided additional learning opportunities outside of class time to ensure students were comfortable with material

Graduate Teaching Academy, University of Louisville

- This 12-month program covered topics such as: collaborative student work, classroom management, assessment techniques, active learning, productive discussions, teaching statements, learning-centered syllabi, micro-teaching, peer feedback, and synthesizing rubrics.

- Constructed personal philosophy of teaching statement along with professionally reviewed rubrics and syllabi for use in future courses

- Taught various subjects and received feedback to enhance teaching skills. High School Science Days-"Nanotechnology in Engineering," University of Louisville

- Facilitated group discussion on the topic of biomedical engineering with application in drug delivery to tumors

- Engaged high school students by providing visual demonstrations along with handson activities for active learning

\section{Awards, Honors, Fellowships and Scholarships}

2014

2013

2012

2011

2011

2009

2009

2007
Graduate Teaching Academy Certificate, University of Louisville

American Association of Pharmaceutical Scientists Mentor Program

First Place in Engineering Collaboration Award, Research Louisville

Interdisciplinary Program in the Biomedical Sciences Fellowship Recipient

First Year President for Department of Pharmacology and Toxicology

President of Biochemistry and Molecular Biology Bellarmine Organization

Kentucky Biomedical Research Infrastructure Network (KBRIN) Fellowship Recipient

Bellarmine University Honors Society 


\section{Professional Societies, Councils, and Committees}

2013-current

2013-current

2013-current

2012-current

2011-current

2007-2009

2008-2010

2007-2009
Ohio Valley Society of Toxicology

World Molecular Imaging Congress

American Society for Nanomedicine

American Association of Pharmaceutical Scientists

Kentucky Academy of Science

American Society for Biochemistry and Molecular Biology

American Chemical Society

American Association for the Advancement of Science 